\section{MOTICE}

PORTIONS OF THIS REPORT ARE ILLEGISL

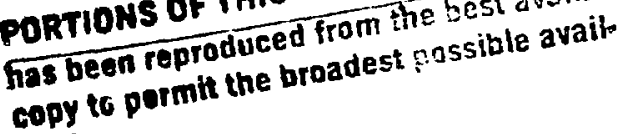

bility.

\title{
Elastic and Inelastic Scattering of Polarized Protons from Carbon-12 at 400, 600, and $700 \mathrm{MeV}$
}

$L A--10064-T$

DE\&4 013875

Kevin Wyndham Jones 


\section{DISCLA.IMER}

This report was prepared as an account of work sponsored by an agency of the United States Government. Neither the United States Government nor any agency thereof, nor any of their employees, makes any wasianty, express or implied, or assumes any legal liability or responsibility for the accuracy, completeness, or usefulness of any information, apparatus, product, or frocess disclosed, or represents that its use would not infringe privately owned rights. Reference ; : a in to any specific commercial product, process, or service by trade name, trademark, manufacturer, or otherwise does not necessarily constitute or imply its endorsement, recomnendation, or favoring by the United States Government or any agency thereof. The views and opinions of authors expressed herein do not necessarily state or reflect those of the United States Government or any agency thereof. 


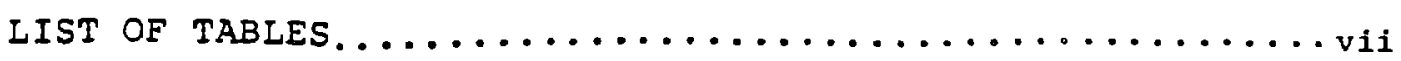

LIST OF FIGURES......................... viii

ABSTRACT ..............................

\section{CHAPTER}

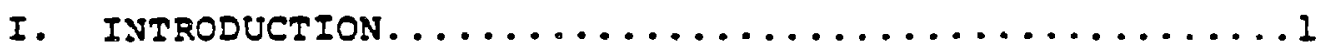

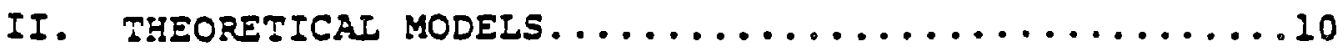

Optical Model Aralysis of Elastic Scattering...10

The Distorted Wave Impulse Approximation......13

An EEfective Nucleon-Nucleon Interaction for Intermediate Energy scattering............

III. EXPERIMENTAL METHOD.......................

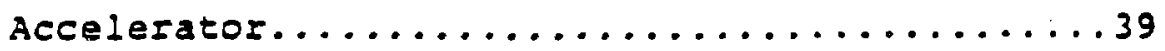

The High Resolution Spectrometer...........44

Iarsets............................46

Beam Monitors......................47

Focal Plane Detection System.............49

Electronics and Sata Acquisition............. Il

MBd Event Rejectisn......................

IV. DATA ANALYSIS AND REDLCEION..............60

Event Processing and Testing.............60

Peak Eitting......................64

Differential Cross Sections and Absolute

Nozmalization......................68

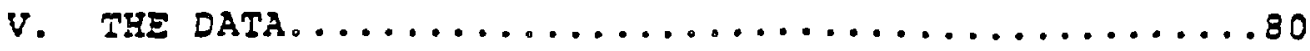

spectra............................80 
Differential Cross Sections and Analyzing

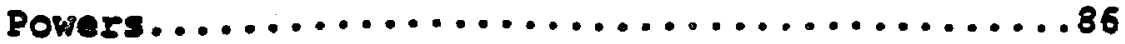

Isoscalar states of Natural parity.........86

Isovector States of Natural Parity.......115

Isoscalar states of Unnacural parity......121

Isovector States of Unnatural Parity......127

States of Indefinite Quantum Numbers for

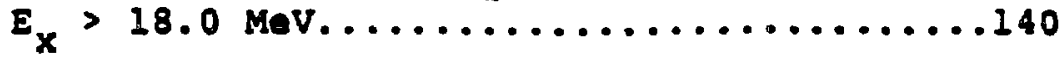

VI. THEORETICAL INTERPRETATIONS OF THE DATA. $\ldots \ldots \ldots 162$

Optical Model Calculations - Elastic

scattering.........................162

Distorted Wave Impulse Approximation

Calculations...........................

Isoscalar scates of Natural parity.........179

Isovector states of Natural Parity.......202

Isoscalar states of Unnatural Parity......215

Isovector States of Unnatural zarity.....224

States for $E_{x}>18.0 \mathrm{MeV} \ldots \ldots . \ldots \ldots \ldots . . . . .239$

vil. CONCLUSIONS..........................273

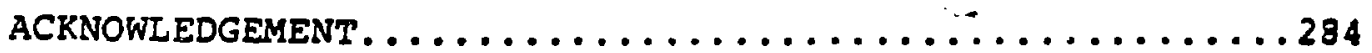

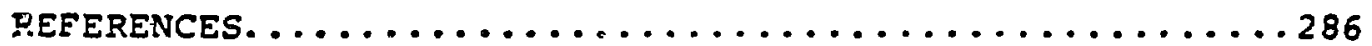

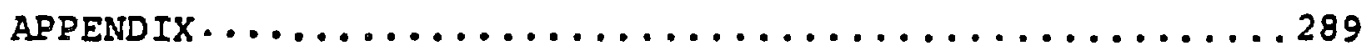




\section{IIST OF TABLES}

Page

TABLE I-1. - Energy Levels of ${ }^{12}$ c.................

TABIE IV-1. - Excitation Energies and Widths extracted from peak-fitting in the $18-21 \mathrm{MeV}$ region.......................67

TABLE IV-2. - Target Weight Ratios...............76

TABLE IV-3. - Relative and Absolute Cross Sections and Normalization Factors by Bin.........76

TABLE IV-4. - Absolute Normalization Factors..........79

TARLE VI-I. - Optical Model Potential Parameters......166

TABLE VI-2. . Reduced Matrix Elements.............176

TABIE VI-3. - Scale Eactors for DWIA Calculations.....272

TABLE VII-1. - High Excitation Energy Spin, Parity, and Isospin Assigrments (Tentative).......283

TABLE A-1. - Experimental Angular Distributions 398 Mev........................ 295

TABIE A-2. - Experimental Angular Distributions -

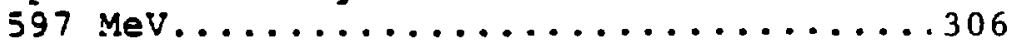

TA3E A-3. - Experimental Angular Distributions -

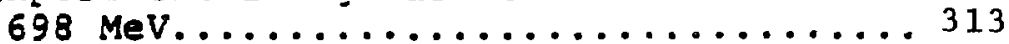




\section{LTST OF FIGURES}

Page

EIGURE I-1. - EnergY Levels of ${ }^{12} \mathrm{C} \ldots \ldots \ldots \ldots \ldots \ldots \ldots$

FIGURE I-2. - Shell Model orbitals used for ${ }^{12} \mathrm{C}$

Wavefunctions...................8

EIGURE II-1. - Strength of $N-N$ Interaction Components

$q=0.5 \mathrm{fm}-1 \ldots \ldots \ldots \ldots \ldots . \ldots \ldots$

FIGURE II-2. - Strength of N-N Interaction Components

$q=1.0 \mathrm{fm}-1 . \ldots \ldots \ldots . . \ldots . . . \ldots 26$

FIGURE II-3. - Strength of $N-N$ Interaction Components

$q=1.5 \mathrm{fm}^{-1} \ldots \ldots \ldots \ldots . . \ldots . . . \ldots 27$

EIGURE II-4. - Strength of $N-N$ Interaction Components

$q=2.0 \mathrm{fm}-1 . \ldots \ldots \ldots \ldots . \ldots . \ldots . \ldots . . . \ldots 28$

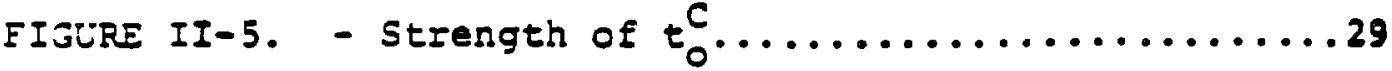

FIGURE II-6. - strength of $t_{0}^{c} \ldots \ldots \ldots \ldots \ldots \ldots \ldots \ldots \ldots . \ldots . \ldots . \ldots$

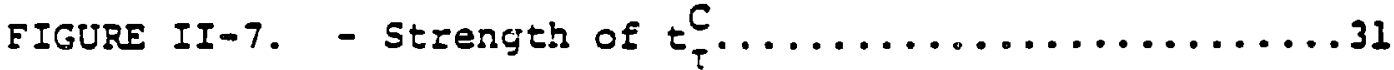

FIGURE II-8. - strength of $t_{\sigma \tau}^{c} \ldots \ldots \ldots \ldots \ldots \ldots \ldots \ldots \ldots$

EIGURE II-9. - Stzength of $t_{0}^{L S} \ldots \ldots \ldots \ldots \ldots \ldots \ldots \ldots \ldots . \ldots . \ldots 3$

FIGURE II-10. - strength of $t_{\tau}^{i S} \ldots \ldots \ldots \ldots \ldots \ldots \ldots$

FIGURE II-11. - Strength of $t_{0}^{T} \ldots \ldots \ldots \ldots \ldots \ldots \ldots \ldots$

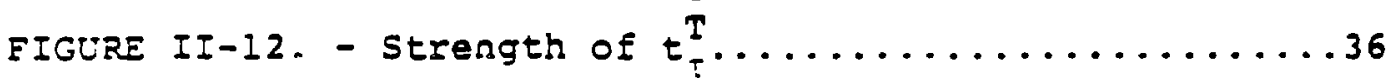

FIGURE III-1. - Experimental Areas Los Alamos Mesor

Physics Facility................42

FIGURE III-2. - Experimental Area ' $C$ '.............43

FIGURE III-3. - Section View High Resolution Spectro-

meter.......................45

EIGURE III-4. - HRS FOCal Plane Detector Array.........50

FIGURE III-5. - Data Acquisition System Schematic......53

FIGURE III-6. - HRS Fast TIIgger.................55

FIGURE III-7. - Raw $x$ POgition (1) gs. Missing Mass.....58 
FIGURE III-8. - Spectrum Showing MBD Cut............59

FIGURE IV-1. - Spectrometer Aagular Acceptance.......62

FIGURE IV-2. - Sample LOW Excitation Energy Missing

Mass Histogram.................65

FIGURE IV-3. - Low q High Excitation Energy Spectrum....69

FIGURE IV-4. - High G High Excitation Energy Spectrum...70

FIGURE $V-1 . \quad$ - Low Excitation Spectra................

FIGURE V-2. - Medium Exeitation Spectra............83

FIGURE V-3. - High Excitation Spectra..............

FIGURE $v-4 . \quad-{ }^{13} C$ Inelastic scattering spectrum.......88

FIGURE V-5. - Elastic Differential Cross Sections......90

FIGURE V-6. - Elastic Analyzing Powers............9l

FIGURE V-7. - Elastic - Comparative Observables......992

FIGURE V-8. $-E_{Y}=4.44 \mathrm{MeV} 2_{1}^{+} ; 0$ - Differential Cross

EIGLRE V-9. $\quad-\Xi_{X}=4.44 \mathrm{MeV} \mathrm{2}{ }_{1}^{+}: 0$ - Analyzing Powers....96

FIGLRE $V-10 . \quad-E_{\text {boservables... }}=4.44 \mathrm{MeV} 2^{+} ; 0$ - Comparative

FIGURE $v-11 . \quad-E_{X}=7.65 \mathrm{MeV} 0_{2}^{+} ; 0-$ Difserential Cross

FIGURE V-12. $-E_{X}=7.65 \mathrm{MeV} 0_{2}^{+}: 0$ - Analyzing Powers...100

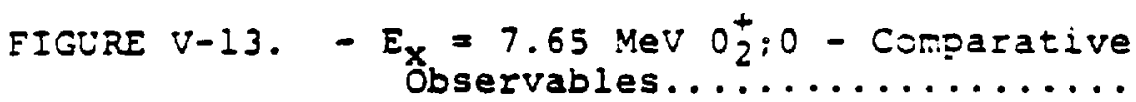

FIGURE V-14. $-E_{X}=9.64 \mathrm{MeV} 3 ; ; 0$ - Differential Cross Sections..........................

FIGURE V-15, $\quad E_{\mathbf{X}}=9.64 \mathrm{MeV} 3 \overline{1} ; 0$ - Analyzing Powers...105 FIGURE V-16. $-E_{X}=9.64 \mathrm{MeV} 3_{1}^{-}: 0$ - Comparative Observables....................106

FIGURE V-17. $-E_{X}=10.84 \mathrm{MeV} I_{1}^{-} ; 0$ - Differential Cross Sections...........................

FIGURE V-18. $-E_{X}=10.84 \mathrm{MeV} I_{I}^{-} ; 0$ - Analyzing Powers..110 


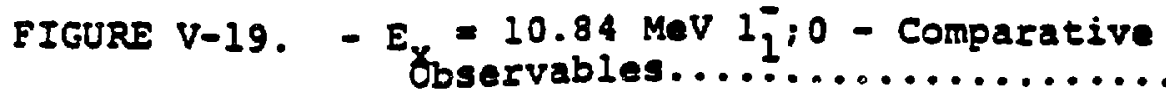
111

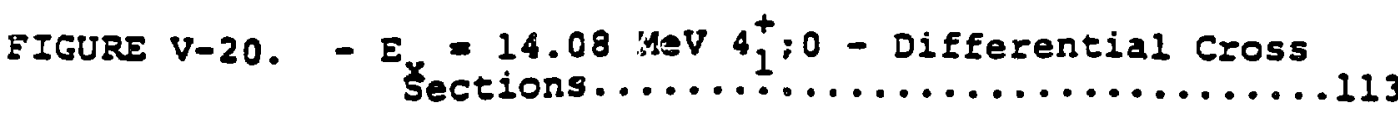

EIGURE V-21. $-E_{X}=14.09 \mathrm{MeV} 4_{1}^{+} ; 0$ - Analyzing Powers...114 FIGURE V-22. - E $=14.08 \mathrm{MeV} 4_{1}^{+} ; 0$ - Comparative

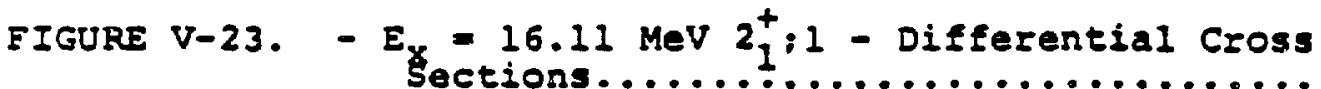
118

EIGURE V-24. $-E_{X}=16.11 \mathrm{MeV} 2_{1}^{+}: 1$ - Analyzing Powers...119 FIGURE V-25. - $E_{\gamma_{b s e}}=16.11 \mathrm{MeV} 2_{1}^{+} ; 1$ - Comparative

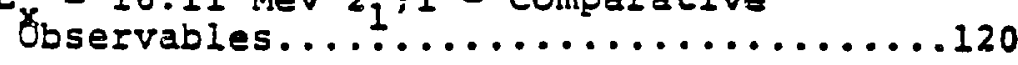

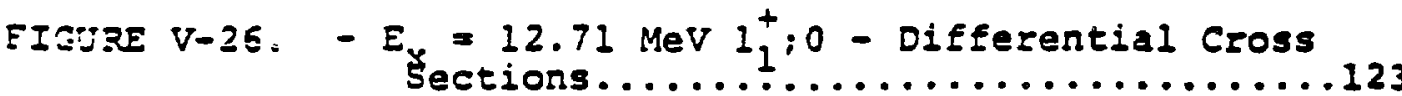

FIUURE V-27. $-E_{X}=12.71 \mathrm{MeV} 1_{I}^{+}: 0$ - Analyzing Powers...124 FIGURE V-28. - E $=12.71 \mathrm{MeV} 1_{1}^{+} ; 0$ - Comparative

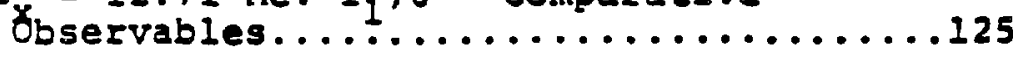

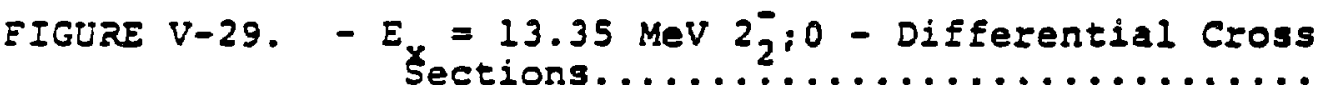

EIGURE V-30. $-E_{x}=13.35 \mathrm{MeV} \mathrm{2} ; ; 0$ - Analyzing Powers...129 FICURE V-31. - E $=13.35 \mathrm{MeV} 2{ }_{2} ; 0$ - Comparative

EIGURE V-32. - $E_{\text {Y }}=15.11 \mathrm{MeV} \mathrm{l}_{1}^{+} ; 1$ - DifEerential Cross Sections.............................

FIGURE V-33. - $E_{X}=15.11 \mathrm{MeV} 1_{1}^{+} i l$ - Analyzing Powers...233

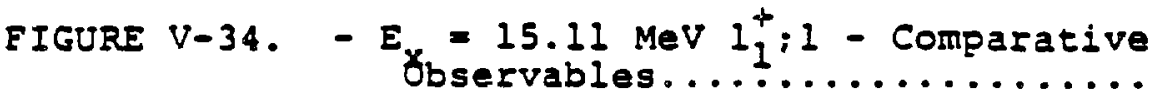
.134

FIGURE V-35. - $E_{\text {Ye }}=16.58 \mathrm{MeV} 2_{j}^{-} i l$ - Differential Cross

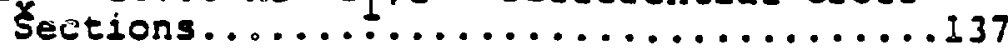

EIGURE V-36. $-E_{X}=16.58 \mathrm{MeV} 2_{1}^{-}: 1$ - Analyzing Powers...138 FIGURE V-37. - E Xbservables $=16.58 \mathrm{MeV} 2-11$ - Comparative

FIGURE V-38. - Ex $=18.30 \mathrm{MeV}$ - Differential Cross setctions........................... 
FIGURE V-39. $-E_{X}=18.30 \mathrm{MeV}$ - Aralyzing Powers.......142 FIGURE V-40. - $E_{X}=18.30 \mathrm{MeV}$ - Comparative Observables.143

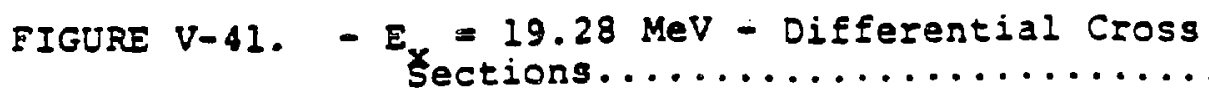
146

EIGURE V-42. - $E_{X}=19.28 \mathrm{MeV}$ - Analyzing Powers........147 EIGURE $V-43 .-E_{X}=19.28 \mathrm{MeV}$ - Comparative Observables.148 FIGURE V-44. - $E_{X}=19.40 \mathrm{MeV}$ - Differential Cross

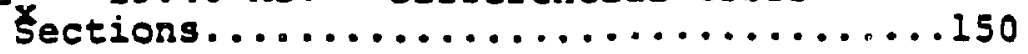

EIGURE V-45. - $E_{X}=19.40 \mathrm{MeV}$ - Analyzing Powers........ I5I FIGLRE V-46. - $E_{X}=19.40 \mathrm{MeV}$ - Comparative Observables.152 FIGURE V-47. - $E_{\text {. }}=19.65 \mathrm{MeV}$ - Differential Cross Šections...........................

FIGURE V-48. $-E_{X}=19.65 \mathrm{MeV}$ - Analyzing Powers........155 EIGURE V-49. $-E_{X}=19.65 \mathrm{MeV}$ - Comparative Observables.156 FIGURE $V-50$. $-E_{X}=20.60 \mathrm{MeV}-$ Differential Cross

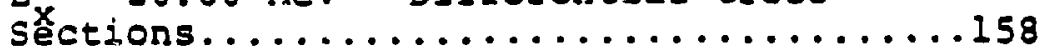

FIGURE V-51. - $E_{X}=20.60 \mathrm{MeV}$ - Analyzing Powers........159 EIGLRE V-52. - $E_{X}=20.60 \mathrm{MeV}$ - Comparative Observables.160 FIGURE VI-1. - Elastic Scattering Differential Cross Section Optical Fit - 393 MeV.........164

FIGURE VI-2. - Elastic Scattering Analyzing Power Optical Fit - $398 \mathrm{MeV} . \ldots \ldots \ldots \ldots \ldots \ldots 65$

FIGURE VI-3. - Elastic scattering Differential Cross Section Optical Fit - 597 Mev..........169

FIGURE VI-4, - Elastic Scattering Analyzing Power Optical Fit - 597 Mer.............170

FIGURE VI-5. - Elastic Scattering DifEerential Cross Section Optical Fit - 698 Mev.........171

FIGURE VI-6. - Elastic Scattering Analyzing power Optical Fit - 698 Mev.............172

FIGURE VI-7. - Key for Curves in Figures VI-8 to VI-56..178 
FIGURE VI-8. $-E_{\text {I }}=4.44 \mathrm{MeV} 2+; 0-$ Differential Cross Section Calculations................180

FIGURE VI-9. $-E_{\mathcal{L}}=4.44 \mathrm{MeV} 2_{l}^{+} ; 0$ - Analyzing Power Calculations........................

FIGURE VI-10. - $E_{\mathrm{X}}=4.44 \mathrm{MeV} 2_{1}^{+} ; 0$ - Comparative Ealculations for observables.........182

FIGURE VI-11. - $E_{\text {N }}=4.44 \mathrm{MeV} \mathrm{2}+0$ - Renormalized bifferentiel choss sections..........183

FIGURE VI-12. - E $=9.64 \mathrm{MeV} 3 ; ; 0$ - Differential Cross Section Calculdtions..................189

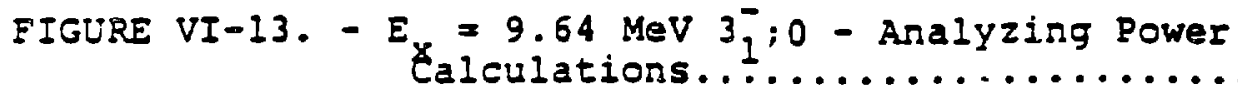

FIGURE VI-14. - E Ealculations for observables...

EIGURE VI-15. - $E_{x}=9.64 \mathrm{MeV} 3 ; ; 0$ - Renormalized Differential Cross sections............192

FIGURE VI-16. - $E_{\mathrm{X}}=10.84 \mathrm{MeV} I^{-} ; 0$ - DifEerential Cross Section Calculations................196

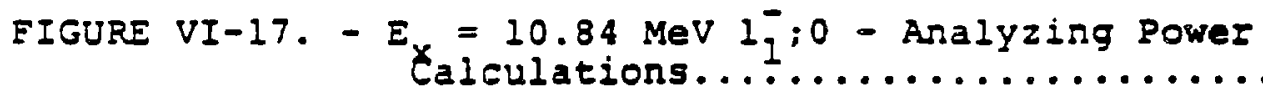

FIGURE VI-18. - $E_{\text {Ealculations fof }}=10.84 \mathrm{MeV}$ I $^{-}$Observables...

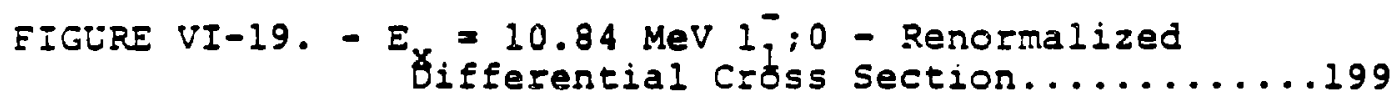

FIGLRE VI-20. $-E_{\text {Tet }}=16.11 \mathrm{MeV} 2_{j}^{+} 1$ - Differential Cross Section Calculations (Cnquenched).....205

EIGURE VI-21. - $E_{X}=16.11 \mathrm{MeV} 2_{l}^{+} ; 1$ - Analyzing Power Ealculations (uñquenched)...........206

FIGURE VI-22. - E $=16.11 \mathrm{MeV} 2_{1}^{+} ; 1$ - Comparative EIGURE VI-23. - $E_{X}=16.11 \mathrm{MeV} 2_{l}^{+} I$ - DifEerential Cross Section Calculations (Quenched).......208 FIGURE VI-24. - E Calculations $_{2}=16.11 \mathrm{MeV} 2^{+}$- Analyzing Power EIGURE VI-25. - E $=16.11 \mathrm{MeV} 2_{1}^{+}: 1$ - Comparative talculations for Observables (Quenched). 210 


\section{FIGURE VI-26. - $E_{\text {T }}=16.11 \mathrm{MeV} 2_{1}^{+} ; 1$ - Renormalzed}

bifferential Cróss Sections (Both)....211

FIGURE VI-27. $-E_{1}=12.71 \mathrm{MeV} 2_{1}^{+}: 0-$ Differential Cross

Section Calculatiops..............217

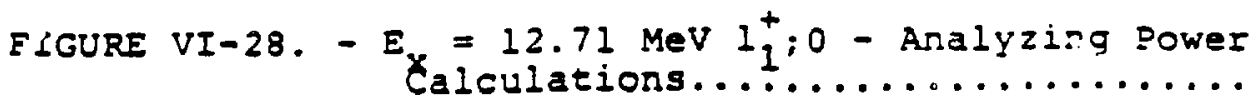

EIGURE VI-29. - E $=12.7 I \mathrm{MeV} \mathrm{I}_{1}^{+} ; 0$ - Comparative Calculations for Observables..........219

EIGURE VI-30. - $E_{\text {f }}=15.11 \mathrm{MeV} 1_{1}^{+} 11$ - Differential Cross Section Calculations..............226

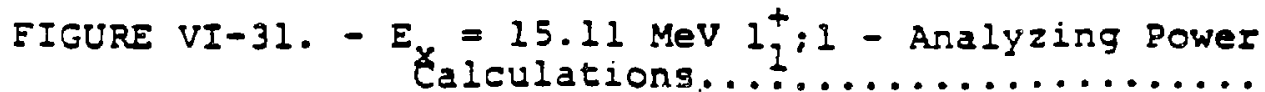

EIGURE VI-32. - $E_{\text {Calculations fol }}=15.11 \mathrm{MeV} 1^{+} ; 1$ - Comparative

FIGURE VI-33. - $E_{X}=16.58 \mathrm{MeV} 2 \bar{i}$ - Differential Cross Section Calculations...............234

FIGURE VI-34. - $E_{X}=16.58 \mathrm{MeV} 2-i I$ - Analyzing Power Ealculations.....................235

FIGURE VI-35. - $E_{X}=16.58 \mathrm{MeV} 2 \overline{1} ; 1$ - Compazative Calculations fol observables.......236

FIGURE VI-36. - $E_{\text {J }}=16.58 \mathrm{MeV} 2 j i l-$ Renormalized bifferential cróss sections.........237

FIGLRE VI-37. - $E_{\mathrm{X}}=18.30 \mathrm{MeV} 2{ }_{2} ; 0$ - DiEferential Cross Section Calculations.....................

FIGURE VI-38. - E $=18.30 \mathrm{MeV} 2 \overline{2} ; 0$ - Analyzing Power talculations......................242

FIGURE VI-39. $-E_{X}=18.30 \mathrm{MeV} 2 ; ; 0-$ Compaza:ive Zalculations Eor' Observajles........243

FIGURE VI-40. - $E_{\text {Y }}=19.28 \mathrm{MeV} 4 \bar{j} ; 0-$ DisEerential Cross Section Calculations...............249

FIGURE VI-41. $-E_{X}=19.28 \mathrm{MeV} 4{ }_{I} ; 0$ - Analyzing Power

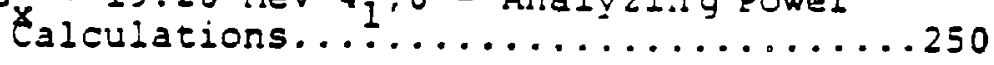

FIGLRE VI-42. $-E_{\text {Xalculations }}=19.28 \mathrm{MeV} 4_{1}^{-}: 0$ - Comparative talculations fof́ Observabies.........251

FIGURE VI-43. - $E_{X}=19.65 \mathrm{MeV} 4 j ; 1-D i s$ - Dential Cross .252 
EIGURE VI-44. $-E_{X}=19.65$ MeV $4 I: I-$ Analyzing Power

FIGURE VI-45. - $E_{Y}=19.65 \mathrm{MeV} 44_{1}^{-} ;$- Comparative

Calculations fot Observables.........254

FIGURE VI-46. - $E_{X}=19.28 \mathrm{MeV} \mathbf{1}_{2}^{-} ; 1$ - Differential Cross

Section Calculations...............255

FIGURE VI-47. $-E_{\text {X }}=19.28 \mathrm{MeV} 1_{2}^{-}: 1$ - Analyzing Power

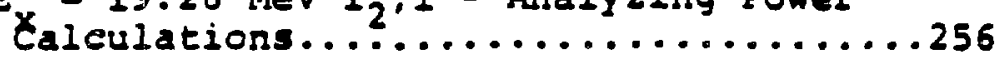

FIGURE VI-48. $-\Sigma_{\text {I }}=19.40 \mathrm{MeV} 2{ }_{2}: 1$ - Differential Cross

Section Calculations..............259

EIGURE VI-49. - $E_{X}=19.40 \mathrm{MeV} 2 ; ; I$ - Analyzing Power

Calculations....................260

FIGURE VI-5C. - $E_{X}=19.40 \mathrm{MeV} 2 \overline{2} ; 1$ - Comparative Calculations for Observables.........26I

FIGURE VI-51. - $E_{X}=19.40 \mathrm{MeV} \mathrm{l}_{\mathrm{I}}^{-} \mathrm{I}_{\text {- Differential Cross }}$ Section Calculations................262

FIGURE VI-52. - E $=19.40 \mathrm{MeV} 1_{j}: 1$ - Analyzing Power .263

EIGURE VI-53. - $E_{X}=19.40 \mathrm{MeV} 1_{j}^{-} i$ - Comparative Caiculations fot observabies.........264

FIGURE VI-54. - $E_{\text {Fection }}=20.60 \mathrm{MeV} 3-i l-D i f f e r e n t i a l$ Cross Section Calculations...............268

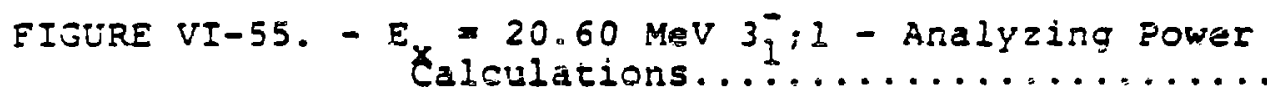
.269

FIGURE VI-56. - Exalculations fol Observabies... 270 


\section{ABSTRACT OE THE THESIS \\ Elastic and Inelastic Scattering of \\ Polarized Protons from Carbon- 12 \\ at 400,600 , and $700 \mathrm{MeV}$ \\ bY KEVIN WINDHAM JONES}

Thesis Director: Professor Charles Glashausser

Good resolution cross section and analyzing power $\left(\vec{p}, p^{\prime}\right)$ data for many states in ${ }^{12} C$ up to an excitation ener૬Y of $21 \mathrm{MeV}$ and spanning a momentum transfer range of 0.3 to $2.1 \mathrm{fm}^{-1}$ were obtained using the High Resolution Spectzometer at the Clinton P. Anderson Meson Physics Facility at incident beam energies of 398,597 , and $698 \mathrm{MeV}$. Optical model potentials were obtained from the elastic scattering data. Inelastic data were analyzed in the bistorted wave Impulse Approximation using the Love-Franey effective nucleon-nucleon interaction. The energy dependent isoscalar natural parity cross sections were uncerestimated, while phase difficulties were encountered in fitting analyzing powers. The energy independent isovector ratural parity cross sections were reasonably reproduced, but analyzing powers were not, the calculations yielding hositive trends whereas the data are of opposite sign. The energy independent isoscalar and isovector unnatural parity cross sections were quite well reproduced up to moderate momentum transfers, and striking successes were observed for some analyzing power data. An example is the success achileved in 
reprodueing the strong negative low momentum transfer dependence of the analyzing power for the $\varepsilon_{x}=12.71 \mathrm{MeV} 1^{+} ; 0$ state at $398 \mathrm{MeV}$. The strengths of the dominant terms in the 425 Mev force were found to be qualitatively and quantitatively well determined. A systematic lack of strength of some 20 308 in all dominant components of the $650 \mathrm{MeV}$ force was observed. Nevertheless, the Love-Franey parameterization of the nucleon-nucleon interaction is shown to provide a useful description of inelastic intermediate energy proton scattering from the ${ }^{12} \mathrm{C}$ nucleus, although some refinement is needed. Knock-on exchange contributions were found to be essential, generally interfering destructively with direct amplitudes, and optical model distortions influenced both cross sections and analyzing powers of some states. Systematics of energy dependence together with the results of the DWIA calculations permitted the assignment of spin, parity and isospin quantum numbers to states in the 18-21 MeV excitation region. 


\section{INTRODUCTION}

In recent years there has been much interest in the scattering of a variety of intermediate energy probes from different nuciei. Particies such as eiectrons, protons, neutrons and pions provide a rici and comprehensive testing ground for nuciear effective interactions, nuciear structure, and nuciear reaction theories.

Electrons, which interact with the nucleus through the weli-known electromagnetic and weak interactions, provide information on charge density distributions in nuelear matter. In addition, harmonic osciicator parameters used in describing the radial distribution of different nuciear states may be well determined by fitting the measured electror. scattering form factors for the states in question. such weif-determined information can then be used to fix parameters in the anaiysis of the scattering of more compiex probes, such as nucieons. Furthermore, ineiastic $180^{\circ}$ eiectron scattering is sensitive oniy to the transverse eiectromagnetic form factor, and hence can be used to selectiveiy excite nucieas states requiring a transfer of one unit of spin and isospin. In this way electron scattering tests the distribution of magnetism in nuclel.

Pions, which are intrinsie isovector partlcies, can, through charge exchange reactions and lnelastlc scattering, provide differing sensitivites to neutron and proton distri- 
butions within the nucleus. Such experiments yield important information concerning the dominant isospin components in the structure of some nuclear states, partlcularly isospin mixing between states of the same spin and parity.

Protons are an especialiy desirabie but complex proble of the nucleus. The proton can excite a wide variety of states in a given nueleus which may or may not be acceso sible by other modes of excltation. For example, eiastic scattering measurements, which leave the target nucleus in a residuai state without excitation, provide determinations of neutron and matter distributions which cannot be made with other probes. A study of differential cross sections and anaiyaing powers for ineiastic transitions, in which the residual nucieus is left in a weil-defined state of finite excitation energy, provides a means for extracting trans!tion densities, particularly neutron transition densities, for transtions from the ground state to the excited state. Both elastic and inelastic scattering data test the validity of microscopic modeis of reaction theory such as Glauber ${ }^{1}$ or KMT (Kerman, Memanus and Thaler) ${ }^{2)}$ theories. It is aiso possibie to test available parameterizations of the free nucieon-nucleon interaction, such as that proposed by love and Franey ${ }^{3)}$. Contributions of muiti-step processes to the excitation of ineiastic states can be investigated through coupled-channels calcuiations.

Information gained from the three probes mentioned 
provides interlocking pieces in the puzzles of nuclear structure and nuclear reaction theory. Each set of measurements serves as a guide to the interpretation of the physicai processes invoived in nuclear excitations, and the complementarity of the information so gained aijows reilatie deductions to be made concerning nuciear structure and reaction theory.

The ${ }^{12} \mathrm{C}$ nucleus, Iike all even-even nuclel, has a ground stare spin and parity of $\theta^{+}$, wirich provides useful simpileications in computations. This nucieus also exhibits a richness in discrete states of particle-hole character with reasonabie excitation energies ${ }^{4}$. For excitatiori energies bejow $18 \mathrm{MeV}$ a wide variety of states of small width, of order $100 \mathrm{keV}$, are easily resoived from broader states of width greater than $1 \mathrm{MeV}$ which form an underiying background. In addition to isoscaiar ( $\Delta T=\emptyset$ ) states of 'naturai' parity $\left(\pi=(-1)^{\mathrm{J}}\right)$, there exist both isoscajar and isovector $\left(\lfloor T=1)\right.$ states of 'unnatural' parity $\left(\pi=(-1)^{J+1}\right)$; for exampie the $\left(J^{\pi} ; T\right)=1^{+} ; \theta$ states at $E_{x}=12.71 \mathrm{MeV}$ and the $1^{+} ; 1$ state at $E_{x}=15.11 \mathrm{MeV}$. Aiso, an isovector state of 'naturai' parity $\left(2^{+} ; 1\right)$ is readily seen at $E_{x}=16.11 \mathrm{MeV}$. A level alagram of this nucleus is shown in Figure $I-1$, and a tabuiation of known states together with their intrinsic widths is given in Table I-1. The wide variety of states requiring different modes of excltation provides a considerable degree of sensitivity to the various components of the 
Energy Ievels of ${ }^{12} \mathrm{C}$

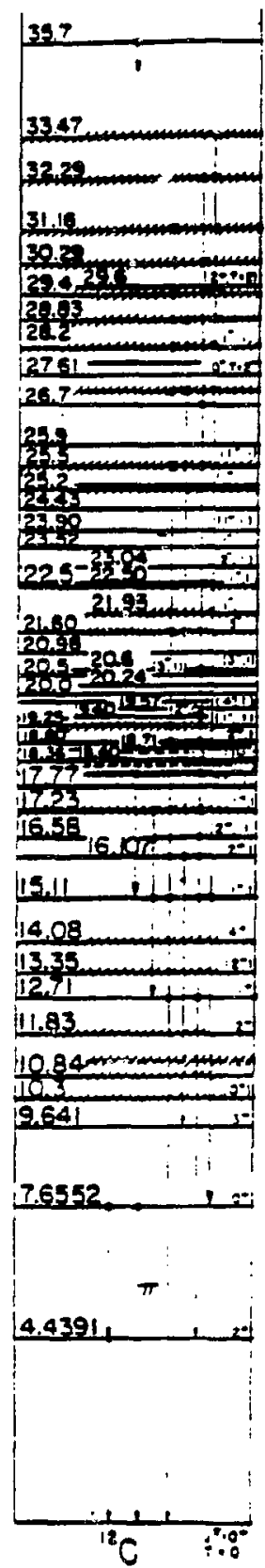

Eigure I-1. 
Energy Levels of ${ }^{12} \mathrm{C}$

\begin{tabular}{|c|c|c|}
\hline$E_{x}(\mathrm{MeV})$ & $\mathrm{J}^{\top} ; \mathrm{T}$ & $\Gamma(\mathrm{keV})^{\div}$ \\
\hline 0.00 & $0^{+} ; 0$ & \\
\hline 4.44 & $2^{+} ; 0$ & $10.8=0.6 \mathrm{mev}$ \\
\hline 7.65 & $0^{+} ; 0$ & $8.7 \div 2.7 \mathrm{ev}$ \\
\hline 9.64 & $3^{-}: 0$ & $34 \pm 5 \mathrm{keV}$ \\
\hline 10.3 & $\left(0^{+}\right): 0$ & $3000 \pm 700$ \\
\hline 10.34 & $1^{-} ; 0$ & $315=25$ \\
\hline 11.83 & $2^{-} ; 0$ & $260 \pm 25$ \\
\hline 12.71 & $I^{+} ; 0$ & $14.6 \pm 2.6 \mathrm{eV}$ \\
\hline 13.35 & $\left(2^{-}\right) ; 0$ & $375 \pm 40 \mathrm{kev}$ \\
\hline 14.08 & $4^{+t} ; 0$ & $258=15$ \\
\hline 15.11 & $1^{+}: 1$ & $42=7 \mathrm{eV}$ \\
\hline $16 . \therefore 1$ & $2^{+} ; 1$ & $6.5 \pm 0.6 \mathrm{keV}$ \\
\hline 16.58 & $2^{-} ; 1$ & 300 \\
\hline 17.23 & $1-11$ & 1150 \\
\hline 17.78 & $0^{+}: 1$ & $80 \pm 20$ \\
\hline 13.13 & $\left(2^{+} ; 0\right)$ & $600=100$ \\
\hline$(18.27)$ & $\left(4^{-} ; 0\right)$ & $275=40$ \\
\hline 18.36 & $\left(3^{-} ; 1\right)$ & $210=40$ \\
\hline 18.40 & $0^{-} ;(1)$ & 43 \\
\hline$(.38 .6)$ & $\left(3^{-}\right): ?$ & 300 \\
\hline 18.71 & $? ;(1)$ & 100 \\
\hline 18.80 & $2^{+} ; 1$ & $80=30$ \\
\hline 19.25 & $\left(1^{-} ; 1\right)$ & 1100 \\
\hline 19.40 & $\left(2^{+} ; 0\right)$ & 45 \\
\hline 19.57 & $\left(4^{-} ; 1\right)$ & $400=60$ \\
\hline 20.0 & $\left(2^{+}\right) ; ?$ & 90 \\
\hline 20.24 & & 170 \\
\hline 20.5 & $\left(3^{+} ; 1\right)$ & 250 \\
\hline 20.6 & $\left(3^{-} ; 1\right)$ & $200=40$ \\
\hline
\end{tabular}

Table I-1.

tinless otherwise noted 
nucleon-nucleus effective interaction which excites them.

We present in this work data for, and diseusston of, eiastic and inelastic scattering of polarized protons from ${ }^{12} \mathrm{C}$ at incident proton beam energies of 398, 597, and 698 MeV. The range of excitation energy spanned in the residual ${ }^{12} \mathrm{C}$ nucieus is from 0.0 to $21.0 \mathrm{MeV}$. We discuss the elastic scattering data briefly within the framework of the staidard nuciear optical model. The data for a wide range of ineiastic states is discussed within the framework of the loveFraney nucieon-nucieon interaction and the distorted wave Impulse Approximation (DWIA) ${ }^{2}$ ).

Data at incident bean energies of 120, 155, 180, 200, 402, 600, and $800 \mathrm{MeV}$ have recently been acquired5-16), using both poiarized and unpolarized beams. The data at 402 and $600 \mathrm{MeV}$ in particular were taken with unpoiarized beam and yieided only differential cross sections for a few states. Our extension of the measurements to beam energies between 200 and 800 MeV with poiarized beam thus provide an aimost complete set of differential cross section and anaiyzing power data spanning an incident beam energy range of some $800 \mathrm{MeV}$. The analyzing power measurements provide spin-dependent data which exhibit particular sensitivity to spin-dependent terms in the nucieon-nucleon effective interaction. Such sensitivity may impose further constraints on interactions used in th.oretical descriptions of the data. 
The benefits of obtaining data over such a wide range of incident beam energy are as foliows. First, the energy dependence of the data will provide usefui systematics for states of differing modes of excitation. Second, the energy dependence of effective nucieon-nucieon interactions, especiaily the Love-Franey force, can be studied in some detail. Third, the effects of such phenomena as pauil biocking and knock-on exchange can be investigated as a function of incident beam energy. These effects are of importance in the practical computation of observabies in the DWIA, and may aiso provide information on the applicabiitty of the impulse approximation itself.

From a nuciear structure point of view, several sets of sheil-modei wavefunctions for lp-sheii nuciei, of which ${ }^{12} \mathrm{C}$ is one, are avaiiable. Some such p-sheii wavefunctions. caicuiated by cohen and kurath ${ }^{17}$ ) have received extensive examination. Miliener ${ }^{18}$ ) has recentiy derived an extensive set of wavefunctions for negative parity states in ${ }^{12} c$. These wavefunctions inciude components invoiving the id and 2s-sheijs. Flgure I-2 shows a schematic representation of the sheii structure of $12 \mathrm{C}$ appicicabie to such wavefunctions. The data obtained in this work provide a usefui test of the validity of these wavefunctions.

Previous studies of the 'unnatural' parity states have been suggestive of valious phenomena which were deemed to warrant further study in thls work. The $1^{+} ; 0$ state was 
Shell Model Orbitals used for ${ }^{12} \mathrm{C}$ wavefunctions
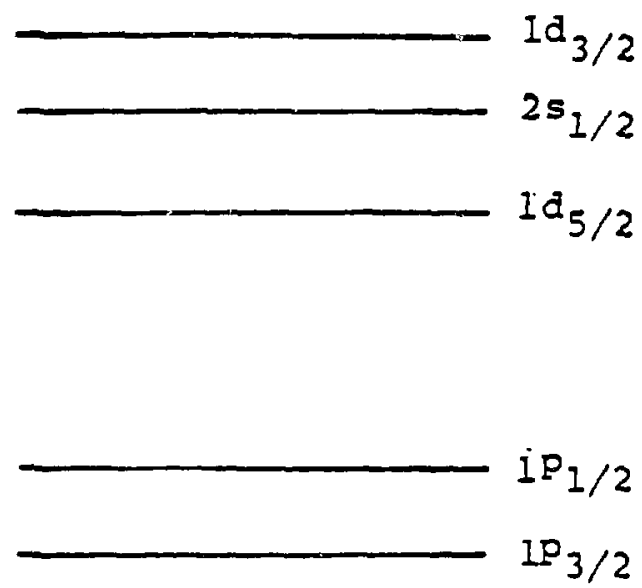

$\operatorname{ls}_{1 / 2}$

Figure I-2. 
observed to have a negative anaiyzing power at iow momentum transfer for an incident beam energy of $900 \mathrm{MeV}^{18}$ ). Such a property has been suggested as a signature for states excited by $i S=1, \quad I T=0$ mechanisms. We investigate this assertion in this work. Furthermore, several authors 20-23) proposed the $1^{+} ; 1$ state as a suitable candlate for the observation of precritical phenomena related to plon condensation. Current data available at lower energies $8,9,12$ ) and at $800 \mathrm{MeV}^{15}$ are not supportive of this assertion; the data obtalned in this work also show no discernibie ev1dence.

The excitation energy region from 18 to 21 MeV has aiso been the subject of considerabie recent investigation through the use of ineiastic scattering of eiectrons ${ }^{24}$, , protons ${ }^{91}$, and pions 25,26$)$, as weij as nucieon transfer reactions ${ }^{28}$ ). This region is difficuit to analyze because the stares existing there are broad and overiapping. The data obtained in our work, taken with other availabie information and the resuits of DWIA caicuiations, provide information on spin, parity and isospin assignments in this cegion. 


\section{THEORETICAL MODELS}

\section{A) Optical Medel Analysis of Elastic Scattering}

We treat the optical model analysis of the elastic scattering in a phenomenological way. In this approach the complex reaction of an incident projectile with a many-body nuclear system is reduced to the simpler problem of the interaction of the incldent projectile and the target nucleus as a whole in the presence of an average potential. The absorption of particles due to the existence of non-elastic chennels is accounted for by the inciusion of imaginary components in the average potentials. In addition, the scattering of a proton from a ${ }^{12} \mathrm{C}$ nucieus is a case of the scattering of a spin $\frac{1}{2}$ projectiie from a spin 0 target. Spin-orbit effects are therefore important and a complex spin-orbit potential of the Thomas form is inciuded in the description of the interaction.

The strategy of a phenomenoiogical optical anaiysis is as foijows. A guess is made at a potential, and the Schrödinger equation for the scattering process is solved in a partial wave expansion. The physical observabies are then computed from this soiution and compared with data. The potentiai parameters are then varied to optimize the quaiity of the fit of the calculated observables to the data.

Adopting a standard partial wave expansion for the waye function of the scattered spin $\frac{1}{2}$ particie, we may wrdte 
this wavefunction as

$$
y=\sum_{2} i^{2}[4 \pi(22+1)] \cdot 5(2 \varnothing s u \mid j m)(2 \lambda s v \mid j m) E_{2 j}(k r) Y_{2}^{\lambda}\left(\theta, \$ j k_{s} e^{i \sigma} \ell\right.
$$

where $(2 \varnothing \mathrm{s} \perp \mathrm{jm})$ is a Clebsch-Gordan coefficient coupling angulat nomenta $l$ and $s$ to a state of definite $j$ and projection $m$. The $f_{2 j}$ is a partial scattering amplitude from state $\&$ to state $j, x_{s}^{\mu}$ is the spin wave function, $Y_{2}^{\dot{\alpha}}(\vartheta, \downarrow)$ is a spherical harmonic and $e^{i \sigma l}$ is the coulomb phase Eactor.

$$
\begin{aligned}
& \text { We snow define the radial functions } u_{2 j}(k r) \text { such that } \\
& \qquad u_{2 j}(k r)=k_{i}\left[f_{\ell j}(k r)\right] \\
& I I-2
\end{aligned}
$$

This transformation results in a simplification of the radial schrodinger equation. The spin-orbit component requires the espectation values of the operator $\vec{L} \cdot \vec{s}$, which are given by

$$
\langle\vec{L} \cdot \vec{S}\rangle=\frac{1}{2}[j(j+1)-\chi(2+1)-s(s+1)] \quad I I-3
$$

Sirce the projectile has hals integral spin, $j=i=\frac{1}{2}$ and the eigen:ailues of $\langle\vec{I} \cdot \vec{S}\rangle$ are simply $i$ and $-(\vec{Z}+I)$ respectively. The radial part of the Schrodinger equation may tinen be written as

$$
\begin{aligned}
{\left[\frac{d^{2}}{d r^{2}}+k^{2}-\right.} & \frac{2(2+1)}{r^{2}}+v f(r)+i w g(r)-v_{c}(r) \\
& \left.+\left(v_{s o}+i w_{s 0}\right) h(r) i-(i+1) i\right] u_{i j}(k=)=0
\end{aligned}
$$

The $u_{2 j}(k r)$ are solutions corresponding to $j=2=\frac{1}{2}$. The $u_{\ell j}$ functions are linear combinations of the irregular coulomb functions $F$ and $G$ because of the presence of the coulomb 
potential required to describe proton scattering. The shape functions are of the woods-Saxon form appropriate for a spherical nucleus and are given by

$$
\begin{aligned}
& f(r)=\left[1+\exp \left(\frac{r-R}{a}\right)\right]^{-1} \\
& W g(r)=\left[W-4 W_{s} \frac{d}{d x^{\prime}}\right]\left(1+e^{X^{\prime}}\right)^{-1} \\
& x^{\prime}=\frac{r-R^{\prime}}{a^{\prime}} \\
& h(r)=\left[1+\exp \left(\frac{r-R_{s}}{a_{s}}\right)\right]^{-1}
\end{aligned}
$$

The Coulomb potential is defined as

$$
\begin{array}{ll}
V_{c}(r)=\frac{z_{I} z_{2} e^{2}}{2 R_{c}} & \left(3-\frac{r^{2}}{R_{c}^{2}}\right) \quad r<k_{c} \\
V_{c}(r)=\frac{z_{I} z_{2} e^{2}}{r} & r>R_{c}
\end{array}
$$

and the spin-orbit potential of the Thomas form is given by

$$
v_{s o}(r)=\left(v_{s o}+i W_{s o}\right)\left(\frac{h}{m_{\pi} c^{2}}\right)^{2} \frac{1}{r} \frac{d}{d r}[h(r)] \vec{i} . \vec{\sigma} \text { II-7 }
$$

We rote that Equation II-5(b) provides for and absorptive term strongly peaked near the nuclear surface.

The solution of Equation II-4 yields the $u_{z j}$ from which the $f_{i j}$ are easily computed. The $E_{2 j}$ may then be used to compute observables such as the differential crosssection

$$
\frac{d \sigma}{d !}=\frac{1}{w} \sum_{v i d}\left|f_{U L}\right|^{2}
$$

where $w$ is the total number of spin states. Comparison of the calculated differential cross section with data yields a chi-squared parameter which may be minimized by successive 
repetitions of the same calculation whlle varying components of the potentfals and/or shape functions. The resuits of such calculations are presented in Chapter VI of this work.

since we are performing caicuiations for incident kinetic energies of $490 \mathrm{MeV}$ and higher, corresponding to a vaiue for $v / c$ of 0.713 , it is appropriate to use reiativistic kinematics. At such high bombarding energies it is reasonabie to expect a iarge number of ineiastic channels to be open, and thus for the imaginary part of the potential to dominate the scattering, indicating strong absorption. We might also expect the imaginary potential to increase in strength as the bombarding energy increases, refiecting the opening of more ineiastic channeis and an increase in the strength of some existing channeis reiative to the eiastic channel. Severai authors 28,29$)$ have noted that the reiativeiy weak reai centrai potentiai shouid change from attractive to repuisive at an incident energy of about 400 Mev. Such an observation is consistent with the behaviour of the nucieon-nucieon force as a function of energy, and is indeed observed. An exceiient summary of the opticai modei may be found in Jackson30) and references therein.

B) The Distorted Wave Impuise Approximation

The analysis of ineiastic transitions presented in Chapter VI of this work has its basis!in the Distorted wave Impulse Approximation (DWIA). We endeavour to present here 
a description of the salient features of the DWIA in order that the results of calculationg presented in Chapter VI may be more meaningful.

The impuise approximation ${ }^{2}$ ) may be understood as foilows. The many-body transition operator I may, foliowing KMT, be successiveiy approximated by

$$
T=\sum_{j} t(j) \simeq \sum_{j} \tau(j)
$$

$I I-9$

where $t(j)$ is the effective two-nucleon operator within the nuciear medium and $\tau(j)$ is the free two-nucieon operator describing the scattering of the lncident particie from the j'th nucleon. The basic assumption, then, is that the complex interaction may successfuliy be approximated by the free nucleon-nucieon t-matrix.

Furthermore, for $\varepsilon_{p} \geq 400$ MeV the energy of the incident projectile is ruch greater than that of the nucieons confined within the nucleus, and the interaction time with a barget nucieon is small compared with the characteristic period of a bound nucleon. Thase conditions comprise the basis for the Impulse Approximation. It shouid be pointed out that since the energy-momentum conditions for nucieonnucleus scattering are different from free nucieor-nucleon scatterirg the impuise approximation matrix elements are necessarily off the eruguy shell for free scattering.

The distorted waves representing the incident and scattered particles are plarie waves which are distorted in 
the entrance and exit channels by the effect of the elastic scattering optical potential In this work, both the incoming and outgoing projectiles are protons. In addition, the energy loss in the target is small with respect to the particle energy and consequently the incident and exit channel optical potentials must be the same, except for the small disterencies in energies. Since potentials change slowly with energy, this may be neglected. This potential, then, is a prerequisite for a distorted wave anaiysis of inelastic scattering.

The formalism of the DWIA has been wellcocumented ${ }^{2,29)}$. The transition matrix element for inelastic scattering from an initial state $i$ to a definite final state $f$ is given by

$$
I_{E i}\left(\vec{k}^{\prime}, \vec{k}\right)=\left\langle\Phi_{E} x_{f}^{-} \mid i_{j} \tau(j) ; \beta_{i} x_{i}^{+}\right\rangle \quad \text { II }-10
$$

where $x^{ \pm}$are the distorted waves for the projectile. As in the optical model, the distorted waves may be expanded in a partial wave expansion with appropriate angular romentum couplings and the transition matrix element may be evaluated using the effective interaction derived in the next section.

C) An Effective Nucleon-Nucieon interaction for Intermediate Energy Scattering

As we have seen, the DWIA requires the evaluation of a matrix element based on a realistic nucleon-nucleon in- 
teraction. which is also assumed to be able to describe with some degree of success nucleon-nucleus scattering. The effective interaction used in the DWIA calculations presented in this work is that which has recentiy been developed by Love and Eraney ${ }^{3)}$. We present here a summary of the Important features of this interaction, following the formalism as developed in their paper.

The starting point of the analysis is the free nucieon-nucleon scattering ampitude $M$, which may be obtained from a phase shift anaiysis as described by Mackregor et. ai. ${ }^{31)}$. Ampittudes at 425 and $515 \mathrm{MeV}$ were obtained Erom the phase shift aralysis of Bugg et. al. ${ }^{321}$, while at 650 and $800 \mathrm{MeV}$ the analysis of Arndt et. al.33,34) was used. Following $\mathrm{KMT}^{2}$, the nucieon-nucieon amplitudes may be expressed as

$M(E, q)=A+B \vec{\sigma}_{1} \cdot \hat{n}_{\vec{\sigma}} \cdot \hat{n}+C\left(\vec{\sigma}_{1}+\vec{\sigma}_{2}\right) \cdot \hat{n}+E \vec{\sigma}_{1} \cdot \vec{q} \vec{\sigma}_{2} \cdot \vec{q}+E \vec{\sigma}, \cdot \vec{Q} \vec{\sigma}_{2} \cdot \vec{Q} \quad$ II $-1 I$ where the coefficients $A, B, C, E$ and $F$ are functions of $E$, $q$, and $T$, the centre-of-mass energy, momentum transfer, and total two-body isospin respectiveiy. The spinors $\sigma_{1,2}$ and represent the spins of the incident and scattered particies respectively. If $\vec{k}, \vec{k}$ are the initiaj and final momenta of elther particle in the centre-of-mass system, then tre unit vectors $[\hat{q}, \hat{Q}, \hat{n}]$ form a right-handed coordinate system such that

$$
\begin{array}{lll}
\vec{q}=\vec{k}-\vec{k}^{\prime} & \vec{Q}=\vec{k}+\vec{k}^{\prime} & I I-12(a) \\
q=2 k \sin \frac{\vec{a}}{2} & 2=2 k \cos \frac{\theta}{2} & I I-12(b)
\end{array}
$$


To make the 1 sospin dependence explicit it is customary to express each coefficient as, for exampie,

$$
A=A_{x}+A_{3} \vec{E}_{0} \cdot \vec{E}_{j}
$$

where $\vec{t}_{0}, \vec{t}_{j}$ are isospin operators for nucieons 0 and $j$ respectively. In order to better understand the expression for $M$ it is convenient to reduce it using the identity

$$
\vec{z}_{1} \cdot \mathrm{Q} \vec{\sigma} \vec{\sigma}_{2} \cdot \hat{u}=\frac{1}{3}\left[\mathrm{~S}_{12}(\hat{\mathrm{u}})+\vec{J}_{1} \cdot \vec{J}_{2}\right] \quad \text { II-I4 }
$$

and the completeness condition on $[q, Q, n]$. The result is

$$
M(E, g)=A^{\prime} P_{S}+B^{\prime} P_{T}+C\left(\vec{s}_{1}+\vec{z}_{2}\right) . \hat{z}+E^{\prime} S_{12}(\hat{q})+E^{\prime} S_{12}(Q) \quad \text { II }-15
$$

where $S_{12}$ is the standard tersor operator, $P_{S}$ and $P_{t}$ are the spin singlet and tripiet projection operators respectively, and

$$
\begin{array}{lll}
A^{\prime}=A-B-E-F & B^{\prime}=A+[(B+E+E) / 3\} & I I-16(a) \\
E^{\prime}=(E-B) / 3 & E^{\prime}=(E-B) / 3 & I I-16(b)
\end{array}
$$

The expression has thus been reduced to centrai singiet and tripiet terms, a spin-orbit term and two tensor terms. The ampiticudes may be convereed into th nucieon-nucieon tmatrix by

$$
t_{N N}(E, q)=\frac{-4 T(h c)^{2}}{E} M(E, g) \quad E^{2}=\pi^{2} \Sigma^{4}+(K c k)^{2} \quad I I-I 7
$$

The point now is to relate the free nucieon-nucieon t-matrix so derived to an effective interaction capabie of describing a nucieon-nucieus interaction. It is convenient to begin this analysis by defining an effective nucleon-nucieon in- 
teraction which may then be miltipiled by an approprlate kinematic factor to yleld an effective nucieon-nucleus interaction. In each nucleon-nucieon channei the effective nucieon-nucieon interaction $V_{12}$ is represented by

$$
V_{12}=V^{C}\left(r_{12}\right)+V^{I S}\left(I_{12}\right) \vec{I} \cdot \vec{S}+V^{T}\left(r_{12}\right) S_{12}
$$

where $\vec{L} . \vec{S}$ and $S_{12}$ are the standard spin-orbit and tensor operators. The parameters of $V_{12}$ are then adjusted to optimize the representation

$$
t_{N N}(E, q)=\int d^{3} r e^{-i \vec{k}^{\prime} \cdot \vec{r}_{V}}{ }_{12}\left[I+(-)^{\ell} \underline{p}^{x}\right] e^{i \vec{k} \cdot \vec{r}} \quad \text { II-19 }
$$

where $\mathrm{p}^{\mathrm{X}}$ is the spatiai coordinate exchange operator operating to the right and $(-)^{l}$ ensures antisymmetrization. The anguiar momentum is the reiative angular momentun in the nucieon-nucieon system. To provide some physical insight into the structure of the coefficients in the expression for $V_{12}$, and to ensure compatibiilty with the computer code DHBA7g, the radial parts of $V^{C}$ and $V^{\text {LS }}$ may be represented by a sum of Yukawa terms while the radiai shape of $v^{T}$ is taken to be $r^{2}$ times a sum of Yukawa terms. Conseguently

$$
\begin{aligned}
V^{C}(r) & =\sum V_{i}^{C} Y\left(\frac{r}{R_{i}}\right) \\
V^{L S}(r) & =\sum V_{i}^{L S} Y\left(\frac{r}{R_{i}}\right) \\
V^{T}(r) & =\left[V_{i}^{T} I^{2} Y\left(\frac{r}{R_{i}}\right)\right. \\
Y(x) & =\left[e^{-x}\right] / x
\end{aligned}
$$$$
\begin{aligned}
& I I-20(\mathrm{a}) \\
& I I-20(\mathrm{~b}) \\
& I I-20(\mathrm{c}) \\
& I I-20(\mathrm{~d})
\end{aligned}
$$

For the reai part of $V^{c}$ the iongest range is chosen to be representative of the iong-range part of the one-pion exchange potentiai (OPEP). The maximum range of 0.7 fm for $\mathrm{V}^{T}$ 
was chosen so as to emulate the smail q Fourler components of the tensor part of the OPEP. The range parameter of 0.4 fin was chosen to roughiy represent the effects of multipie pion-exchange processes. The remaining range parameters were chosen for fiexibility.

Recalling that $t_{N N}$ is related to $v_{12}$ by a Fourier transform (see Eq. II-19), some aigebra yields the foilowing reiations between the t-matrix components and the ampiitudes:

$$
\begin{array}{rlrl}
t_{s}^{C} & =\tilde{V}_{S}^{C}(q)+(-)^{2} \tilde{V}_{s}^{C}(Q)=n A^{\prime} & I I-2 I(a) \\
t_{T}^{C} & =\tilde{V}_{T}^{C}(q)+(-)^{2} \tilde{V}_{T}^{C}(Q)=n B^{\prime} & I I-2 I(b) \\
4 t^{L S} & =\tilde{Q V}^{L S}(q)-(-)^{2} q \tilde{V}^{I S}(Q)=-4 i n C & I I-2 I(c) \\
t^{T} & =\tilde{V}^{T}(q)=-n F^{\prime} & I I-2 I(d) \\
V^{T}(Q) & =(-)^{i+1} n F^{\prime} & & I I-2 I(e)
\end{array}
$$

symmetry requires

$$
E^{\prime}(\pi-\varepsilon)=(-)^{2} F^{\prime}(\theta)=(-)^{T} F^{\prime}(\theta) \quad \therefore \quad I I-22
$$

The nucieon-nucieus t-matr $1 x$ is then given by $t_{N A}$ where

$$
t_{\mathrm{NA}}=\frac{\varepsilon_{0}^{2}}{\varepsilon_{p} \varepsilon_{t}} t_{\mathrm{NN}}
$$

In this expression $\Sigma_{c}, \varepsilon_{p}$ and $\Sigma_{t}$ are energies representing the total energy of the incident nucieon in the nucieonnucieon system, the total energy of the incident nucieon in the nucieon-nucieus system and the totai energy of the target nucieon in the nucieon-rucleus system respectiveiy.

It is instructive to examine the dependence of the 
t-matrlx on the exchange term in the nucleor-nucieus system. For a given nucleon-nucleon channel the $t$-matrix may be written in a Fourter transform representation as the sum of a drect term and an exchange term,

$$
t_{N N}=\tilde{v}(q)+\tilde{v}(Q) ; Q^{2}=4 k^{2}-q^{2}
$$

Petrovich et. al. ${ }^{35}$ and Love ${ }^{36)}$ have shown that for the nucleon-nucieus system it is reasonable to approximate the exchange term as

$$
t_{N N}=\tilde{v}(q)+\tilde{v}\left(k_{A}\right)
$$

where $k_{A}$ is the momentum of the incident nucieon in the centre-of-mass. Physlealiy, the exchange term represents the ejection of a proton which is not the proton which colilded with the nucieus in the first instance. Consequently, the momentum transfer required for this term is that associated with stopping the incident nucieon. The exchange terms are evaiuated in a short-range approximation described by petrovich et. 35) al. and Raynal ${ }^{37)}$. The exchange terms are included explicitly in the components of the t-matrix.

Having thus determined the effective interaction, it is now appropriate to relate the eiements of the interaction and amplitudes to physical observables. Such relations will allow investigation of the dependences of observabies on components of the interaction for different classes of nueiear transitions. It is these dependences we investigate In more detall in Chapter VI. 
For natural parity excitations $\left(\Delta \pi=(-)^{J}, \Delta S=\theta\right)$ in the Plane Wave Impulse Approximation, Kerman, McManus and Thaler ${ }^{2}$ have shown that the differential cross-section for excitation of a state of definite $J$ in terms of the nucieon-nucieon anplitudes is

$$
\frac{d \sigma}{d \Omega}=\left[|A|^{2}+|C|^{2}\right] * S F
$$

where SF is a nuclear structure factor. For unnatural parity excitations $\left(\Delta \pi=(-)^{\mathrm{J}+1}, \Delta S=1\right.$ dominant $)$ they find

$$
\frac{d \sigma}{d \Omega}=\left[|B|^{2}+|C|^{2}+|F|^{2}+\eta|E|^{2}\right] * S F \quad I I-27
$$

where $\eta$ is a structure factor dependent on the relative $1 m-$ portance of aijowed orbital anguiar momentum transfers in the generation of $\mathrm{J}$, and is defined by $2 \mathrm{~J} /(\mathrm{J}+1)$ for $\mathrm{L}=\mathrm{J}-1$.

In terms of the isospin and exchange dependent representations of the nucieon-nucieon t-matrix, these expressions become

$$
\frac{d \sigma}{d s}=\left[\left|t^{C}\right|^{2}+: t^{L S ! 2}\right] * S F
$$

for natural parity states and

$$
\frac{d \sigma}{d \Omega}=\left(\left|t^{L S}\right|^{2}+\left|t^{C}+t^{T \alpha_{1}{ }^{2}}+t^{C}+t^{T 3}\right|^{2}+7 \cdot t^{C}+\left.t^{T \gamma}\right|^{2}\right\}^{* S F}
$$

for unnatural parity states where

$$
\begin{aligned}
& t^{T \alpha}=t^{T}(q)+(-)^{2} t^{T}\left(k_{A}\right) \\
& t^{T \beta}=-2 t^{T}(q)+(-)^{2} t^{T}\left(k_{A}\right) \\
& t^{T \gamma}=t^{T}(q)-2(-)^{2} t^{T}\left(k_{A}\right)
\end{aligned}
$$$$
I I-30(c)
$$

represent the tensor contributions to $B, F$ and E respectiveiy. 
Now it must be pointed out that these equations are stili not representative of the nucieon-nucieus scattering since the components refer to states of definite spin and isospin in the nucleon-nucieon system. In nucieon-nucieus scattering, transfer of definite units of spin and isospin are appropriate and Love and Franey have derived expressions for such t-matrix elements in terms of the components of the nucieon-nucleon t-matrix. The final expresions are then

$$
\begin{aligned}
& t_{0}^{C}=\frac{1}{16}\left[3 t^{S E}+3 t^{T E}+t^{S O}+9 t^{T O}\right] \\
& t_{\sigma}^{C}=\frac{1}{16}\left[-3 t^{S E}+t^{T E}-t^{S O}+3 t^{T O}\right] \\
& t_{\tau}^{C}=\frac{1}{16}\left[t^{S E}-3 t^{T E}-t^{S O}+3 t^{T O}\right] \\
& t_{\sigma \tau}^{C}=\frac{1}{16}\left[-t^{S E}-t^{T E}+t^{S O}+t^{T O}\right] \\
& t_{0}^{L S}=\frac{1}{4}\left[t^{L S E}+3 t^{L S O}\right] ; \quad t_{\tau}^{L S}=\frac{1}{4}\left[-t^{L S E}+t^{L S O}\right] \quad I I-3 I(e) \\
& t_{0}^{T}=\frac{1}{4}\left[t^{T N E}+3 t^{T N O}\right] ; \quad t_{T}^{T}=\frac{1}{4}\left[-t^{T N E}+t^{T N O}\right] \text { II-3I(E) }
\end{aligned}
$$

and for exampie

$$
t_{\tau}^{T \alpha}=\frac{1}{4}\left[-\left[t^{T N E}(q)+t^{T N E}\left(k_{A}\right)\right]+\left[t^{T N O}(q)-t^{T N O}\left(k_{A}\right)\right]\right\} I I-32
$$

In terms of these components, states of naturai parity depend upon $t_{0}^{C}$, and $t_{0}^{L S}$. For unnaturai parity transitions only the spin-transfer dependent parts of the central terms contribute, together with $t^{\text {LS }}$ and $t^{T}$ where

$$
\left|E^{T}\right|=\left[\left|t^{T \alpha}\right|^{2}+\left|t^{T B}\right|^{2}+n\left|t^{T Y}\right|^{2}\right]^{l / 2}
$$

The analyzing powers may in turn be expressed as

$$
A_{y}=\frac{2 \operatorname{Re}[A * C]}{\left[|A|^{2}+|C|^{2}\right]}
$$


for natural partty transitions and

$$
A_{y}=\frac{2 R e\left[B^{*} C\right]}{\left.\left.|| B\right|^{2}+|C|^{2}+|E|^{2}+\eta|E|^{2}\right]}
$$

for unnatural parity transitions. Rewriting these expressions in terms of the appropriate nucleon-nucleus t-matrix elements we find

$$
A_{y}=\frac{2\left[t_{R}^{L S_{T}} t_{I}^{C}-t_{I}^{L S} t_{R}^{C}\right]}{\left|t^{C}\right|^{2}+\left|t^{L S}\right|^{2}}
$$

for natural parity transitions and

$$
A_{Y}=\frac{2\left[t_{R}^{L S}\left(t_{I}^{C}+t_{I}^{T x}\right)-t_{I}^{L S}\left(t_{R}^{C}+t_{R}^{T \alpha}\right)\right]}{\left|t^{L S}\right|^{2}+i t^{C}+\left.t^{T \alpha}\right|^{2}+t t^{C}+\left.t^{T S}\right|^{2}+7 i t^{C}+\left.t^{T Y}\right|^{2}} \text { II-37 }
$$

for unnatural parity transitions.

Note that if the product of $\frac{d \sigma}{d \Omega}$ and $A_{y}$ is taken, then the denominators in the expressions for A $y$ reduce to unity and we simply find for natural and unnaturai parity transitions respectiveiy,

$$
\begin{aligned}
& \frac{d C}{d i} * A_{y} \propto 2 \operatorname{Re}[A * C]=2\left(t_{R}^{L S} t_{I}^{C}-t_{I}^{L S} t_{R}^{C}\right] \quad I I-33(a) \\
& \frac{d \sigma}{d \Omega} * A_{y} \propto 2 R e[B * C]=2\left[t_{R}^{I S}\left(t_{I}^{C}+t_{I}^{T} \dot{i}\right)-E_{I}^{I S}\left(t_{R}^{C}+t_{R}^{T x}\right)\right]
\end{aligned}
$$

Note that these expressions as derived by KMT and Love and Franey are valid only in the Plane Wave Impuise Approximation. The effect of distortions must then be taken into account, and these will be diseussed in Chapter VI. Nevertheless, these expressions may be used as a guide to the sys- 
tematic dependence of the observables on efther the amplitudes or the components of the t-matrix.

In order. to better appreciate the reiative significance of the components of the nucleon-nucleus t-matrix, (inciuding exchange), Figures II-1 to II-12 present the dependence of each component in Equations II-3I on lncident nucieon energy and momentum transfer. Several general observations are in order.

The strongest energy dependence is seen, for the scaiar-isoserias term $t_{0}^{C}$, which is also seen to dominate for aij energies, espectaily for $q\left\langle 1 \mathrm{fm}^{-1}\right.$. For $q>1 \mathrm{fm}^{-1}$ the strengths of $t_{0}, t_{0}^{L S}, t_{0}^{T}$ and $t_{T}^{T}$ become comparable for $E_{p}<$ $400 \mathrm{MeV}$, while $t_{0}^{C}$ continues to dominate for $E_{p}>400 \mathrm{MeV}$. One might then expect that scalar-isoscalar excitations shouid dominate the nucieon-nucieus spectrum for smail vaiues of $q$. A notabie exception is the strong excitation of $1^{+}$states for small $q$, a point which wiil be discussed iater. The scaiar-1soscalar spin-orbit term grows in reiative importance as $q$ increases and should thus contribute significantiy to the excitation of isoscaiar naturai parity statas in regions of iarger momentum transfer. The 1sovector spin-orbit term is the weakest of aij terms in general, and may oniy contribute to the analyzing powers for some excitations. The centrai isoscaiar spin-filp term tf is generaily weak for ali $q$ and $E_{p}$. The isoscaiar tensor term $t_{0}^{T}$, which is dominated by exchange terms, is significant for al 


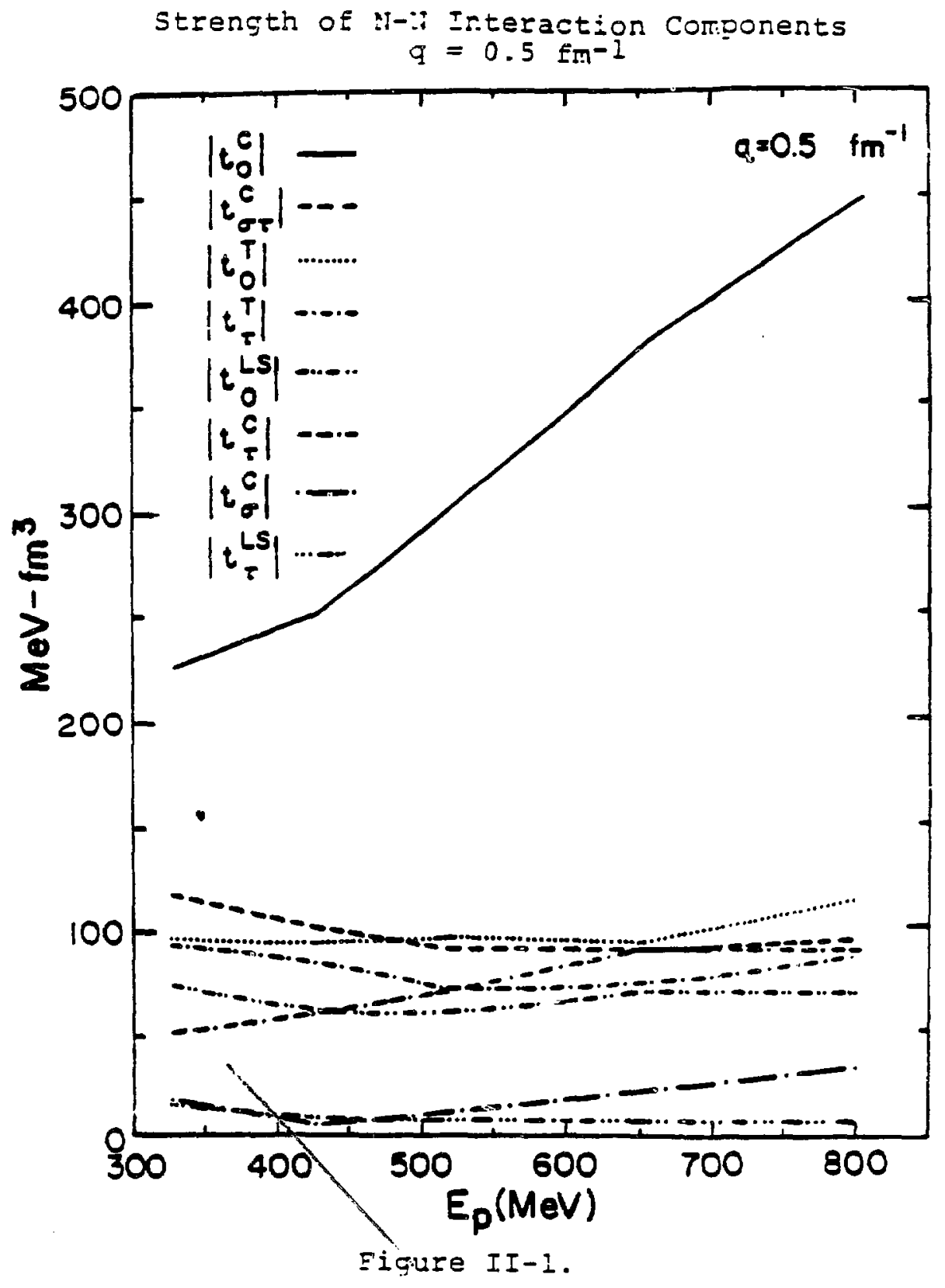




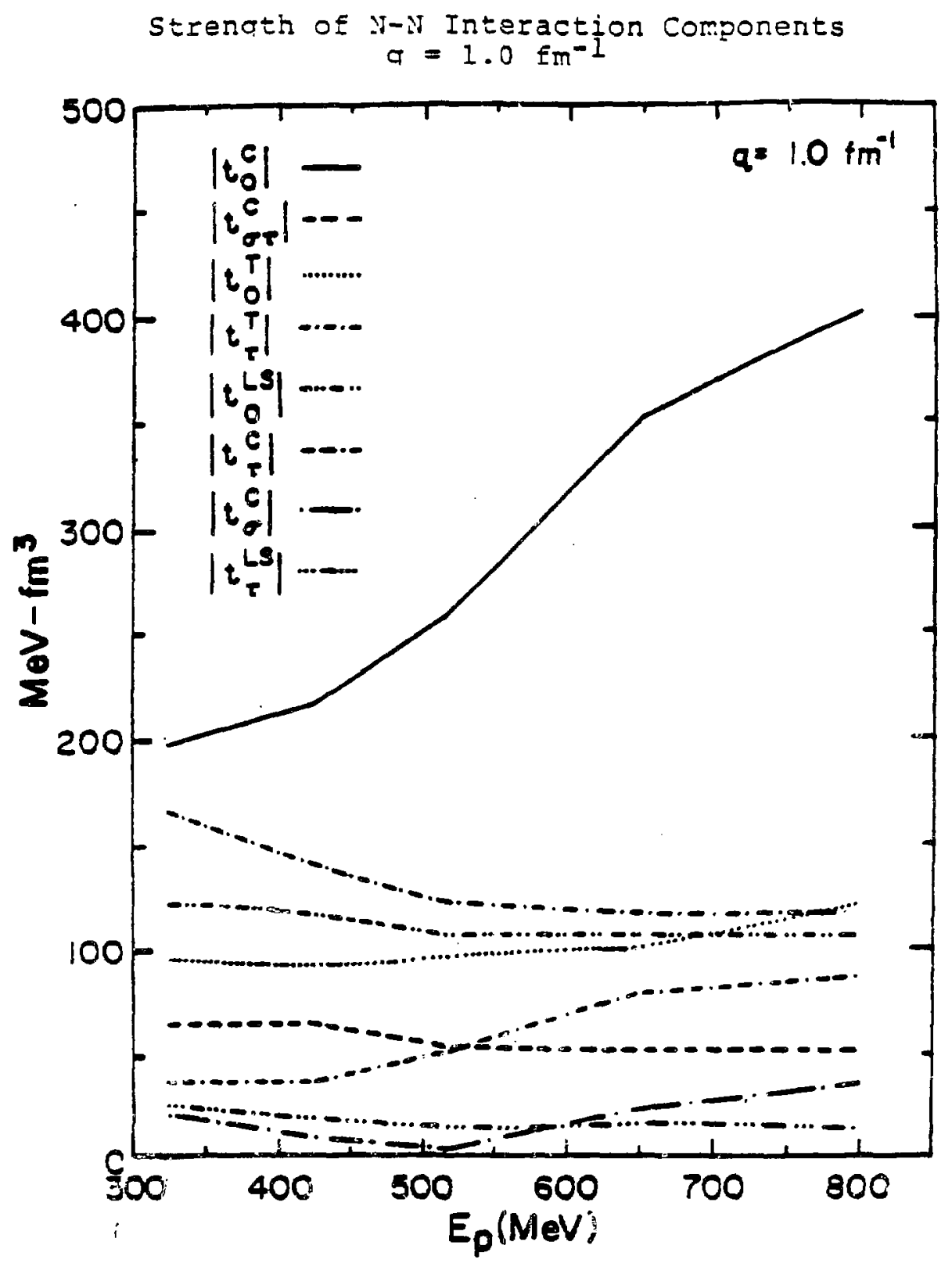

Figure II-2. 


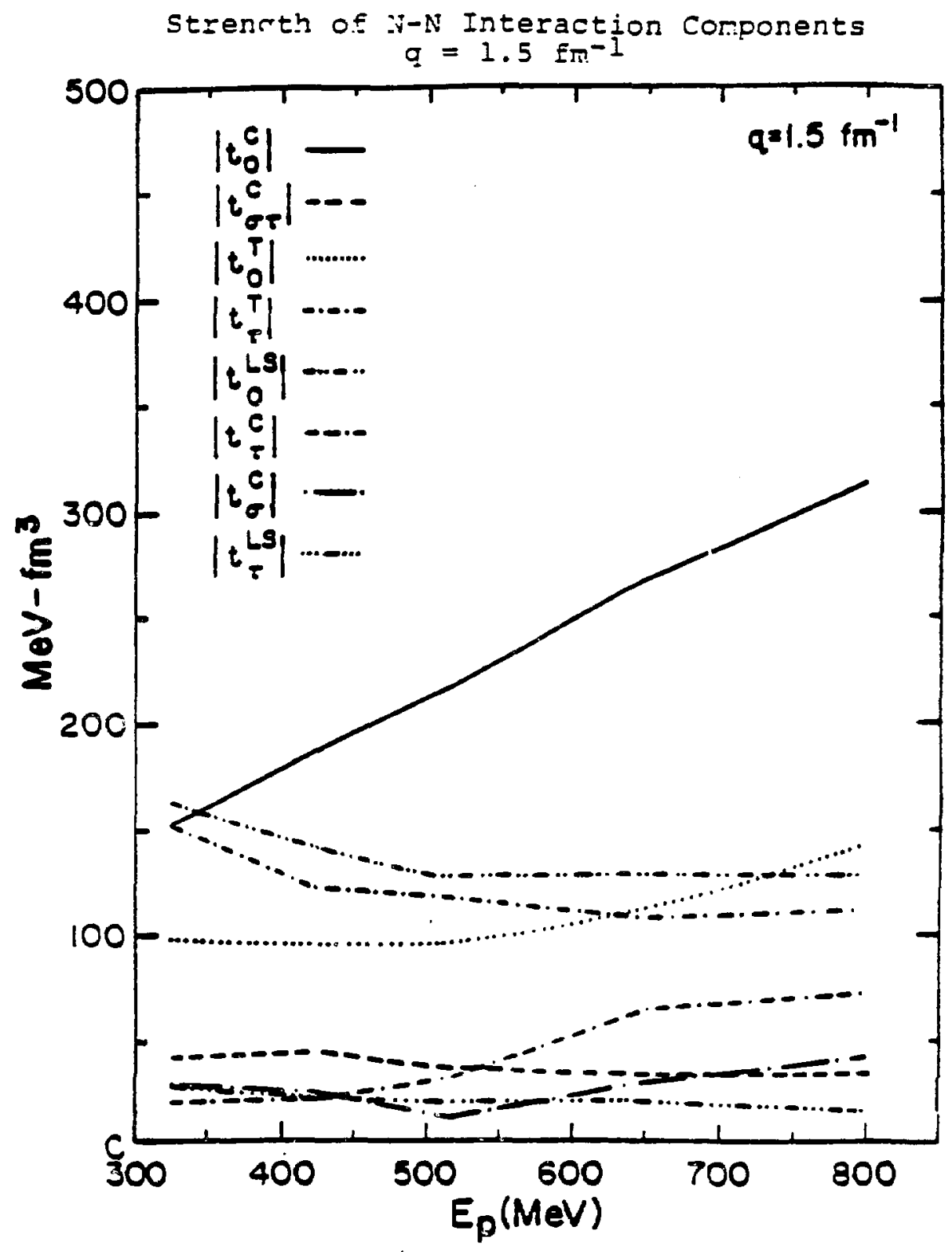

Eigure II-3. 


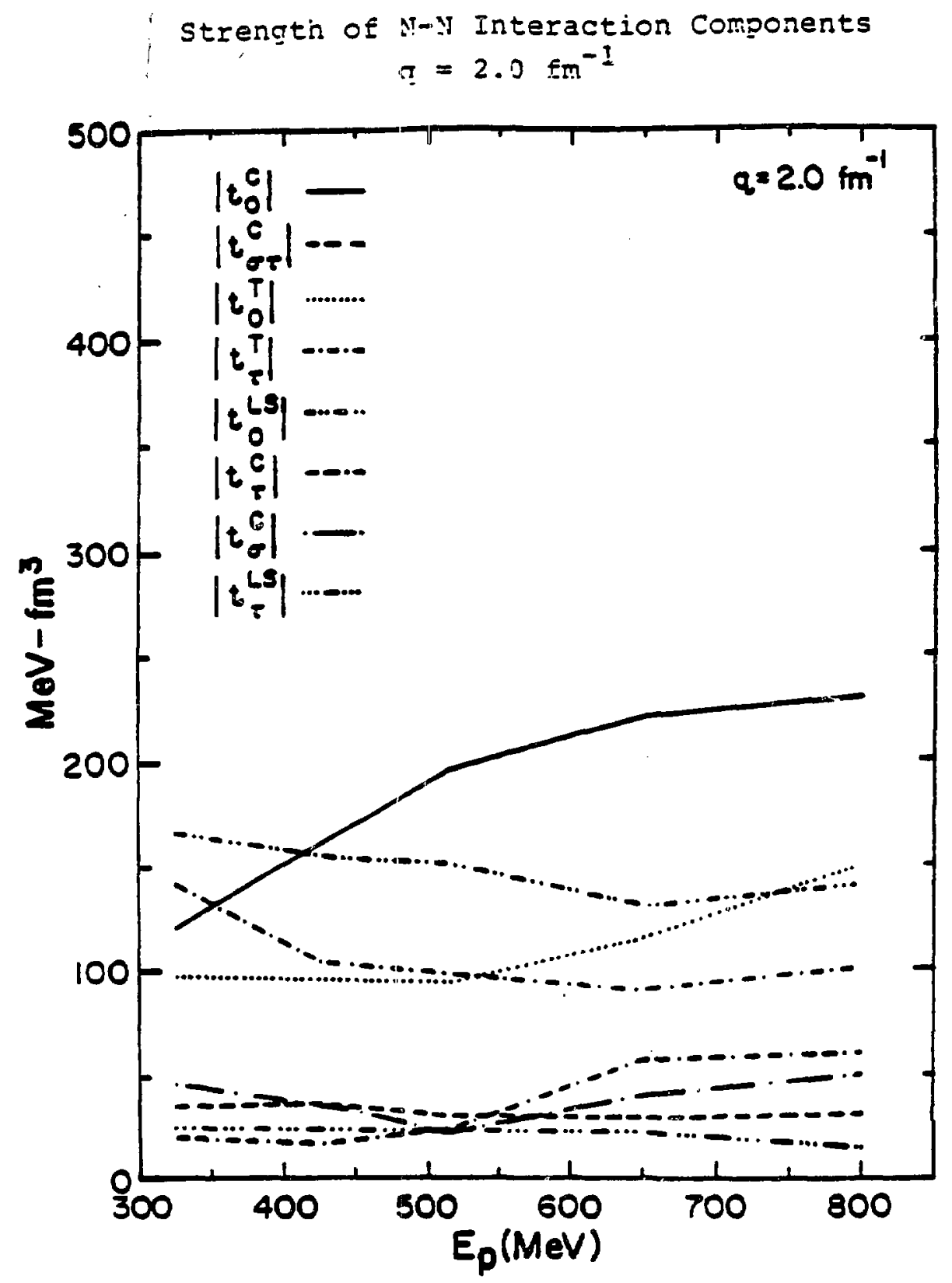

Eigure II-4. 


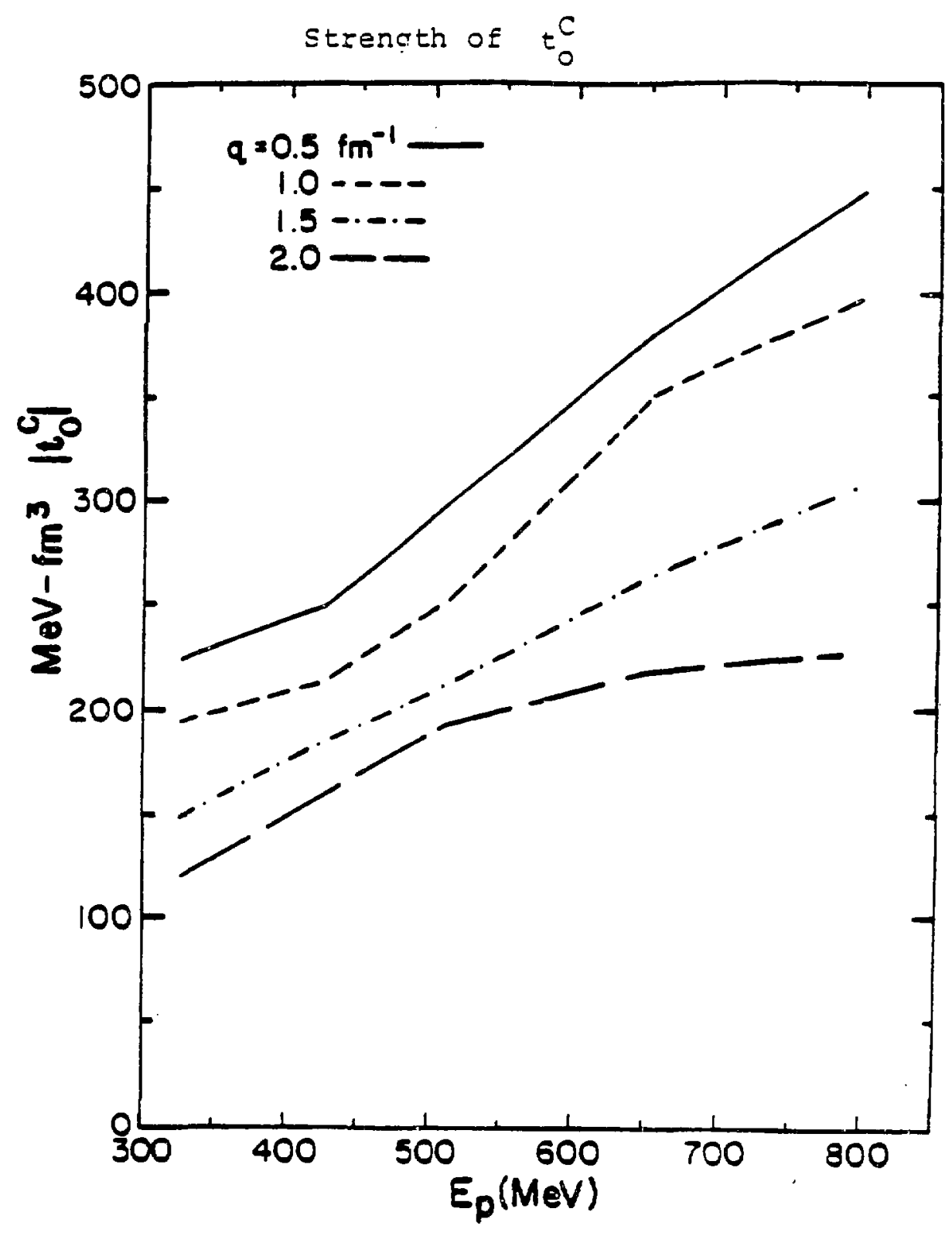

Eigure II-5. 


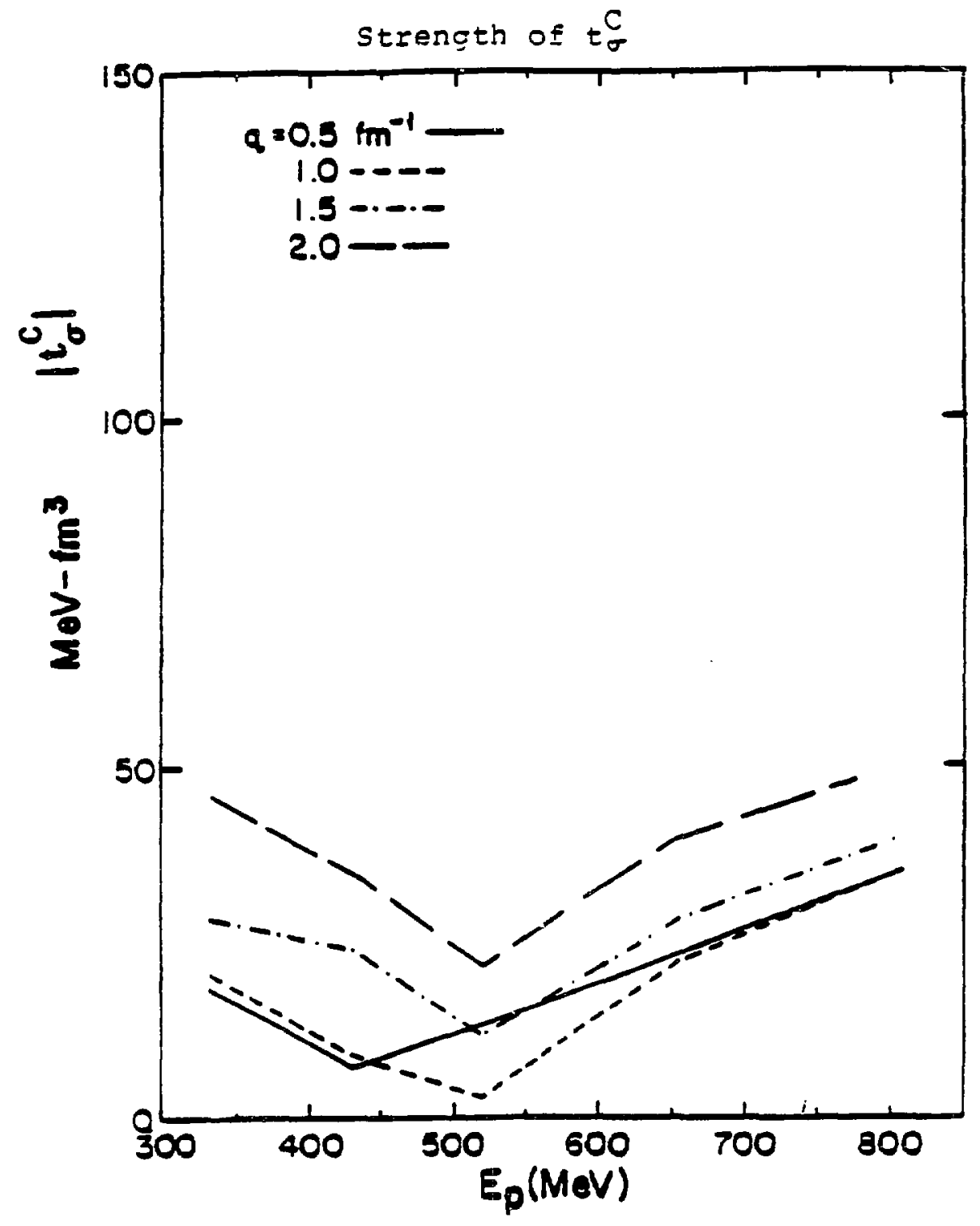

Eigure II-6. 


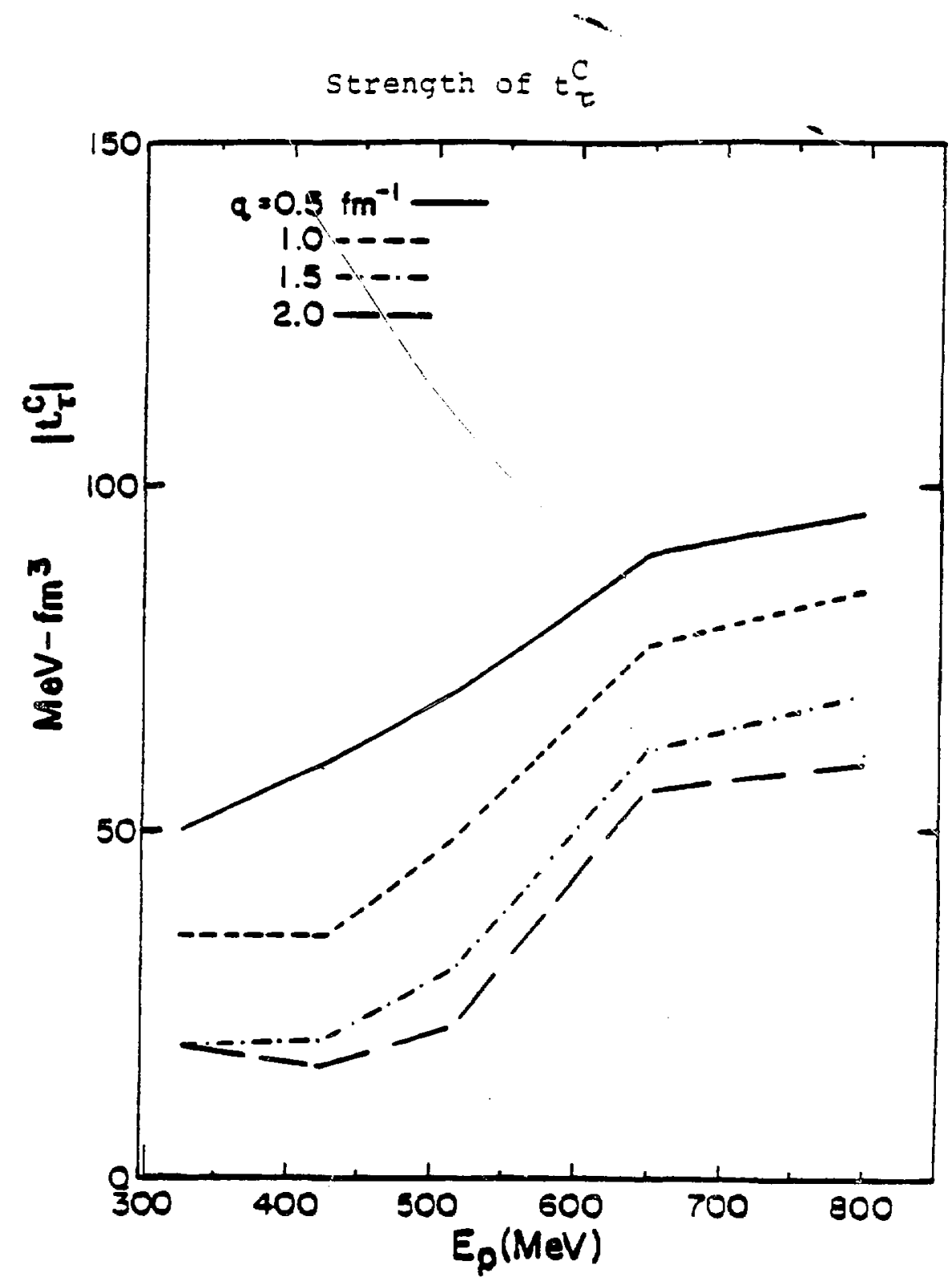

Figure II-7. 


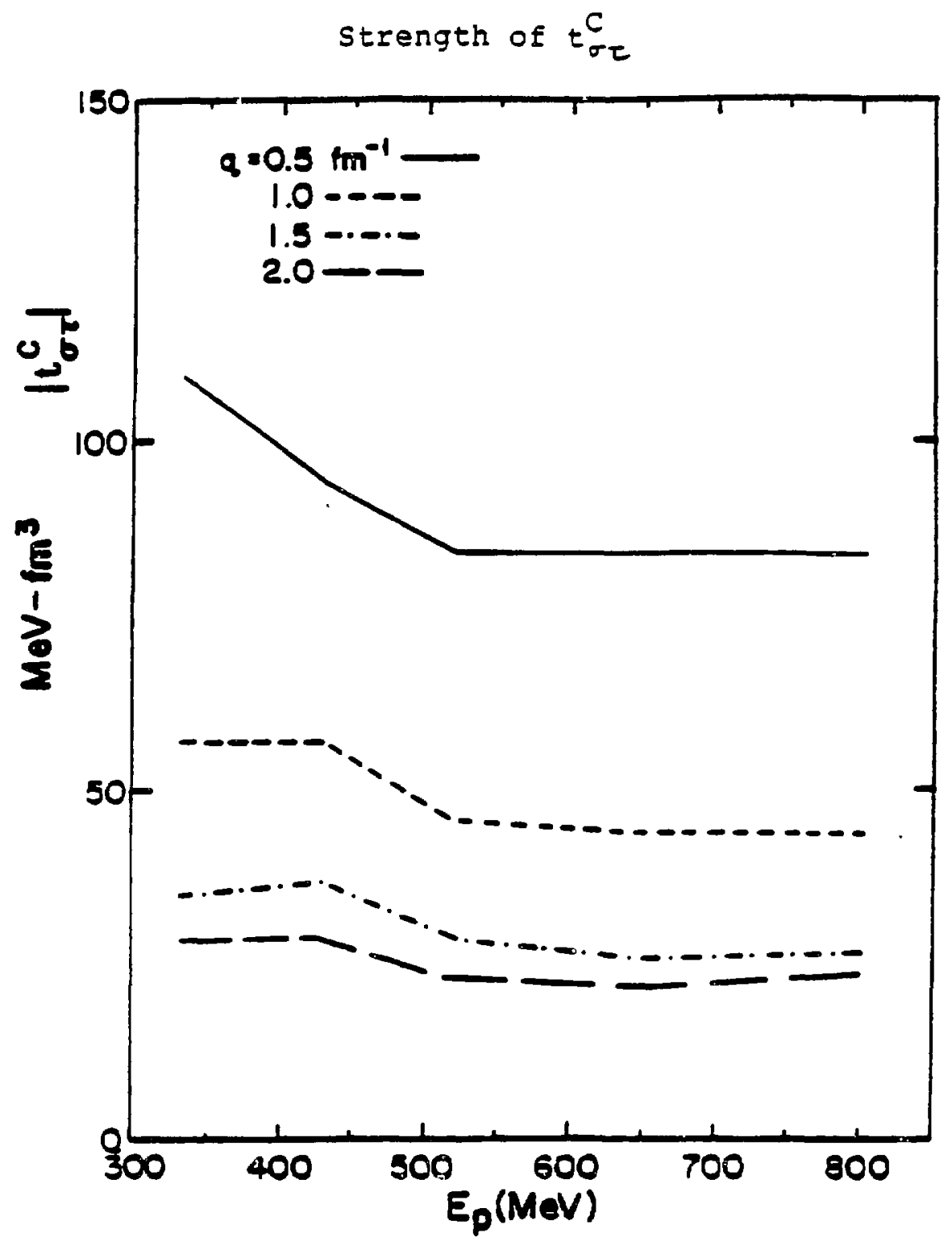

Figure II-8. 


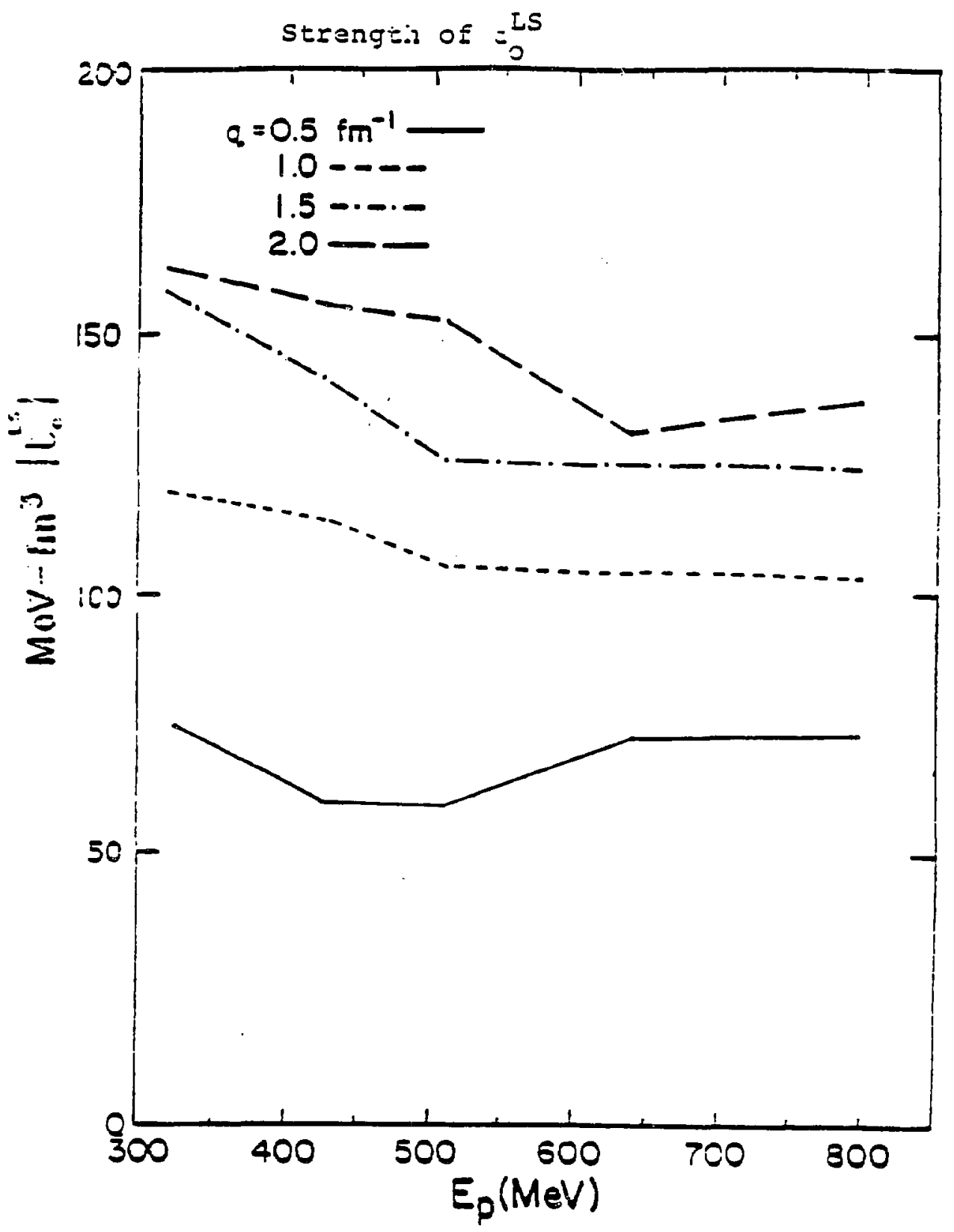

Eigure II-9. 


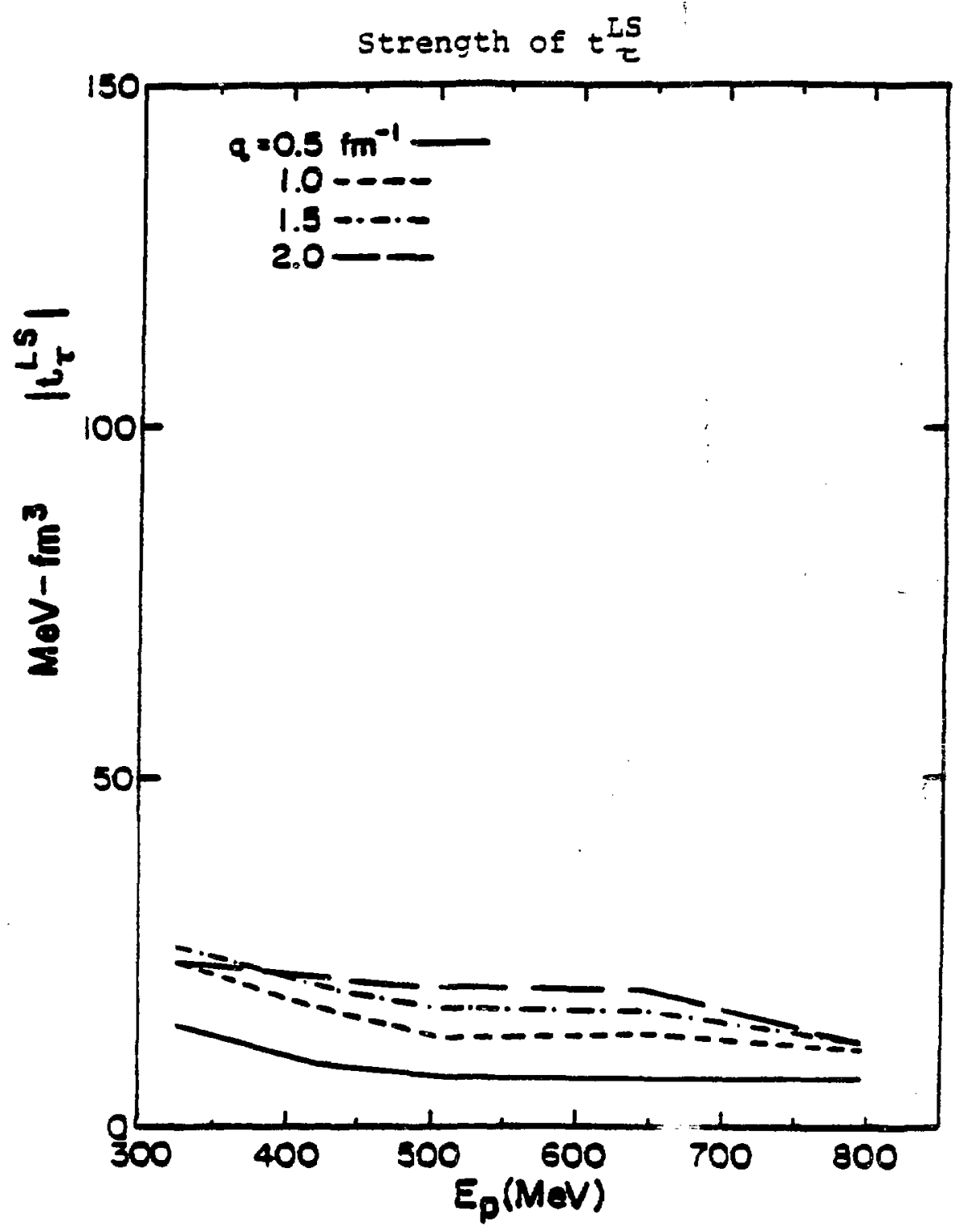

Figure II-10. 


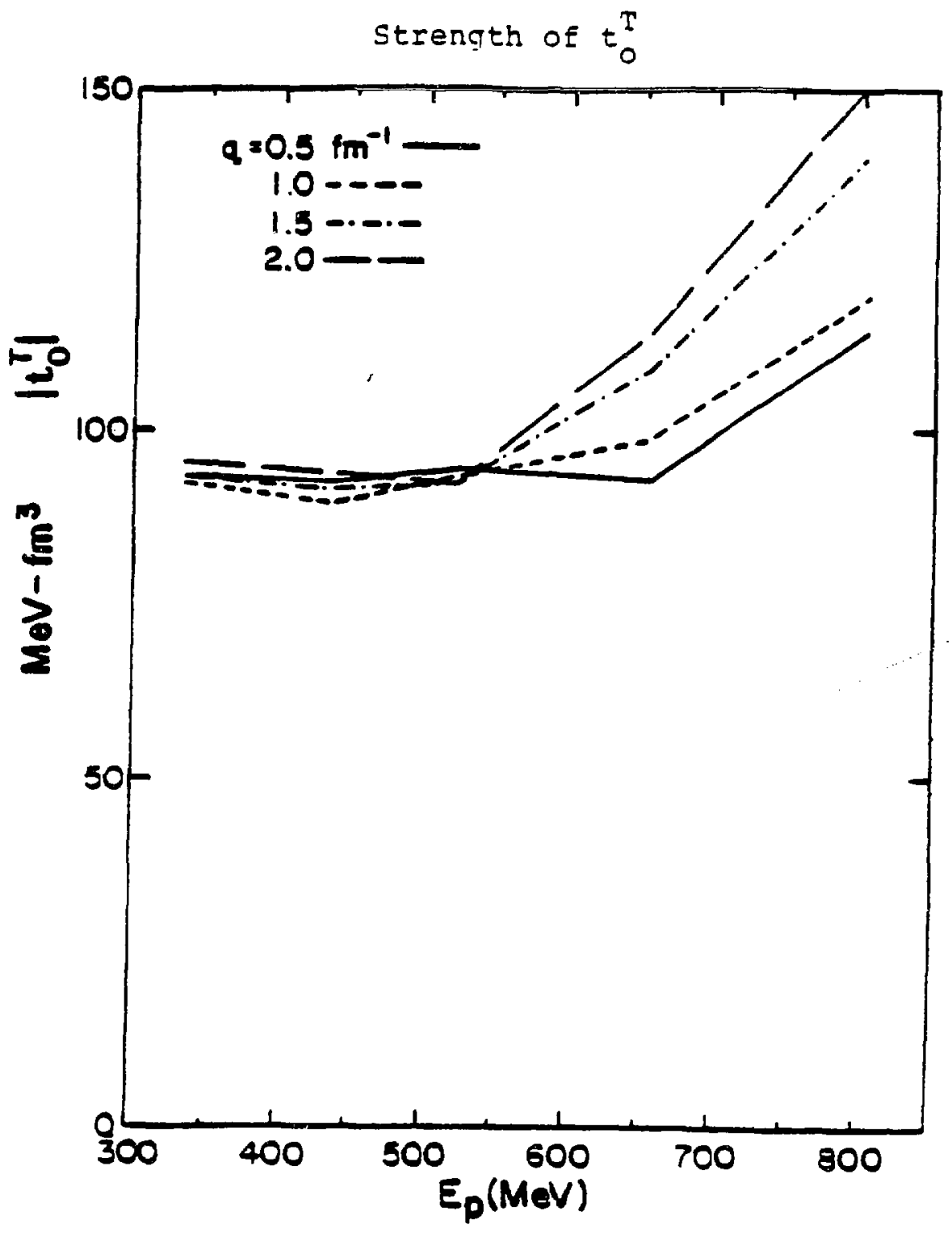

Eigure II-11. 


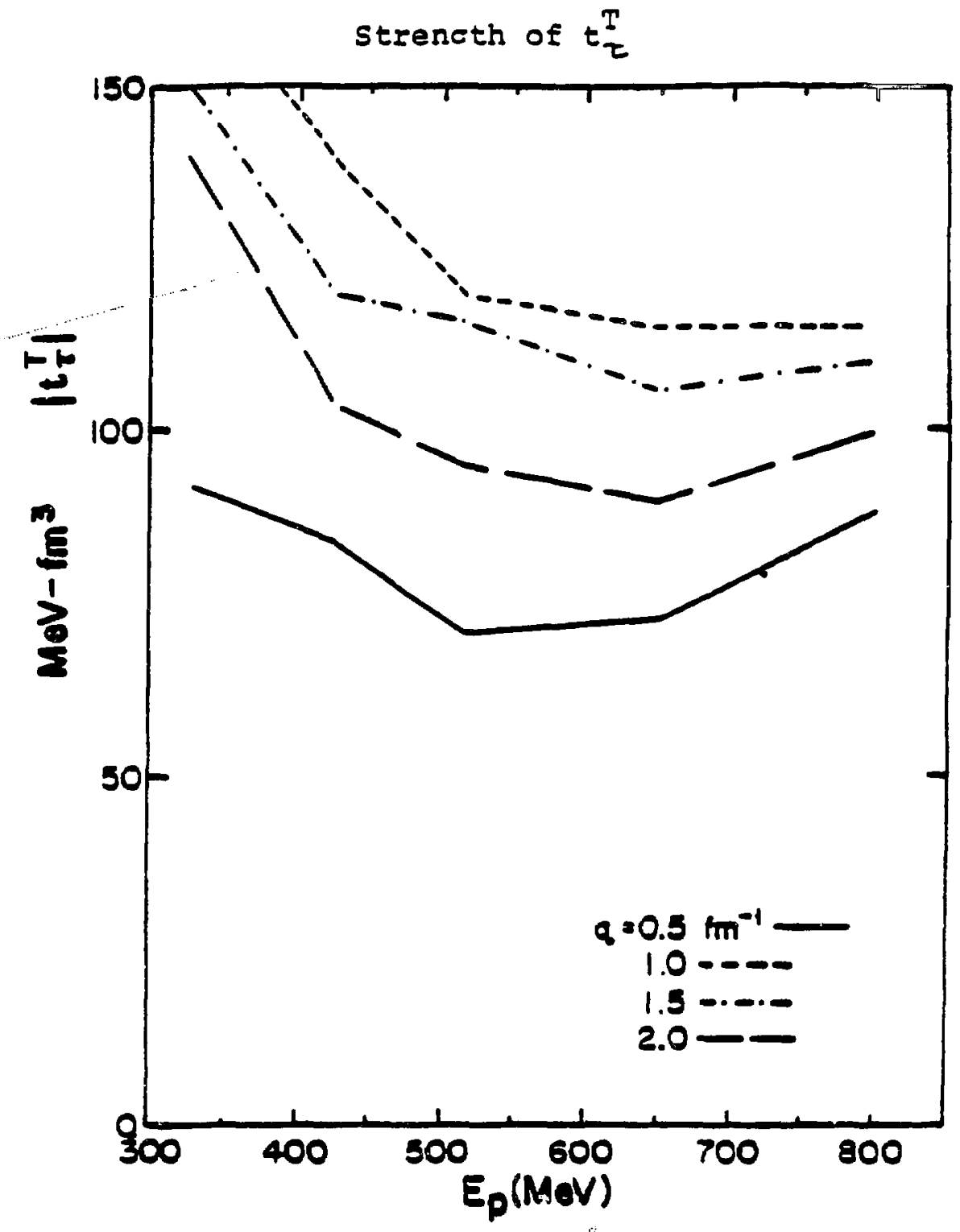

Figure II-12. 
$q$ and $E_{p}$, as is the isovector part $t_{T}^{T}$. The spin and 1sospin-filp component of the central part, $t_{z}$ is uniform as a function of incident particie energy, refiecting the dependence of the one-pion exchange potential which dominates this term. This term aiso is much stronger for smail momentum transfers than for iarge $q$.

The excitation of unnatural parity isovector states is expected to be dominated by $t_{\tau}^{c}$ for jow $q$, and by $t_{t}^{T}$ for iarge $q$. The reiative strength of the spin and isospln-filp term mentioned previousiy would then account for the strong excitation of $1^{+}$states at iow momentum transfer. For isoscaiar unnaturai parity states several terms are invoived, nameiy the $t_{\sigma}^{C}, t_{0}^{L S}$ and $t_{0}^{T}$ terms, with the tensor term dominating through exchange processes for smail 9 .

The estimations of the behaviour of anaiyzing powers are somewhat more compiex because of the dependence on reai and imaginary parts of the t-matrix eiements. For naturai parity isoscaiar transitions the dominant terms are $t_{I}^{C}$ and $t_{R}^{L S}$ which are both iarge and negative, resuiting in positive anaiyzing powers (see Eq. II-36). Naturai parity isovector excitations are found to have moderate positive vaiues at ail energies. Isovector states of unnaturai parity are predicted to have very smail anaiyzing powers. This refiects the fact that $\mathrm{C}^{C}$ and $t^{T}$ are predominantiy real, and aiso that $t^{\text {LS }}$ is very weak, especialiy in the imaginary part. Isoscalar transitions of unnatural parity are found 
to be negative for small $q$, changing to positive values as $q$ increases. This is dominated by the behaviour of $t_{0}^{\text {LS }}$ and $t^{C}$, and provides a potential unique signature for such transitions. 


\section{EXPERIMENTAL METHOD}

\section{A) Accelerator}

The data presented in this work were taken at the High Resoiution Spectrometer (HRS) faclitity at the CIinton P. Anderson Los RĨ anos Meson Physics Facility (LAMPF). Polarized negative hydrogen lons are produced in a Lamb-shift type polarized ion source, and are injected into the inear accelerator at an energy of $750 \mathrm{keV}$. This injection energy is provided by a Cockroft-Walton accelerator. The polarized negative hydrogen beam $(P-)$ is then acceierated to an energy of $1.30 \mathrm{MeV}$ in a four-sector Alvarez-type drift-tube inear accelerator operating a $201.25 \mathrm{~Hz}$. Further energy is provided by up to 48 sectors of a resonantiy-side-coupled standing-wave inear accelerator operating at $805 \mathrm{MHz}$.

Since the accelerator is primarily a plon production faciitity, a proton beam $(H+)$ is accelerated through aij radiofrequency sectors to a maximum energy of 300 MeV. The negative beam, P-, is acceierated on the opposite phase portion of the radiofrequency osciilation. In normai singie energy, duai beam operation at $800 \mathrm{MeV}$, both $\mathrm{H}+$ and $p$ - beams are puised at a frequency of $120 \mathrm{~Hz}$ with a puise iength of 500 microseconds, resuiting in a duty factor of 68 .

It is possibie, however, to reduce the energy of the P- beam while maintaining that of the H+ beam at $800 \mathrm{MeV}$. The experiments described here were among the first to util- 
1ze the dual energy beam capability of the LAMPF linear accelerator. This is accomplished in the following manner.

Two thirds of the $120 \mathrm{~Hz}$ macropulses are devoted exciusively to acceleration of the $\mathrm{H}+$ beam with an exterided pulse length of approximately 750 microseconds. The precise pulse length varies from one experimental cycle to another, depending upon optimum accelerator operation conditions. The resulting duty factor for the it beam is then close to 6\%, as it is in fuil dual-beam operation at $800 \mathrm{Mev}$. The remaining third of the pulses are devoted exciusively to acceieration of the p- beam on the approprlate phase of the radiofrequency osclilation. Two pulses of H+ beam are followed by one puise of $P$ - beam.

Since the $P$ - beam is now accelerated independently of the $\mathrm{H}+$ beam, it is possibie to control the energy of the negative beam by reducing the number of radiofrequency moduies operating in the $805 \mathrm{MHz}$ portion of the accelerator during the acceleration of the $p$ - beam. By selectively turning off some kiystrons it is possibie to obtain the beam energies of 398,597 and $698 \mathrm{MeV}$ used in this work. The puise frequency and duration of $4 \mathrm{~Hz}$ and about $750 \mathrm{mi}-$ croseconds result in a duty factor of close to 38 for the pbeam in the dual-energy mode of operation.

Upon exiting the accelerator the $p$ - beam is magneticaily separated from the $H+$ beam and deflected into line $x$, 
as shown in Flgure III-1 and III-2. The beam in ilne $x$ may be tallored by a series of strippers of varlous geometries and collimator jaws to provide acceptable momentum and spatial dispersion for Iines $B$ and $C$. Electrons are removed from that portion of the p-beam which passes through the strlppers, creating a mixture of $\mathrm{H}+$ and $\mathrm{P}$-beam. The str 1 pped and unstrlpped beams are then magneticaliy separated and the stripped Ht beam enters line $C$. The beam $1 \mathrm{~s}$ then eransported through the Iine C quadrupole-dipole system to the HRS target. The beam transport properties of I ine $c$ together with the beam dispersion matching conditions at the KRS target have been described in detall previousiy 38,39 ).

The beam spot on target was approximately $3 \mathrm{~mm}$ wide and 2 am high. The vertical dispersion of the beam is required for proper momentum dispersion matching with the spectrometer acceptance. The final beam on target was poiarized normal to the scattering piane with an average polarization of 0.80 as measured by the ine c polarimeter. The beam polarlzation was reversed every 3 minutes at the ion source to eliminate errors which may have resuited from systematic drifts in the beam poiarization.

Beam currents were adjusted by sejected coilimating jaws in ine $c$ to provide reasonabie event rates in the focai piane detector array of the HRS. Typicai beam currents ranges from tens of plcoamperes at forward angies where iarge count rates exist due to dominant eiastic scttering, 
Experimental Areas

Los Alamos Meson Physics Facility

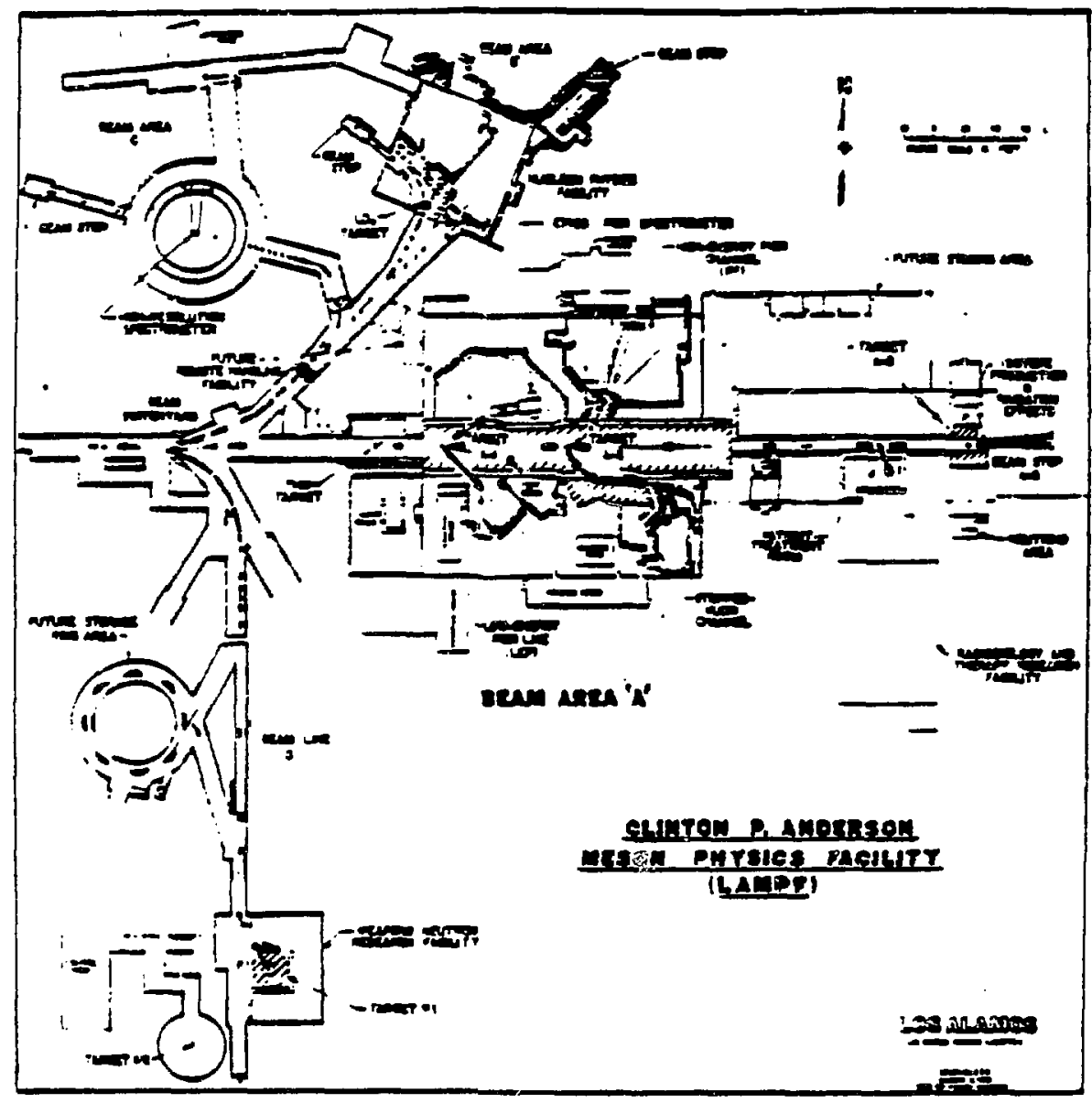

Figure III-1. 
Experimental Area ' $C$ '

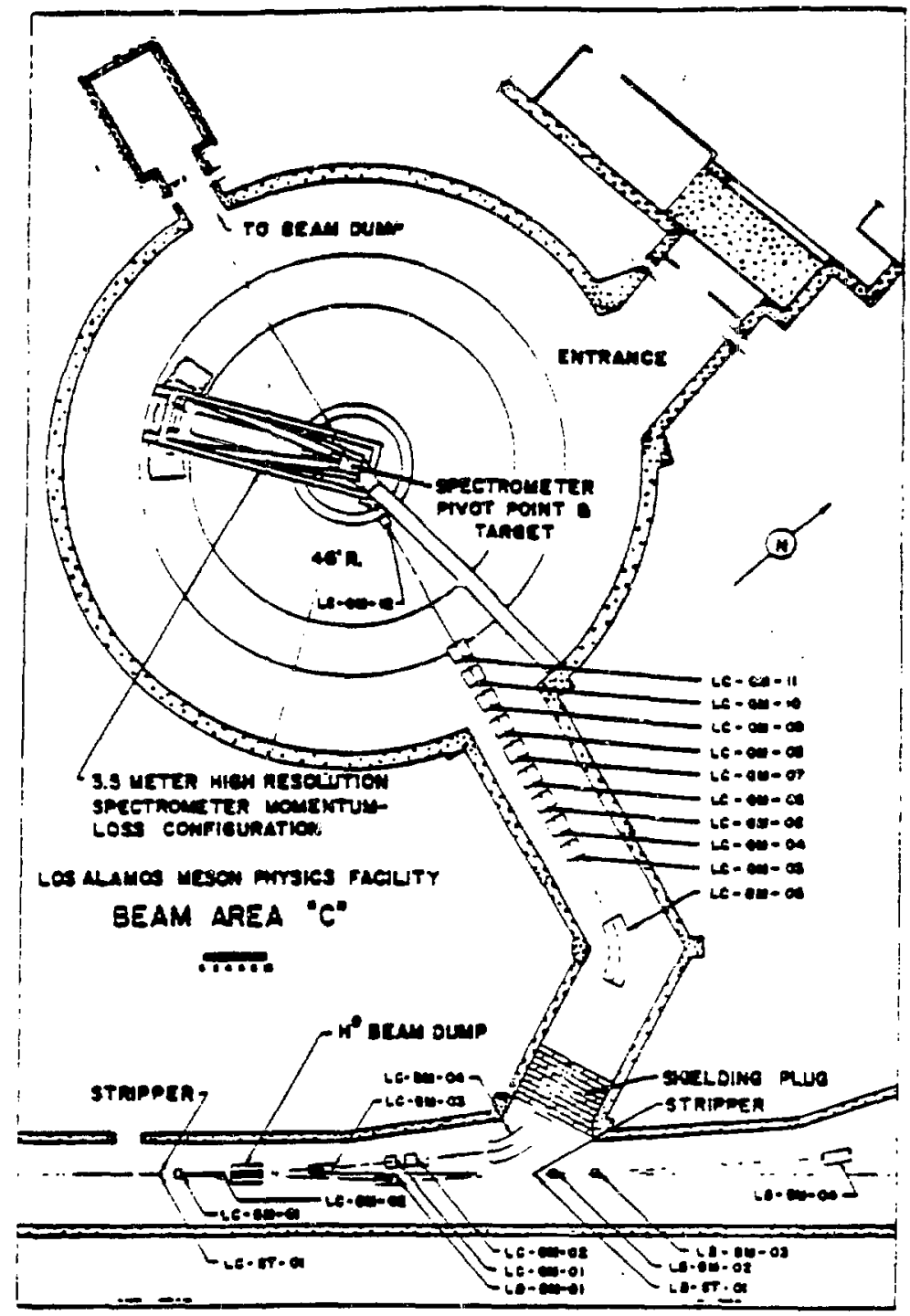

Eigure III-2. 
to several (about 16) nanoamperes at larger spectrometer angies ( > 12 degrees) where both lower background and reduced elastic scattering cross-sections permit increased beam currents. Large backgrounds at forward angles $1<12$ degrees) are caused by interactions of the unscattered beam with the magnet pole-faces of the spectrometer and the ait between the scattering chamber and the beam-dump tunnel. For angles greater than 12 degrees, the beam misses the spectrometer structure and an evacuated beam pipe may be inserted between the exit window of the scattering chamber and the beam-dump tunnel to reduce scattering from air.

\section{B. The High Resolution Spectrometer}

A section view of the HRs facility is shown in Figure III-3. The spectrometer consists of a quadrupoie and two dipoie magnets. We define a spectrometer co-ordinate system as foijows: let $z$ be in the beam monentum direction, let $x$ be perpendicular to $z$ and in the direction of the momentum dispersion of the beam, then $Y$ represents the transverse angular direction in such a way that $x, y$ and $z$ represent a right-handed system of axes.

The quadrupole magnet provides focussing in the $y$ direction and defocussing in the $x$-direction. Momentum analysis is accomplished by the dipoie magnets. The opticai properties of the system as a whole provide paraijei-topoint focussing in the $y$-direction and point-to-point 


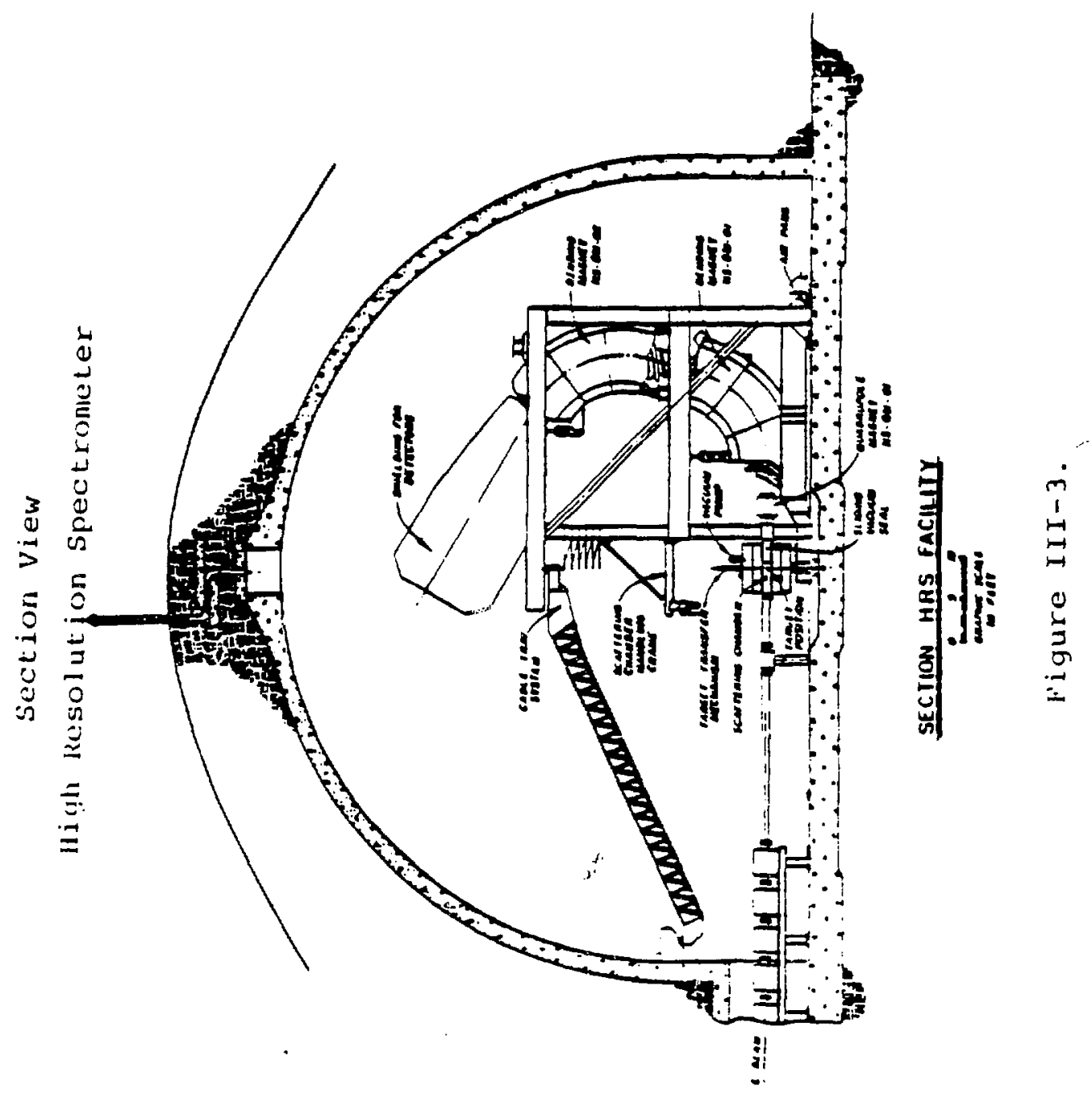


focussing in the $x$-direction, allowing precise angular and energy-loss measurement. Detalis of the operation of and beam transport through the spectrometer have been described previousiy ${ }^{38)}$.

\section{c. Targets}

Four targets were used in this work. These, togother with a varlety of other targets, were mounted on a wheel within the scattering chamber to Eacilitate target changing during the course of the experiments. The size of each aperture within which the targets were suspended was sufficiently large to essentialiy eliminate target frame- beam interactions.

The primary target was a natural spectroscoplc grade graphite strip of isotopic composition 98.9 i $^{12} \mathrm{C}$ and 1.18 ${ }^{13} \mathrm{C}$, and of thickness $44.7 \mathrm{mg} / \mathrm{cm}^{2}$. A $65 \mathrm{mg} / \mathrm{cm}^{2}{ }^{12} \mathrm{CH}_{2}$ foil was used for primary normailization purposes. Two ${ }^{208} \mathrm{~Pb}$ strip targets of thickness $19.8 \mathrm{mg} / \mathrm{cm}^{2}$ and $50 \mathrm{mg} / \mathrm{cm}^{2}$ were used for secondary normalization purposes, resolution checks and setup runs. Detalis of the measurement of the thickness of the graphite target will be discussed in Chapter IV.

of concern in choosing the thickness of the target for this work was the desire to optimize the experimental resolution. There are three basic components contributing to the observed resolution of 90 to $150 \mathrm{keV}$ FWaM for narrow states with intrinsle widths much less than this value. 
These are jenergy straggling induced by the target thickness and isotopic composition, a kinematic shift contribution $\frac{d T_{p}}{d \theta}$ which increases for larger scattering angles, and focussing abberations in the spectrometer.

Examination of a dotplot of Missing Mass as a Eunction of ${ }^{\theta}$ ip determined that the spectrometer focussing was optimized since no detectabie systematic correiations between these two variables could be seen. It is impossible to remove the kinematic broadening factor, and so the remaining adjustable parameter is the target thlckness.

Thicknesses of iess than $50 \mathrm{mg} / \mathrm{cm}^{2}$, while improving the resolution by reducing straggiing, did not provide sufficient count rates for rapid data acquisition. Targets with thickness greater than $50 \mathrm{mg} / \mathrm{cm}^{2}$ caused deterioration of the resoiution to some $200 \mathrm{keV}$ EWHM. It was therefore optimal in terms of both data rates and resolution to use a target of thickness about $50 \mathrm{mg} / \mathrm{cm}^{2}$. The overail experimentai resolution was then approximateiy $110 \mathrm{keV}$ FWHM.

\section{Beam Monttors}

The HRS facility is not yet equipped with a Faraday Cup for beam current monitoring. Reiative beam currents are monitored by two ionization chambers which can be placed in a position so as to intercept the unscattered beam within the scattering chamber after the target. 
The ionization chambers are gas-filled containers of rectangular construction with aluminized mylar windows of areal dimensions $3 \frac{1}{2} " \times 4 \frac{1}{2} "$ designed to accomodate dispersion of the beam by the target. Each chamber contains three parallel foils of aluminized mylar of thickness $0.25 \mathrm{mil}$. The outer foils are maintained at a potential of $-306 \mathrm{~V}$ relative to the central foil. The chambers are filled with a mixture of 908 Argon and $108 \mathrm{CO}_{2}$. One (ERø3) is maintained at an absolute pressure of $50 \mathrm{~mm} \mathrm{Hg}$ while the other (ER/4) is maintained at an absolute pressure of $200 \mathrm{~mm} \mathrm{Hg}$.

Ionization current generated by the passage of the energetic proton beam is collected at the central foil and passed through a LRS TTI-NIM converter and then into an ORTEC 439 current digitizer. The chambers are each linear to within 38 for beam currents ranging from $1 \mathrm{pA}$ to $10 \mathrm{nA}$, and relative yields monitored dureing the course of the experiments were constant to within $\emptyset .88$.

During acquisition of data at 3 degrees (laboratory) it was necessary to carefully adjust the location of the ionization chambers in order to minimize edge scattering from the chambers into the spectrometer acceptance. Neve theless, such edge scattering did result in high backgrounds in the first third of the spectrometer at this most fcrward angle setting. 
E. Focal PIane Detection System

Elastically and inelasticaliy scattered protons passing through the spectrometer are detected in an array of muiti-wire drift chambers and scintiliators mounted near the focal plane of the spectrometer. A dlagram of the detector system is shown in Figure III-4.

The scintiliators 52,53 and 54 provide puises which are used in the event trigger, and also energy- ioss and time- of- fight information for particle identification. The muiti- wire drift chambers DI and D2 are of the deiayine readout type deveioped at LAMPF by Atencio et. ai. ${ }^{40}$. Each chamber consists of two perpendicular sets of pianes, or four pianes each. Anode wires in each piane are of the aiternating gradient design with wire spacing of $8 \mathrm{~mm}$. Parailel pianes $C \times 1, C X 2$ for exampie, are offset by $4 \mathrm{~mm}$, or one half wire spacing. The anode wires, blased at $+2150 \mathrm{~V}$ with respect to the grounded cathode planes, are connected directiy into fast $(2.5 \mathrm{~ns} / \mathrm{cm})$ deiay ines which provide two anode outputs per plane. The active area of the chambers is $10 \mathrm{~cm}(y)$ by $60 \mathrm{~cm}(x)$.

The chambers are filied with a gas mixture $0=65$ Argon, 35 iso-butane and 0.108 Freon and operate at a pressure of $650 \mathrm{~mm} \mathrm{~kg}$. The anode signals are passed through 100 Ohm terminators into LRS LD604 fast ampifier-discriminators, converted to NIM fast ioglc and transmitted to the 
HRS Focal Plane Detector Array

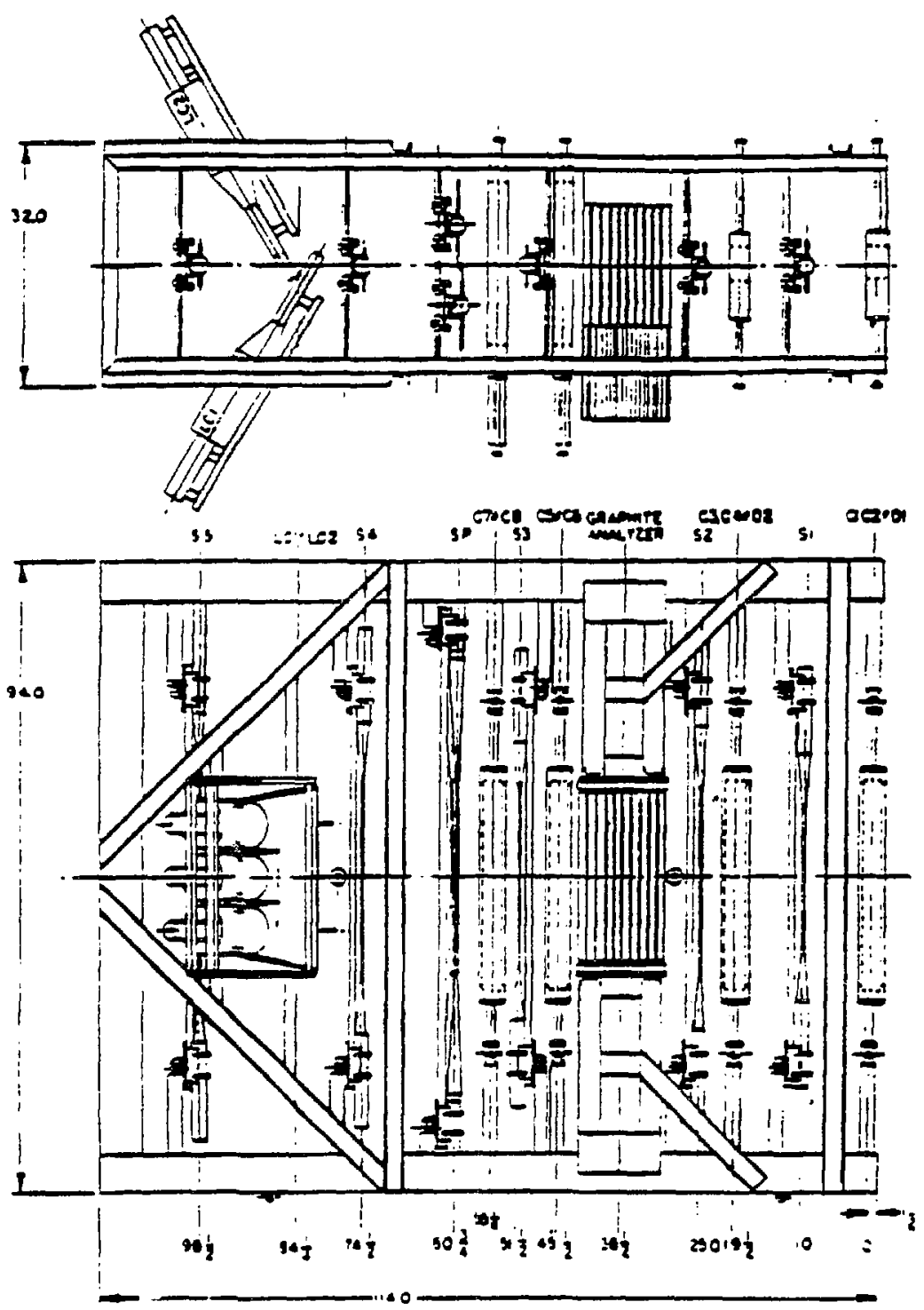

All dimensions in inches.

Figure III-4. 
uata acquisition area over some $200 \mathrm{~m}$ of 50 ohm coaxial cabie.

The chambers provide $x$ and $y$ position information for protons passing through the focal plane. From these data, the trajectory of the proton through the spectrometer may be reconstructed to determine scattering angle and missing mass information, for example.

$$
\text { E. Electronies and Data Acquisition }
$$

Raw data is acquired by a multi-ievel system consisting of (i) CAMAC crates housing ADC's (Anaiog to digitai converters), TDC's (Time to digital converters), Scalars and other devices; (ii) A MBD (Microprogrammabie branch driver) and (iii) a pdp $11 / 45$ computer with $124 \mathrm{k}$ of fast memory, dispiay terminais and a 1600 bpi Kennedy 9100 tape drive. A schematic cepresentation of this system is shown in Figure III -5 .

Fast NIM logic signais from the focal piane devices (wire chainbers and scintiliators) are regenerated in NIM ieading edge discriminators in the counting house. An event puise is generated using the fast signals from the coincidence $52^{\star} 53^{*} 54$. A Trigger puise is then generated by demanding the coincidence EVENT*RUN*BEAM* דUSY; 1.e. Event detected, run in progress, beam on and CAMAC not busy. Detaiis of the eiectronic module system used to generate the trigger puise, together with relevant timing information, 
are shown in Figure III-6. Thls Trigger puise issues a start signal to the TDC's and opens gates for ADC's, scalars and so on, while simultaneousiy triggering a 15 microsecond BUSY signal which disables the generation of new trigger puises for its duration. During this 15 microsecond interval the signals from the whe chambers and seintiliators are digitized and stored in a $9 \times 32$ bit storage buffer at each CAMAC address.

The 9 bit size provides a resolution of 512 channels per address, while the 32 bit dimension provides a buffered storage capacity for 32 digitized events at each CAMAC address. The compietion of event digitization and re-enabiing of the trigger generates a LAM (look-at-me) which is sent by the CAMAC crate to the MBD, and the MBD responds by reading the event from ai CAMAC addresses into a cireuiar buffer on a first-in, first-out basis. The MBD is then capabie of applying tests to various words of the event to determine whether the event should be kept for further processing or rejected. This process will be described in more detail in the next section. Suffice it to say that if the event falis the test the buffer does not rotate and the next event overwsites the event which falied the test. If the test was passed, the buffer cotates and the new event is read into an empty location. Events passing the MBD tests are designated as taped triggers.

The " $Q$ " data acquisition system 41 ) monitors the 
TO hRS fOCAL PLANE deTECTORS
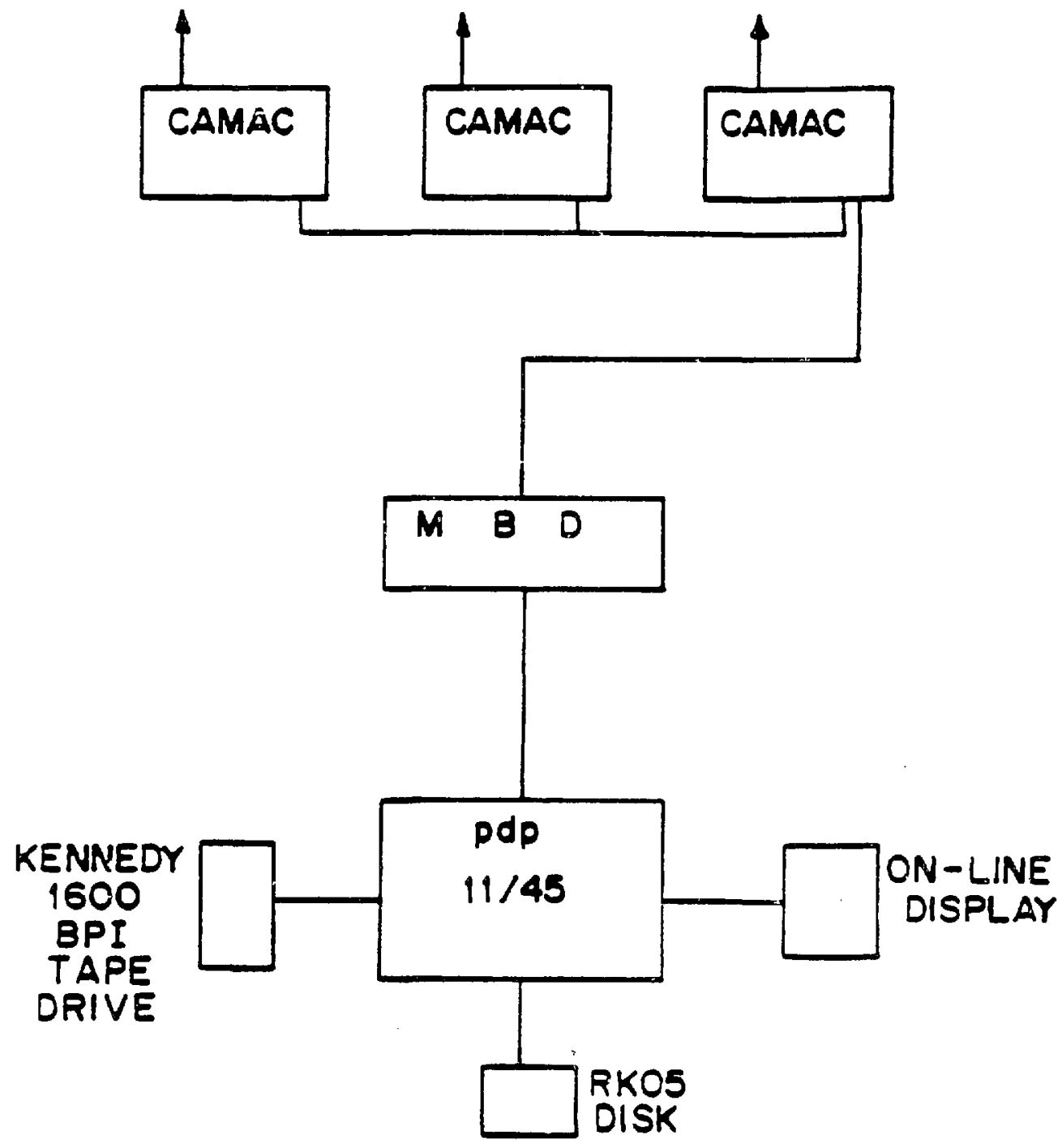

DATA ACQUISITION SYSTEM SCHEMATIC.

Figure III-5. 
operation of the MBD and reads every ten taped triggers into the memory of the pdp 11/45, whence they are transferred to tape. Depending upon the rate at whlch taped triggers are being read and written, time is avallable for some fraction of the events to pass through an event analyzer for on-Iine display. Typleally some 108 of the events were analyzed on-ilne.

The capability of the buffered CAMAC system to store 32 events per beam burst gives a maximum time-averaged data rate of 1280 events/sec, which is considerably superior to singie event transfer to the MBD.

\section{G. MBD Event Rejection}

The experiments described here were the first to utilize the capability of the MBD to selectiveiy reject events prior to processing for transfer to tape. The notivation for this is as foilows.

The $60 \mathrm{~cm}$ active area of the focal plane in the momentum dispersion direction $x$ corresponds to an excitation energy bite of some $18-30 \mathrm{MeV}$ depending upon the incident beam energy. The primary focus of the experiments was to study the $1^{+}, T=0,1$ states at $E_{X}=12.71$ and $15.11 \mathrm{MeV}$, and it was therefore desirable to place these states as ciose to the center of the focal plane as possible so as to optimize the resolution in this region. Consequently, states of jower excitation, such as the $2^{+}$state at $E_{x}=4.44$ Mev, 


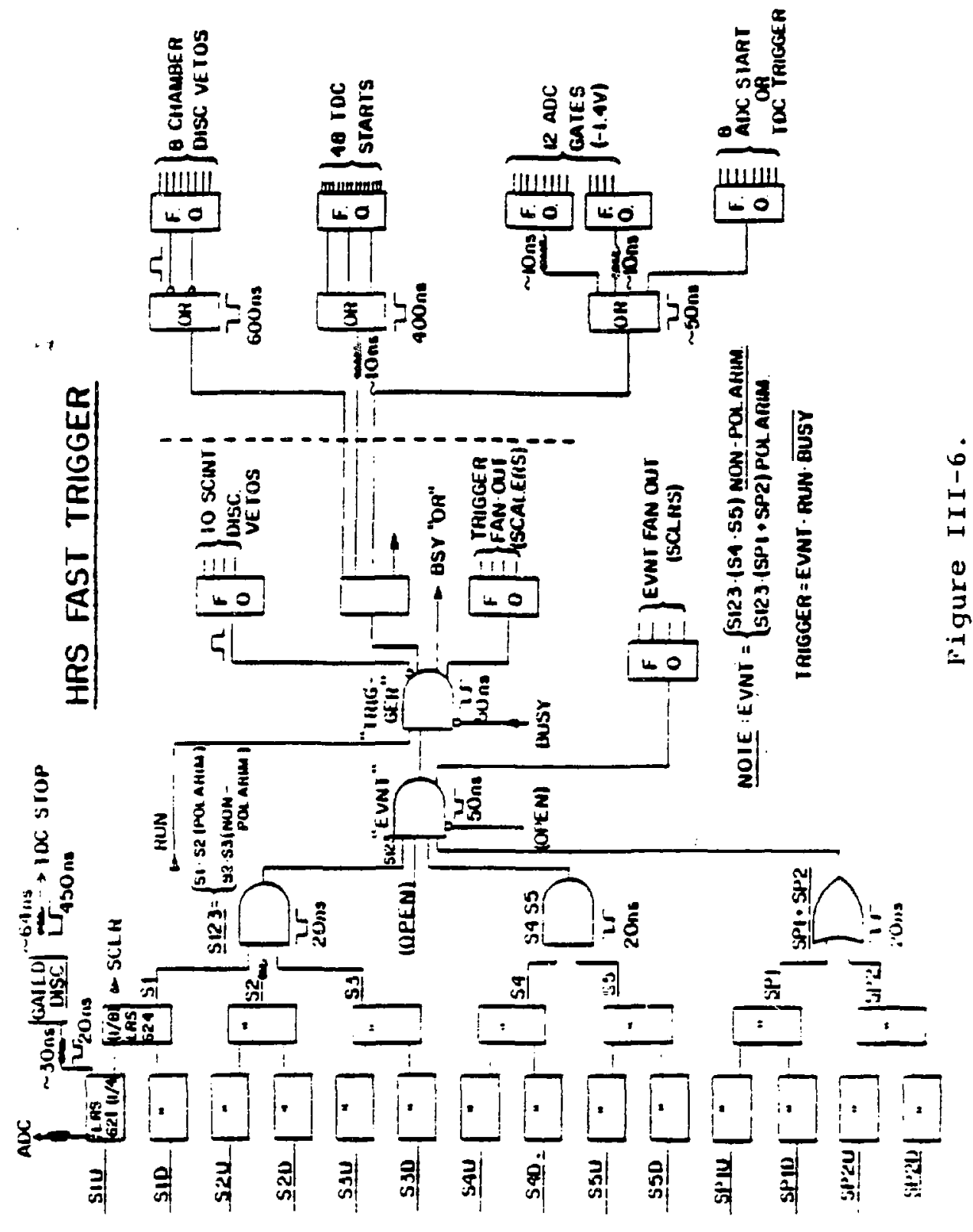


were constrained, to ile on the active area of the focal plane. At angles greater than 10 degrees, the natural parlty states such as the $2^{+}$dominate the spectrum and hence the yield of scattered particles. In addition, the peak-tobackground ratio in the region of interest becones smalier as the scattering angle increases. It was therefore desirabie to increase the efficlency of data taping of taped triggers in the region of interest.

The MBD was programmed to examine the raw $x$ position of the event in chamber 1 prior to processing. If the $x$ vaiue of the event feli outside of predetermined imits the event was fiagged for rejection. Not all such events were rejected. Depending upon the relative intensity of the states beling rejected it was possible to specify, together with the rejection limits, a certain fraction of events falIing outside those Ifmits which was to be retained. Typicaliy some 108 of the events in the rejection region wre retained for relative normalization purposes.

A sample MBD test program is given in the Append $1 x$. The cut I Imits were determined by examing a dotplot of Missing Mass vs $\operatorname{ClX}(\mathrm{N}-\mathrm{P})$ (Raw $x$ position in chamber 1). RecalIng that the $x$ - direction corresponds to the momentum dispersion direction of the spectrometer, the $x$ position is in first order related to Missing Mass, or excitation energy of the nucleus in the case of these experiments. The largest excltation energles are located at the smailest values 
of $x$ and vice versa. A typical dotplot of these variables exhibiting this phenomenon is shown in Figure III-7.

The dotplot was the used to determine the rejection Iimits on the variable $X I(N-P)$ as shown in Eigure III-7. These variables, together with the fraction of events to be retained were edited into the file MBDTST.DAT whlch loaded the tests into tlie MBD at the start of a run.

It is evident from the dotplot that a clean cut in $x$ is not a clean cut in Missing Mass, but rather introduces a cut of non-negigibie width in Missing Mass. A typicai spectrum of Missing Mass at $E_{p}=398 \mathrm{MeV}$ showing an MBD cut in the $E_{x}=10 \mathrm{MeV}$ region is shown in Elgure III-8. Typical cut widths in the Missing Mass spectrum were $700 \mathrm{keV}$ EuI width. Consequentiy some care had to be taken when extracting peak sums near these cuts to ensure that the cut was in no way interfering with the peak under consideration.

Unfortunateiy, piacing the cut at an excitation energy of about 10 Mev necessarily prevented measurement of complete angular distributions of the $1^{-} ; \theta$ state at $E_{x}=$ $10.84 \mathrm{MeV}$ For some beam energies. Nevertheiess, the gains in improved statistics per tape for unnatural parity states of interest cleariy outweigh this rather minimal ioss. 
Raw X Position (1) vs. Missing Mass

$234 *$ QEXACT * MISSING MASS

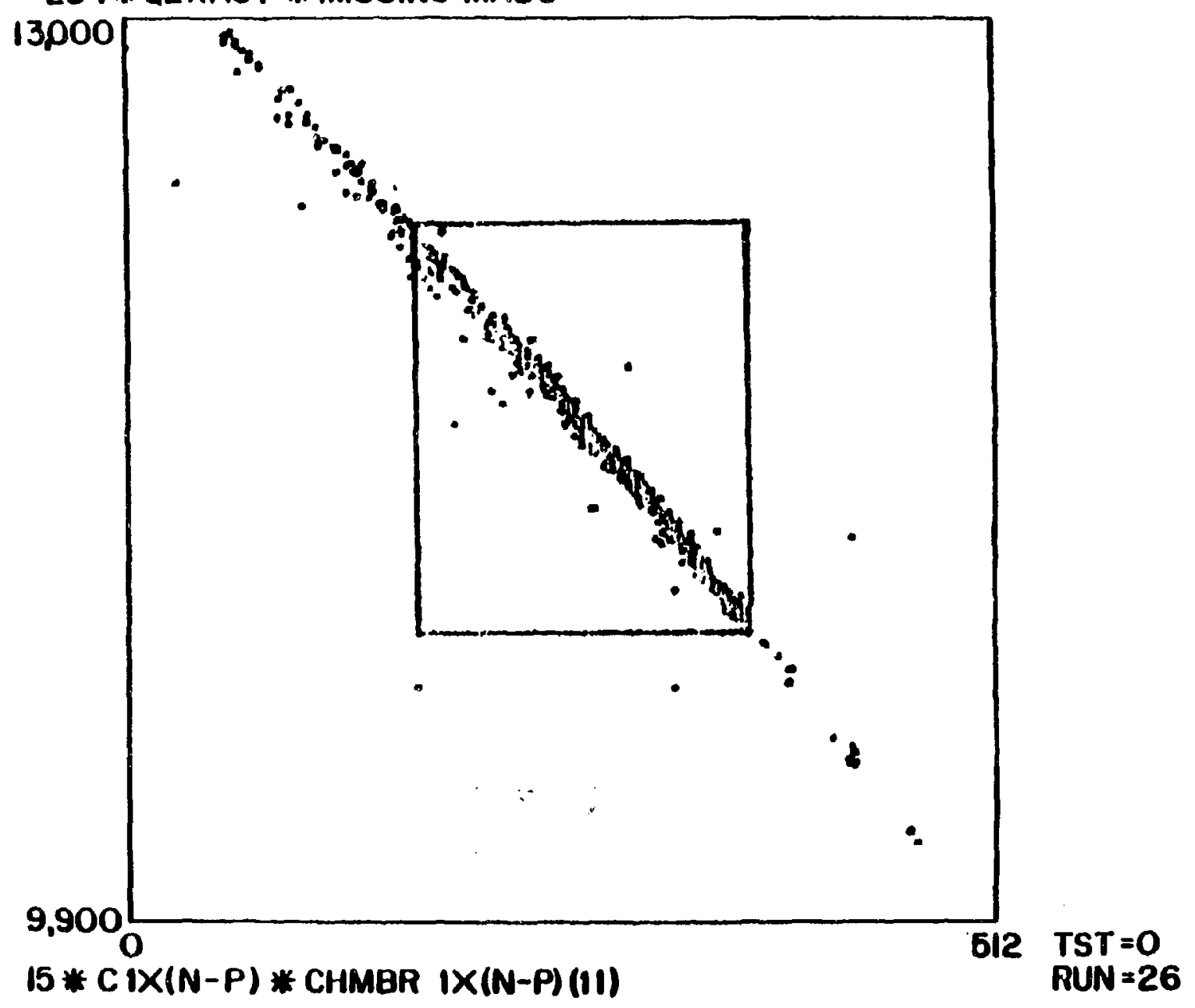

Figure III 7 . 


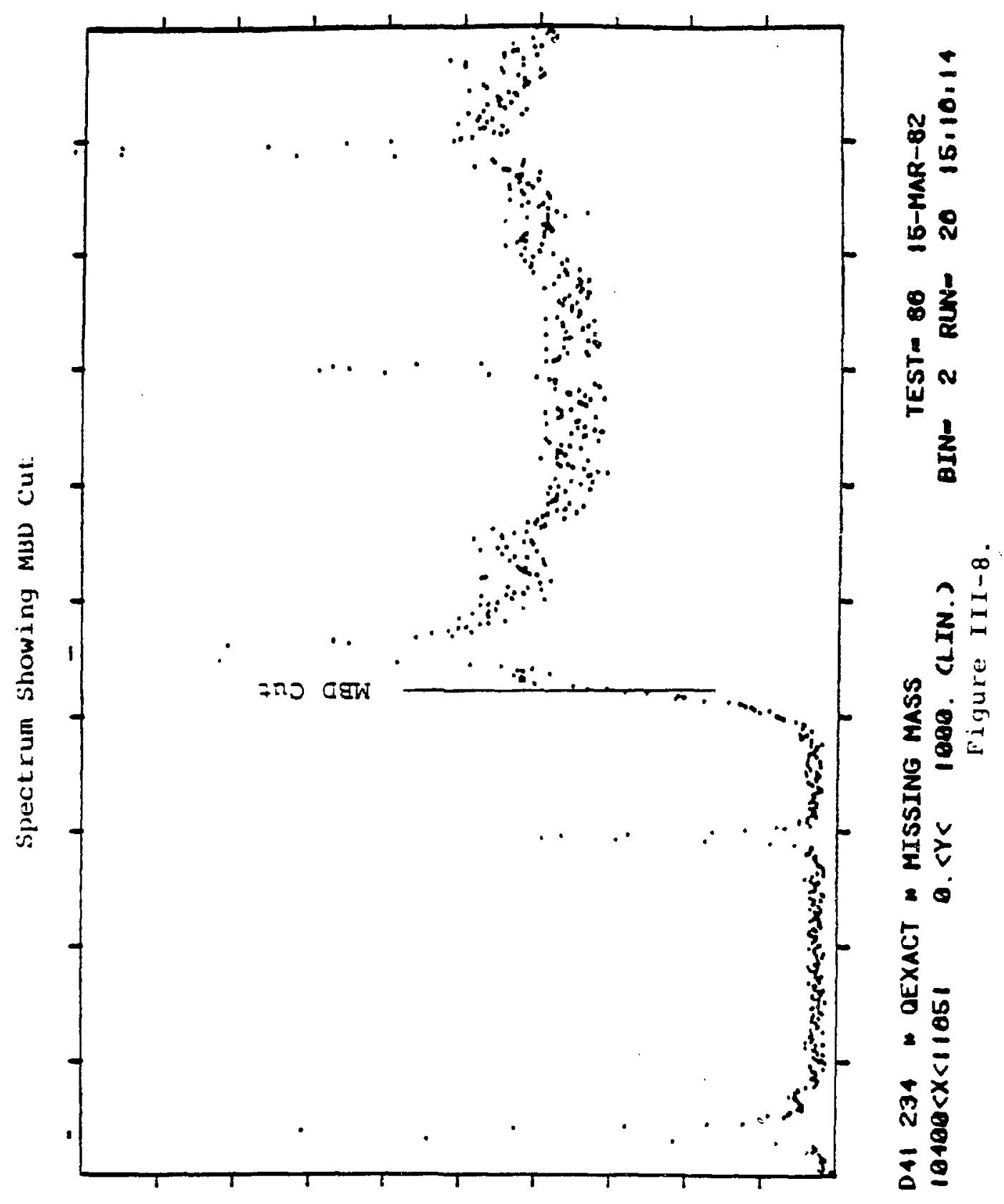




\section{DATA ANALYSIS AND REDUCTION}

\section{A) Event Processing and Testing}

Events successfuily passing the MBD tests outined previousiy and written to tape were analyzed both on- and off-Iine. The on-line analysis provided a rough estimate of yields during the running of the experiments, together with the means by which the satisfactory operation of the data acquisition system could be monltored. OnIy a small fraction (5-108) of the taped triggers were analyzed and histogrammed on-iine. Consequentiy the primary data reduction was performed off-ine on various computers at LAMPF.

Typicaily, events were read from tape into a pdp $11 / 60$ computer for processing by the anaiyzer HRSBUF $r$ unnlag under the data acquisition and analysis system Q.2. The anaiyzer appities a sequence of tests to seiected words associated with each event, the tests being specified in a user-defined file. An exampie of such a file used in the reduction of these data is given in the Appendix. Events successfuliy passing tests which specify a "good event" are then tested to determine into whlch histograms the analyzer should place them.

A total of 24 histograms were deflned in the replay of the data. Elghteen of the histograms were of missing mass and six were of true scattering angle gated on various states in the observed spectra. A sample THETASCAT histo- 
gram is shown in Figure IV-I. The lower and upper inits of the THETASCAT variable were determined from such a histogram gated on a weil-populated state in the spectrum, such as the eiastic scattering at forward angies. These bounds were used to determine the correct angular binning vaiues for THETASCAT in order to subdivide the spectrometer acceptance into two and three equal bins, and these vaiues were edited into the test flie. Events passing these tests could then be used to construct Missing Mass histograms for the various anguiar acceptance bins. The fuli acceptance, together with the two and three bin subdivisions provided six sets of missing mass histograms. Each set consisted of three separate histograms, one requiring beam poiarization 'normai', one requiring beam poiarization 'reverse' and one requiring 'normai or reverse', thereby creating the eighteen Missing Mass histograms.

The utility of subdividing the acceptance into varying degrees of fineness is evident from some of the more compiex spectra. Some states are very weakiy excited while others are more strongly excited. Those with sufficient statistics in the background-subtracted peak nay be extracted from the spectra subdivided into three bins without significant ioss of information, while those for which statistics are poor couid simuitaneousiy be extracted from the Euij acceptance spectra without necessitating a timeconsuming second repiay of the data. A sampie histogramming 
Spectometer Anqular Acceptance

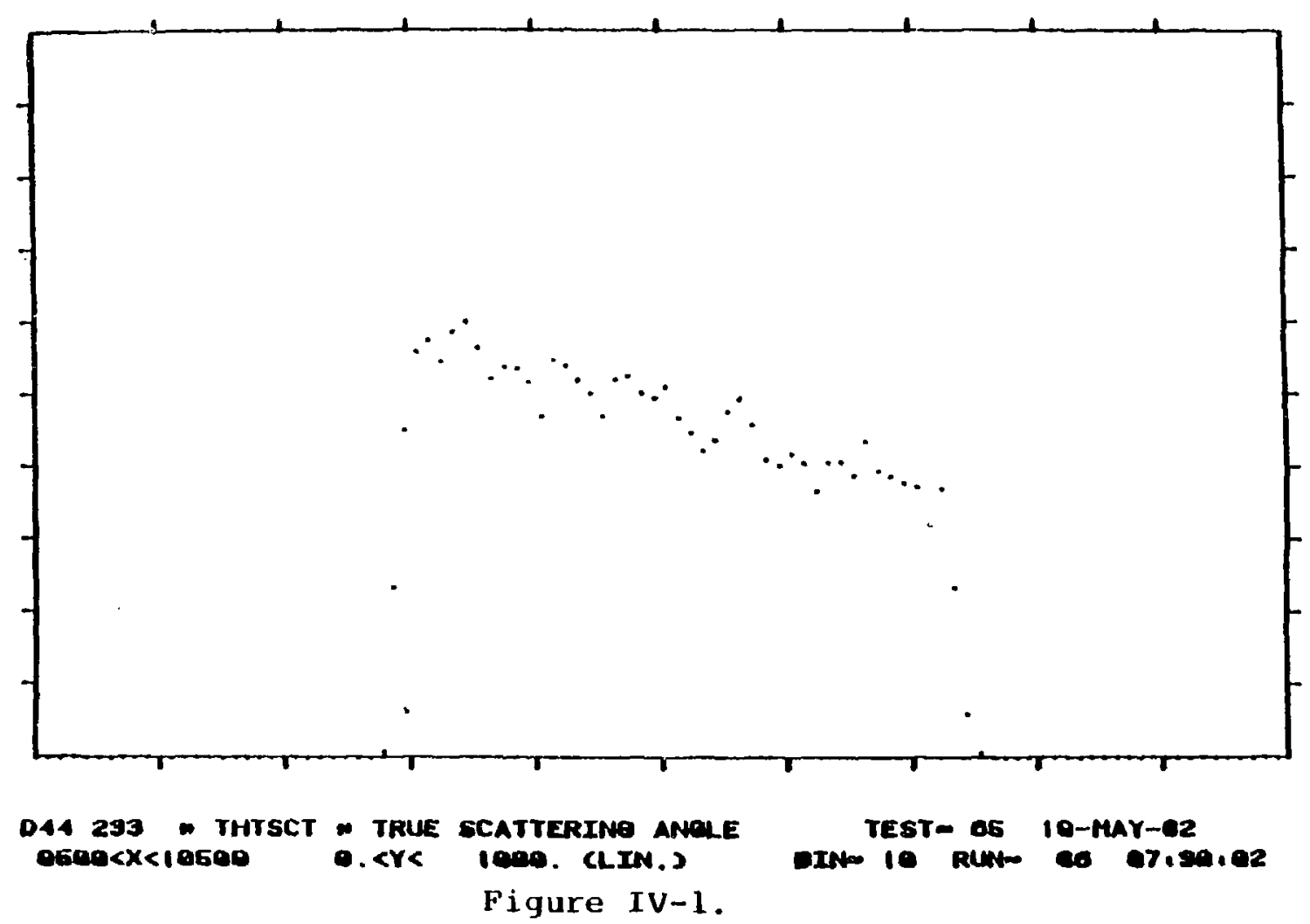


flie used in the data replay is given in the Appendix.

Histograms from varlous runs (tapes) at the same laboratory scattering angle were added together to form a final composite set of histograms useful for peak fitting. Associated with the final set of histograms was a test file ennumerating the numbers of events which had passed the varlous tests defined in the fije while the histograms were being generated, together with a scalar file containing the cumulative information recorded by the scaiars, such as digltized beam currents, normal and reverse beam gates and so on.

Three ciasses of histograms were finaily generated:

(a) A ciass comprising the excitation region 0.010.0 MeV, with and without MBD euts, for study of elastic scattering and excited states up to $E_{x}=9.64 \mathrm{MeV}$.

(b) A ciass comprising the excitation region 4.615.5 MeV, with and without MBD cuts, for study of ineiastic scattering in this excitation region.

(c) A class comprising the excitation region 12.025.0 MeV, with and without MBD cuts, for anaiysis of the excitation region 18.0-21.8 MeV.

The overlap states from class to ciass were used to provide relative normalization information. 


\section{B) Peak fitting}

Differential cross-sections and analyzing powers for the elastic scattering and the first excited state $\left(2^{+}, \theta ; E_{X}\right.$ $=4.44 \mathrm{MeV}$ ) were extracted from the program SHT applied to the replayed histograms. This program enables fine blinning of the angular acceptance for weli-populated, elean states with no apprectable background. As is evidenced by the spectrum shown in Figure IV-2 these two states clearly satisfy these criteria.

Yieids for all remaining observed states up to an excitation energy of $\mathrm{E}_{x}=20.6 \mathrm{MeV}$ were extracted at Rutgers using the line-ortented peak fitting program LOAF ${ }^{42)}$. This program enables backgrounds to be fitted with poiynomials of up to order 7, and peaks above such backgrounds to be fitted either to a reference peak shape defined in the spectrum or to a user-defined peak shape such as a gaussian. For narrow peaks (width approximately $100 \mathrm{keV}$, which approximately corresponds to the overall experimental resoiution) the peak for a given state in the Missing Mass (Normal or Reverse) Histogram was used as a reference peak for fitting subsequent Missing Mass Histograms in that run. For peaks of greater width, such as those in the $18-19 \mathrm{MeV}$ region, syntheticaliy generated gaussian peak shapes of the appropriate width were used.

States ldentified in the spectra are ilsted in Table 


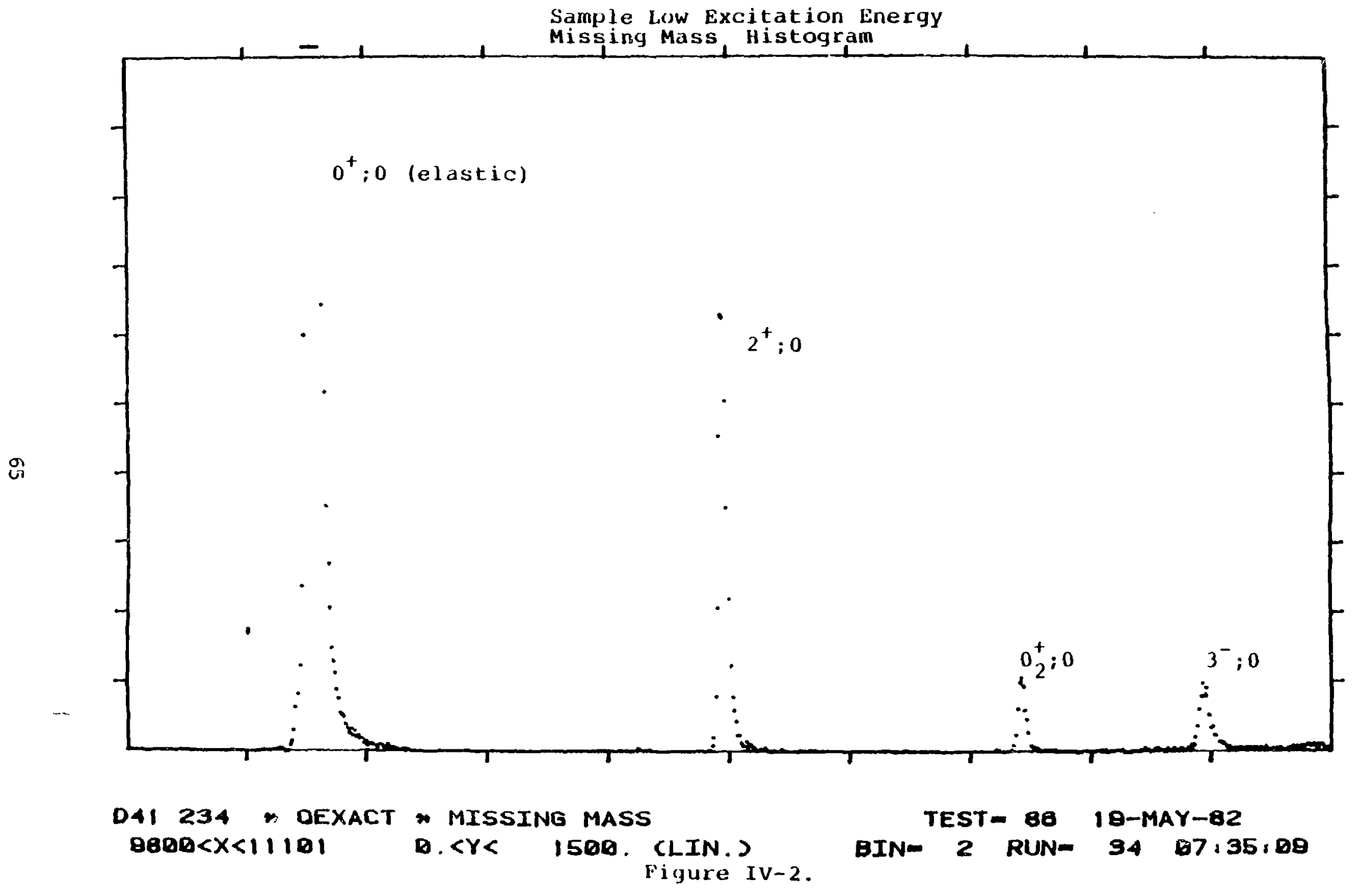


IV-l, together with observed widths where applicable. With the exception of the three states in the $19 \mathrm{MeV}$ complex, all are clearly resolved and weli-estabilshed. Identification and fitting of the three overiapping states in the $19 \mathrm{MeV}$ region was accomplished by a systemarle analysis of spectra at ali angles for all three energies.

For each energy it was found that this region was dominated by a single gaussian peak at 19.4 MeV for small vaiues of the momentum transfer $q$. As the value of $q$ increased a centroid shift to 19.65 heV togecher with a sight changed in width was observed. This was interpreted as follows. The state at $19.4 \mathrm{MeV}$ is dominant at low $\mathrm{q}$, while the state at $19.65 \mathrm{MeV}$ is dominant at high q, there being an intermediate region of $q$ where the diminishing $19.4 \mathrm{MeV}$ and rising $19.65 \mathrm{MeV}$ states are of comparable magnitude. In this intermediate reglon, peaks were fitted in a consistent way at both iocations. In the iarge q spectra it is quite evident that a third, broader state at $19.28 \mathrm{MeV}$ is present, and large $q$ flts were performed which inciuded this state. Widths and centrolds were fitted consistentiy from angle to angie and from beam energy to beam energy. Widths were primarily established by fitting the 1 solated peaks at low and high $q$ with varlous gaussian shapes, holding the centrold and background fixed. The widths tabulated weic taken to be those which optimized the chi-squared fitring procedure. In subsequent fits, especiaijy in overlapping regions, the 
Excitation energies and widths extracted from peak-fitting

in the $18-21 \mathrm{heV}$ region

\begin{tabular}{|c|c|}
$\Xi_{\mathrm{X}}(\mathrm{MeV})$ & $\Gamma(\mathrm{keV})$ \\
\hline $18.30=0.03$ & $380 \pm 30$ \\
$19.28 \pm 0.03$ & $580 \pm 50$ \\
$19.40 \pm 0.02$ & $480 \pm 40$ \\
$19.65 \pm 0.03$ & $440 \pm 40$ \\
$20.60 \pm 0.02$ & $280 \pm 20$ \\
\hline
\end{tabular}

Table IV-1. 
widths were held constant, as were the centrolds. The errors in the centroid locations were determined by fixing the width and allowing the centrold of the peak to vary during a peak-fitting minimization on the isoiated peak. Simiar tests were performed on overlapping peaks and resuited in no significant increase in the assigned uncertainties in peak location. Sample fits for this region are shown in Figures $I V-3$ and $I V-4$

On the basis of the procedure outined above, it was concludad that no fewer than three states could be clearly Identifled in the $19 \mathrm{MeV}$ region. It is conceivabie that the state at $19.55 \mathrm{MeV}$ could be a composite of two or more closeiy overlapping states. However, since consistent fits could be made with a singie gaussian shape there was no basis upon which such a decomposition couid be made, and we therefure treat the peak obser.ed at $19.65 \mathrm{MeV}$ as a single state in subsequent anaiysis.

C) Differentiai Cross-sections and Absoiute Normaiization

Differential cross-sections and anaiyzing powers were computed from the yields obtalned from peak fitting by use of the progran APOWR. Appropriate corrections for beam poiarization, chamber efficiency, live rimes and so on are made by the program through data obtained from the scalar and test resuit output fron the analysis program. The most 
low y lligh lixcitation linergy Spectrum

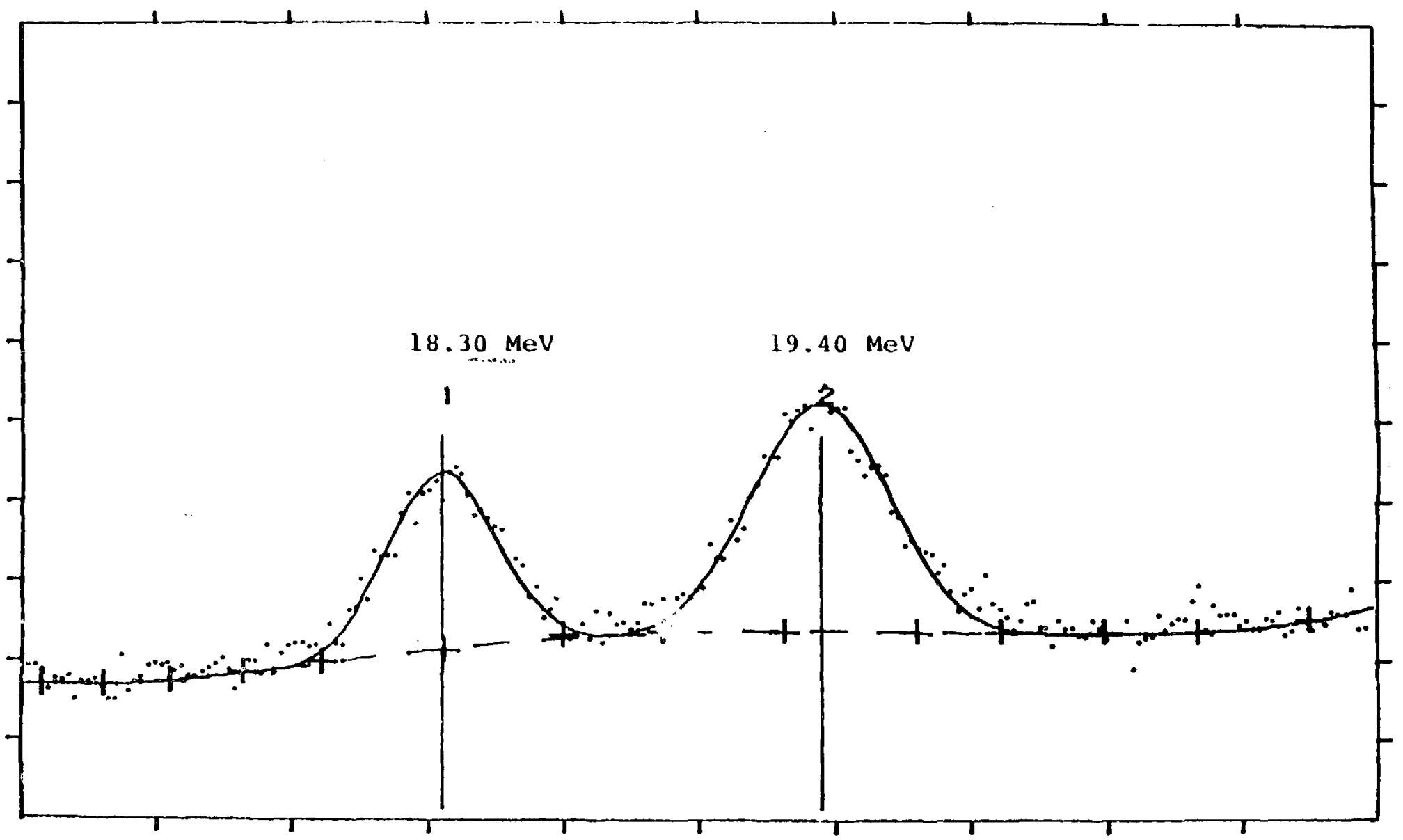

D41 234 DEXACT * MISSING MASS

TESTE 72 25-JUL-82 $11700<x<12105$

D. $<Y<\quad 1000$. CLIN.) BIN= 2 RUN= 11200.24 .53 
High q lligh Excitation Energy Spectrum

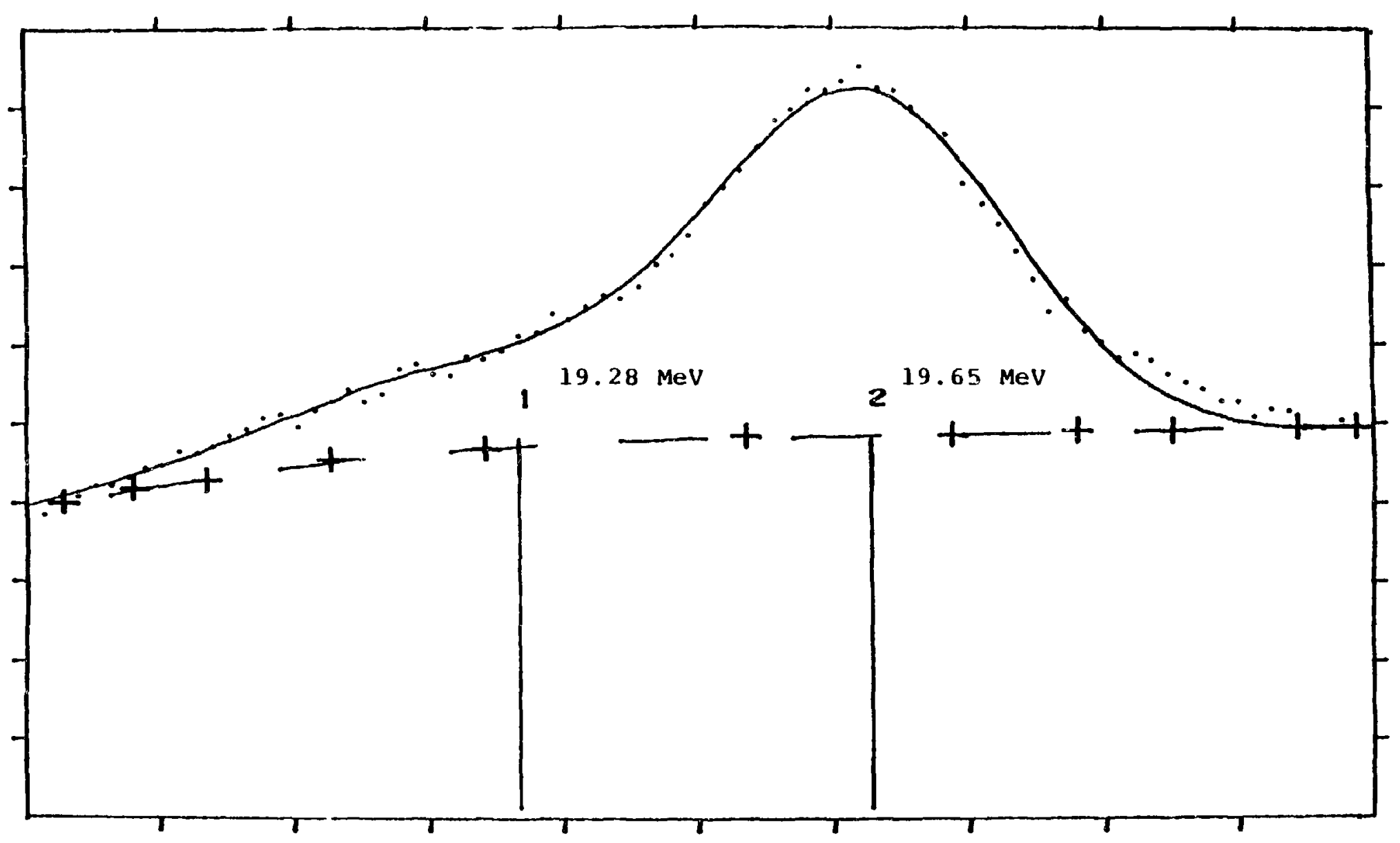

D4 234 " OEXACT " MISSING MASS

TESTR 72 25-JUL-62

D. KY< 6000. CLIN.J Figure IV-4. 
Witical factor affecting the differential cross-section in these computations was that used to convert the reiative corss-sections into absoiute units.

The cross-section is computed using the formuia

$$
\frac{d \sigma}{d \Omega}=N F * G *\left[\frac{\frac{Y_{N} * C F N}{E R \phi 3 N} * P N-\frac{Y_{R} * C F R}{E R \emptyset 3 R} * P R}{P N-P R}\right] \quad I V-1
$$

where $Y_{N(R)}$ is the normal (reverse) polarization yeild, $C F N(R)$ is the normal (reverse) polarization correction factor, ERGiN(R) is the normal (reverse) poiarization beam current monitor digitized value, $P N(R)$ is the normai (reverse) beam poiarization, $G$ is the Jacobean for transformation frow iaboratory to center-of-mass co-ordinates and NE is an absoiute normalization factor which is dependent on beam energy.

The anaiyzing power is computed using the formuia

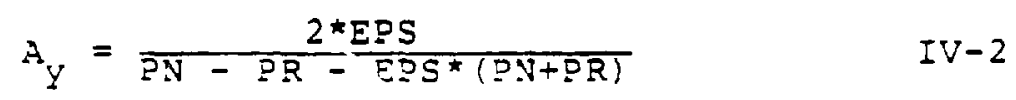

where

$$
E P S=\frac{Y_{N}-Y_{R}}{Y_{N}+Y_{R}}
$$

If $P N=-P R=P_{B}$, then this expression reduces to the EamiIiar

$$
A_{Y}=\frac{1}{P_{B}}\left[\frac{Y_{N}-Y_{R}}{Y_{N}+Y_{R}}\right]
$$

Since PN $\neq-P R$ in most zases, the additionai term in the denominator adjusts for this effect so that $A_{Y}$ majbe computed correctiy. 
In general, all parameters used in the above formuiae have uncertainties associated with them. Uncertainties in PN(R) are determined by statistical means from counts accunuiated in the line $C$ polarimeter. Uncertainties in beam current monitoring have been discussed previously, and resuit in uncertainties in the vaiues of the $\operatorname{ERg} \mathrm{N}(\mathrm{R})$ scaiars of about 38. The correction factors are determined by scalar counts that are typicaliy of the order of $10^{5}$ or greater. Assuming the errors in these numbers to be pureiy statistical, the uncertainty in the correction factors is iess than 0.18 and may be negiected.

Uncertainties for $P N(R)$ and $E R G 3 N(R)$ are inciuded in the computation of errors in the relativedifferential cross-sections and aiso the anaiyzing powers, as are the uncertainties in the yields $Y_{N(R)}$. The uncertainties in the yieids are twofold: there are statisticai uncertainties in the peaksums, and aiso, for states for which the peak-tobackground ratio is small there are associated background subtraction exrors. These two sources of uncertainty nave been combined where applicabie to provide conservative estimates Eor uncertainties in the measured yieids, and, in gens erai, the uncertainties in the yieids dominate the computed uncertainties in the relative differentiai cross-sections and the anaiyzing powers.

At the time this work was performed, iack of accus rately calfbrated beam monitors at the HRs preciuded abso- 
Iute normalization of the data by measurement of the incident beam charge. Since these experiments were among the first conducted at beam energles beiow 800 MeV at LAMPF, few data exist for comparative purposes. Although some eiastic and inelastic proton scattering differential cross-section data from ${ }^{12} \mathrm{C}$ exlst at incident beam energies of 402 and 600 Mev'3, 14), it would not be wise to normalize to these data because the results of the current work shouid be viewed as compiementary to such existing data.

Absoiute normaization was accomplished by utilizing availabie nucieon-nucieon eiastic scattering data. At each incident beam energy the yield for the scattering process $I_{H}(p, P)^{l_{H}}$ was measured. Comparison with tabuiated data and phase shift caicuiations yieided a normailzation factor which couid then be appiled to the ${ }^{12} \mathrm{c}$ data.

The graphite target used for data acquisition was weighed and the ${ }^{12} \mathrm{C}$ thickness was determined to be $44.7 \pm$ 1. $0 \mathrm{mg} / \mathrm{cm}^{2}$. The uncertainty resuits from the deposit of smail amounts of glue on the upper and iover edges from the mounting syseem used to suspend the foij in the target frame. The glue was not in the region of the target intercepted by the proton beam during the experiments. The Euii foil ${ }^{2}{ }^{2} \mathrm{CH}_{2}$ target used for normaiization purposes was found to have a thickness of $65.0 \pm 0.5 \mathrm{mg} / \mathrm{cm}^{2}$. The carbon content of this target is then $55.7 \pm 0.43 \mathrm{mg} / \mathrm{cm}^{2}$, while the hydrogen content is $9.3 \pm 0.07 \mathrm{mg} / \mathrm{cm}^{2}$. 
The ratio of carbon Thickness for the two targets was verified at a single angle for each beam energy by measuring the yield of elastically scattered protons from the ${ }^{12} \mathrm{C}$ nuclei. Results are given in Table IV-2. The ratios so measured were self-consistent to within 28 , but showed a mean systematic deviation of some $4 \%$ from the value obtained by weighing. The fact that the mean measured ratio reflects a slightly smaller thickness may be understood in terms of the glue deposits visible on the target and discussed previously. In subsequent discussions a mean ${ }^{12} \mathrm{c}$ target thickness for the graphite target of $43.7 \pm 1.0$ $\mathrm{mg} / \mathrm{cm}^{2}$ will be used.

For a given arbitrary normalization factor NF, let the relative APOWR differential cross sections for ${ }^{1} H(p, p){ }_{H}$ be $\sigma_{A P}$ and for ${ }^{12} C(p, p){ }^{12} \mathrm{C}$ be $\sigma_{A C}$. If an absolute differential cross section for ${ }^{1} H(p, p)^{1} H, \sigma_{p^{\prime}}$ is known at a given angle $\theta_{n}$, then absolute normalization of the measured carbon differential cross sections may be accomplished by computing

$$
\sigma_{C}(\theta)=\sigma_{A C} * \frac{p}{N_{C}}\left[\frac{\sigma_{p}\left(\theta_{n}\right)}{\sigma_{A P}\left(\theta_{n}\right)}\right] \quad \quad I V-5
$$

where the factor $\mathrm{N}_{p} / \mathrm{N}_{c}$ expresses the ratio of the number of $1_{\mathrm{H}}$ nuclei per unit area to the number of ${ }^{12} \mathrm{C}$ nuclei per unit area in the $\mathrm{CH}_{2}$ and graphite targets respectively.

The quantity $\mathrm{N}_{\mathrm{p}} / \mathrm{N}_{\mathrm{c}}$ may be written as

$$
\frac{\mathrm{N}_{\mathrm{p}}}{\mathrm{N}_{\mathrm{C}}}=\frac{{ }^{2 \mathrm{M}_{\mathrm{CH}}}{ }^{\mathrm{T}} \mathrm{C}}{{ }_{\mathrm{C}}{ }^{\mathrm{T}} \mathrm{CH}_{2}}
$$


where $T$ is the thickness of the appropriate target in $\mathrm{mg} / \mathrm{cm}^{2}$, and $\mathrm{M}_{\mathrm{c}},{ }^{\mathrm{M}_{\mathrm{CH}}}$ are the atomic and molecular masses of ${ }^{12} \mathrm{C}$ and ${ }^{12} \mathrm{CH}_{2}$ respectiveiy. This factor is found to be 2.54 \pm 0.06 .

The measured reiative differential cross-sections and corresponding absolute cross-sections $\sigma_{A P}$ and $\sigma_{p}$ are given in Tabie IV-3. Errors in the rejative cross-sections are pureiy statisticai, there being minimai background under the eiastic scattering peaks. A possibie source of uncertainty in the hydrogen scattering peak sums is as foilows. The incident protons can exclte the carbon nuclei in the cH2 target. Aithough the dipole and quadrupole fieids of the spectrometer are set to focur protons elasticaily scattered from ${ }^{1} \mathrm{H}$ nuciei in the $\mathrm{CH}_{2}$ foll, the energy ioss for protons ieaving the ${ }^{12} \mathrm{C}$ nucieus in the $9.64 \mathrm{MeV}$ excited state $1 \mathrm{~s}$ so as to generate outgoing protons with the same momentum as those scattered from the ${ }^{H}$ nuciei. However, because of kinematic differences in the scattering from ${ }^{12} \mathrm{C}$ and ${ }^{1} \mathrm{H}$, these protons are not optimaiiy focussed on the focai piane of the spectrometer, and are skewed as shown in Eigure IV1. It may be seen that it is possibie for some of the particies inelasticaily scattered from the carbon nuciei to contribute to the ${ }^{1} \mathrm{H}$ peak sum. However, at the iaboratory angies at which the normaization data were taken, the excitation of the $9.64 \mathrm{MeV}$ state was so weak as to make no signiflcant systematic contribution to the sources of error in 
Target Weight Ratios

\begin{tabular}{|c|c|c|c|}
\hline $\mathrm{E}_{\mathrm{p}}(\mathrm{MeV})$ & ${ }^{\theta} \mathrm{cm}$ (deg) & $\frac{T_{c}(C)}{T_{c}\left(C_{2}\right)}$ & $\begin{array}{l}\frac{\mathrm{T}_{\mathrm{G}}(\mathrm{C})}{\mathrm{T}_{\mathrm{C}}\left(\mathrm{CH}_{2}\right)} \\
\text { Weighing }\end{array}$ \\
\hline 398 & 12.84 & 0.754 & 0.803 \\
\hline 597 & 14.90 & 0.769 & 0.803 \\
\hline 698 & 13.73 & 0.774 & 0.803 \\
\hline
\end{tabular}

Table IV-2.

Pelative and Ajsolute Cross-sections and Normalization Factors by $B$ in

\begin{tabular}{|c|c|c|c|c|c|c|c|}
\hline$E_{p} \quad(M \in V)$ & ${ }^{\theta} \mathrm{cm}$ (deg) & $\begin{array}{l}\sigma_{\AA P} \\
\text { (rel. }\end{array}$ & $\begin{array}{l}x 10^{4} \\
\text { units) }\end{array}$ & $\sigma_{P}$ & $\left(\frac{m b}{s I}\right)$ & $\frac{\mathrm{N}_{p}}{\mathrm{~N}_{c}} * \frac{\sigma_{P}}{\sigma_{A P}}$ & $\left(\frac{m b}{s r}\right)$ \\
\hline 398 & 10.83 & $6.0 .28=$ & \pm 0.265 & 4.58 & \pm 0.07 & $1.94=$ & $=0.10$ \\
\hline 398 & 12.08 & $5.829=$ & \pm 0.268 & 4.43 & \pm 0.07 & $1.93=$ & $=0.11$ \\
\hline 398 & 13.33 & 5.494 & \pm 0.265 & 4.42 & \pm 0.07 & $2.05=$ & $=0.12$ \\
\hline 398 & $18 . \overline{-62}$ & $5.961=$ & \pm 0.189 & 4.25 & \pm 0.15 & $1.81 \pm$ & $=0.10$ \\
\hline 398 & 19.85 & $5.880=$ & \pm 0.212 & 4.24 & \pm 0.15 & $1.83 \pm$ & $=0.10$ \\
\hline 597 & 13.57 & $9.005=$ & \pm 0.325 & 7.52 & \pm 0.90 & $2.12 \pm$ & $=0.27$ \\
\hline 597 & 14.87 & $9.113=$ & \pm 0.336 & 7.56 & \pm 0.90 & $2.11=$ & $=0.27$ \\
\hline 597 & 16.17 & $8.633=$ & \pm 0.325 & 7.49 & \pm 0.90 & $2.21=$ & $=0.28$ \\
\hline 698 & 12.40 & $11.46 \pm$ & \pm 0.409 & 11.0 & \pm 1.7 & $2.44 \pm$ & $=0.39$ \\
\hline 698 & 13.73 & $14.28=$ & \pm 0.444 & 10.6 & \pm 1.7 & $1.89 \pm$ & 0.31 \\
\hline 698 & 15.06 & $14.35=$ & $=0.457$ & 10.5 & $=1 . .7$ & $1.86 \pm$ & 0.31 \\
\hline
\end{tabular}

Table IV-3. 
the normalization.

Absolute ${ }^{1} H(p, p) 1_{H}$ differential cross-sections were determined from tabulated data and phase shift calcuiations from a phase shtet analysis program provided by Arndt 32 ) and references therein. At $398 \mathrm{MeV}$, agreement between such calculations and available data $1 \mathrm{~s}$ good. The situation was found to be iess clear at 597 and $698 \mathrm{MeV}$, where data are not avaiiabie at the exact energy or angie, and discrepancies between caicuiation and data are observed. The value of ${ }_{p}$ at $597 \mathrm{MeV}$ was determined by inear interpolation between data at the same momentum transfer at 572 and 648 Mev. The resuiting interpoiated vaiue was then averaged with a mean caicuiated vaiue from different phase shift soiutions at $597 \mathrm{MeV}$. The error quoted contains contributions from experimentai uncertainty, computational uncertainty and interpoiation uncertainty.

At $698 \mathrm{MeV}$ the value for zo was determined by averaging various phase shift soiutions, since no data were avaliabie at the angie in questiun. Eor consistency, piase shfet soiutions used were compared with other avaijabie data at the same energy not too far from the normaiization angie, and agreement was found to be reasonabie. The iarge uncertainty in to is due mainiy to averaging procedures and deviations of caicuiated soiutions from measured vaiues.

The normaitization factors $\quad V F=\frac{N}{N} \frac{J}{J_{P}}$ 
determined using the procedures described above are given in Table IV-4. Uncertainties are the result of the application of standard error propagation techniques, and range from 78 to 218. These factors have been applied to all relative ${ }^{12} \mathrm{C}$ differential cross-sections to yield absolute differential cross-sections.

Differential cross-sections and anaiyzing powers for ail states extracted at each beam energy are tabuiated in the Appendix. 
Absolute Normalization Factors

\begin{tabular}{|l|l|}
$E_{p}(\mathrm{MeV})$ & $\overrightarrow{\mathrm{N}}_{\mathrm{p}} * \frac{\sigma_{p}}{\sigma_{\mathrm{AP}}} \times 10^{-8}\left(\frac{\mathrm{mb}}{\mathrm{sr}}\right)$ \\
\hline 398 & $1.912 \pm 0.134$ \\
597 & $2.147 \pm 0.277$ \\
698 & $2.063 \pm 0.431$ \\
\hline
\end{tabular}

Table IV-i. 


\section{THE DATA}

In this chapter we present and discuss the nain qualitative features of the data. General observations concerning raw spectra are made, followed by a more detaijed discussion of observed differential cross sections and anaiyzing powers.

\section{A) Spectra}

A Iarge number of spectra covering different regions of excltation energy were obtained at each beam energy. The crlteria for the construction of these spectra have already been presented. The qualitative features of the spectra are relativeiy independent of incident beam energy, so we choose to present and discuss a seiection of spectra taken at a beam energy of $398 \mathrm{MeV}$. These spectra are presented in F1gures $V-I$ to $V-3$. Each of these figures consists of three spectra taken at representative vaiues of the momentum transfer varlabie $q\left(0.34,0.97\right.$ and $\left.1.75 \mathrm{Em}^{-1}\right)$ for a glven range of excitation energy.

Elgure $V-I$ shows spectra for an excitation region of 0.0 to $11.0 \mathrm{MeV}$. The spectrum for $q=0.34 \mathrm{fm}^{-1}$ is dominated by elastic scattering, with littie or no strength seen in the $2_{1}^{+}\left(E_{x}=4.44 \mathrm{MeV}\right), \theta_{2}^{+}\left(E_{x}=7.65 \mathrm{MeV}\right)$ or $3_{1}^{-}\left(E_{x}=9.64\right.$ Mevl states. The spectrum at $q=0.97 \mathrm{fm}^{-1}$ shows the continued dominance of the eiastic peak, but the $2_{1}^{+}, 0_{2}^{+}$and $3{ }_{1}^{-}$ states are now also strongly exclted. Note also that back- 
Low Excitation Spectra

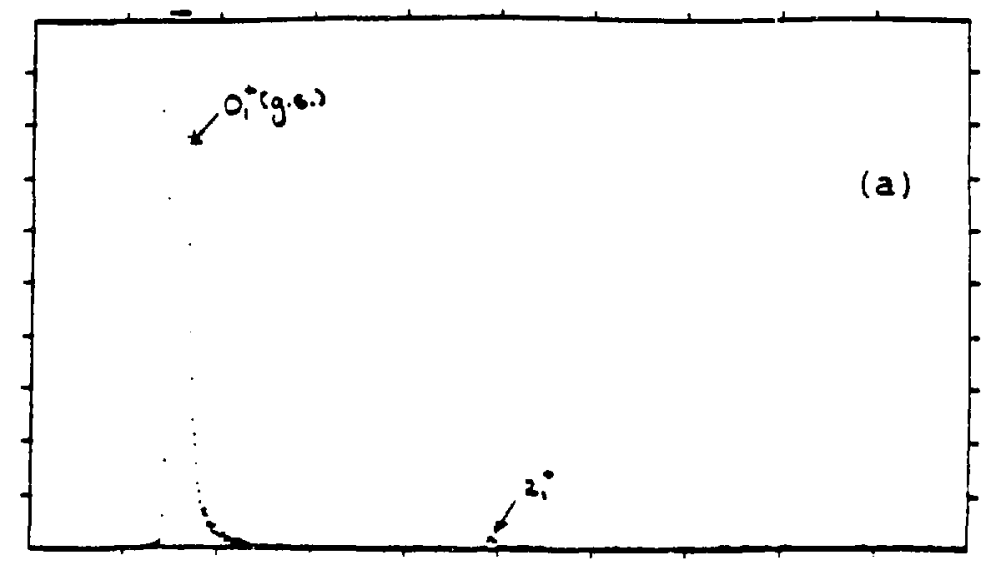

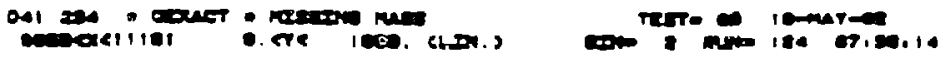
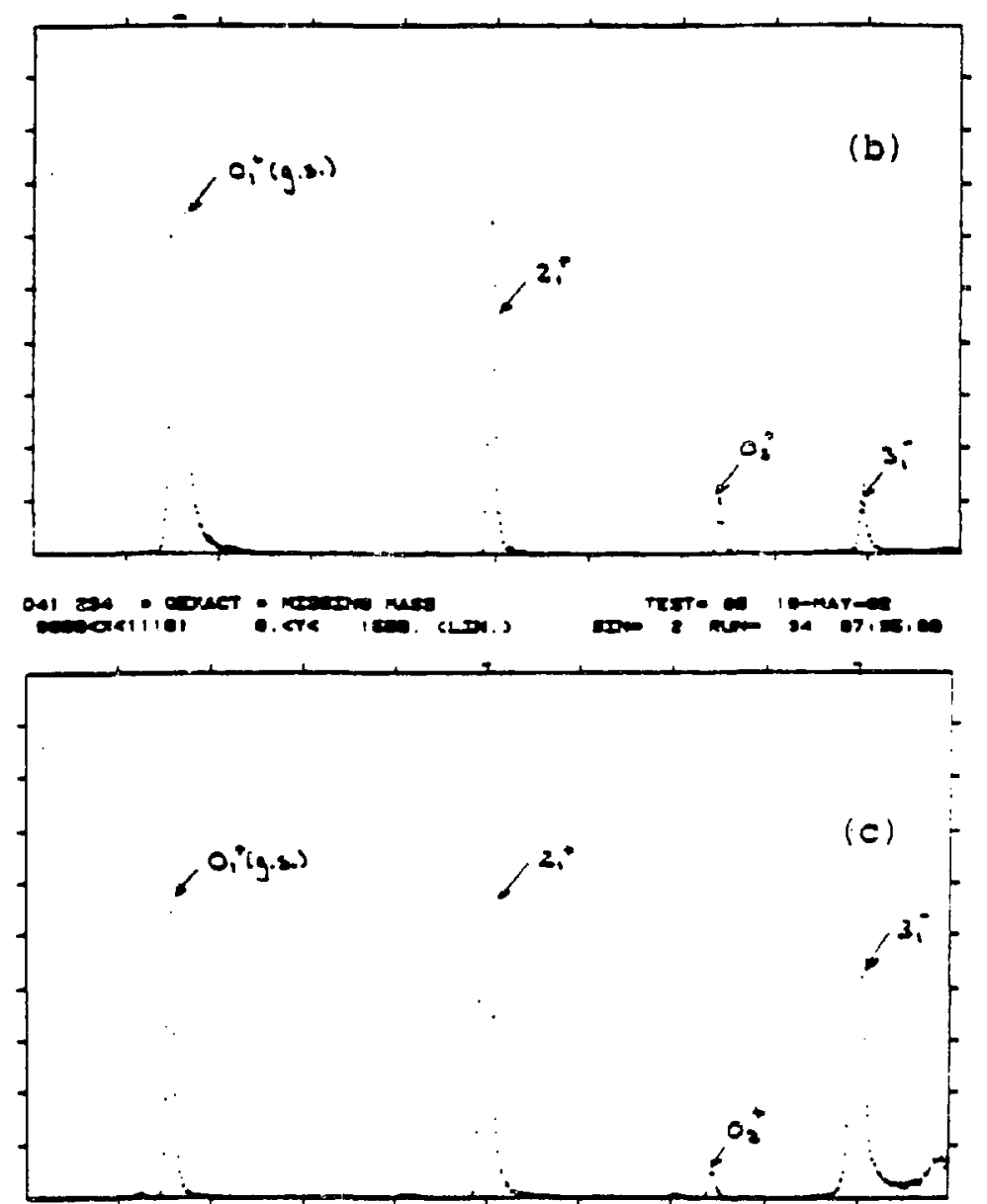

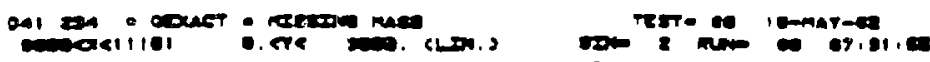

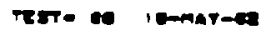
Figure $\mathrm{V}-1$. 
ground is negligible up to an excltation energy of some 7-8 Mev, but starts to rise smoothiy above this energy. The spectrum at $q=1.75 \mathrm{fm}^{-1}$ shows a marked decrease in strength for both elastic scattering and the $\theta_{2}^{+}$state while the $2_{i}^{+}$state is now dominant. The baskground is similar to that observed at $q=0.97 \mathrm{fm}^{-1}$.

Figure $V-2$ (a) show an excitation range from 7.0 to $18.0 \mathrm{MeV}$ at $q=0.34 \mathrm{fm}^{-1}$. It is apparent that the dominant inelastic states in this region are the unnatural parity $1^{+} ; 0.1$ states at $E_{x}=12.71 \mathrm{MeV}$ and $E_{x}=15.11 \mathrm{MeV}$ respectiveiy. The $\theta_{2}^{+}$state is aiso seen. The background shows no significant structure, and at this smail laboratory angle $\left(4^{\circ}\right)$ the uniform background is attributable to edge scatterIng of particies entering the spectrometer acceptance. It should be noted that these states observed here require no anguiar momentum transfer to the nucleus $(\Delta L=\theta)$, and it is therefore not surprising that they shouid dominate the low momentum transfer inelastic scattering.

The spectrum in Eigure $V-2(b)$, at $q=0.97 \mathrm{fm}^{-1}$, shows considerabiy more structure than that at in Figure v2 (a). Isoscalar states of natural parlty are now dominant, especiaily the $\theta_{2}^{+}, 3_{1}^{-}$and $1_{1}^{-}$states $\left(E_{X}=7.65,9.64\right.$ and $10.84 \mathrm{MeV}$ respectiveiy). The $4_{1}^{+}$state $\left(E_{x}=14.68 \mathrm{MeV}\right)$ is weakiy excited and the very broad ( -1 MeV) collective quadrupole state at $15.3 \mathrm{MeV}$ is clearly evident. The $1^{+}$states 
Medium Excitation Spectra
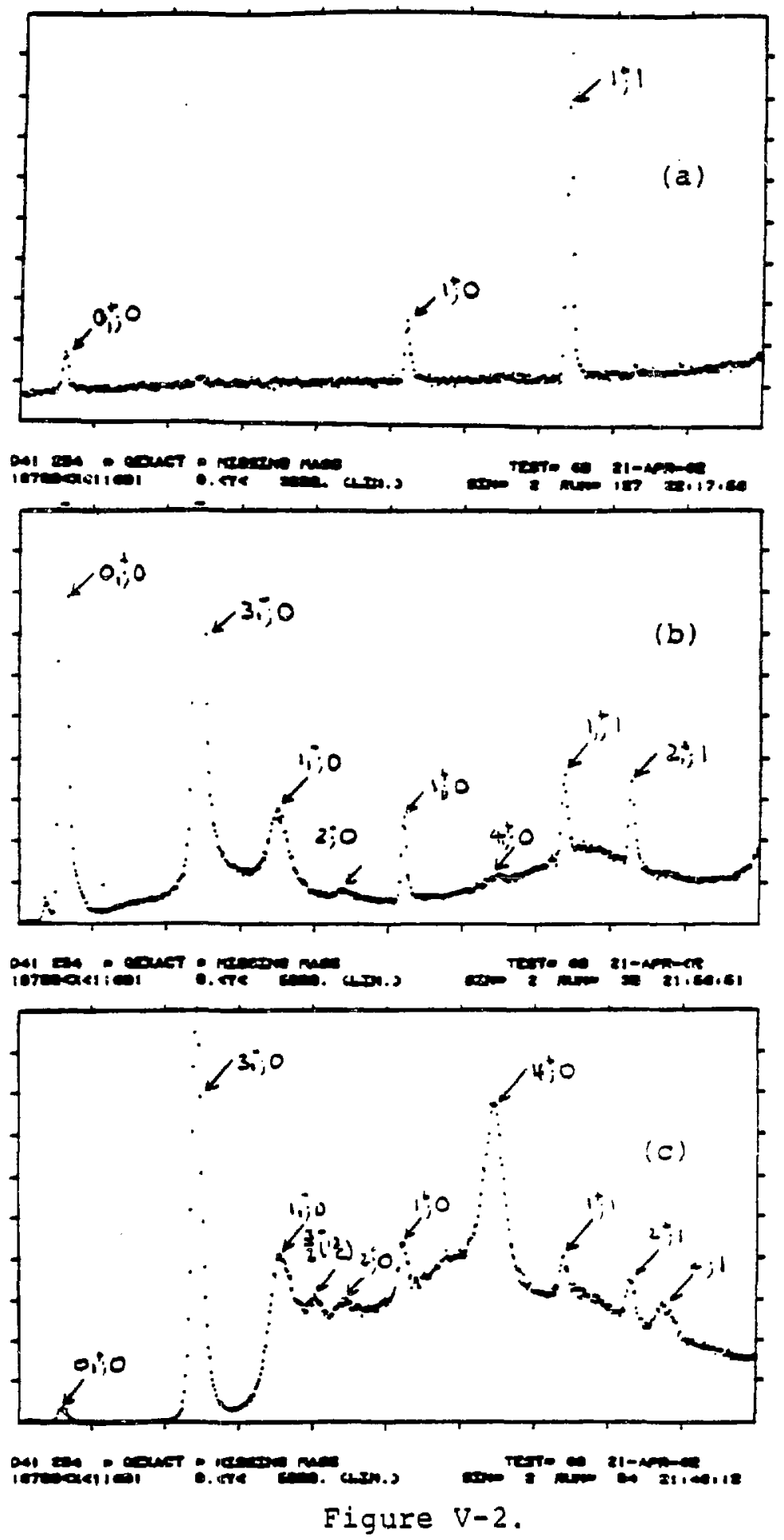
remain visibie aithough it is clear that their strength is diminishing. Also, the relative strength of the isoscalar $1^{+}$state is approximately the same as the isovector $1^{+}$ state, whereas at smali $q$ the 1 sovector state is much strorger (see figure $\mathrm{V}-2(\mathrm{a})$ ). The isovector $2_{I}^{+}$state at $\Sigma_{x}$ $=16.11 \mathrm{MeV}$ is now visible, and a state at $11.83 \mathrm{MeV}$ is also seen.

Note that the background at $7 \mathrm{MeV}$ of excitaticn is smail, and that the "smali peak to the left of the $\theta_{2}^{+}$state is evidence of the excitation of a $\frac{3^{+}}{2}$ state in ${ }^{13} \mathrm{C}$ at $E_{x}=$ 7.68 MeV due to the presence of this isotope in the naturai graphite target.

Figure $V-2$ (c) shows a spectrum for the same excitation region at $q=1.75 \mathrm{fm}^{-1}$. The MBD cut discussed in the preceding chapters can eastiy be seen at an excltation energy of about $10 \mathrm{MeV}$, interfering directiy with the $1_{i}^{-}$state at $E_{X}=10.84 \mathrm{MeV}$. The spectrum is again dominated by natural parity isoscalar states, most notably the $3_{1}^{-}$and $4_{1}^{+}$ states, while the $1^{\text {t }}$ states are depressed relative to previous spectra ar smailer $q$. The lowest 2 - isovector state at $\varepsilon_{x}=16.58 \mathrm{MeV}$ is now visible. The $7.68 \mathrm{MeV}$ state in ${ }^{13} \mathrm{C}$ has kinematically shifted to lower excitation energy, while an apparent doublet has developed in the 11-12 MeV excitation energy region. We intirpret this doubiet as consisting of the isoscaiar $2^{-}$state in ${ }^{12} \mathrm{C}$ at $11.83 \mathrm{MeV}$ and a kinemat- 
icaliy shifted state of ${ }^{13} \mathrm{C}$ at $11.46 \mathrm{MeV}$ which 1 tself $1 \mathrm{~s}$ an uncesolved doublet of spins $\frac{5}{2}^{+}$and $\frac{7}{2}^{+}$. This identification is made upon observation of the kinematic shift of the 'state' as a function of $q$, and the fact that considerabie strength in the region has been observed in 550 MeV inelastic proton scattering from ${ }^{13} \mathrm{C}$ as shown in Figure $\mathrm{V}-4^{43)}$. We also note that the glant quadrupole state at $15.3 \mathrm{MeV}$, aithough stili seen, is much weaker. It appears that some broad strength is present at $-13.5 \mathrm{MeV}$ of excitation. There is an isoscalar $2^{-}$state of ${ }^{12} \mathrm{C}$ at $13.35 \mathrm{MeV}$ with a width of some $400 \mathrm{keV}$. This is much narrower than the broad structure observed in the spectrum, and cannot account for this strength. The apparent shift in coilective strength Erom $15.3 \mathrm{MeV}$ to a iower excitation energy is observed at ali beam energies, and appears to be a reai effect.

Nuciear data tabies ${ }^{44}$ ) show there to be a very broad state in ${ }^{13} \mathrm{C}$ at about $13.4 \mathrm{MeV}$, but examination of Figure $V-4$, which is representative of a roughiy equivalent momentum transfer to that of Elgure $v-2(b)$, shows insufflcient strength in the region to warrant identification of the broad state seen in this work with an ineiastic excitation of a broad state in ${ }^{13} \mathrm{C}$. A further point against such an identiflcation is the small fraction of ${ }^{13} \mathrm{C}$ present in the target, as weil as the relative strength of the $13.4 \mathrm{MeV}$ region compared to the other states of ${ }^{13} \mathrm{C}$ visibie in our spectra. 
Figure $V-3$ siows spectra for the excitation energy range from 17.0 to $21.0 \mathrm{Mev}$. The 18.0 to $21.0 \mathrm{MeV}$ region is seen to contain several broad states. There is a state at $18.3 \mathrm{MeV}$ of width $380 \pm 30 \mathrm{keV}$ which is seen in ali spectra from smailest to largest values of $g$. The various states in the 19 MeV regton whlch have been described prevlousiy are cleariy seen. A state at $E_{x}=20.6 \mathrm{MeV}$ of widh $280 \pm 20$ keV is seen for values of $q$ greater than $-0.85 \mathrm{fm}^{-1}$.

B) Differential Cross-sections and Analyzing Powers

1) Isoscaiar states of natural parity

We discuss here the data for states with $\pi=(-1)^{\mathrm{J}}$ which are predominantly excited by mechanisms which do not involve spin or isospin trangfer to the nucieus $(\Delta S=0 ; \Delta T$ $=0)$. The states of interest in this ciass are $\theta_{1}^{+}(0.00)$, $2_{1}^{+}(4.44), 0_{2}^{+}(7.65), 3_{1}^{-}(9.64), 1_{1}^{-}(10.84)$ and $4_{1}^{+}(34.08)$ where the number in parentheses indicates the excitation energy of the residual nucleus.

(a) Elastic scattering $\left(\theta_{1}^{+} ; \theta-E_{x}=0.00 \mathrm{MeV}\right)$

The data are presented in Figures $V-5$ to $V-7$. The differential cross sections at all beam energles show the characteristic opaque disc dfffraction pattern assoclated with elastic scattering. Also, the magnitude of the cross section generaliy increases as an increasing function of incident beam energy. The value of momentum transfer $q$ at 
High Excitation Spectra

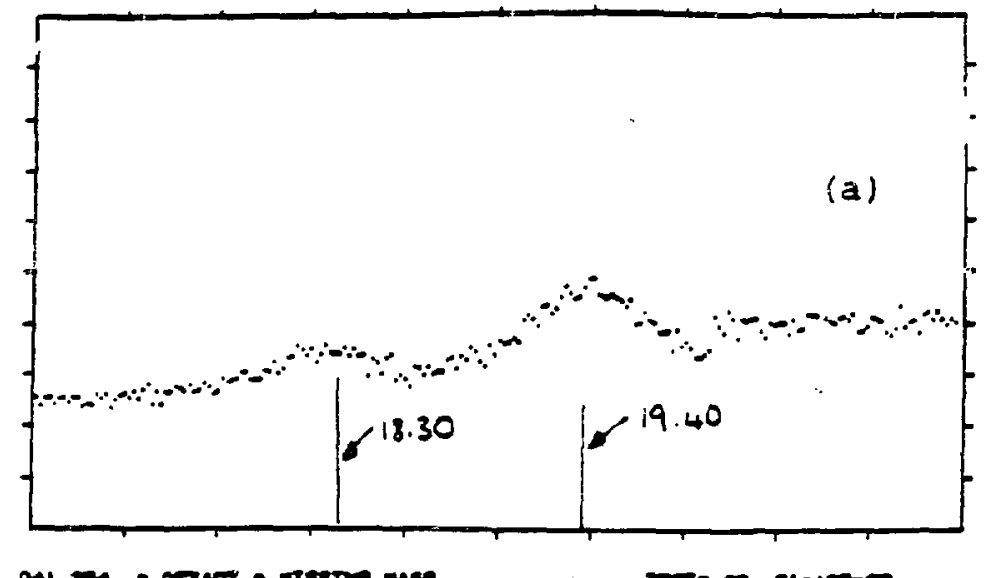

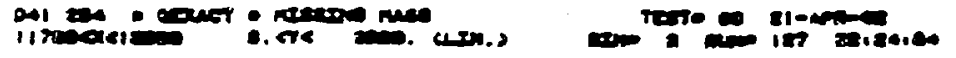

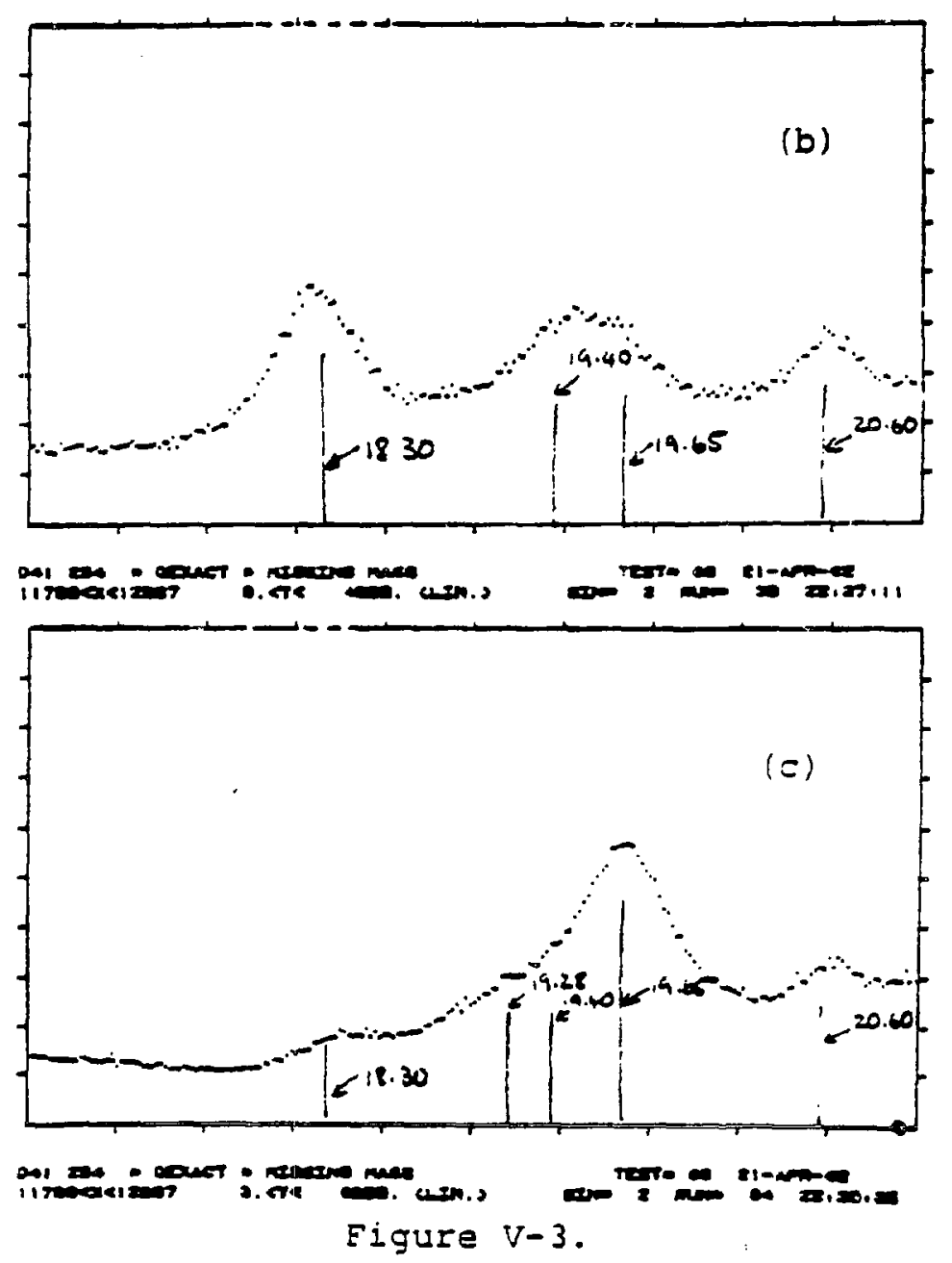




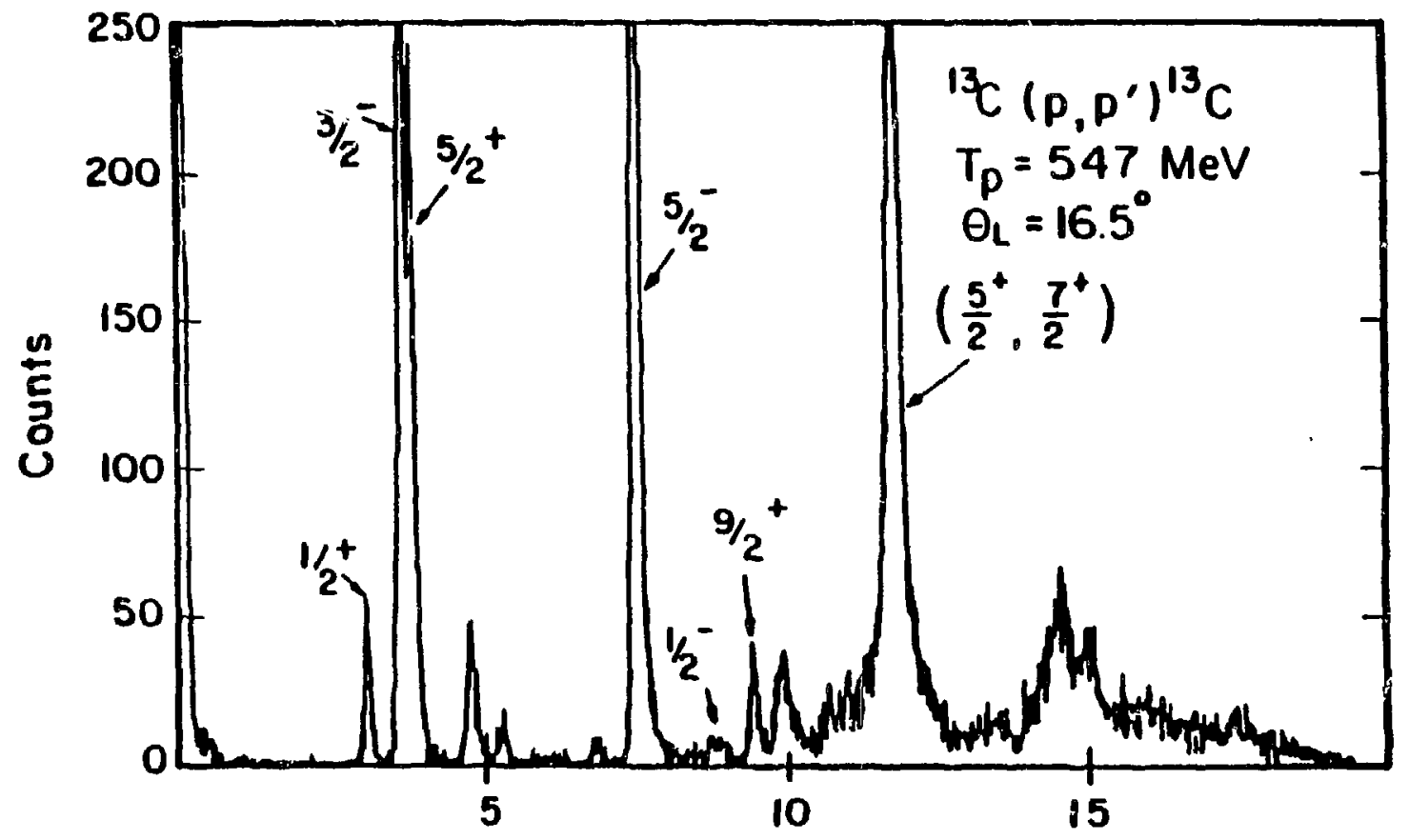

Excitation Energy (MeV)

Figure $v-9$. 
which the first minimum in the cross section is iocated decreases silghtiy as the incident proton energy increases, and the shape of the mintmum becomes somewhat narrower.

Simfiar phenomena are seen in the analyzing power data, in which the value of $q$ for which the sharp rise corresponding to the cross section minimum has $A_{y}=0$ decreases as the incident proton energy increases. In general, the positive region of $A_{y}$ for $q \leq 1.2 \mathrm{fm}^{-1}$ decreases in magnitude as the beam energy increases, while also showing iess pronounced curvature. The depth of the minimum decreases as $\varepsilon_{p}$ increases, consistent with the previousiy known Eact that $A_{y}$ is strictiy positive in the measured range of $q$ for $E_{p}=800 \mathrm{MeV}^{45}$. Of interest is the observation that, at $398 \mathrm{MeV}$, the vaiue of $\mathrm{A}_{y}$ osciiiates from -1 to +1 while passing through the position of the minimum in the cross section. Such extreme osciilation may have appilication as an energy calibration point.

On first appearance it seems as though the 'width' of the minumum in $A_{y}$ is increasing as the beam energy increases. We believe this to be iliusory, resuiting from the decrease in positive vaiues for iow $q$, and the shift to lower $q$ of the crossover point from negative to positive vaiues of $A_{y}$. If ali trree minima are aligned, the fali-off to the most negative vaiue of $A_{y}$ occurs with the same siope for aij three beam energies. 
Elastic Djfferential crosa sections
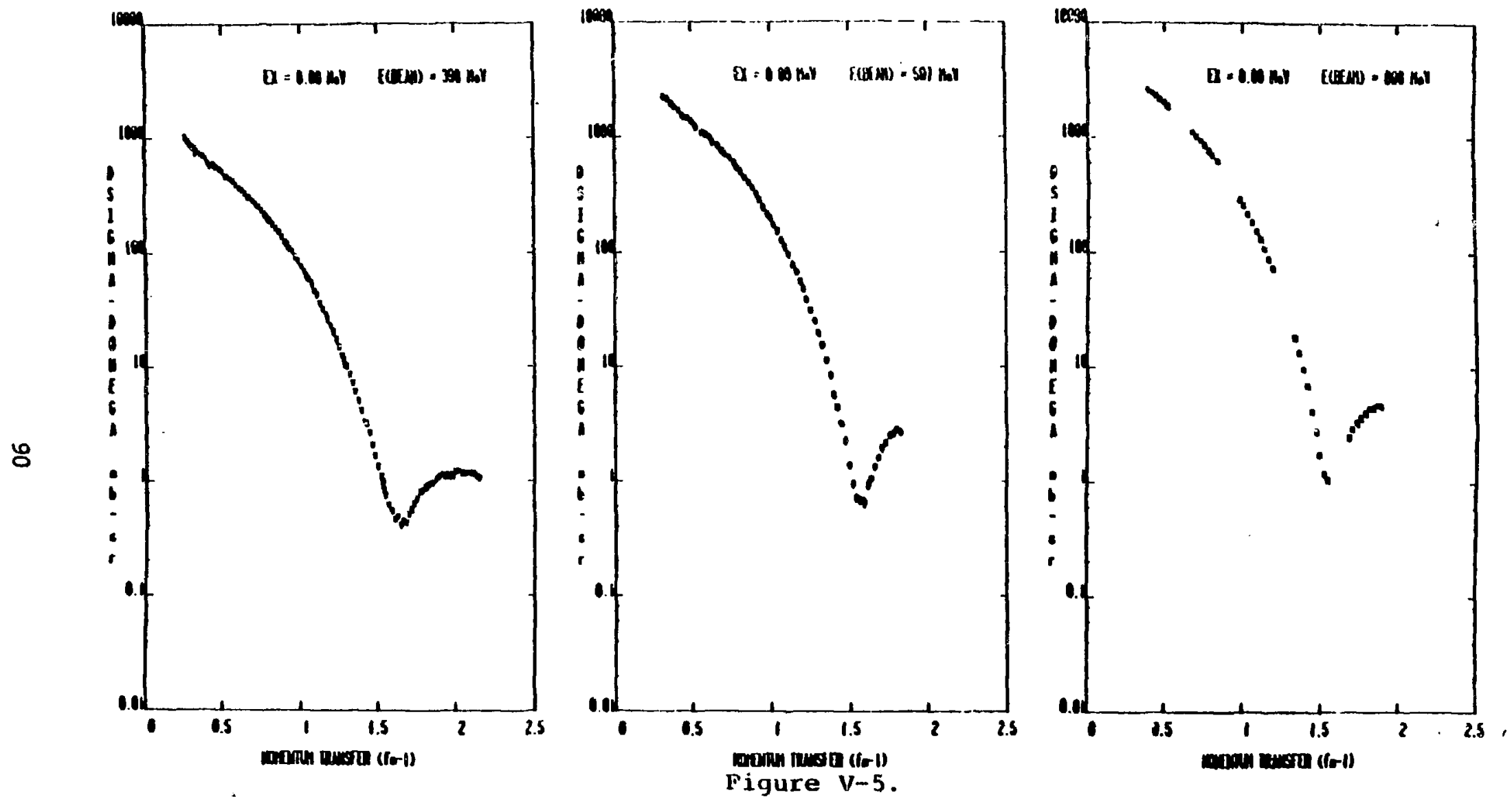
Elastic Analyzing Powers
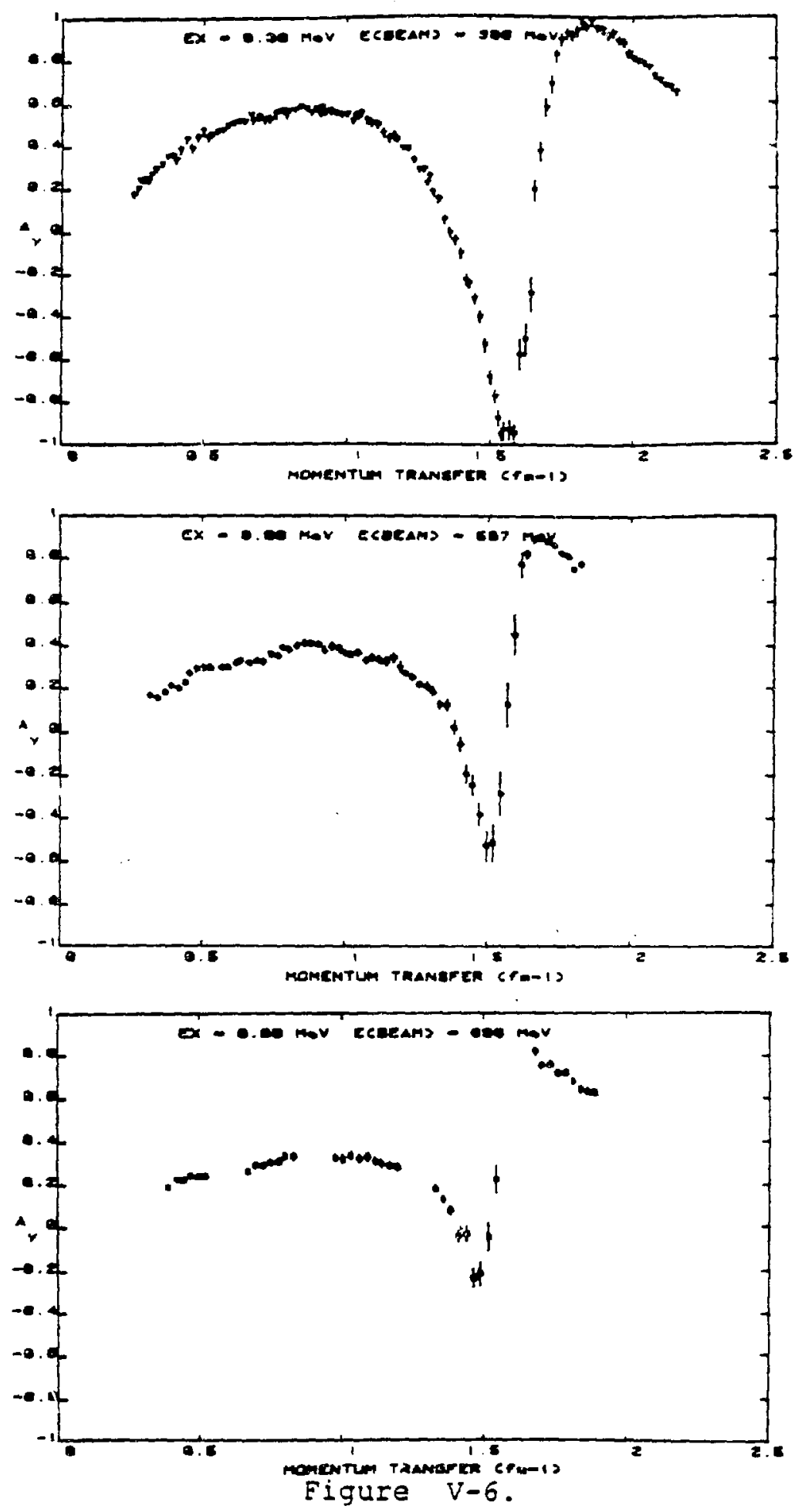


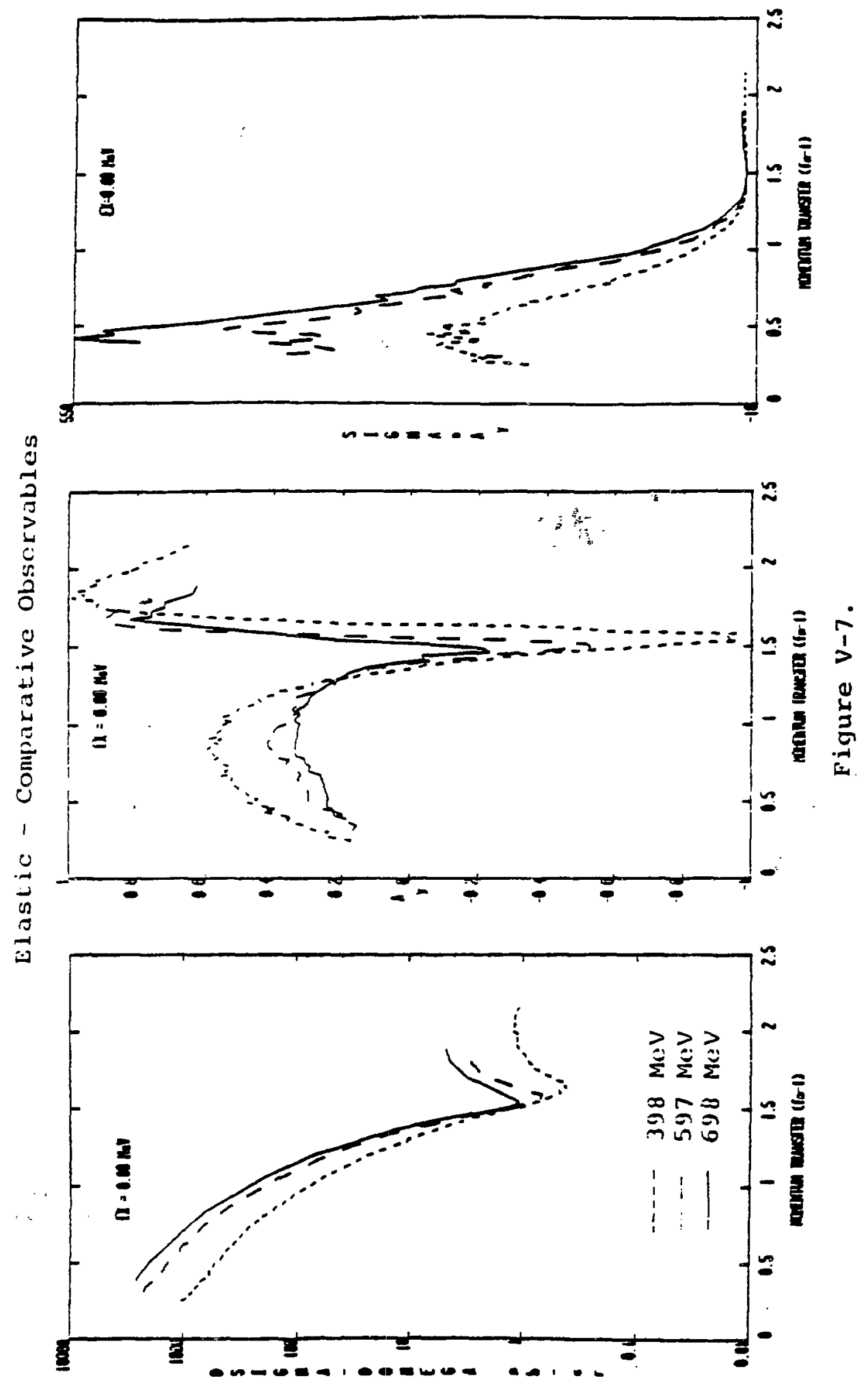


Simpie opticai model theory in which the nucieus is treated as a sharp opaque diss of radius $R$ predicts, to flrst order, a minimum in the observed differentiai crass section for a vaiue of $q R$ such that

$$
\frac{J_{1}(q R)}{q R}=0 \quad v-1
$$

where $J_{l}(q R)$ is a Bessel function of the first kind. The value $q R=3.8$ gives this condition. Noting that $q$ is about $1.55 \mathrm{fm}^{-1}$ on average for the location of the first minimum in the eiastic differential cross section, we find a value $r_{0}=1.08 \mathrm{fm}^{-1}$, where $R=r_{0} A^{(1 / 3)}$. This rough vaiue is consistent with parameters obtained in fuil optical model search caicuiations described in the next chapter. We do not beileve it to be appropriate to attempt to expiain the smaif shift in the minimum as a function of $q$ in terms of this simpie approximation, because diffuseness effects and the weil-known energy dependence of the opticai model parameters have not been taken into account.

It is instructive to consider the behaviour of the product of the differential cross section and the anaiyzing power as a function of the incident beam energy, as shown in Figure $v-7(c)$. We note that for $q \geq 1.3 \mathrm{fm}^{-1}$ this product is constant for all beam energies, and that each curve peaks at $q \equiv 0.5 \mathrm{fm}^{-1}$. The peak ratios, normaiized to that at $\varepsilon_{p}$ $=398 \mathrm{MeV}$, are $2,1: 1.6: 1.0$. 
We should note that our data for the differential cross sections at $E_{p}=398$ and $597 \mathrm{MeV}$ respectively are in good agreement with those obtained by others at 402 and 600 Mev. These other experiments were performed with unpoiarized beam, and consequently anaiyzing powers were not available for comparison.

(b) The $2_{1}^{+} ; 0$ state at $E_{x}=4.44 \mathrm{MeV}$

The differential cross sections data are presented in Elgure $V-8$, and the analyzing power data are presented in Figure $V-9$. In addition, Figure $V-10$ shows the data for ail beam frergies plotted sumuitaneously, as weil as the product * A for ali beam energies.

We notice that the differentiai cross sections for incident beam energies of 597 and $698 \mathrm{MeV}$ are iarger than that at $398 \mathrm{MeV}$ by Eactors of 2.8 and 3.5 in the region of the maximum. The location of the maximum aiso exhibits a shift to smalier $q$ for increasing beam energy in the same fashion as the minimum in the eiastic data. of interest is the shape of the measured differentiai cross section for $q ?$ $1.5 \mathrm{fm}^{-1}$.. While the shape below this value is roughiy constant for all beam energifs, the data at 398 MeV fali off iess rapidiy in this region of momentum transfer than those at 597 or $698 \mathrm{MeV}$ i in fact, the valies are seen to coincide at $q-1.75 \mathrm{sm}^{-1}$.

The anaiyzing powers are alway positive in the meas- 
$\mathbf{E}_{x}=4.44 \mathrm{MeV} 2{ }_{l}^{\prime} ; 0-$ Differential cross Sections
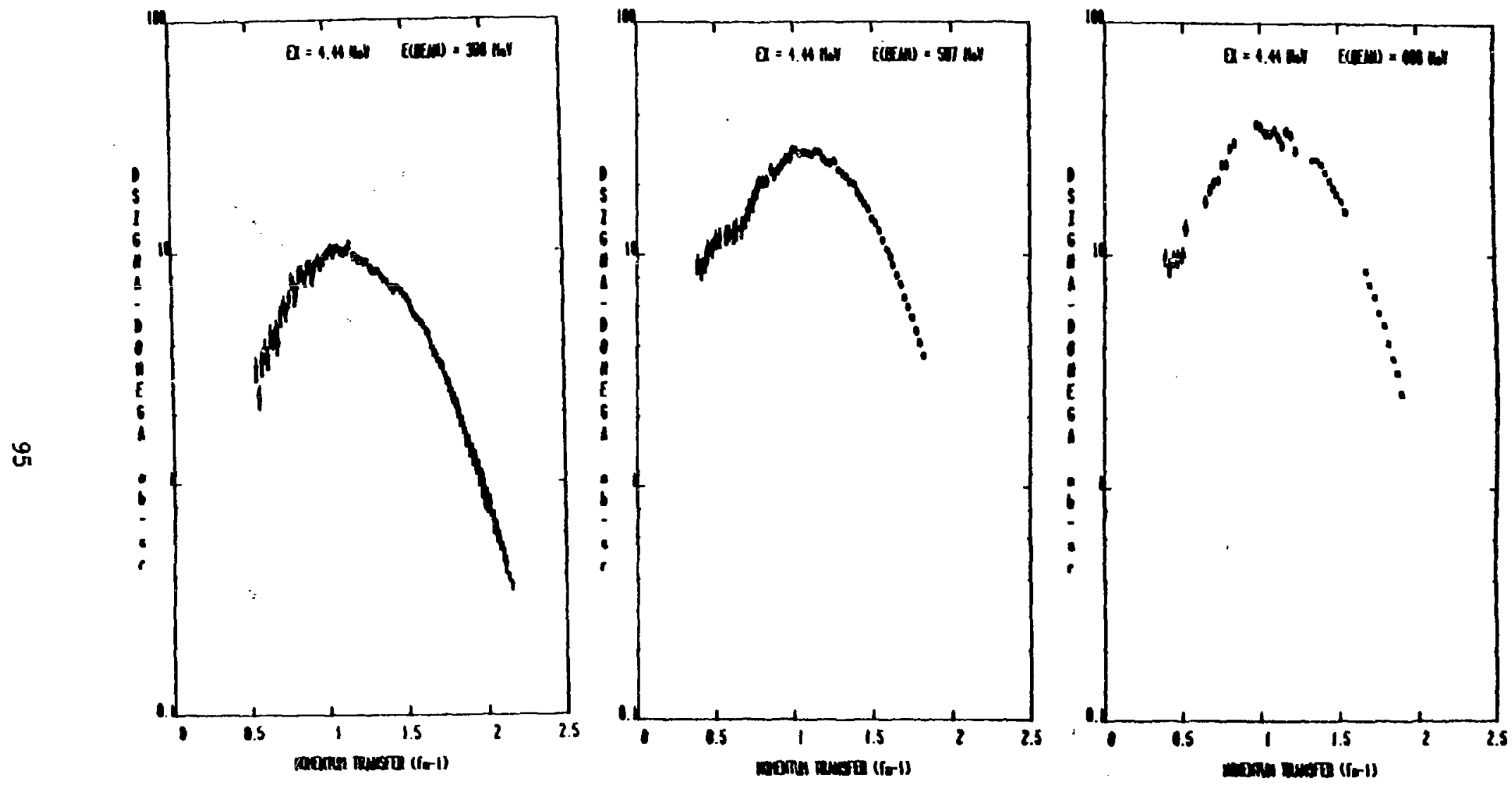

Fiqure $\mathrm{V}-8$. 

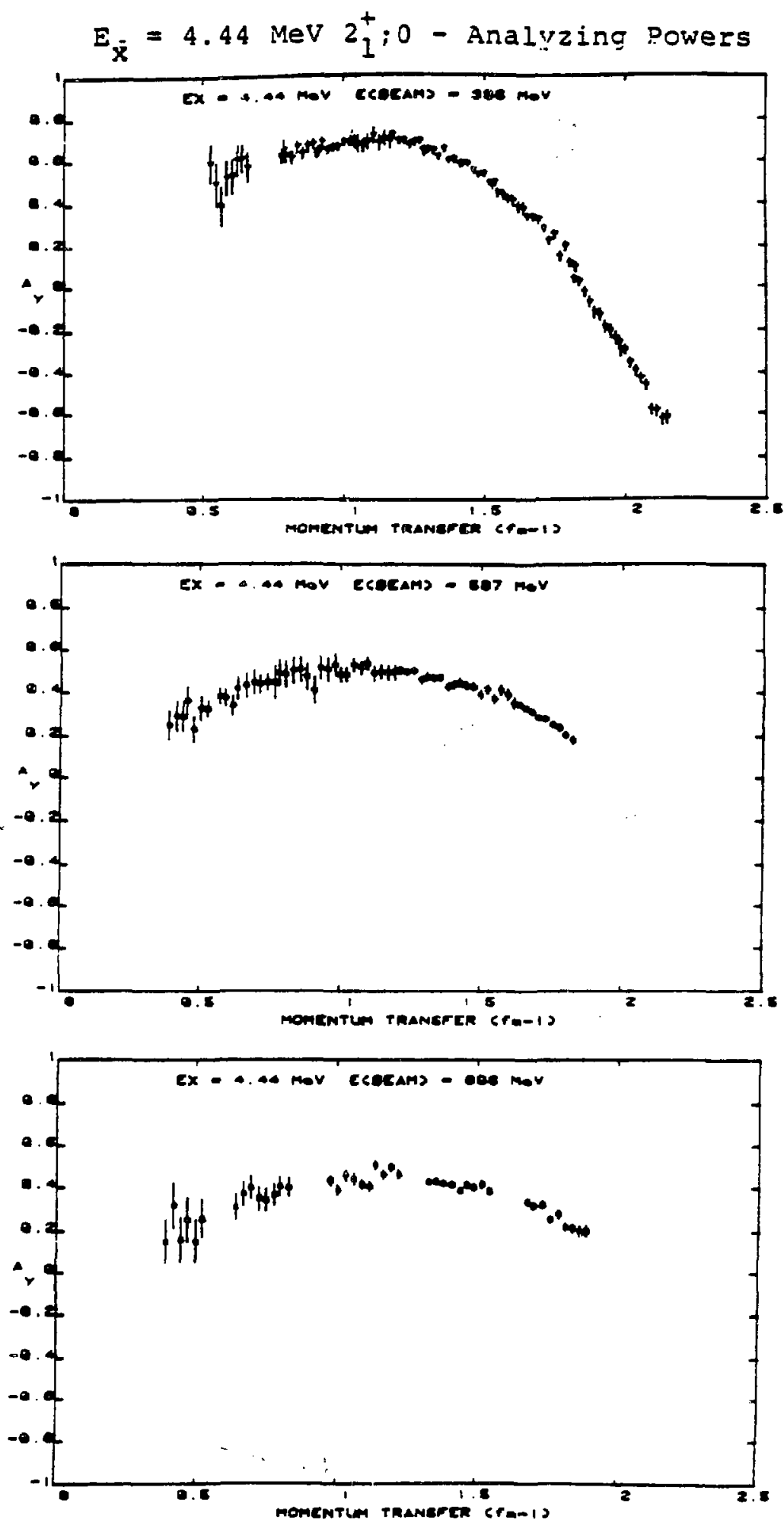

Figure V-9. 


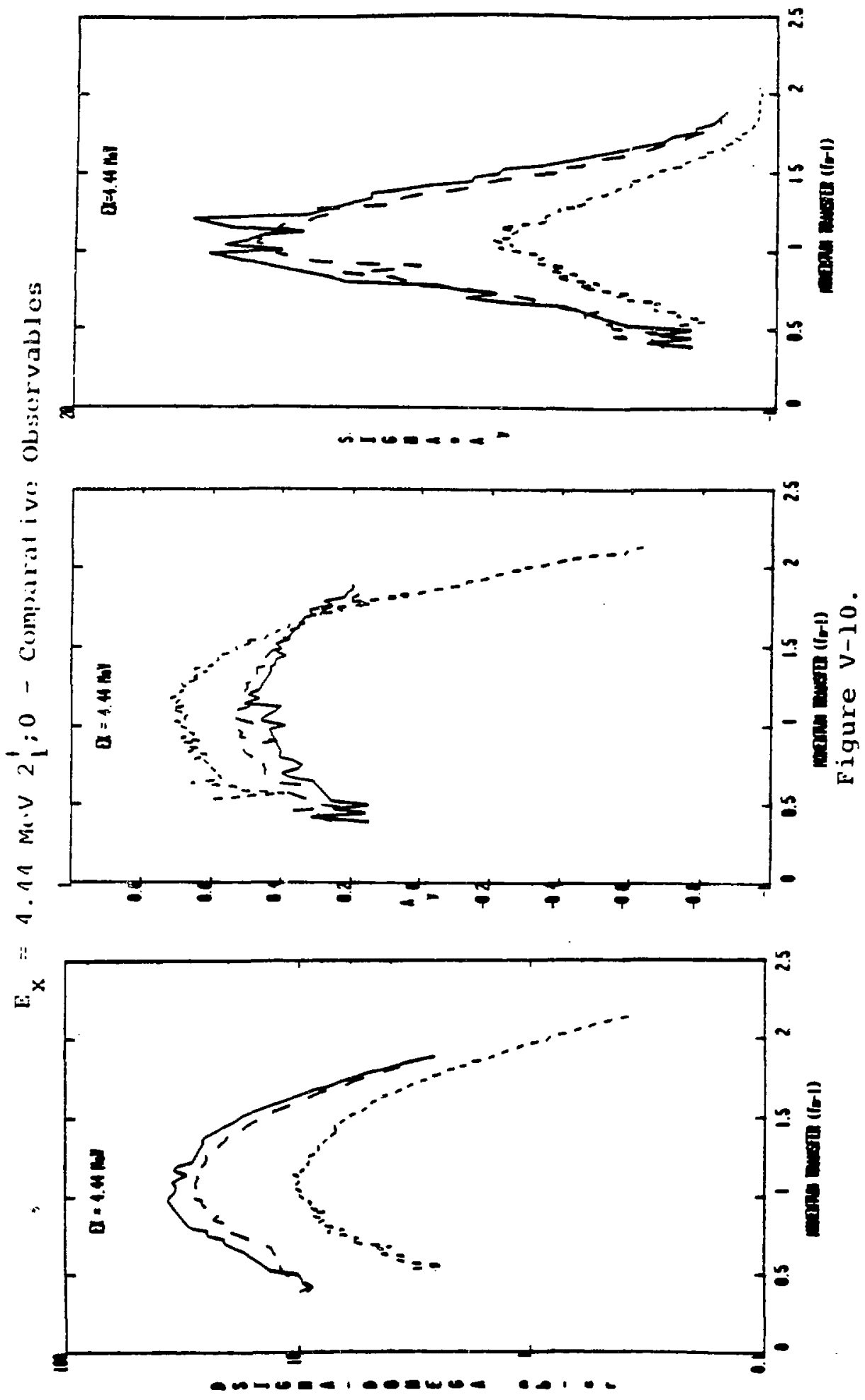


ured region for the two higher beam energles, while that at $398 \mathrm{MeV}$ becomes strongly negative in the $q=2.0 \mathrm{fm}^{-1}$ region. We note that the magnitude of the analyzing power decreases for increasing beam energy in the region $0.5 \mathrm{fm}^{-1} \leq$ $q \leq 1.5 \mathrm{fm}^{-1}$, with the difference between the data at 597 and $698 \mathrm{MeV}$ being much Iess pronounced than that between these data and those at 398 MeV.

Study of the data for $\sigma$ * $A_{y}$ shows strong peaking in the $q=1.05 \mathrm{fm}^{-1}$ region for all beam energies. The large $q$ values for this quantity at $E_{p}=398 \mathrm{MeV}$ are sightiy negative, reflecting the change of sign sf the analyzing power in this region. In keeping with the behaviour of the two observables, the product exhibits almost similar values for $E_{p}=597$ and $698 \mathrm{MeV}$, which are much enhanced over those for $E_{p}=398 \mathrm{MeV}$. Examination of these data yield the following ratios at the peak normaiized to that for $E_{p}=398 \mathrm{MeV}$; $2.13: 1.95: 1$.

(c) The $\theta_{2}^{+} ; 0$ state at $\varepsilon_{x}=7.65 \mathrm{MeV}$

The differential cross section data are presented in Figure $V-11$, the analyzing power data in Figure $V-12$ and Elgure $V-13$ shows these data plotted together as well as the product $\sigma * A_{y}$ for all beam energies.

The differential cross section shows a strong peak Eor ali beam energies at $\xi=1.0 \mathrm{fm}^{-1}$. The maximum values for $\sigma$ rise as the beam energy increases, yieiding ratios at 

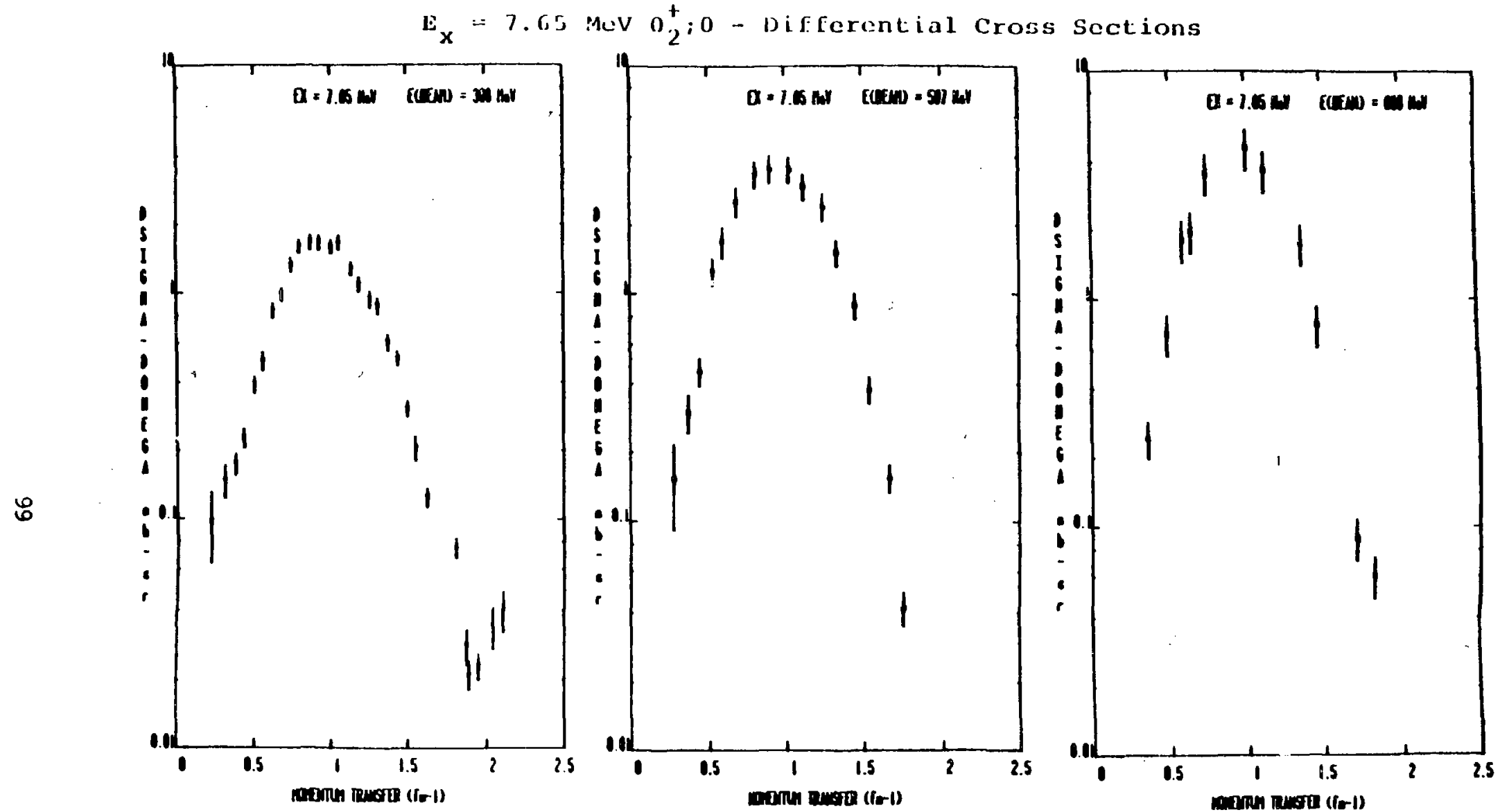

Figure V-11. 

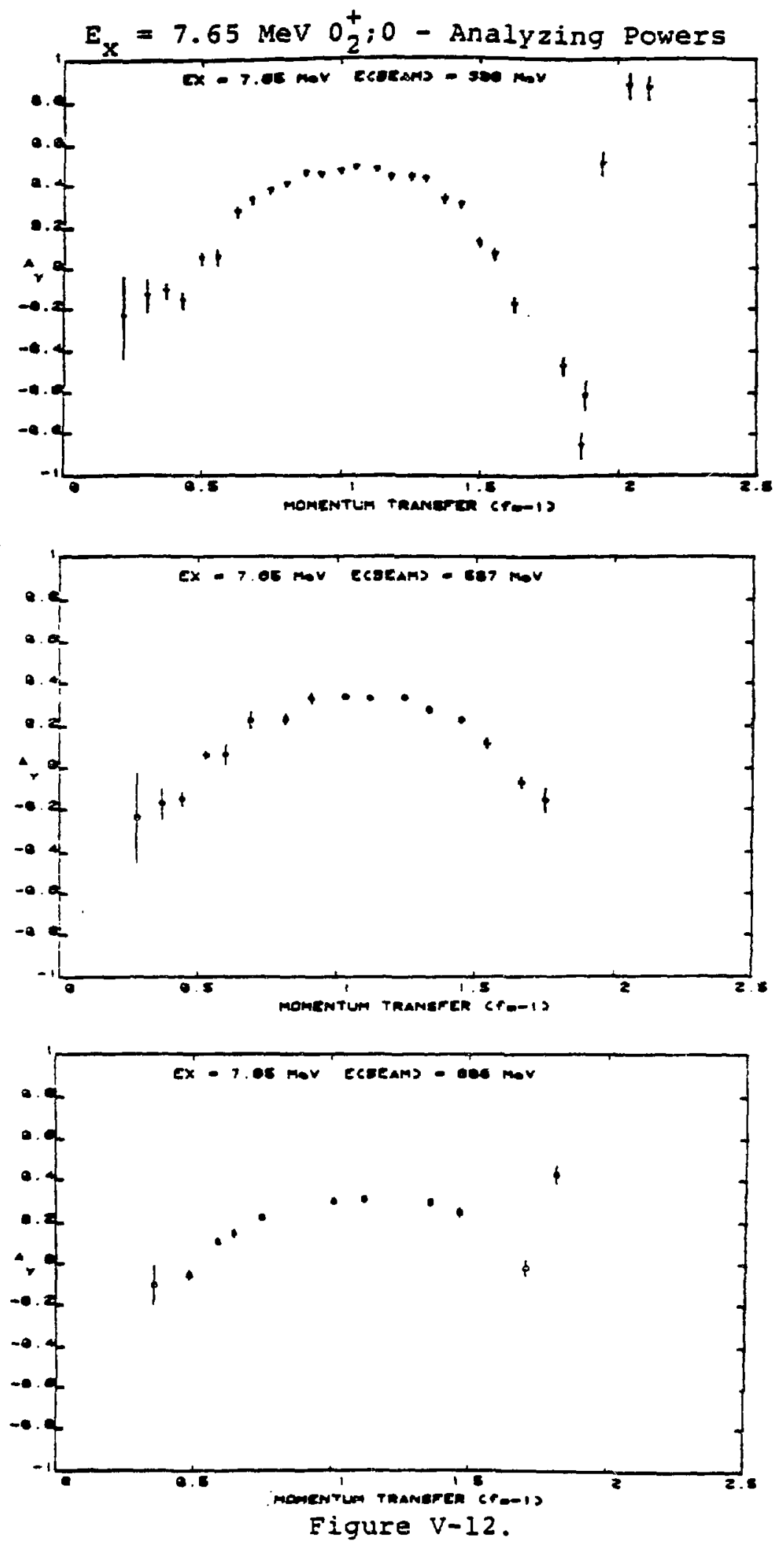
$\mathrm{s}_{\mathrm{x}}=7.65 \mathrm{MeV} 0_{2}^{t}: 0$ - comparative Observables
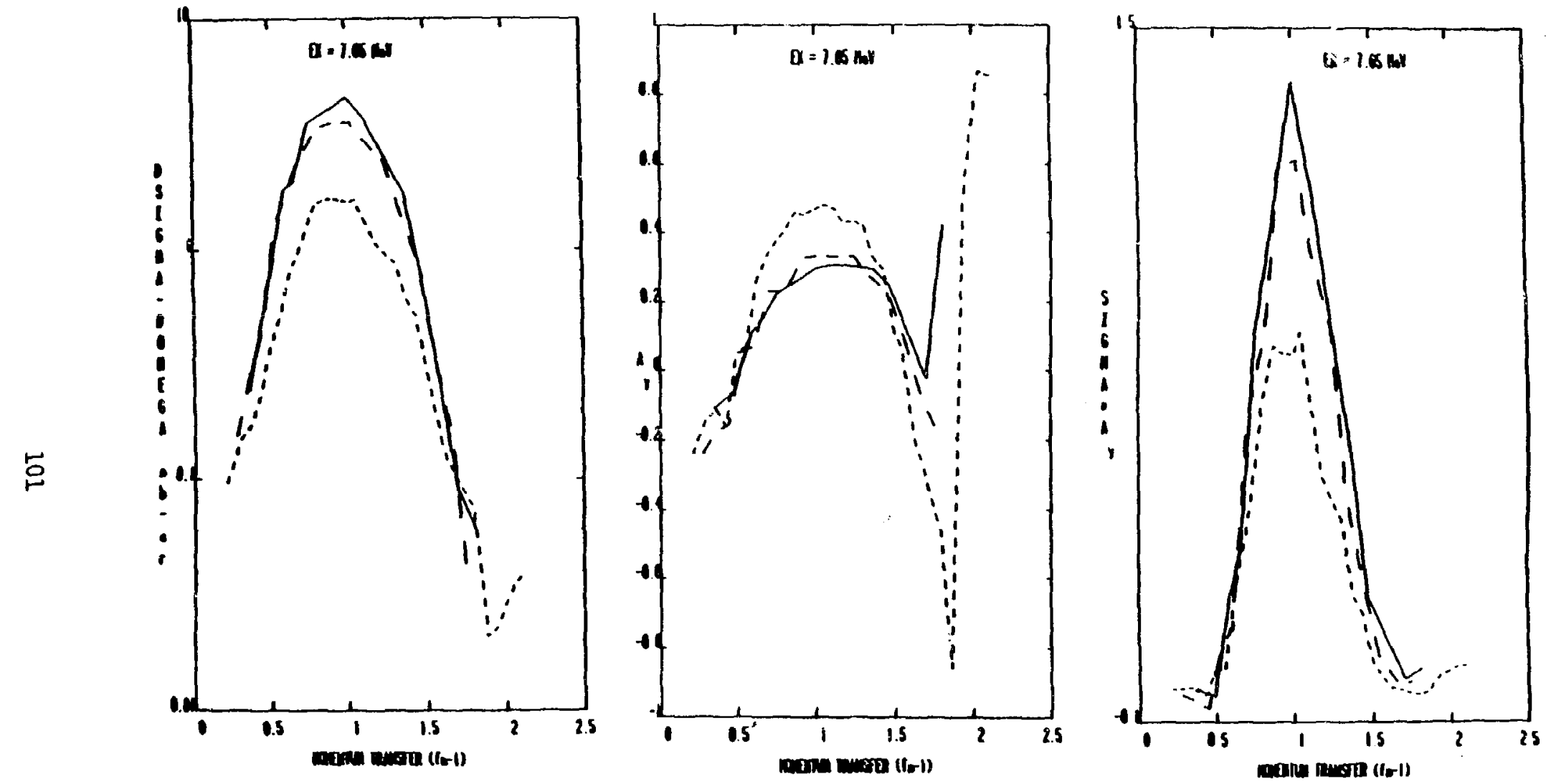

Figure $v-1 j$. 
the peak normalized to that for $\varepsilon_{p}=398 \mathrm{MeV}$ of $2.8: 2.1: 1.0$. At $E_{p}=398 \mathrm{MeV}$ we note a minimum in the differential cross section at $q=1.9 \mathrm{fm}^{-1}$, after whlch the value of the cross section is seen to rise once more. The data at $E_{p}=597$ and $698 \mathrm{MeV}$ do not extend sufficientiy far in $q$ to show this effect.

The analyzing powers are negative for aij bean energies from the smallest value of $q$ for which data were taken out to $9-0.55 \mathrm{fm}^{-1}$. Thereafter, $\mathrm{A}_{\mathrm{y}}$ rises smoothiy to a positive maximum vaiue at $9-1.15 \mathrm{fm}^{-1}$ for all values of $\mathrm{sp}_{\mathrm{p}}$. The data at $E_{p}=398 \mathrm{MeV}$ change sign at $q=1.55 \mathrm{fm}^{-1}$, dropping to -0.9 at $q=1.9 \mathrm{fm}^{-1}$. For $1.9 \mathrm{fm}^{-1} \leq q \leq 2.05 \mathrm{fm}^{-1}$ there is a sharp rlse in $A_{y}$ to +0.94 reminiscent of the behaviour of the elastlc analyzing power in the region of the minimum in the differential cross section. We note that this sharp rise occurs at the same vaiue of $q$ as the observed minimum in the differentiai cross section, in keeping with the elastic scattering data. The data at $E_{p}=597 \mathrm{MeV}$ aiso change sign at $q=1.55 \mathrm{fm}^{-1}$, although the region in which the sharp ise night be seen was not examined. For $\varepsilon_{p}$ = $698 \mathrm{Mer}$ a small negative value is seen for $9-1.7 \mathrm{fm}^{-1}$, and the last data point shows a sharp change in sign to +0.4 . This would indicat a shift to a sightiy smaller value of $q$ for this sharp rise in the analyzing power at this beam energy. We note that the basic structure of the for the elastic scattering data. The depth of the minimum decreases as 
beam energy increases, while the crossoover point from negative to positive values of $A_{y}$ in the sharp rise apparentiy shifts to Iower $q$ for increasing beam energies. Further comparison with the elastic data indicate that the minimum in $\frac{d \sigma}{d \Omega}$, together with the 'osciliation' in $A_{Y}$ occur at a larger value of $q$ for this state than that for which the effect is seen in the elastic data.

As has been seen for the other state examined this far, the maximum value of $A_{y}$ in the smoothiy positive region is seen to decrease as a function of increasing beam energy, the maximum occurring at $q-1.05 \mathrm{fm}^{-1}$. Again, the difference between values for $E_{p}=398 \mathrm{MeV}$ and $597 \mathrm{MeV}$ is much iarger than that between values for $\varepsilon_{p}=597$ and $698 \mathrm{MeV}$.

The data for I *A show a behaviour very similar to that for the $2_{1}^{+} ; \theta$ state. A sharp peak is seen at $q=1.0$ $\mathrm{fm}^{-1}$, with the maximum value ratios being $1.8: 1.6: 1.0$. Negative vaiues are observed for $q \leq 0.5 \mathrm{fm}^{-1}$ and $q \geq 1.5 \mathrm{fm}^{-1}$, in keeping with the behavlour of the anaiyzing power in these regions of momentum transfer.

(d) The 3; ; 0 state at $E_{x}=9.64 \mathrm{MeV}$

Differential cross section data are presented in Figure V-14, and analyzing power data in Figure V-15. Figure $V-16$ shows the data at all beam energles plotted together, as well as the product $\sigma * A_{Y}$. 
$E_{x}=9.64 \mathrm{MeV} 3{ }_{1}^{-} ; 0$ - Differential Cross Sections
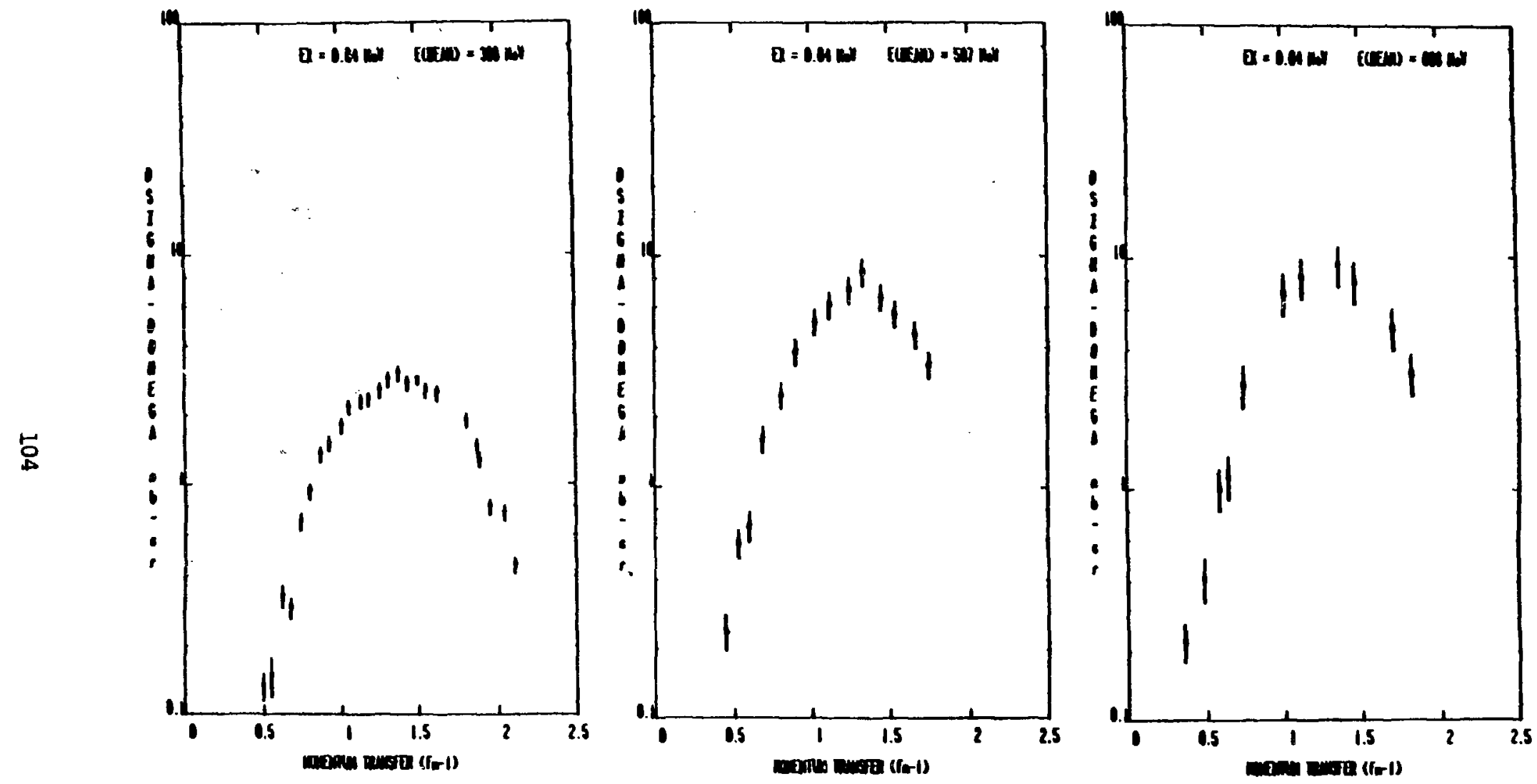

Figure V-14. 

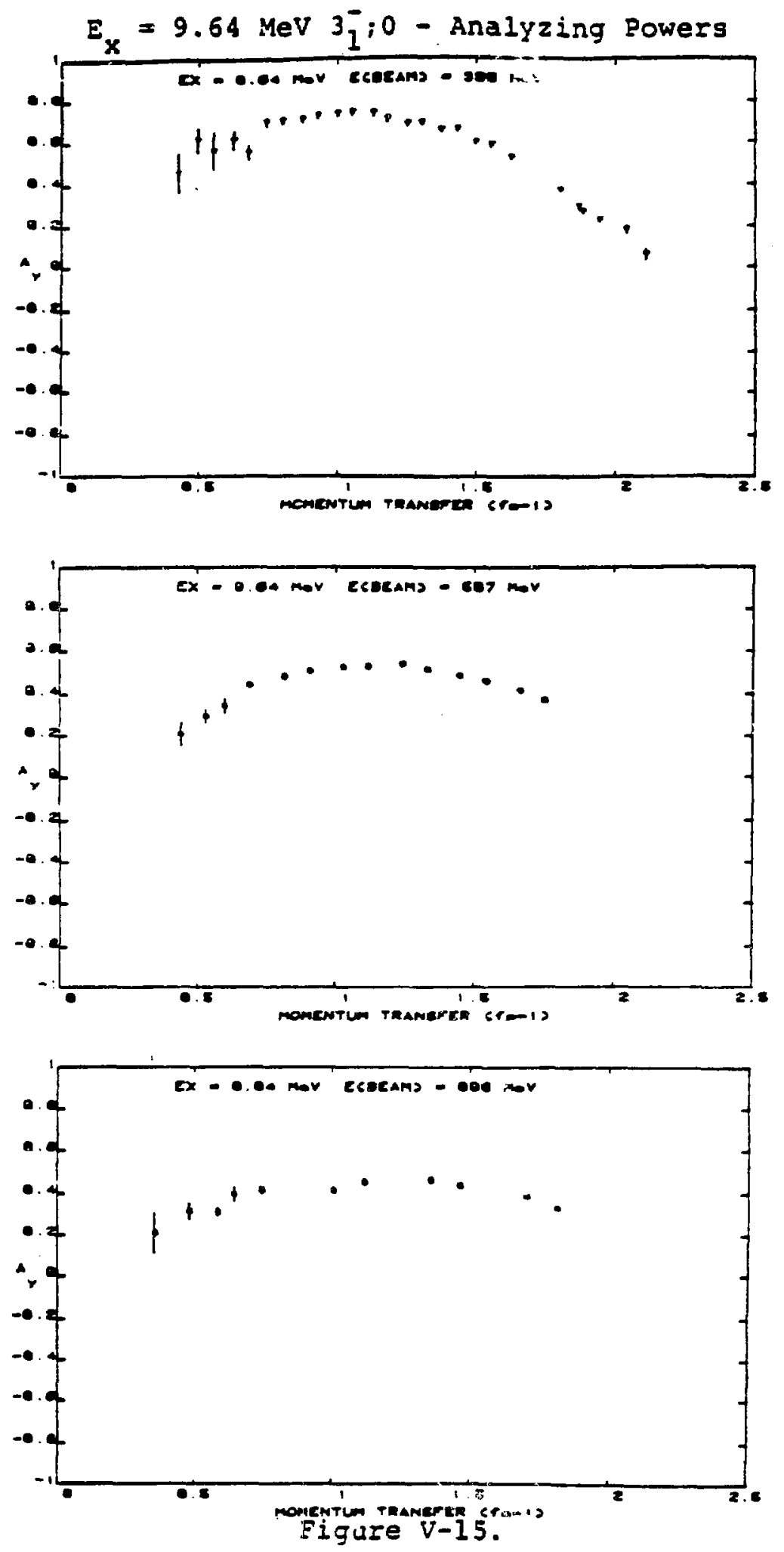
$E_{x}=9.64 \mathrm{MeV} 3 \overrightarrow{1} ; 0$ - Comparative Observables
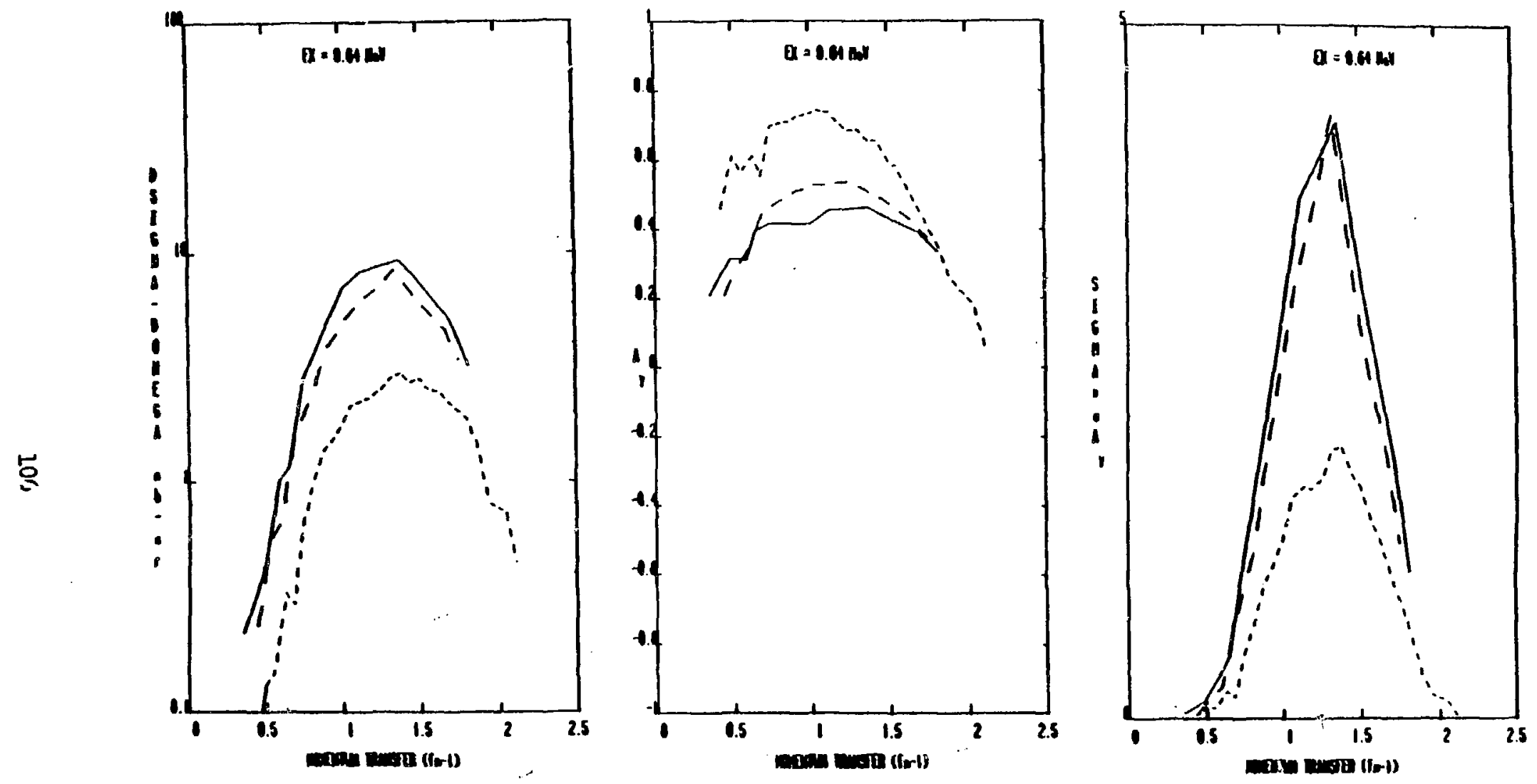

Fiqure V-16. 
The data for $\sigma$ show a smooth gise from $-0.1 \mathrm{mb} / \mathrm{sr}$ at $q=0.5 \mathrm{fm}^{-1}$ to a maximum value between 3 and $10 \mathrm{mb} / \mathrm{st}$ at $q-1.2 \mathrm{fm}^{-1}$ to $9-1.3 \mathrm{fm}^{-1}$. We see that the vaiue of 'q for which the differential cross section attains its greatest magnitude shows sight variation from one beam: energy to another. The maximum value of for the three beam energles are in the ratio 3.1:2.8:1.0. The shape of the differential cross section is consistent from beam energy to beam energy in the region of measurement.

The anaiyzing power data show little structure. In the measured region these data are aiways positive, rising smoothiy at low values of momentum transfer to maximum vaiues at $q-1.1-1.2 \mathrm{fm}^{-1}$, the iocation of the maximun shifting to slightiy larger values of $q$ as the beam energy increases. As was seen in the data for the $2_{1}^{+} ; 0$ state, the anaiyzing power at $\varepsilon_{p}=398 \mathrm{MeV}$ is dropping more rapidiy than the data at $E_{p}=597$ or $698 \mathrm{MeV}$. Indeed, $A_{y}$ is seen to have the same value at $9-1.8 \mathrm{Em}^{-1}$ for ali beam energles.

The larger error bars on the data points for smalier $v a l u e s$ of $q$ reflect poor statistics. In this region this state is relatively weakly exclted with respect to other states in the spectrum, and consequently obtaining satisfactory statisties for the more prominent states demandso that the statistics for more weakiy excited states be necassartly poorer. 
The data for o*A show behaviour similar to that for the $2_{1}^{+} ; \theta$ and $\theta_{2}^{+} ; \theta$ stares discussed previousiy. There $1 s$ a strong peak at $9-1.3 \mathrm{fm}^{-1}$ for alj energies: the smail shifts in opposite directlons for maxima in $\sigma$ and

${ }^{A} y$ as a Eunction of $E_{p}$ appear to compensate when the product is taken. The data for $E_{p}=597$ and 698 MeV are almost identical, and much larger than those for $E_{p}=398 \mathrm{MeV}$. The ratios taken at the vaiue of $q$ for which the data is a maximum are 2.2:2.2:1.0, normailzed to the value for $E_{p}=398$ MeV. We note that the absence of difference between data for the two higher beam energies is not in agreement with what 1 s seen for the $2_{1}^{+} ; \theta$ and $\theta_{2}^{+} ; \theta$ states.

(e) The 1; 10 state at $E_{x}=10.84 \mathrm{MeV}$.

The differential cross section data are presented in Figure V-16, the anaiyzing power data in Figure V-17, and the $\sigma A_{y}$ data, together with common plots of $\frac{d \sigma}{d \Omega}$ and $A_{Y}$ in Figure $V-18$.

The data for this state at $E_{p}=597$ and $698 \mathrm{MeV}$ are truncated because of interference from the MBD cut as described in Chapter III. The probiem is especlaijy severe Eor $E_{p}=698 \mathrm{MeV}$ where oniy two iow momentum transfer data points are avaliabie. Consequentiy, meaningful comparisons can only be made for the data at $E_{p}=398$ and $597 \mathrm{MeV}$.

We find that the differential cross section data ex- 
$E_{x}=10.84 \mathrm{MeV} \mathrm{I}_{1}^{-} ; 0-$ Differential Cross Sections
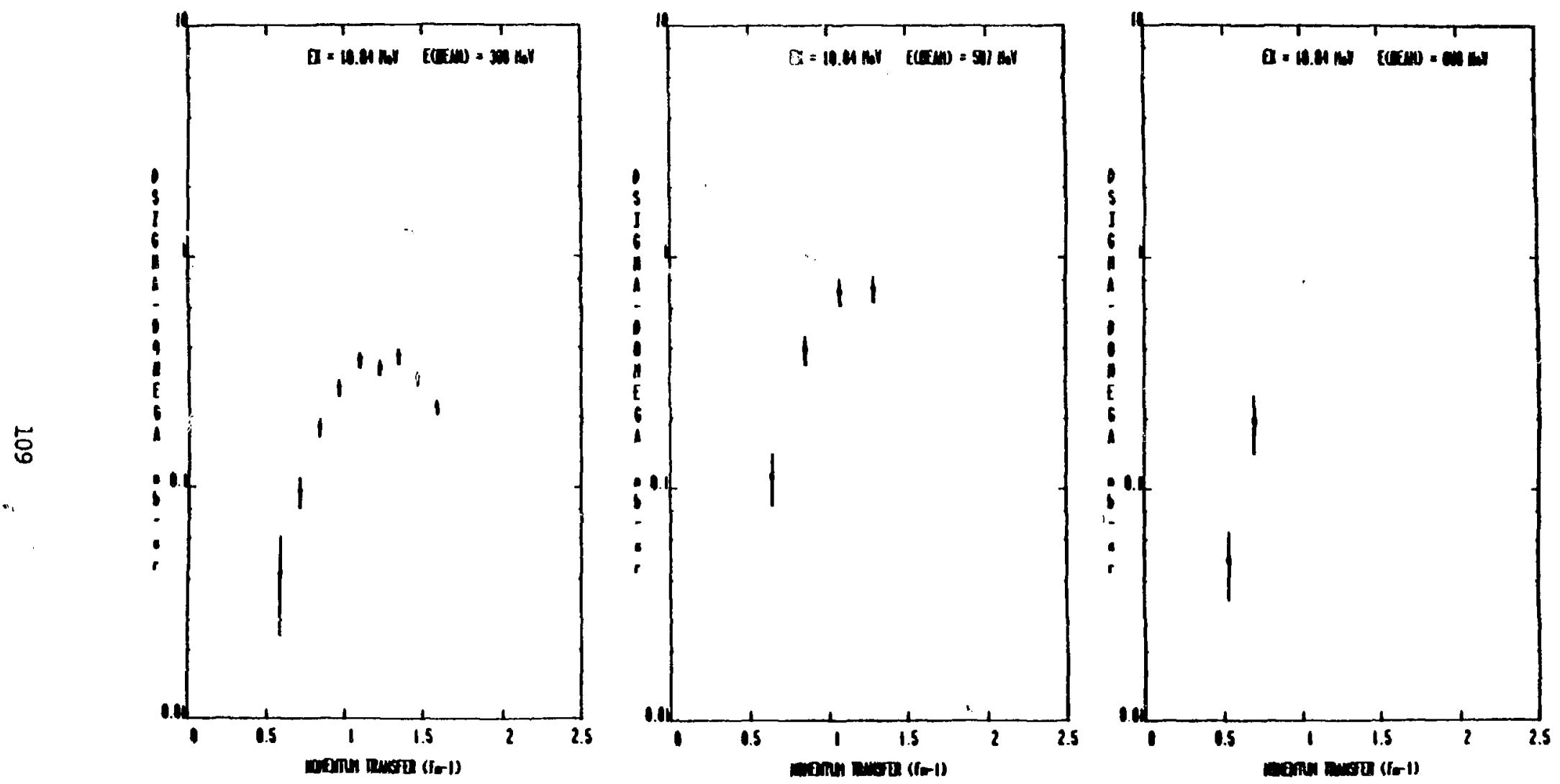

Figure $\mathrm{V}-17$. 

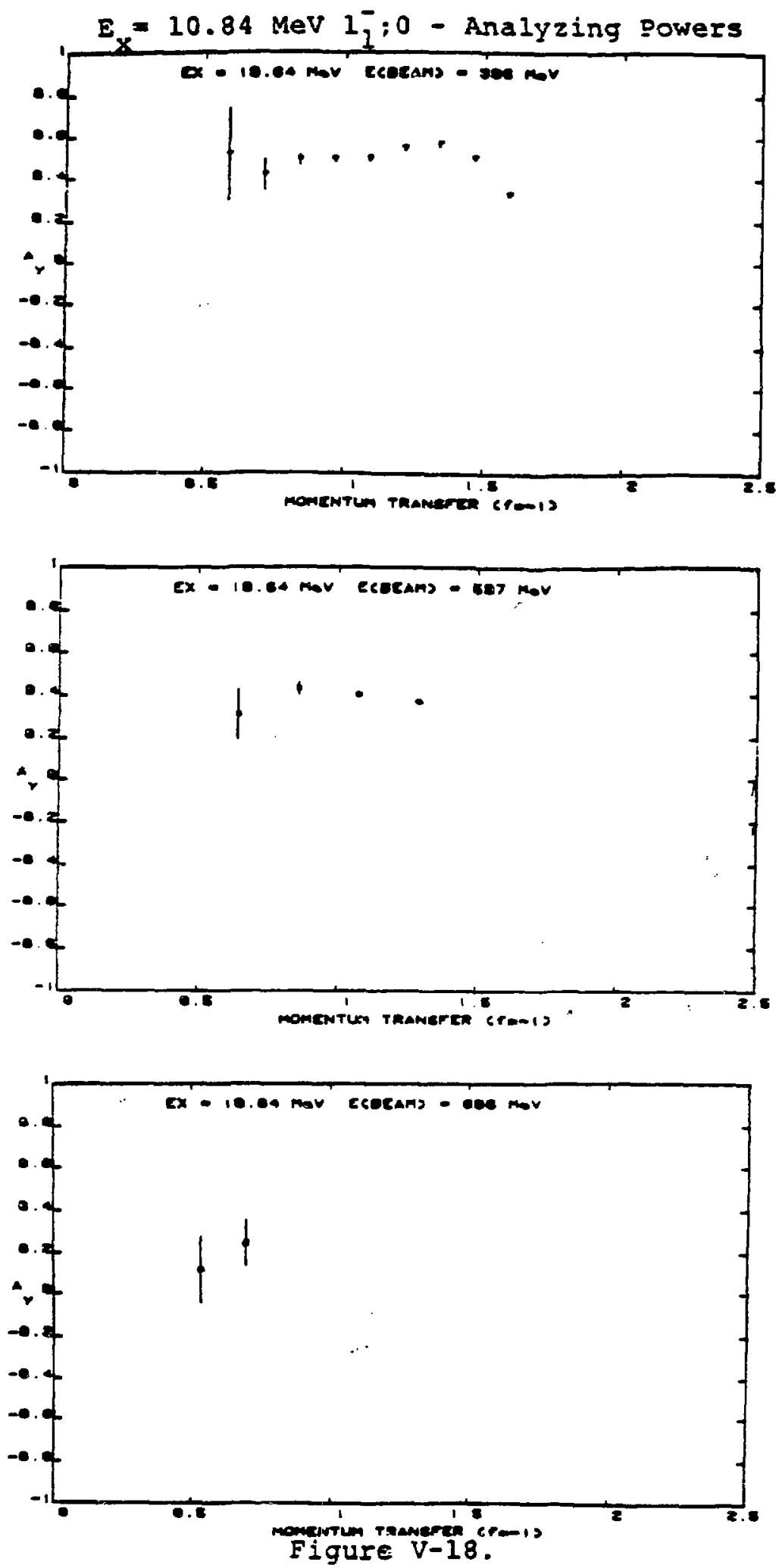
$E_{x}=10.34 \mathrm{MeV} 1_{1}^{-} ; 0$ - Comparative Observables
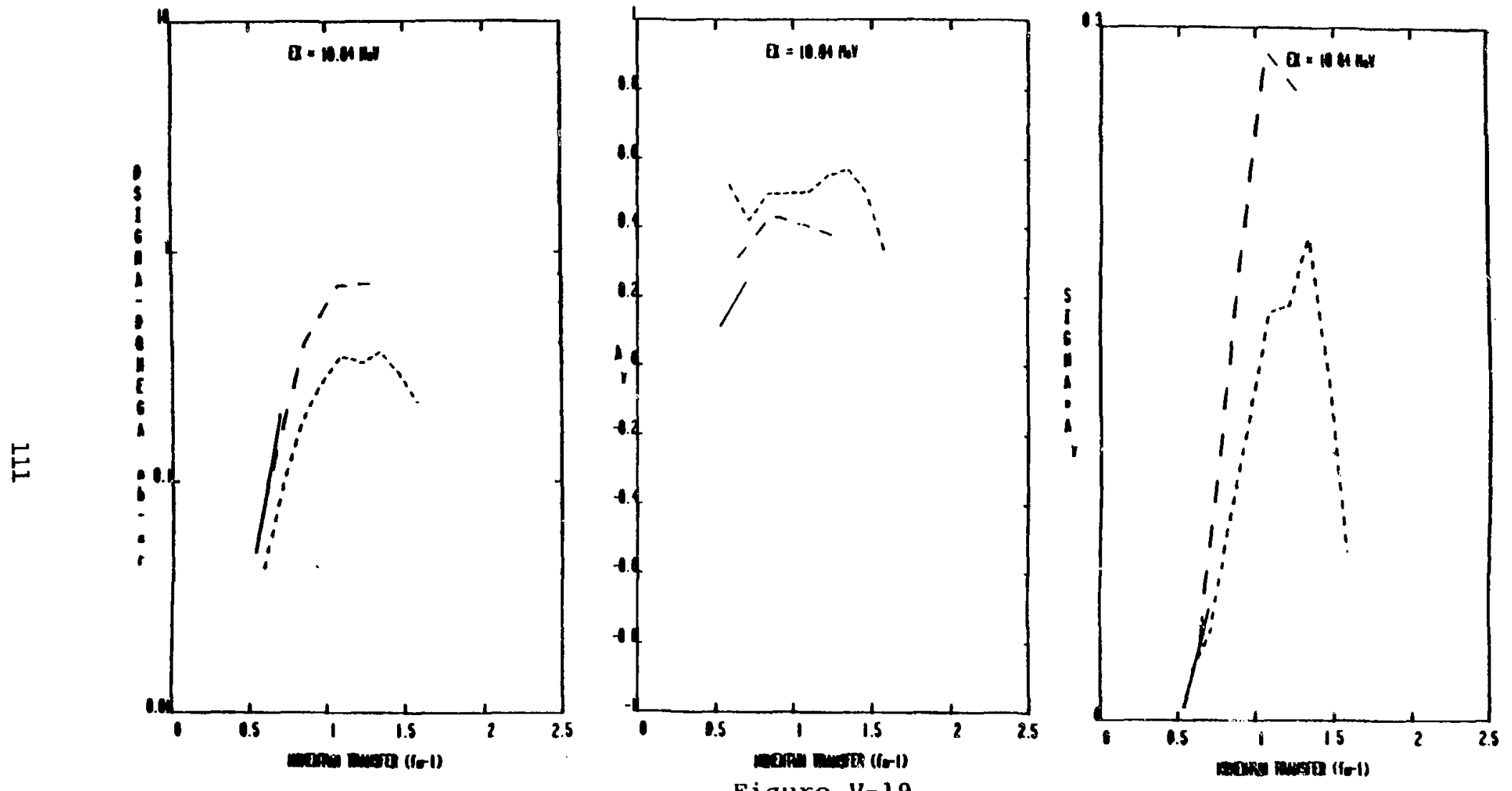
hlbit a smooth rise to a maximum value at $q=1.2 \mathrm{fm}^{-1}$ for both tiese bean energies. The ratio of the maxima at this value of $q$ is $2.0: 1.0$. No other significant observations can be made.

The andiyzing powers are uniformiy positive at all energies and exhibit little structure. The data for $\Sigma_{p}=$ $398 \mathrm{MeV}$ start to fall off towards 0 at $9-1.4 \mathrm{fm}^{-1}$, corresponding to the decrease in $\sigma$ from its maximum. The values of $A_{y}$ for $E_{p}=597 \mathrm{MeV}$ are uniformil smailer than those for $E_{p}=398 \mathrm{MeV}$.

The product $\sigma{ }^{*} A_{y}$ exhibits a structure similar to other states in this group already discussed. A sharp rise from - 0 at $q=0.5 \mathrm{fm}^{-1}$ peaks at $q=1.2 \mathrm{fm}^{-1}$, the data for $E_{p}=597 \mathrm{MeV}$ being greater than those for $E_{p}=398 \mathrm{MeV}$ at the maximum by a factor of 1.4 .

(f) The $4_{1}^{+}: 0$ state at $E_{x}=14.08 \mathrm{MeV}$

This is the last of the reii-known states in this category visibie in the spectra. It is a broad state, having an intrinsic width of some $260 \mathrm{keV}$ FWH. In addition, 1t lles on top of the large background due to the wide collective quadrupoie state at $E_{x}=15.3 \mathrm{MeV}$. These considerations require careful fitting of the background, and the large error bars for the high q data primarily reflect errors in background subtraction. The errors assigned to the 
$E_{x}=14.03 \operatorname{MeV} 4_{1}^{+} ; 0-$ Differential cross Sections
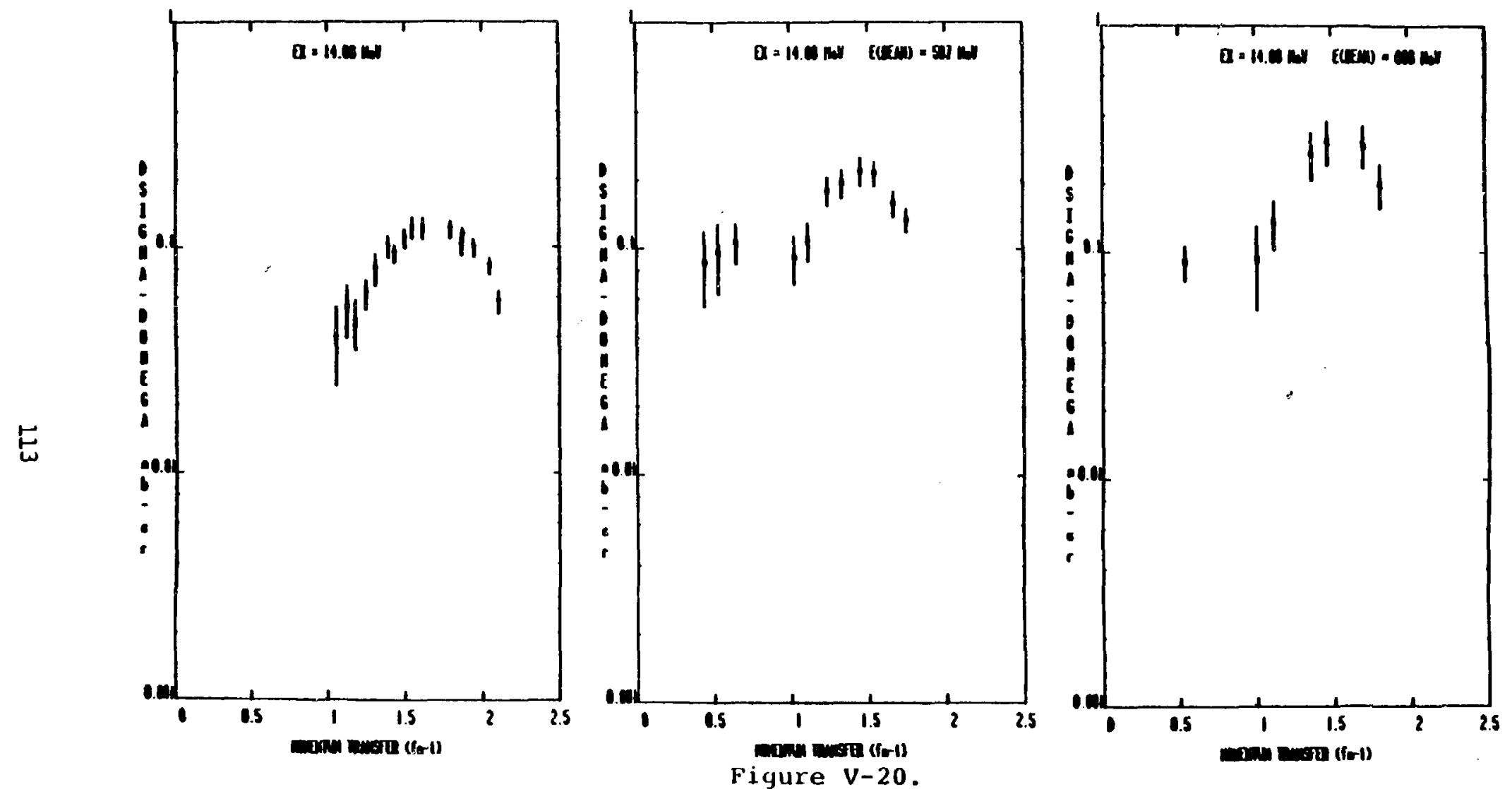

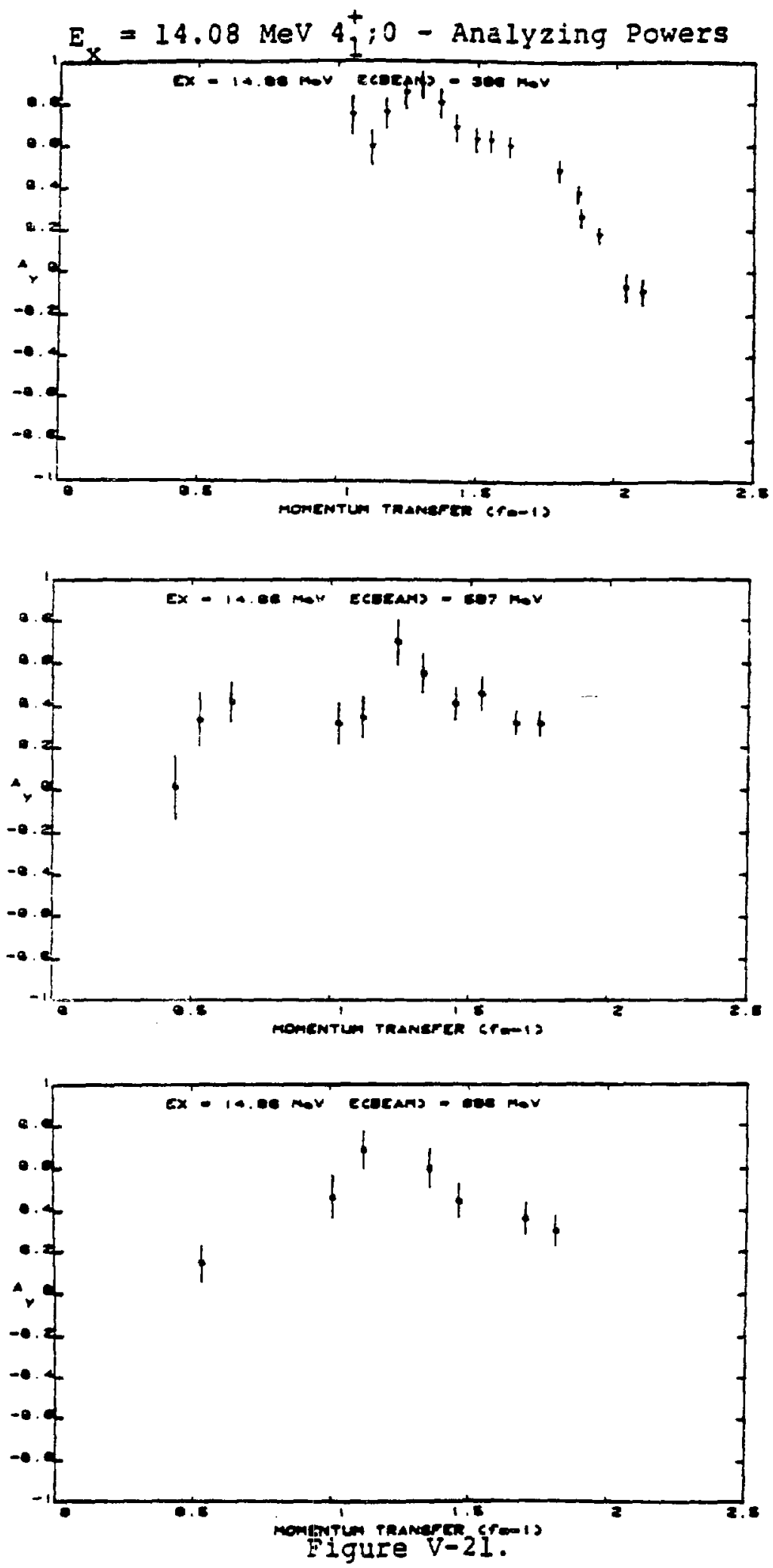


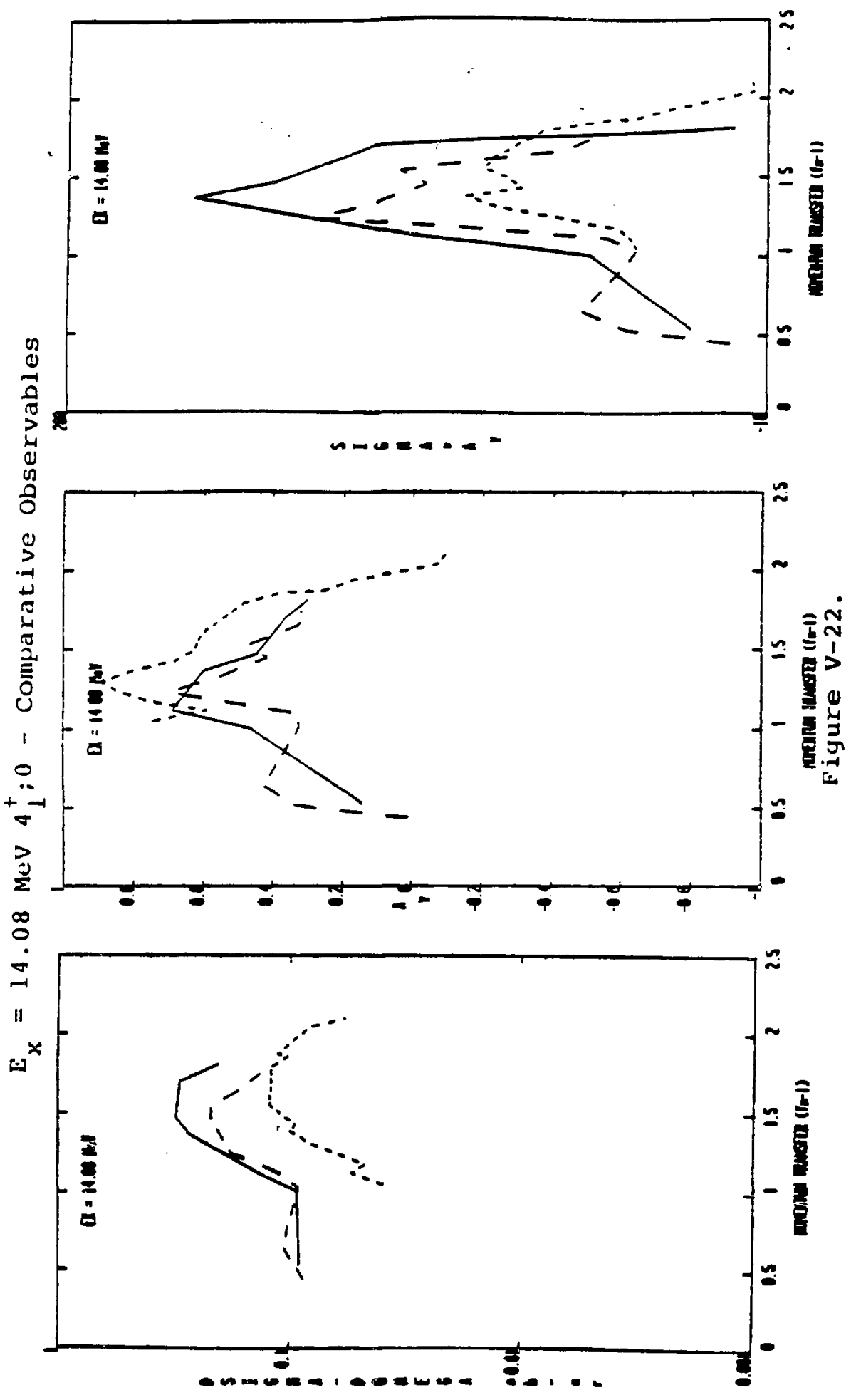


data at Iow $q$ are primarily statistical, the background beIng smoother in this region and the peaks being less welldefined than in the high q region.

The data are presented in Figures $V-20$ to $V-22$. The differential cross sections are seen to peak in the region $q$ $=1.5-1.7 \varepsilon^{-1}$, with a shlft to lower $q$ being evident as the beam energy rises. The data exhibit a minimum in the $q=$ $1.0 \mathrm{fm}^{-1} \mathrm{region}$, and in some cases it was possible to extract data in the $q=0.5 \mathrm{fm}^{-1}$ region. We note that this couid not be done at $E_{p}=398 \mathrm{MeV}$ where statistics were ver: poor. The ratio of differential cross sections at the main peak to the data at $q=0.5 \mathrm{fm}^{-1}$ for the two higher bean onergies is approximateiy 3.0. The ratio of the values of at the primary maximum are $2.6: 1.8: 1.0$.

The analyzing powers are generaily positive and atZ

tain the largest vaiues observed for this category of states. The general trend of iarger values for iower beam energies is apparrent, as is the more rapid fali-off at iarger $q$ for $E_{p}=398 \mathrm{MeV}$ where some negative values are found.

The product $\sigma * A_{y}$ shows strong peaking in the $q=$ $1.4 \mathrm{fm}^{-1}$ reglon, with the ratios of the maxima being $2.0: 1.6: 1.0$.

2) Isovector states of natural partty 
Only one well-known state with $\pi=(-1)^{L}$ and 1 sospin transfer $\Delta T=1$ is present in the spectrum. This is the $2_{1}^{+} ; 1$ state at $E_{x}=16.11 \mathrm{MeV}$. Data for this transition are presented in Figures $V-23$ to $V-25$.

In generai, statistics for this state were poor at forward angles (smail q) becuase it is weakly exclted in a spectrum dominated by strong natural parlty isoscaiar transitions and jow $\Delta L$ urnatural parity states. For large values of $q$ the differentlal cross section is seen to drop to the 1 microbern range, and agaln poor statistics dominate the uncertainties.

Examination of the differential cross section data as presented in Flgure $V-23$ reveals some interesting phenomena. A maximum in the cross- section is seen at a momentum transfer of $q=0.95 \mathrm{fm}^{-1}$ at ali beam energies. The shape of the cross section is not as symmetric as those obserbed for the isoscaiar states discussed previousiy le.g. the $\theta_{2}^{+} ; \theta$ and $\left.3_{1}^{-} ; \theta\right)$. Rather, the rate of decrease for $q$ > 1. $0 \mathrm{fm}^{-1}$ is less pronounced than the rise to the maximum at low 9 . Cleariy the most interesting feature of the data is not the shape nor magnitude of the differential cross section for a given beam energy, but rather the independence of the shape and magnitude of the data as a function of the incident beam energy. This is most clearly seen in flgure V25 where the three curves representing the data are seen to lie on top of one another. Such behaviour is markedly dif- 
$E_{x}=16.11 \mathrm{MeV} 2{ }_{1}^{1} 1$ - Differential Cross Sections
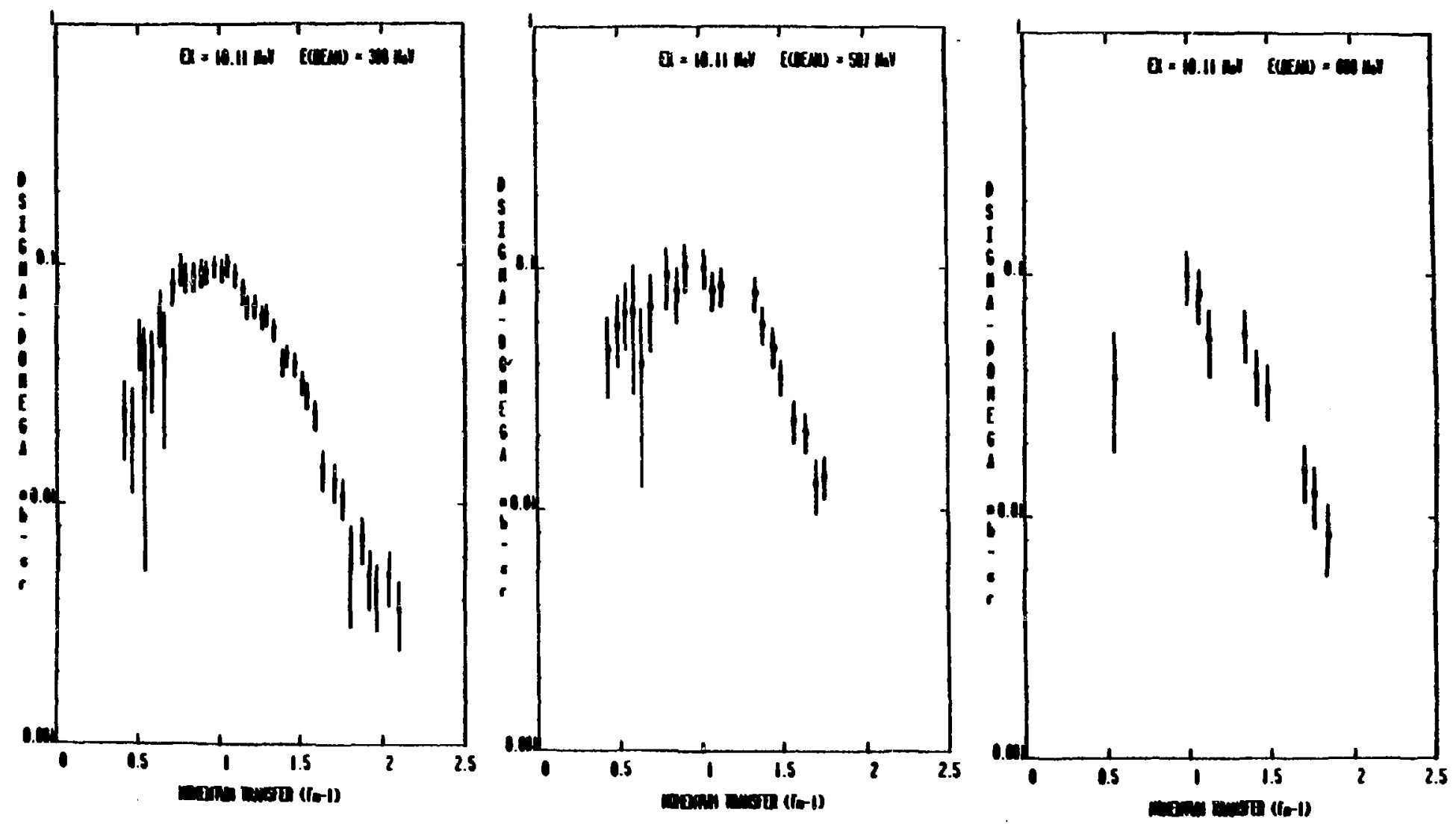

Figure V-23. 

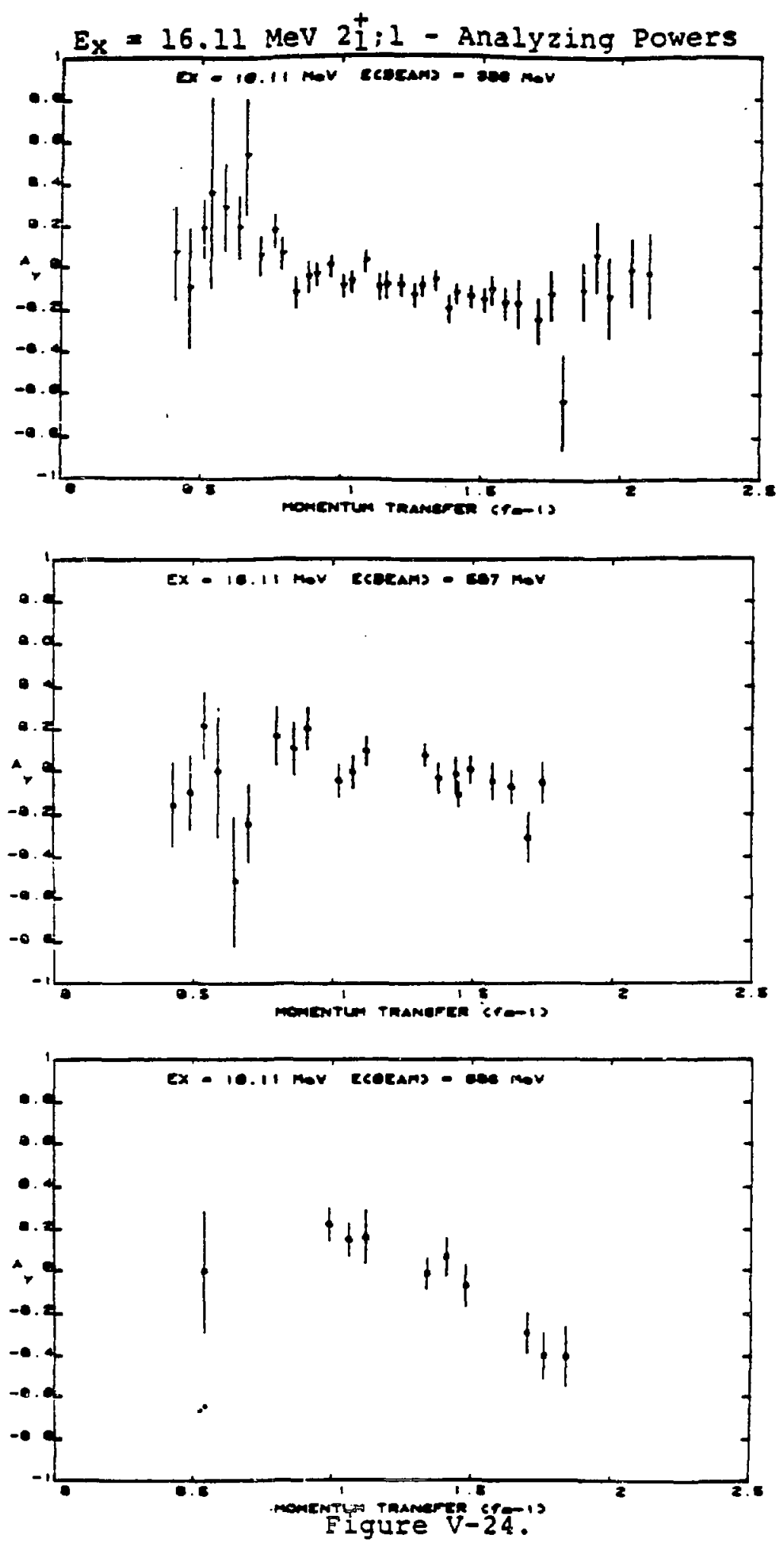


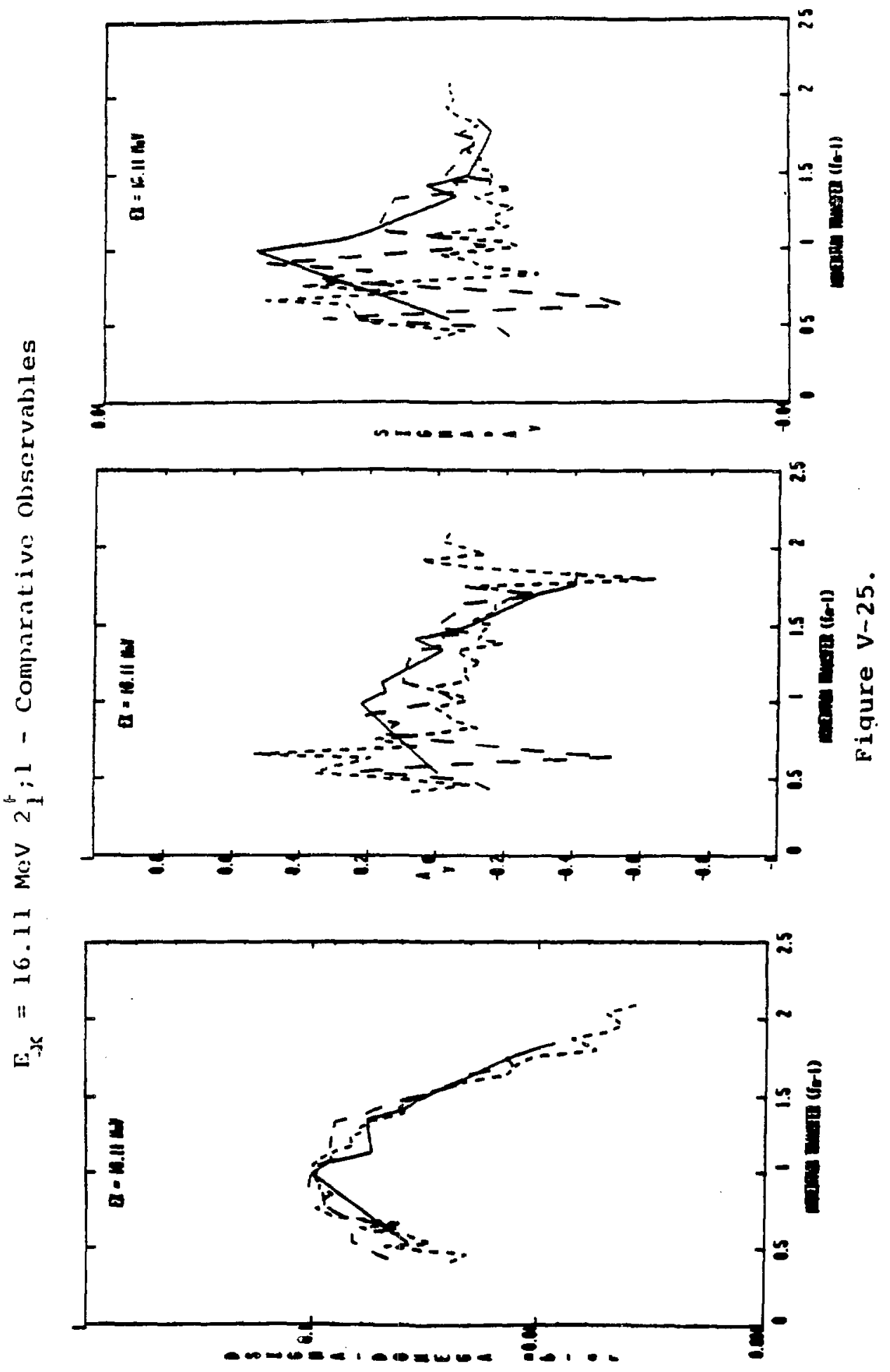


ferent from the previously discussed isoscalar transitions.

The analyzing power data, presented in Flgure V-24, show reasonable consistency of structure for all incident particie energies. For momentum transfers up to $q=1.5$ $\mathrm{fm}^{-1}$ there is a scatter of points around $A_{y}=0$, showing a trend from sightly positive values for small a to slightly negative values for larger $q$. For $E_{p}=398 \mathrm{MeV}$ the data, after dropping to slightly negative values for $1.5 \mathrm{Ea}^{-1} \leq q$ $\leq 1.75 \mathrm{fm}^{-1}$ return to values consistent with zero for $q>$ $1.75 \mathrm{fm}^{-1}$. The data for $E_{p}=698 \mathrm{MeV}$ show a continuing trend to negative values at $q=1.75 \mathrm{fm}^{-1}$ as do the data for $E_{p}=597 \mathrm{MeV}$.

The product $\sigma *^{*}$ shows large oscillations around zero for values of $q$ less than $1 \mathrm{fm}^{-1}$. The osclilations decrease as $q$ lncreases, ali curves converging to zero. This behaviour is an artifact of the osciliations of sign for $A_{y}$ for iow momentum transfers, coupied whth the drof to smail vaiues of the differentiai cross section for iarge values of q.

\section{3) Isoscaiar states of unnatural parity}

We present in this section data for states having no isospin transfer $(\angle T=0)$ which are primarily populated through spin transfer mechanisms ( $L S=1$ ) resulting in parities $\pi=(-1)^{L+1}$. Several such states are known to exist in 
${ }^{12} \mathrm{C}$, the most well-known belng the $1_{1}^{+}: 0$ at $E_{x}=12.71 \mathrm{Mev}$. In addition, $2^{-} ; 0$ states are known to exist at $E_{x}=11.83$ and $13.35 \mathrm{MeV}$.

(a) The $1_{1}^{+} ; 0$ state at $E_{x}=12.71 \mathrm{MeV}$

The differentiai cross section data are presented in Figure $v-26$, the analyzing power data in Figure $v-27$ and the product $\sigma^{* A}$ together with common piots of the data in Figure $v-28$.

The differential cross section is seen to attaln a maximun value of $0.26 \mathrm{mb} / \mathrm{st}$ for $E_{p}=398 \mathrm{MeV}, 0.38 \mathrm{mb} / \mathrm{sr}$ for $E_{p}=597 \mathrm{MeV}$ and $0.33 \mathrm{mb} / \mathrm{sr}$ for $E_{p}=698 \mathrm{MeV}$ at the smajlest vaiues of $q$ for which data were taken. It appears that the data are flattening out as $q$ is extrapolated to zero, and it may be appropriate therefore to interpret the zero momentum transfer cross sections as being very close to these values. The data are seen to drop smoothiy by an order of magnitude to a momentum transfer of about $1.4 \mathrm{fm}^{-1}$, where a smail shouider in the data is evident. This shouider is seen at ail beam energies. At $E_{p}=398 \mathrm{MeV}$, where data exist for $q$ up to $2.2 \mathrm{fm}^{-1}$, the data are seen to continue to drop after passing through the shoulder, but not as steeply as is observed in the lower momentum transfer region. Of speclal interest is the observation that the cross section is, in general, almost constant in shape and magnitude as a function of incident beam energy. This is most easily seen in 
$E_{x}=12.71 \mathrm{MeV} 1_{1}^{+t} ; 0$ - Differential Cross sections
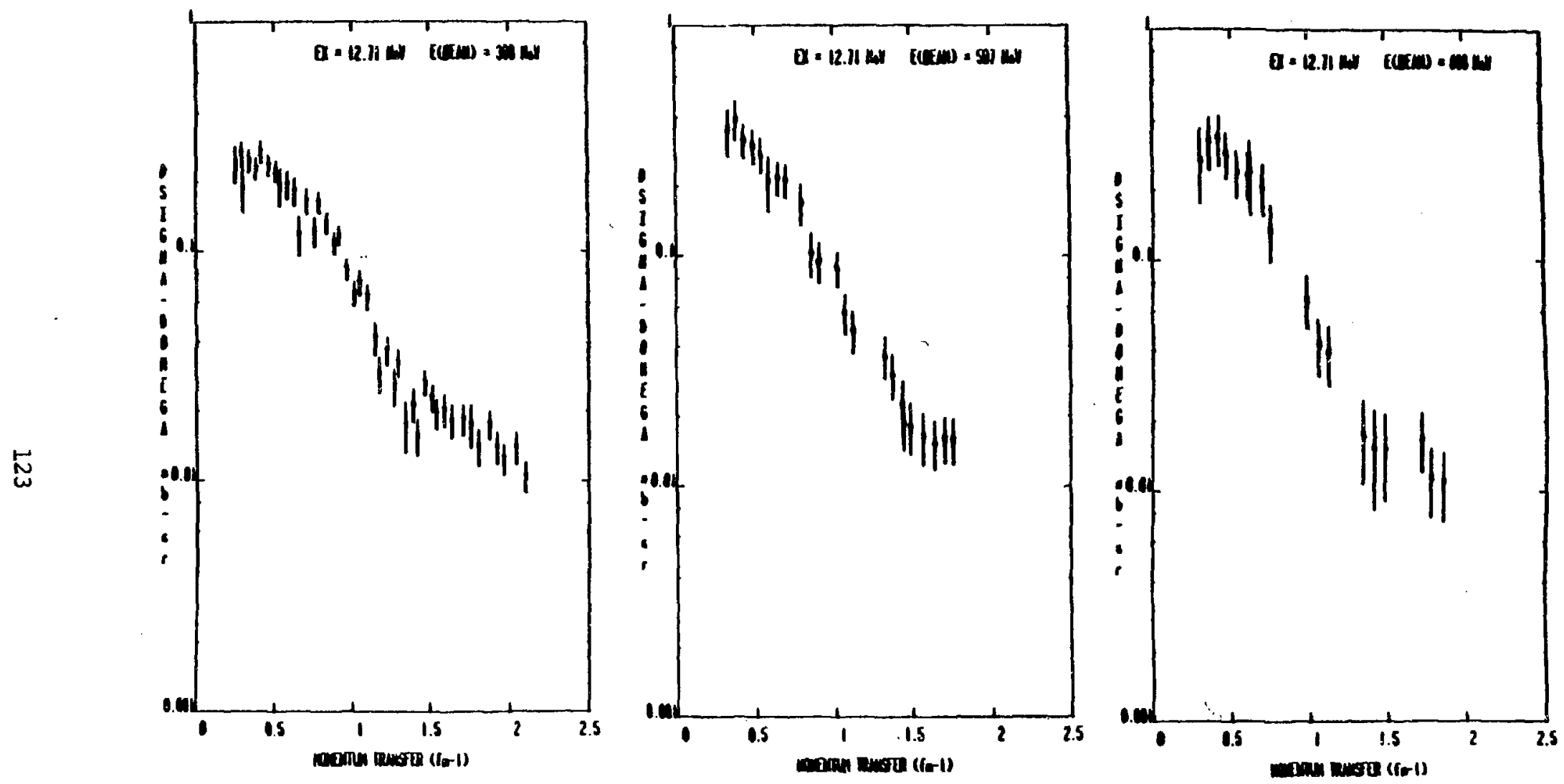

Fiqure V-26. 

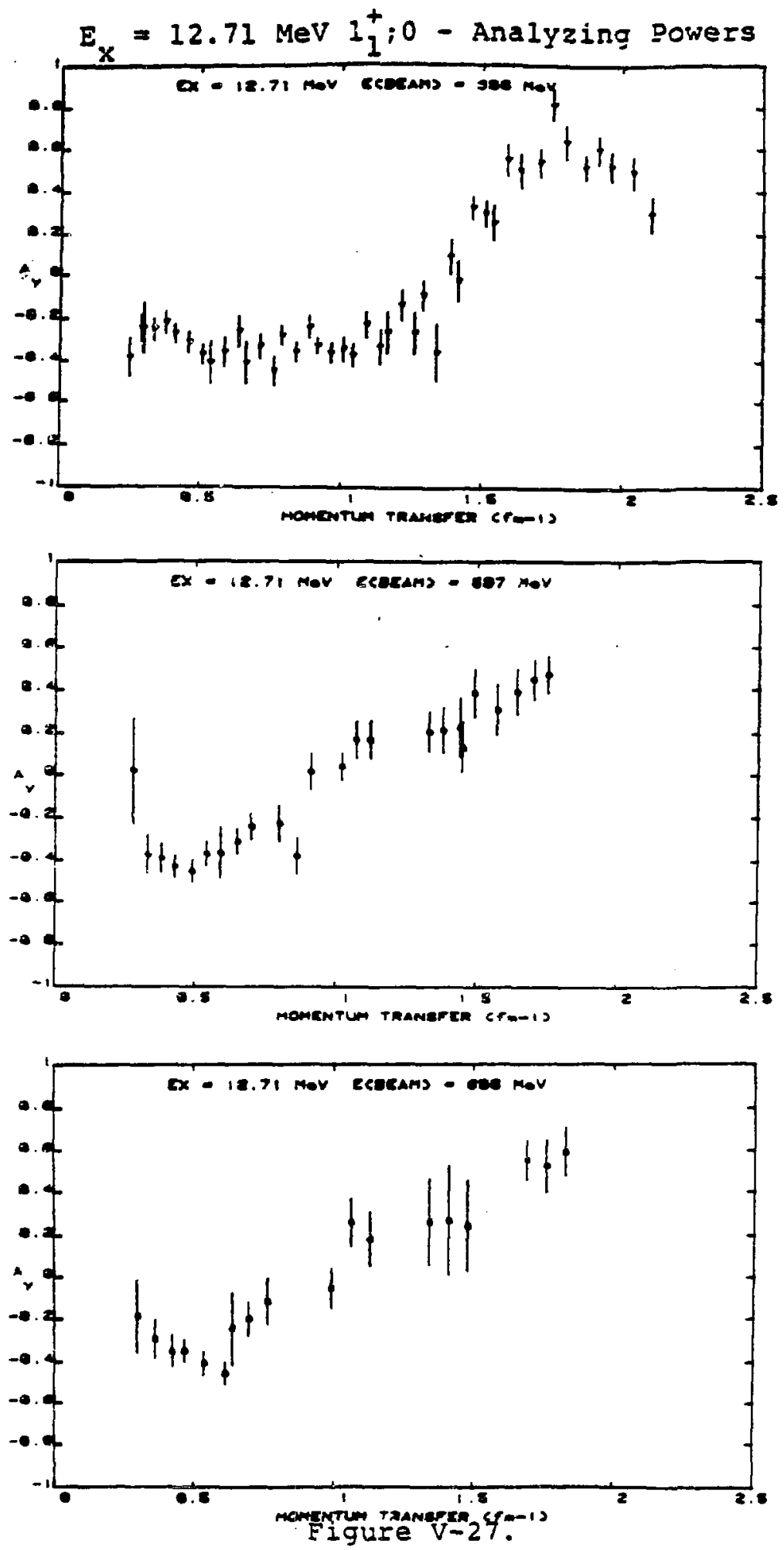
$\mathrm{E}_{\mathrm{x}}=21.71 \mathrm{MeV} 1_{1}^{+} ; 0$ - Comparative Observables
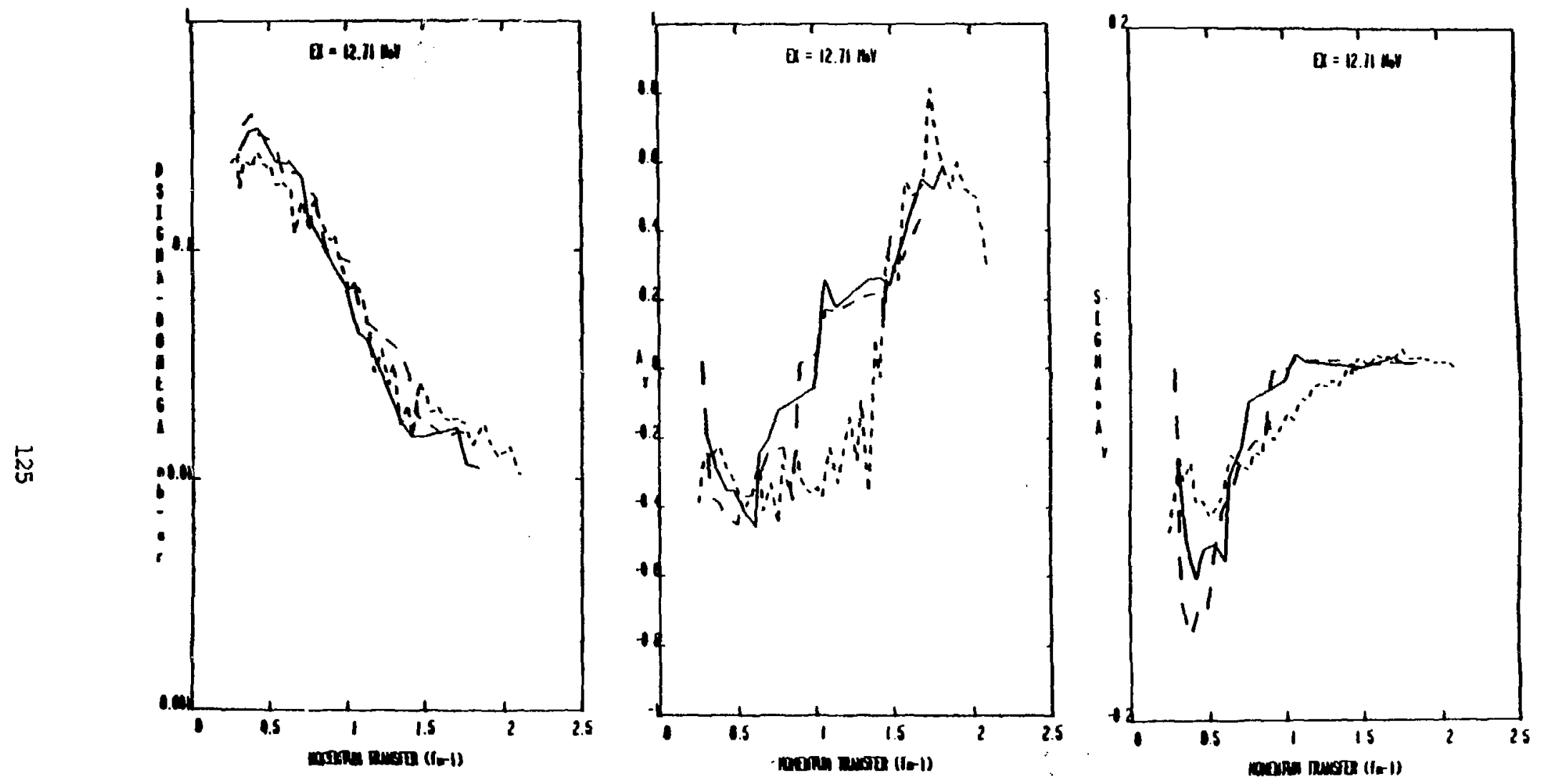

Figure $\mathrm{V}-28$. 
Elgure $V-28$.

Examination of Flgure $V-27$ yleids interesting observations for the anaiyzing power. In contrast with aij data presented thus Ear, iarge negative anaiyzing powers $1-0.3$ to -0.4$)$ are seen for smail values of $q$. For $q \leq 0.5 \mathrm{fm}^{-1}$ the data for aI are the data for $q \geq 1.5 \mathrm{fm}^{-1}$ where $A_{y}$ is strongly positive.

The intermediate region of $0.5 \mathrm{fm}^{-1} \leq q \leq 1.5 \mathrm{fm}^{-1}$ shows some differences from beam energy to beam energy. For $E_{p}=398 \mathrm{HeV}$, where the quality of the data is best, the vaiue of $q$ for which the sign of $A_{y}$ changes from nogative to positive is about $1.4 \mathrm{fm}^{-1}$, coinciding with that at which the shouider is observed in the differential cross section. For $E_{p}=597 \mathrm{MeV}$, the cross-over point has shifted to about $1 \mathrm{fm}^{-1}$, as has that for $E_{p}=698 \mathrm{MeV}$. As such, these data then do not coincide with the shouider seen in the differential cross section data.

The produce $\sigma \star^{A} y$ shows interesting features. For $q<0.5 \mathrm{fm}^{-1}$ it is sharply negative reflecting the sign of $A_{Y}$. Differences in magnitude in this region are attributable to sightly different magnitudes for the cross section. The product then rises smoothly to small positive vaiues and then falls to zero as $q$ increases, the rise for $E_{p}=398 \mathrm{MeV}$ being less pronounced thar that for the higher beam energies. This is evidence for the shift to lower $q$ of the 
change in sign of $A_{y}$ for the higher beam energles together with the rapid decrease in the magnitude of the differentiai cross section.

The form of $A_{y}$ and $\sigma{ }^{*} A_{y}$ are so characteristic as to warrant the postuiate that such behavfour could be a signature for isoscalar unnatural parity transtions. This postuiate wit be examined in more detail in succeeding sections and the next chapter.

(b) The $2_{2}^{-} ; \sigma$ state at $E_{x}=13.35 \mathrm{MeV}$

Thie data for this state are presented in slgures $V-$ 29 to v-3i. This transition is very weakiy excited and is oniy visible in the spectra for vaiues of q greater than 1.4 $\mathrm{fm}^{-1}$. Consequentiy, very $\mathrm{fsw}$ data points are available and ittie detailed information may be obtained.

The differential cross sections shown in figure $V-29$ are seen to be of the order of $0.01 \mathrm{mb} / \mathrm{sr}$, and aimost constant in magnitude as a function of beam energy. The anaiyzing powers are positive in the region where data exist. The product $\sigma{ }^{* A} y$ does not yieid any usefui information.

4) Isovector states of unnatural parity.

This class of states is populated by transitions invoiving transfer of one unit of both spin and isospln ( $\Delta S=$ 
$E_{\mathbf{x}}=13.35 \mathrm{MeV} 2{ }_{2}^{-} ; 0$ - Differential Cross Sections
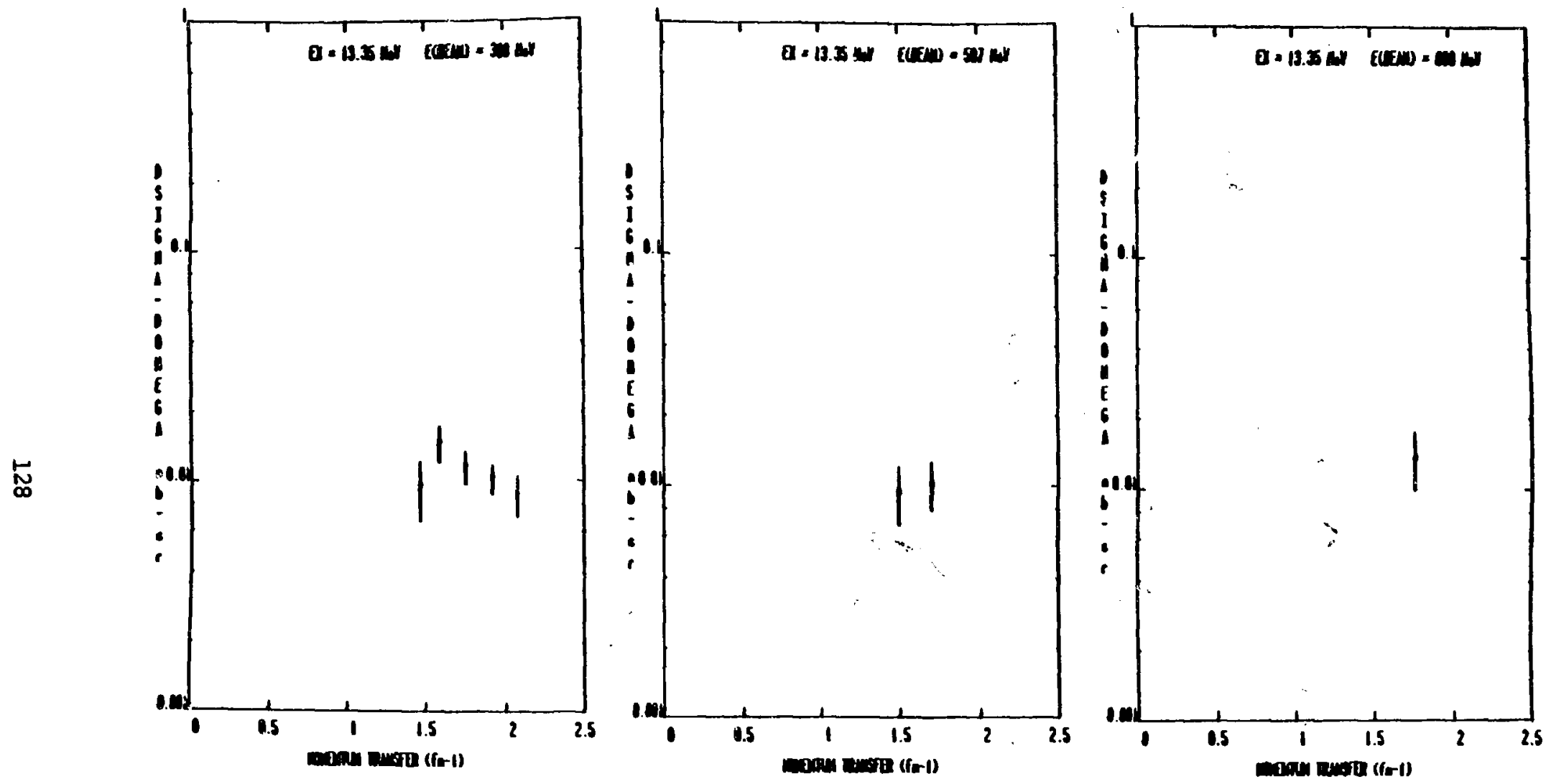

Figure V-29. 

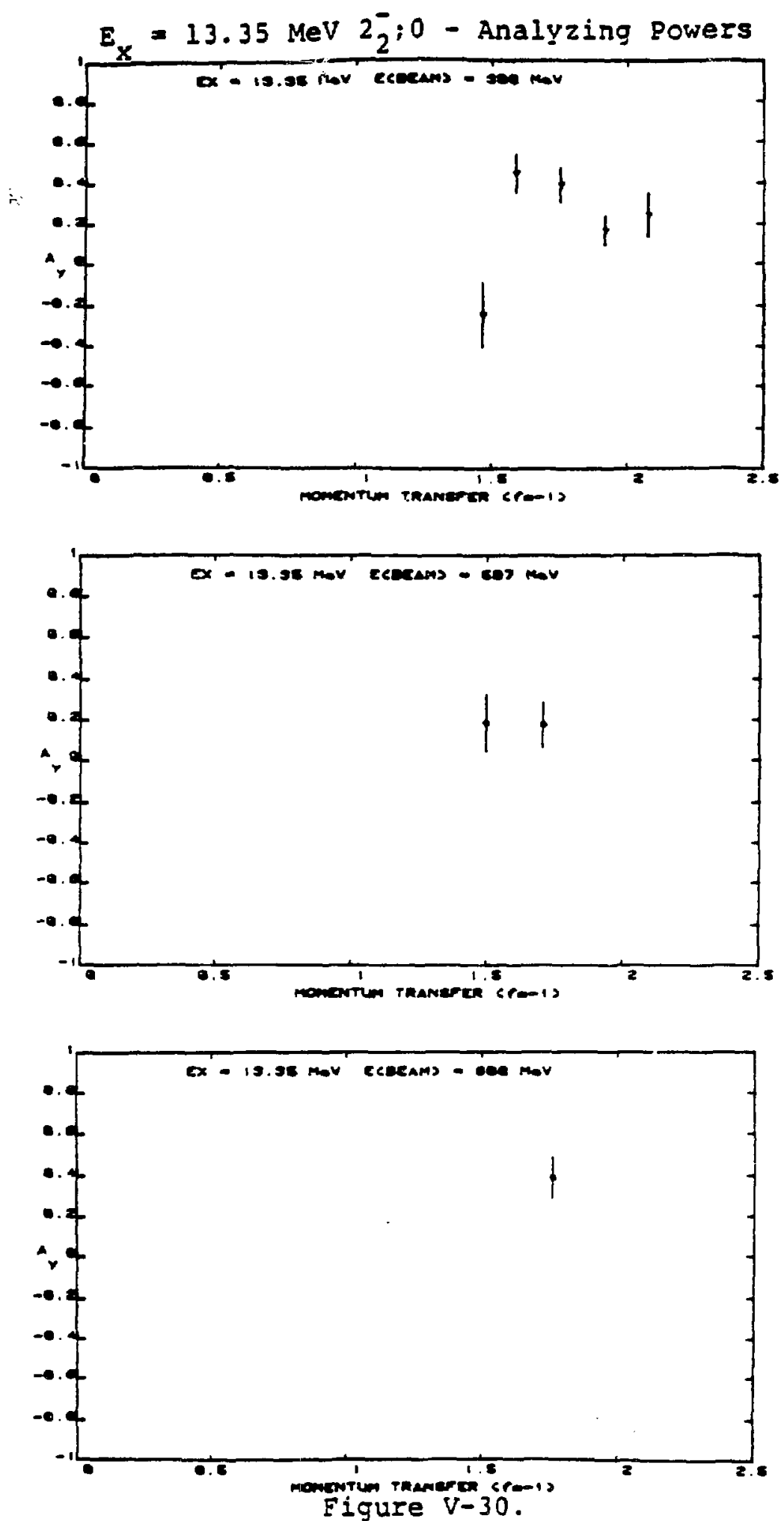
$E_{x}=13.35 \mathrm{MeV} 22_{2}^{-} ; 0$ - Comparative Ouservables
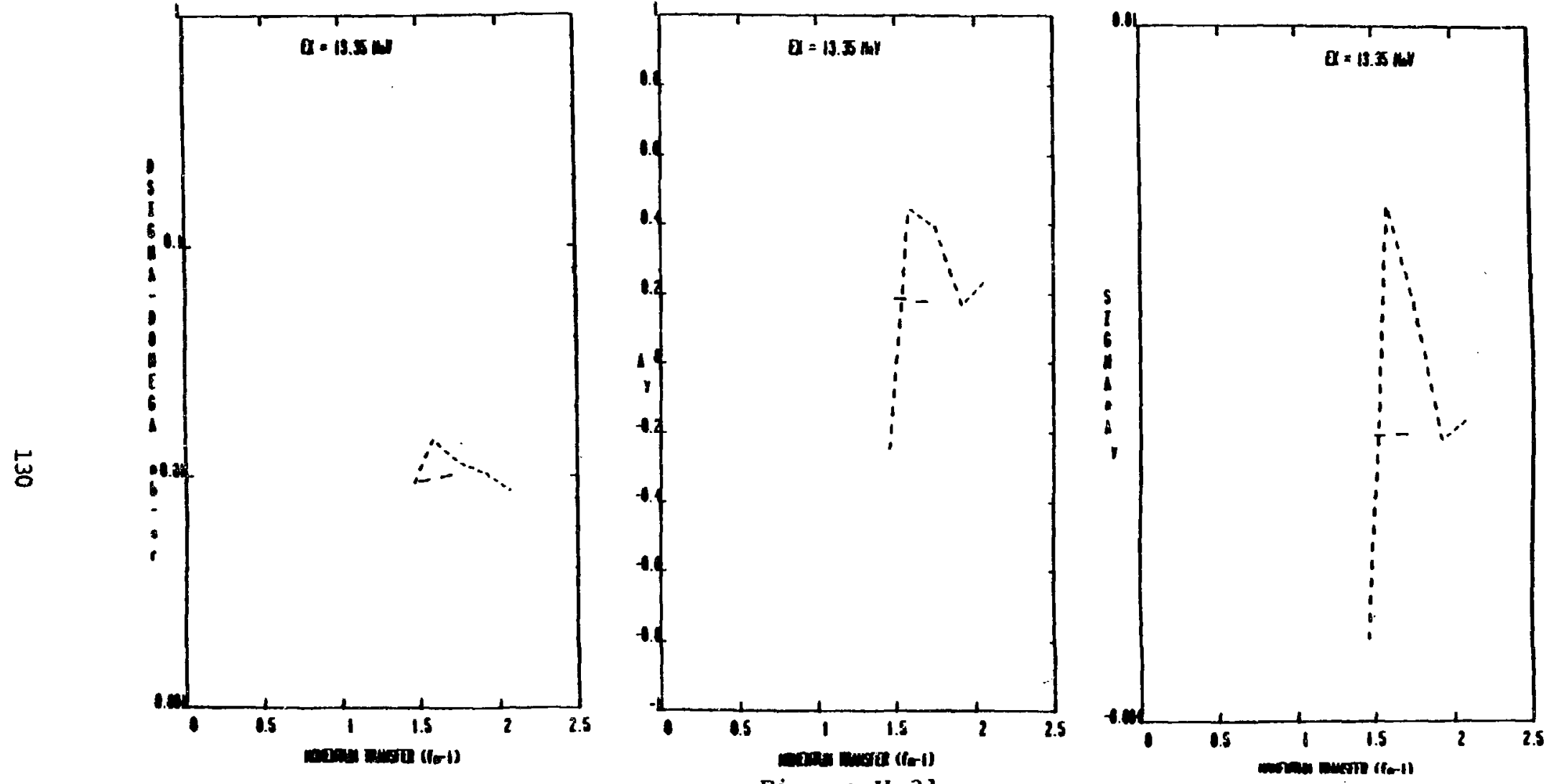

Figure v-31. 
1, $I=1)$. The best exampie of such a transition is the $I_{1}^{+}: 1$ state at $E_{x}=15.11 \mathrm{MeV}$. Also of interest is the $2 ; ; 1$ state at $E_{x}=16.58 \mathrm{MeV}$, the spin, parity and isospin assignments of these states being well-established.

(a) The $1_{1}^{+} ; 1$ state at $E_{x}=15.11 \mathrm{MeV}$

The differential cross section data are presented in Figure V-32, the analyzing power data in Figure $V-33$ and combined plots together with $\sigma \star^{A}$ in Figure V-34.

The differential cross section data show an almost exponential decrease over two orders of magnitude for the range $0.3 \mathrm{fm}^{-1}<\mathrm{q}<1.3 \mathrm{fm}^{-1}$. Some minor structure 1 s seen for $q-0.6 \mathrm{fm}^{-1}$ at all beam energies. The magnitude of the cross section for the different beam energies at $q-0.35$ $\mathrm{fm}^{-1}$ is constant and about $1.3 \mathrm{mb} / \mathrm{sr}$. A smail maximum, or 'shouider' is ciearly evident in the $E_{p}=398 \mathrm{MeV}$ data at $q$ $=1.6 \mathrm{fm}^{-1}$, and aithough the data for the higher beam energies is less complete, evidence for this structure is aiso present there. The increase in uncertainty in the data points as an increasing function of $q$ reflects poorer statistics coupled with a decreasing peak-to-background ratio. Uncertalnties at low q are small because this state, apart from the elastic scattering, is dominant in the spectra.

Again, the most remarkable feature of these data is the independence of the inagnitude and shape of the cross 
$E_{x}=15.11 \mathrm{MeV} 1_{1}^{+} ; 1-$ Differential Cross Sections
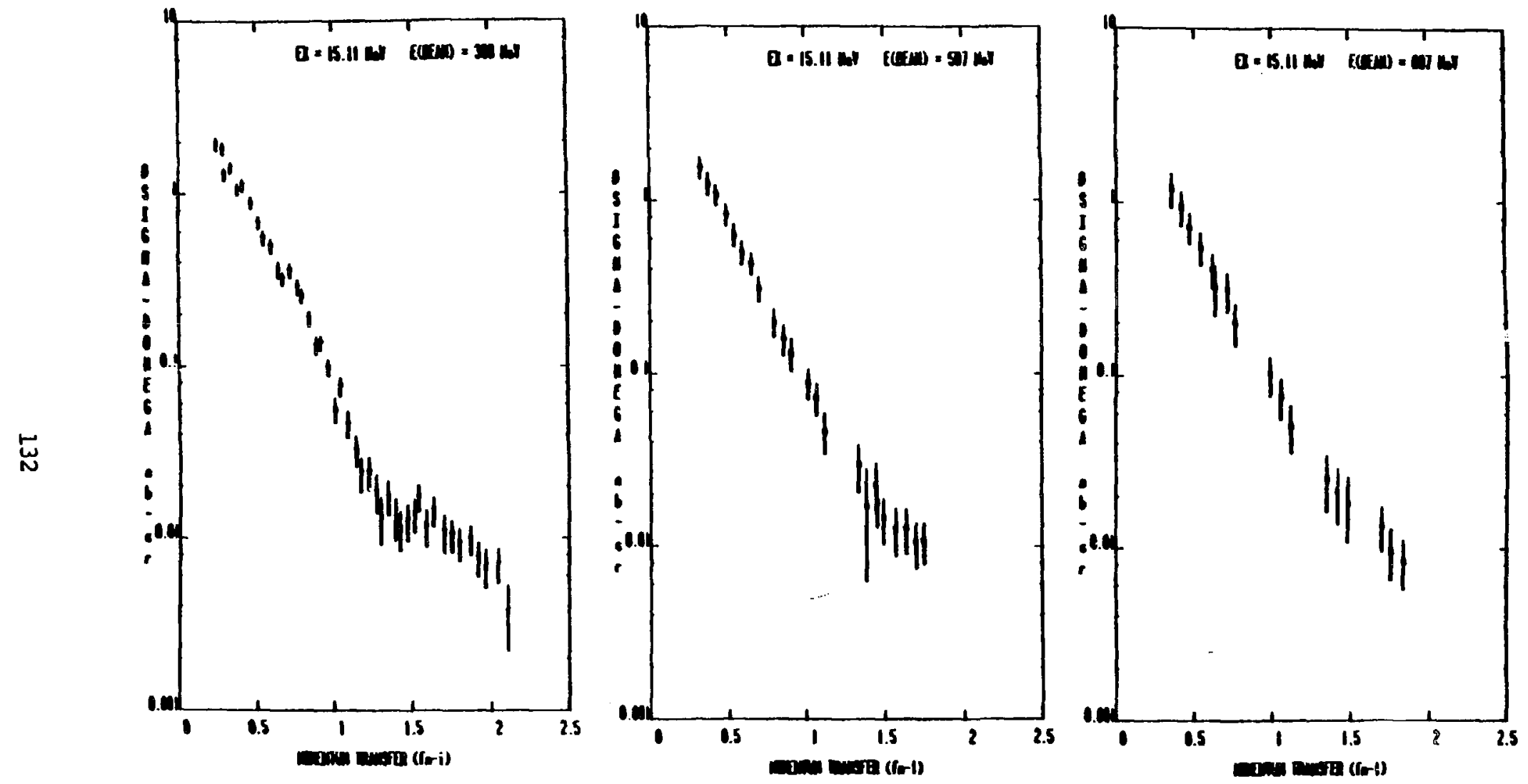

Figure $\mathrm{V}-32$. 

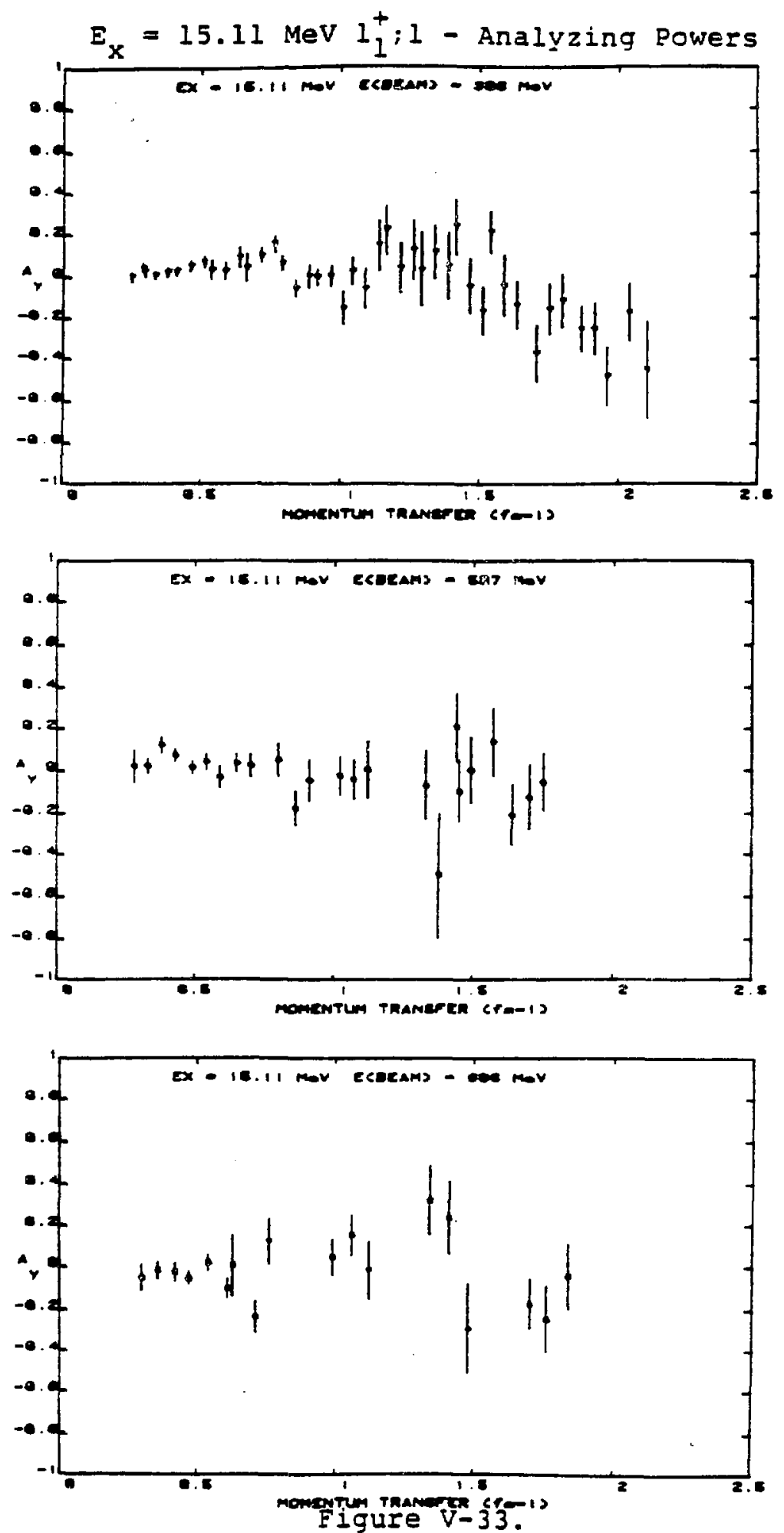

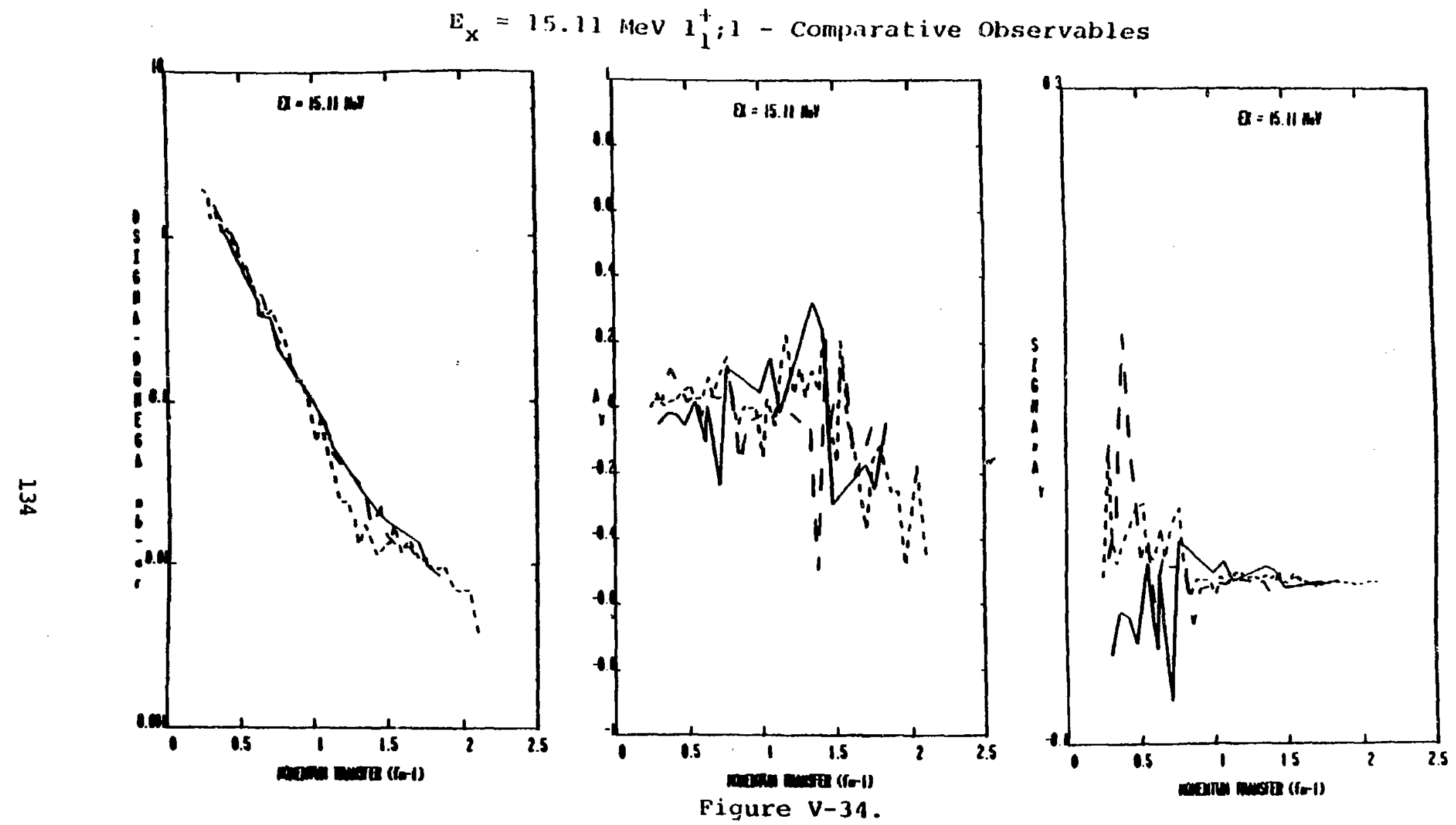
section as a function of incldent particle energy. There is some slight discrepancy in the $q=1.3 \mathrm{fm}^{-1} \mathrm{reg}$ ion where the $E_{p}=398 \mathrm{MeV}$ ata are silghtly lower than the data at $E_{p}=$ 597 or $698 \mathrm{MeV}$, but up to this point the equivaience is almost exact.

The analyzing power data in Figure $v-33$ show systematic features. For values of $q$ up to about $0.6 \mathrm{fm}^{-1}, A_{y}$ is consistent with zero. In the $q=0.7 \mathrm{fm}^{-1} \mathrm{reg}$. Ion a rise to smali positive values is seen, followed by a drop back to zero in the $q=1 \mathrm{fm}^{-1}$ region. Smalj positive values are attained in the $1.2 \mathrm{fm}^{-1} \leq \mathrm{q} \leq 1.5 \mathrm{fm}^{-1}$ region, after which a consistent trend toward negative vaiues is seen. These features are most evident for the $E_{p}=398 \mathrm{MeV}$ data, and are consistent with the data at $E_{p}=597$ and $698 \mathrm{MeV}$. The zero vaiue for $A_{y}$ at iow q shouid be contrasted with the strongiy negative vaiues seen for this observabie for the isoscalar state with the same spin an parity. It would appear that the behaviour of the analyzing power as a function of $q$ for unnatural parity states may therefore be a sensitive probe of the isospin transfer nature of different modes of excitation in the nucleus.

The product $\sigma{ }^{*} A_{Y}$ shows strong fluetuations in the Iow q region, representative of the fluctuations of $A_{y}$ about zero. This quantity converges rapidiy to zero as $q$ increases. this being an artifact of the exponentialiy decreasing differential cross section. 
(b) The $2_{1}^{-}$il state at $E_{x}=16.58 \mathrm{MeV}$

This state, with an intrinste width of some $300 \mathrm{keV}$ FWHM, is found to be populated at larger momentum transfers ( $\left.q \geq 1 . \sigma \mathrm{fm}^{-1}\right)$. The data for this Eransition are presented in Figures $V-35$ to $V-37$.

The differential cross section has a maxtmus for $q$ $1.35 \mathrm{fm}^{-1}$, and we note from the superrosed data in Figure $v-37$ and also the data points with error bars in Figure V-35 that, to within uncertainties, the magnitude and shape of the cross section are invariant with respect to incident projectile energy. The maximum vaiue attained by the cross section is $0.035 \mathrm{mb} / \mathrm{sr}$.

The analyzing powers showsi in Fiqure $V-36$ are un $1-$ formiy positive, and the data for the lower two beam energies are failing off slowiy as $q$ increases. For these lower beam energies there appears to be some structure in $A_{y}$ for vaiues of q close to the maximum in the differential cross section. Such an observation cannot be made for the data at $E_{p}=698 \mathrm{MeV}$ because of the large interval in $q$ between data points.

The product $\sigma * A_{y}$ shows structure somewhat resembiling the peaks seen for natural parlty states. However, if the uncertainties in this quantity are taken into account, such structure is washed o.jt and the curves piotted in Figure $\mathrm{V}-37$ are not inconsistent with one another as might 
$E_{x}=16.58 \mathrm{MeV} 2_{1}^{-} ; 1$ - Differential Cross Sections
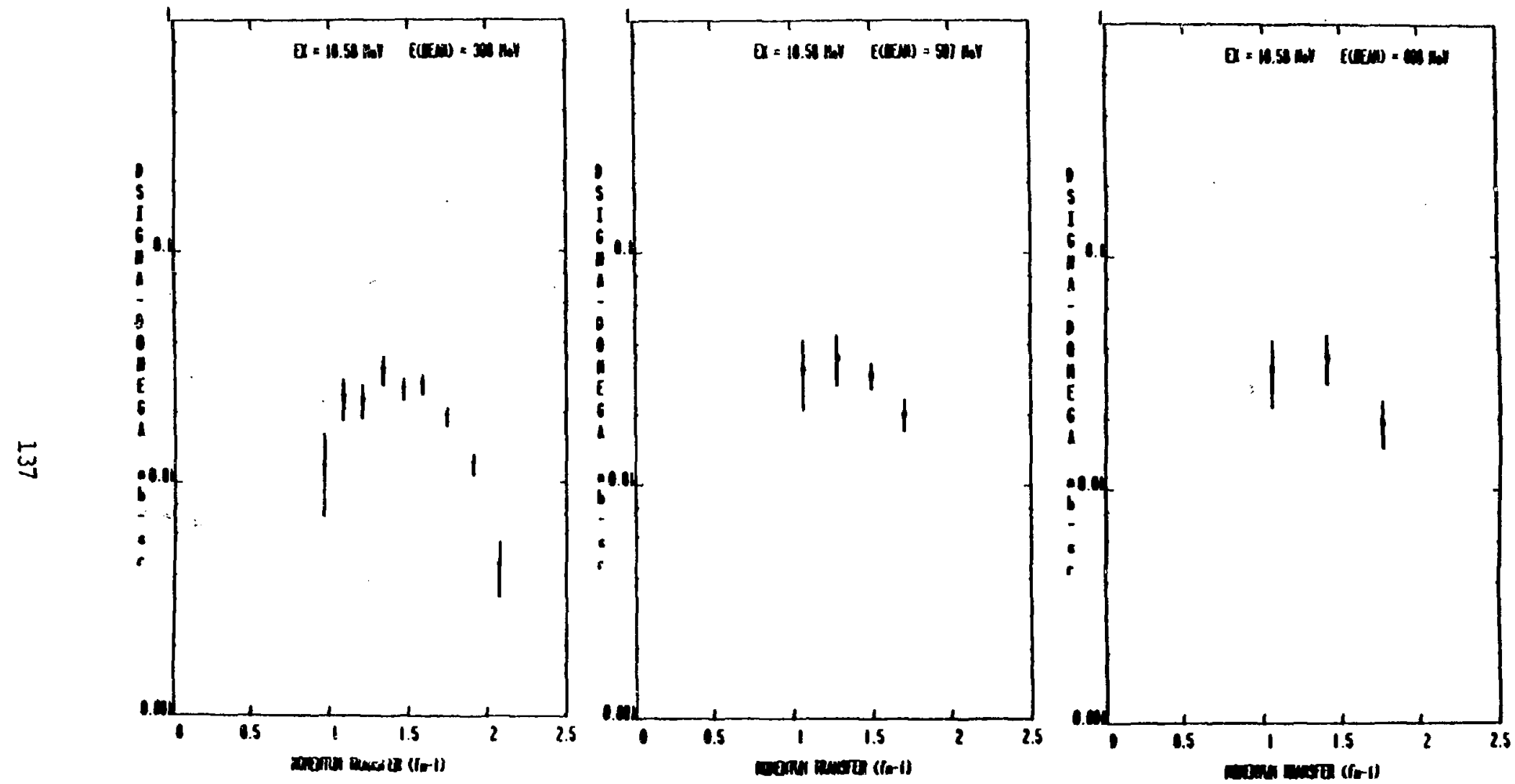

Figure V-35. 

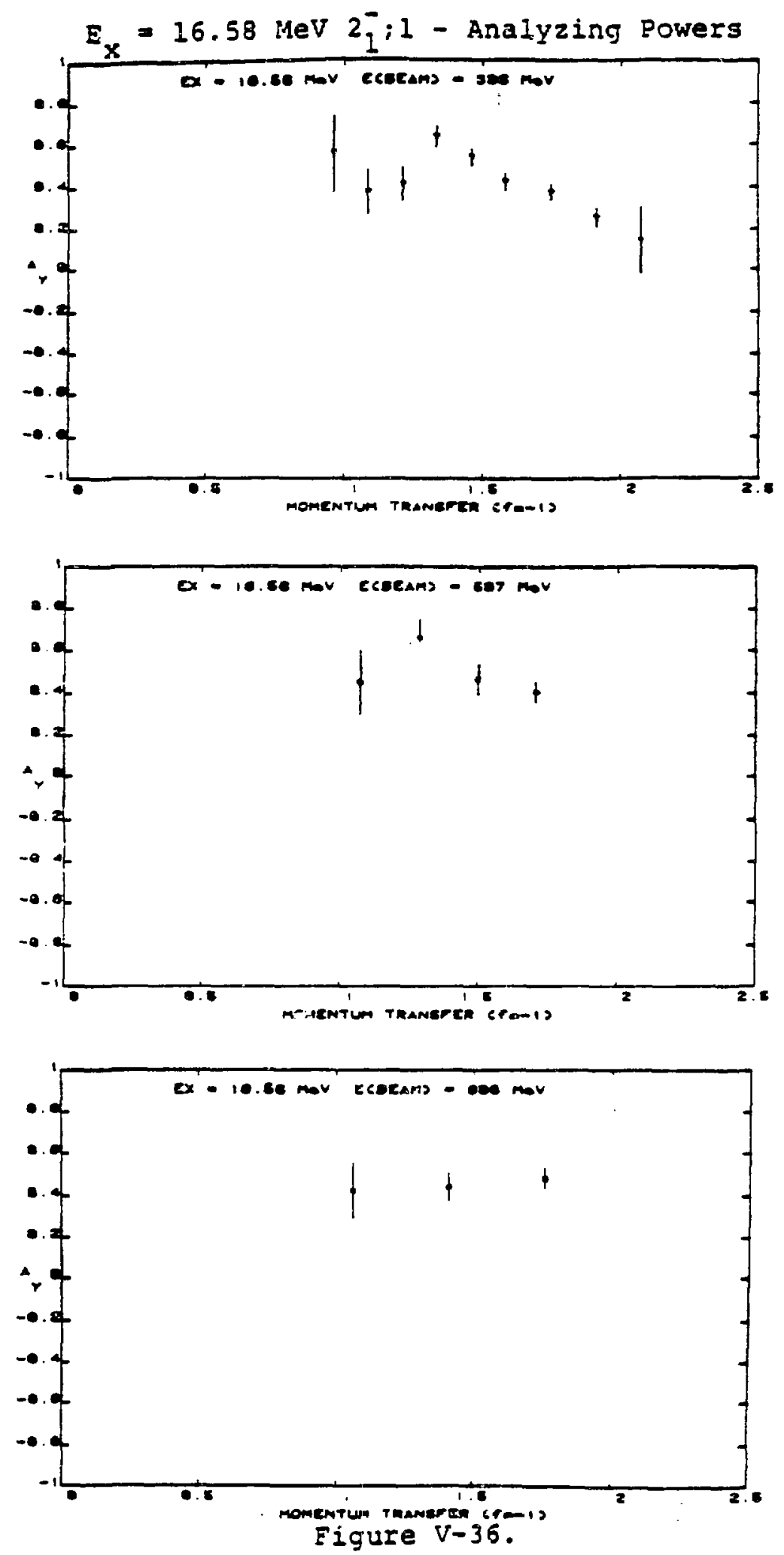
$E_{X}=16.58 \mathrm{MeV} 22_{1}^{-}: 1-$ Comparative Observables
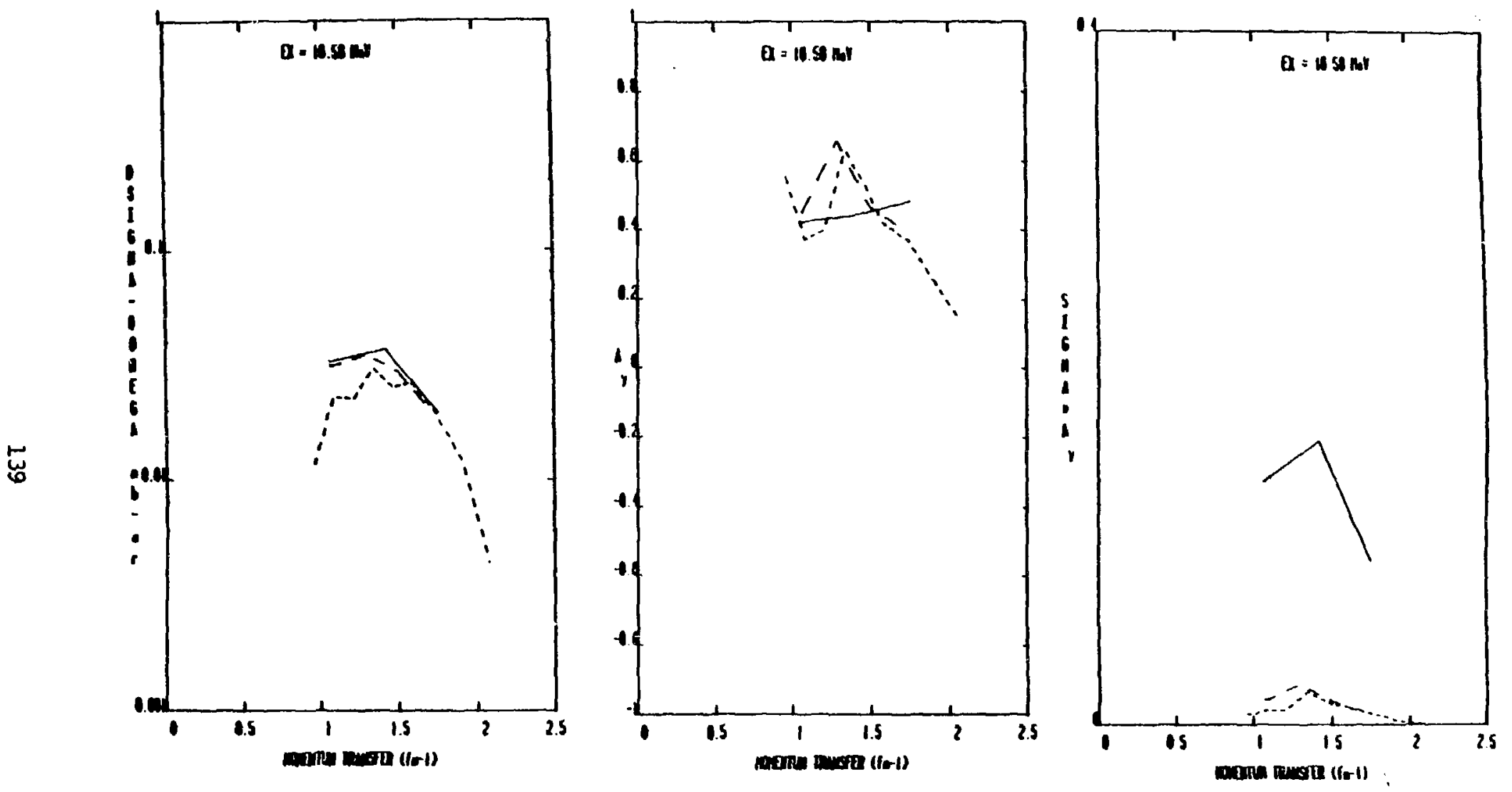

Figure V-37. 
first appear. No significant deductions can be made from these data.

5) States of indefinite quantum numbers for $E_{x}$,
$18.0 \mathrm{MeV}$ We present here data for five states in the excitation energy region $18.0 \mathrm{MeV} \leq \mathrm{E}_{X} \leq 21.6 \mathrm{MeV}$. The exact spin, parity and isospin assignments for these states are open to question, and the discussion of the DWIA analysis in the next chapter will address such assignments directiy. In this section we endeavour to discuss the systematics of these states and the similarity or dissimilarity of these systematics with those discussed previousiy for more weil known states.

(a) The state at $E_{x}=18.30 \mathrm{MeV}$

Date for this state are presented in Figures $V-38$ to $v-40$. One of the more striking features of the data is the significant strength observed for $q \leq 0.5 \mathrm{fm}^{-1}$. The shape of the differential cross section is seen to resembie that of the $T=1+$ transition, aithough the behaviour for small values of $q$ is different. These data show a weli-derined rise to a maximum value for $q-0.8 \mathrm{fm}^{-1}$, while the $\varepsilon_{x}=$ 12.7I Mev data are constant for smail values of $q$. The data for $E_{p}=398 \mathrm{MeV}$ show definite minimum in the $q=2.0 \mathrm{fm}^{-1}$ region which is unfortunateiy not observabie at the higher 

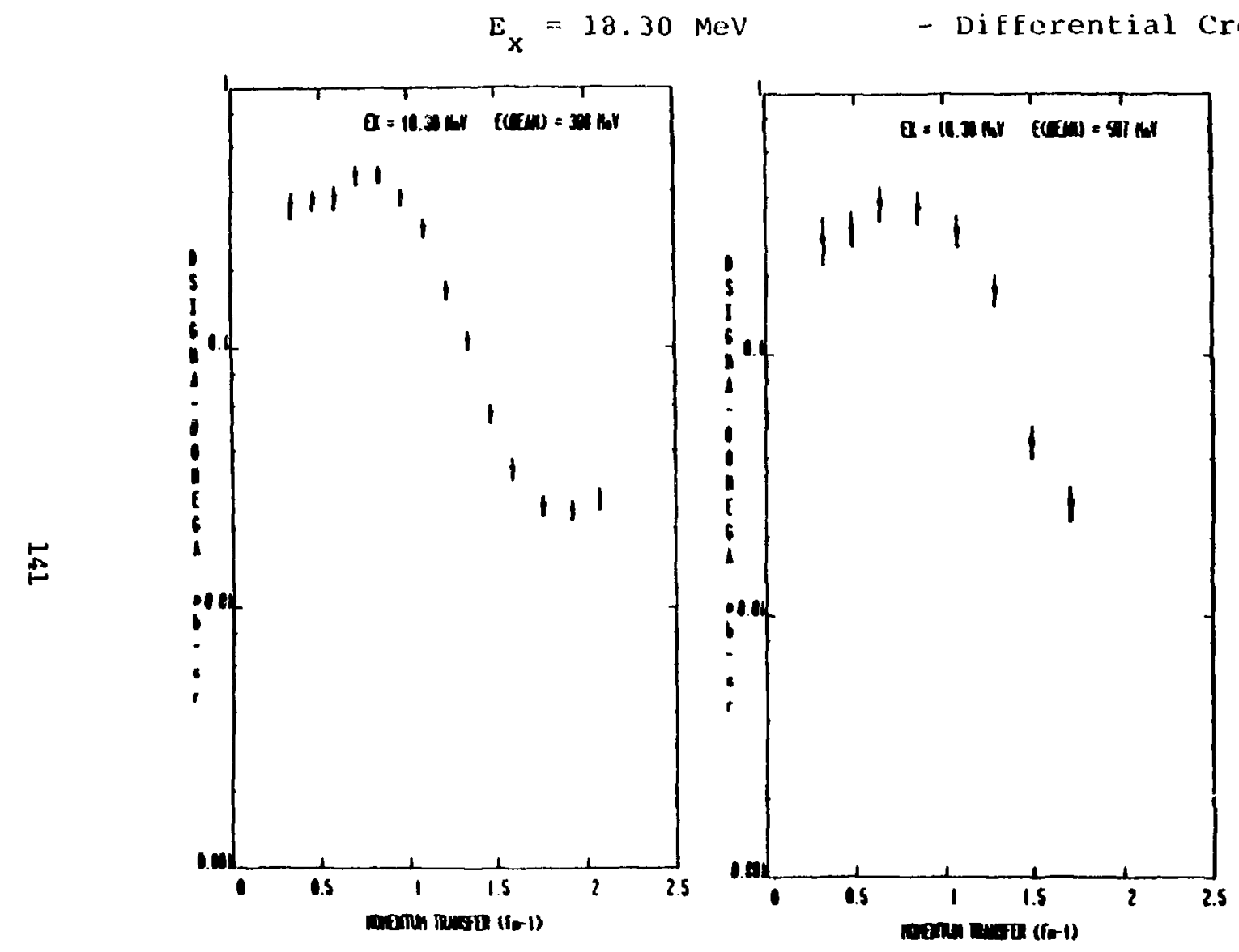

Cross Sections

igure V-38.

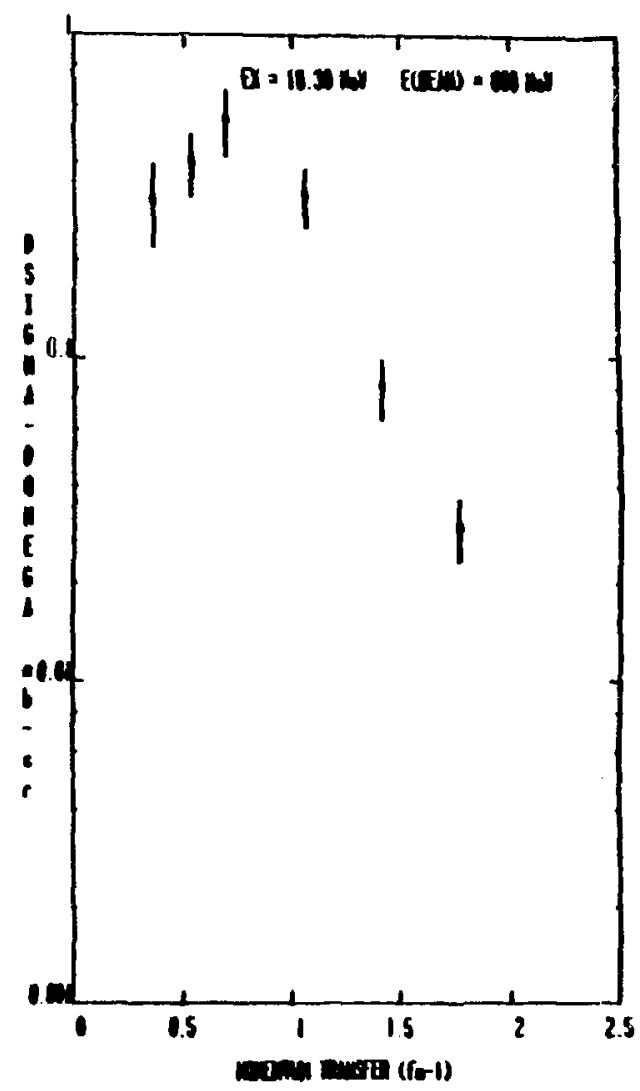



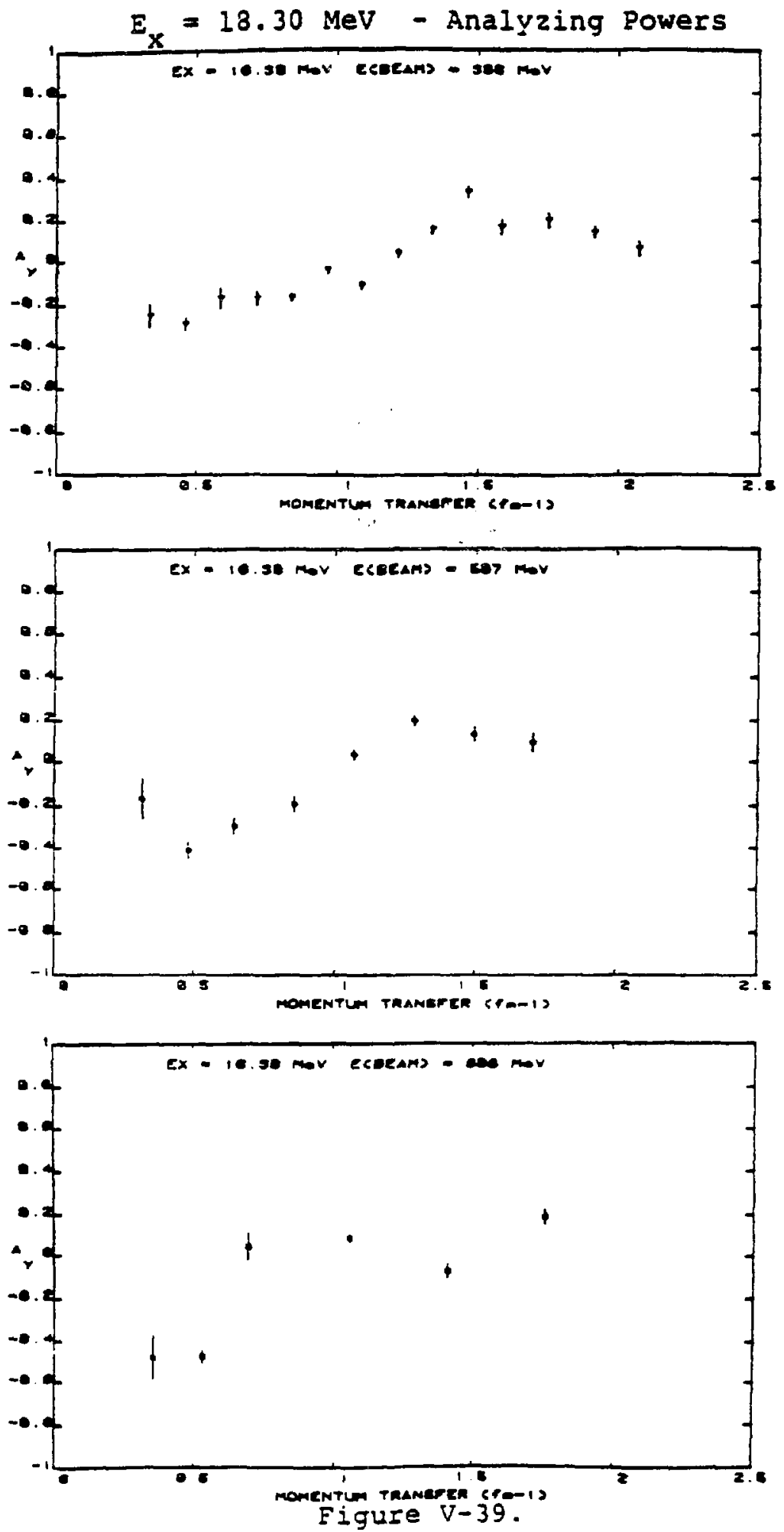
energies because the cata do not extend this far. Examination of Elgure $V-40$ shows evidence for the unnatural parlty character of this state. The comparative piot of the differential cross sections for the different beam energies shown if Flgure $V-40$ exhibits the same independence of incident projectile energy as is seen for isoscalar or isovector unnatural parlty states and and isovector states of natural parity of lower excitation energy.

The anaiyzing power data of figure v-39 provide some ev1dence for assignment of an lsoscaiar nature to this transition. The andyzing power for ail incident proton energies is cieariy negative for smaij values of q, Iising to positive vaiues as $q$ increases. The crossover point from negative to positive values decreases as $E_{p}$ increases. These observations are entirely consistent with the systematic behaviour of $A_{y}$ for the isoscaiar $1^{+}$state at $E_{X}=12.71$ Mev, thereby providing some motivation for assigning isoscaiar properties to this transition.

The product of the differentiai cross section and the analyzing power as shown in Flgure $V-40$ show features remarkably simflar to those observed for the 1 soscaiar $1+$ transition. Strongly negative vaiues are seen for smali values of $q$, rising to values consistent with zero as $q$ increases.

The systematics of the observabies measured for this 
-ransition thus suggest that it is an lsoscaiar transition of etther natural or unnatural perity. The data, though, provide no basis for a spin or parity assignment. Possible assignment of spin and parity, together with more restrictive tests of the deductions made here require the resuits of DWIA calculations which are presented in the next chapter.

(b) The state at $E_{x}=19.28 \mathrm{MeV}$

The data for this state are presented in Figures V41 to $v-43$. This state is not populated at ali for values of $q$ less than $1.2 \mathrm{fm}^{-1}$. For q iarger than this value, it is seen for all incident beam energles. Thls particular state is one of the broadest particle-hole states seen in the spectra, having a width of $580 \pm 50 \mathrm{kev}$. The fact that no strength is observed for smail values of g suggests that the spin of this state must be iarge, possibiy 3 or 4 . The measured differential cross sections are of the order of 0.1 $\mathrm{mb} / \mathrm{sr}$ or iess, and as can be seen from Figure $V-41$ it is not possible to make definite observations concerning the structure of the cross section.

The analyzing powers are generaliy negative for values of the momentum transfer less than $1.8 \mathrm{fm}^{-1}$, rising to values near zero for larger $q$. The product of the differential cross section and $A_{y}$ does not yleld any significant information. 


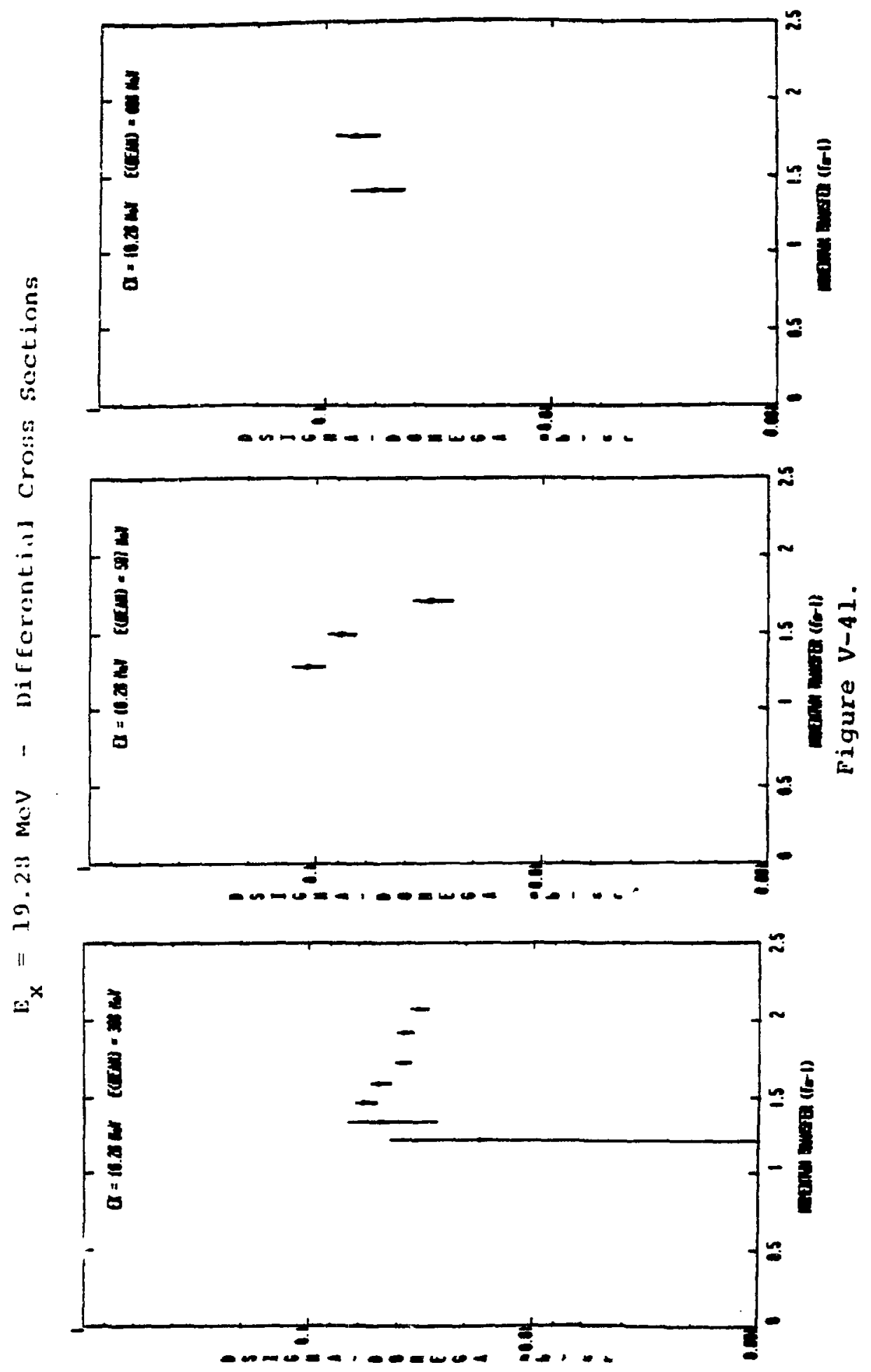



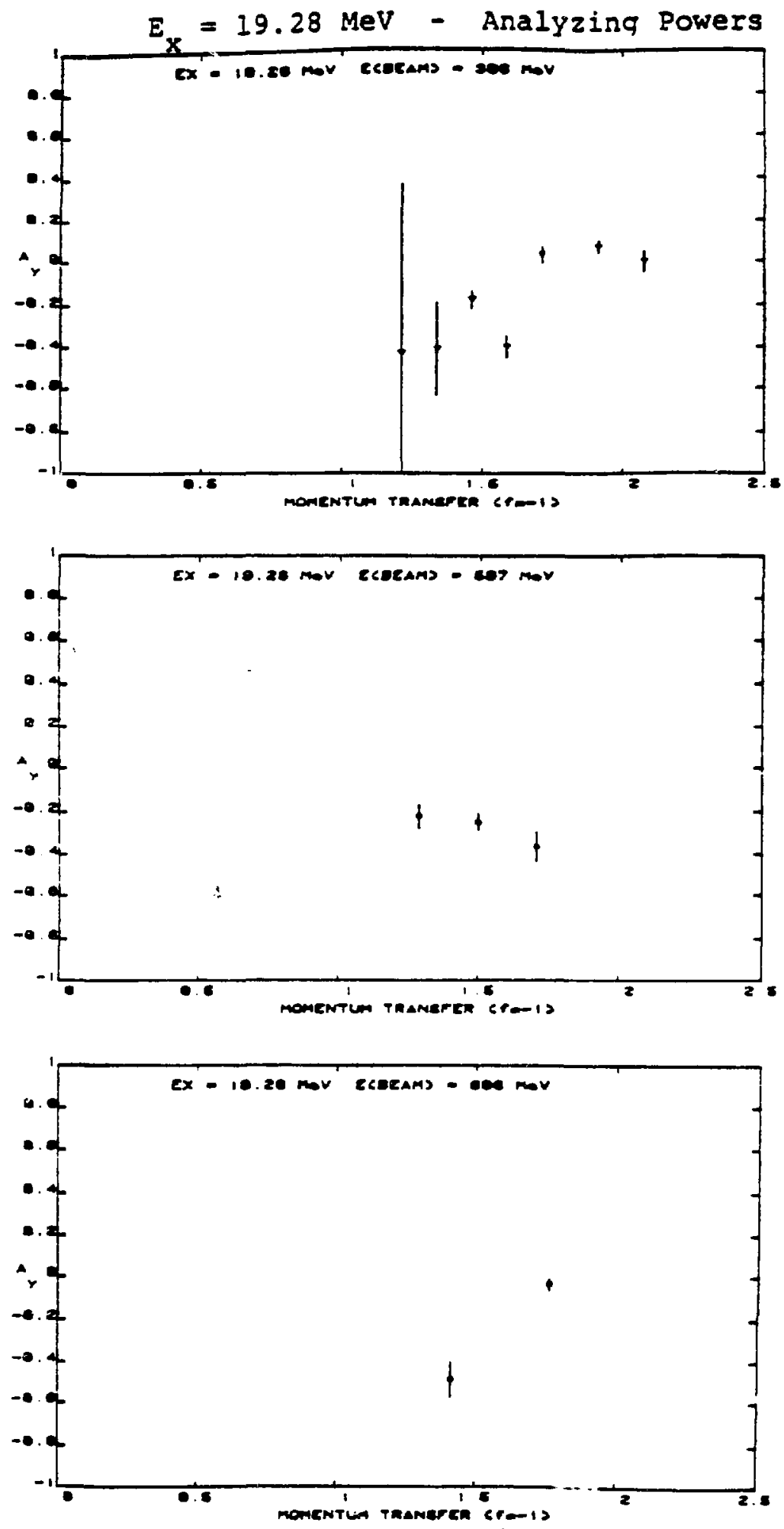

Figure V-42. 
$\mathrm{F}_{\mathrm{x}}=19.28 \mathrm{McV}-$ Comparative Observables
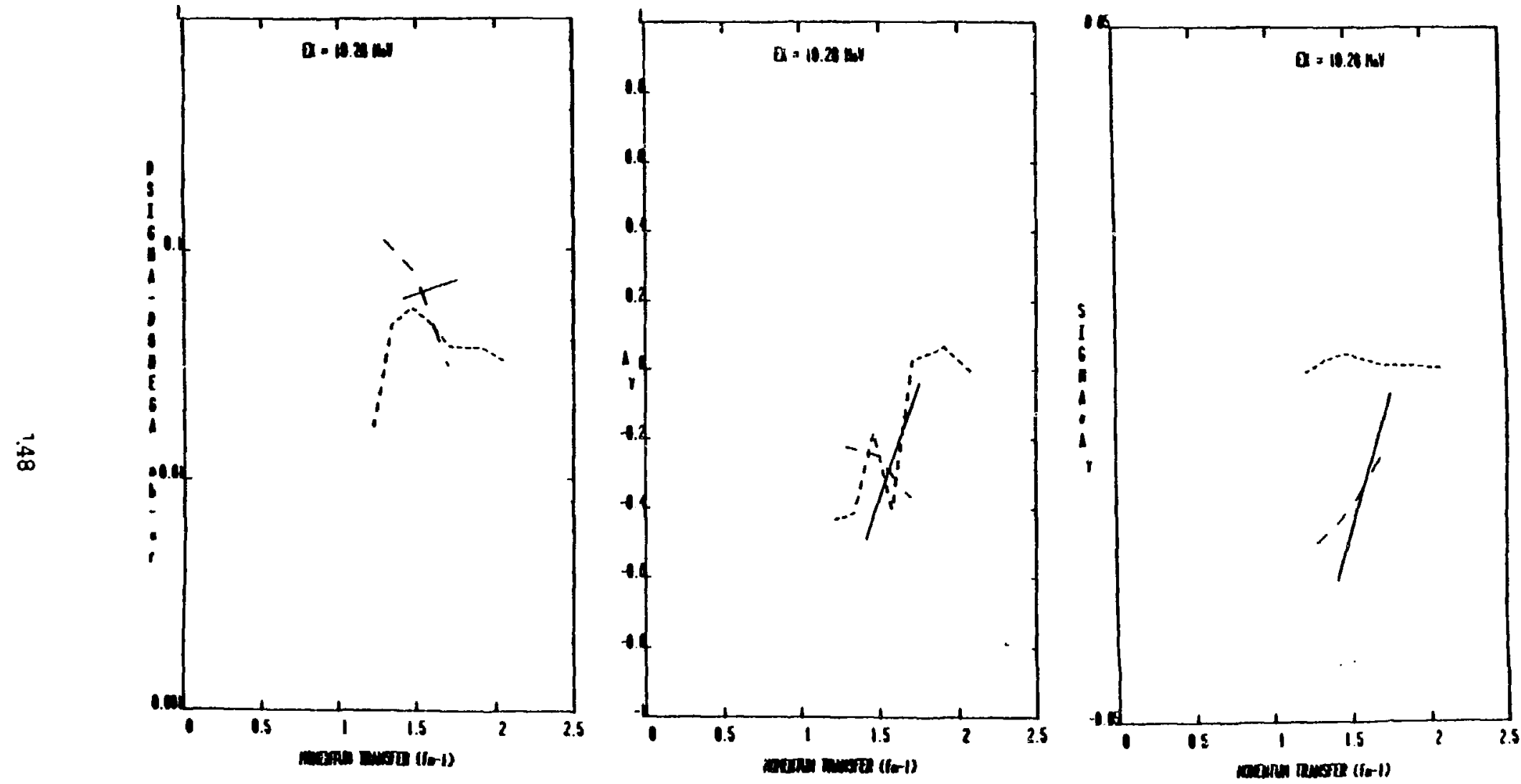

Figure V-43. 
Other than the natural parity $3^{-}$and $4^{+}$isoscalar states discussed previousiy, no other 'high' spin states have been seen in the spectra up to this excitation energy. The negative character of the anaiyzing power differs Erom that observed for the isoscalar natural parity states suggesting that this transition may be of unnatural parity, but no deductions may be made concerning the isospin nature of this states.

(c) The state at $E_{x}=19.40 \mathrm{MeV}$

The data for this transition are presented in Figures $V-44$ to $V-46$. The differential cross section data presented in figure $V-44$ show strong peaking for small values of $q$. Furthermore, the strength associated with this transition at the smailest vaiues of $q$ is of the order of 1 mb/sr, oniy a factor of about 2.5 smajier than that observed for the $1^{+}$il state in the same region of $q$. The data fail off rapidiy as $q$ Increases, and for vaiues of g greater than about $1.2 \mathrm{fm}^{-1}$, no strength couid be extracted. Examination of Figure V-46 shows that the measured cross section is independent of incident particle energy, whlch has been shown to be representative of isoscalar and isovector transitions of unnatural parity, or isovector transitions of natural parity. The strong forward peaking of the cross section is suggestive of a state of low spin (2 or less) of unnatural parity, but previous systematics cannot preclude the pres- 
$E_{x}=19.40 \mathrm{MeV}-$ Differential Cross Sections
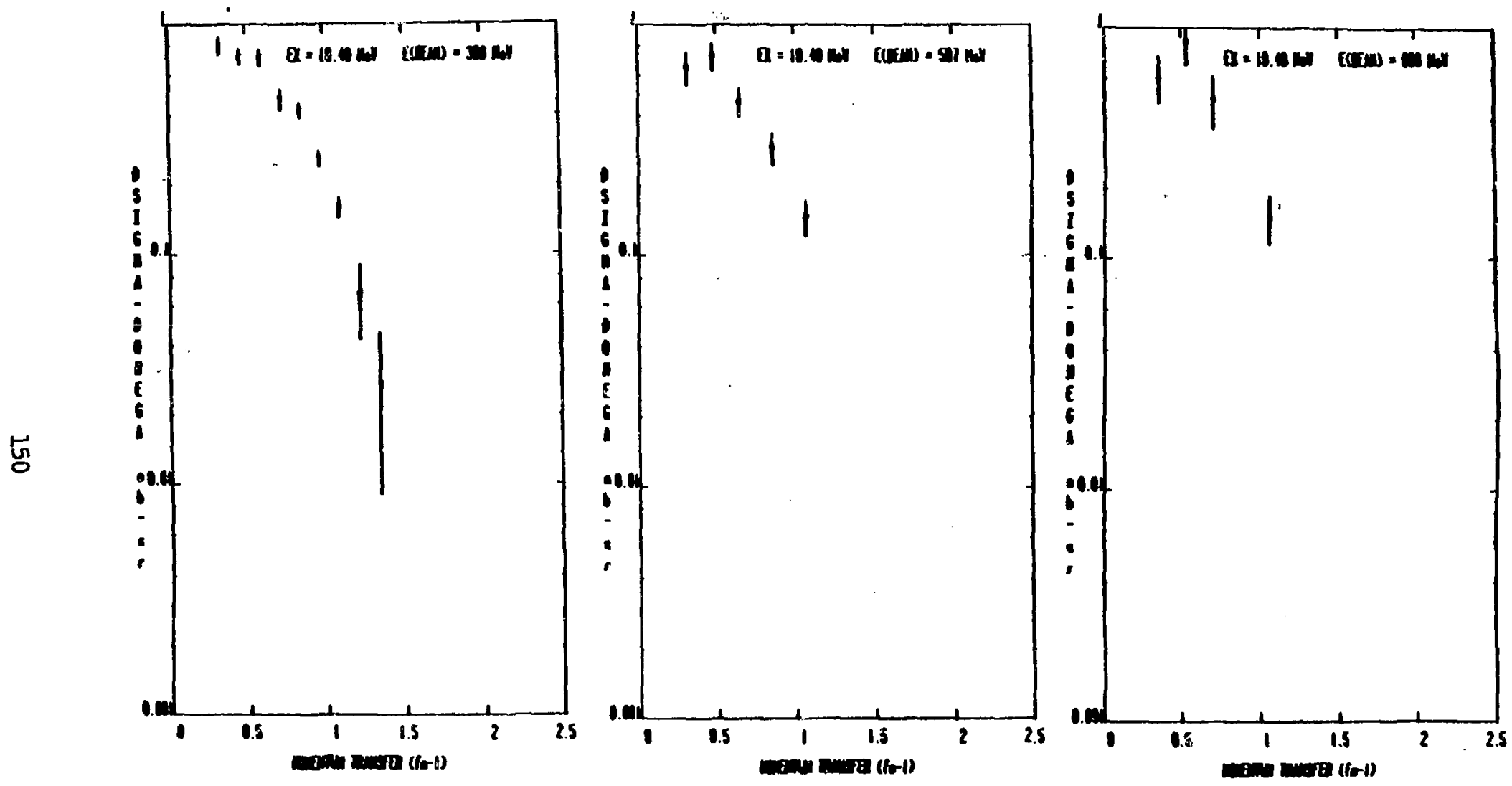

Figure V-44. 

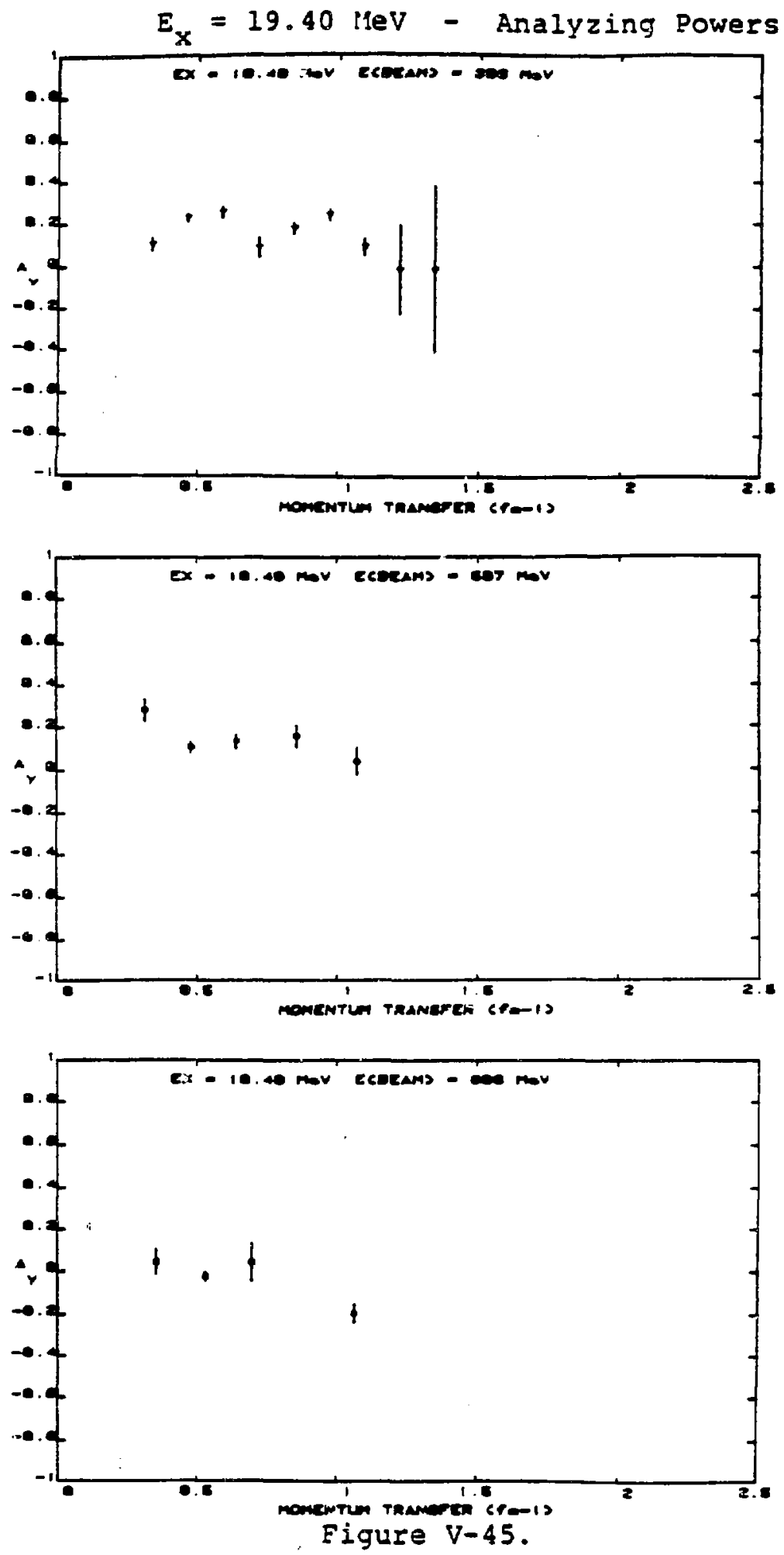
$E_{x}=19.40 \mathrm{MeV}$ - Comparative Observables
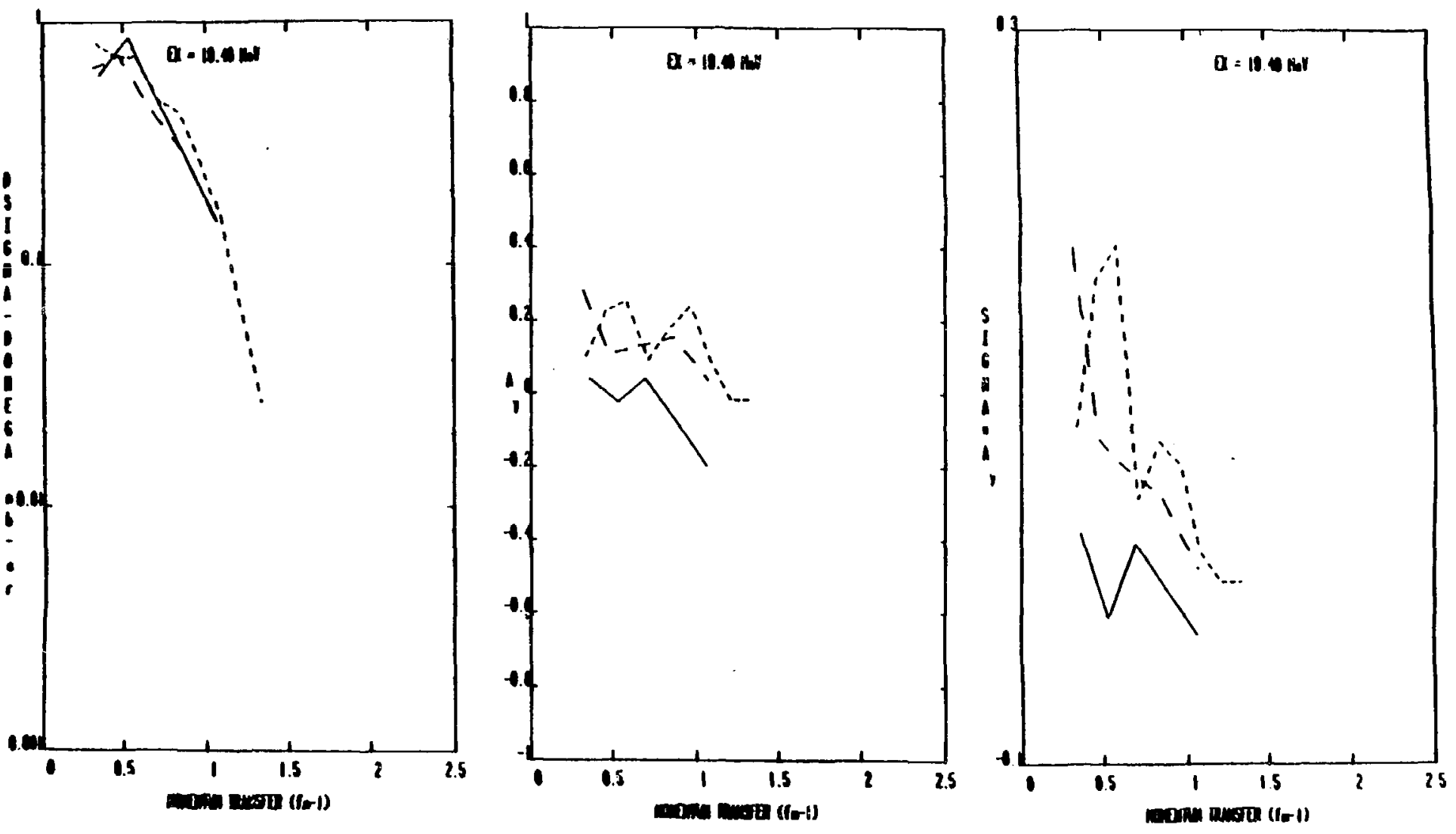

Figure $V-46$. 
ence of isovector components of natural parity.

The analyzing powers, presented in Figure v-45, provide some useful information when compared with previously discussed data. Fo. smail values of $q, A_{y}$ is generally consistent with zero, showling a trend toward negative values for $q \geq 0.8 \mathrm{fm}^{-1}$. Comparison with the analyzing power data for the $1^{+}$il transition show remarkabie similarities, suggesting that this transition has a dominant unnatural parity I sovector component.

The data for the product of the observables is in general positive for smali values of $q$, decreasing to zero as $q$ increases, reflecting the rapld decrease in magnitude of the differential cross section. The behaviour of this product is somewhat similar to that for the $1^{+}$il state. aithough their are considerabiy fewer data points for this transition.

It is therefore possibie to conciude on the basis of the systematics of the data that this transition shouid be dominated by a low spin isovector unnatural parity component, although the presence of 3 low spin isovector natural parity component cannot be ruled out.

(d) The state at $E_{x}=19.65 \mathrm{MeV}$

The data for this state are presented in Figures $V$ 47 to $v-49$. The differential cross section data shown in 
$E_{x}=19.65 \mathrm{MeV}-$ Differential Cross Sections
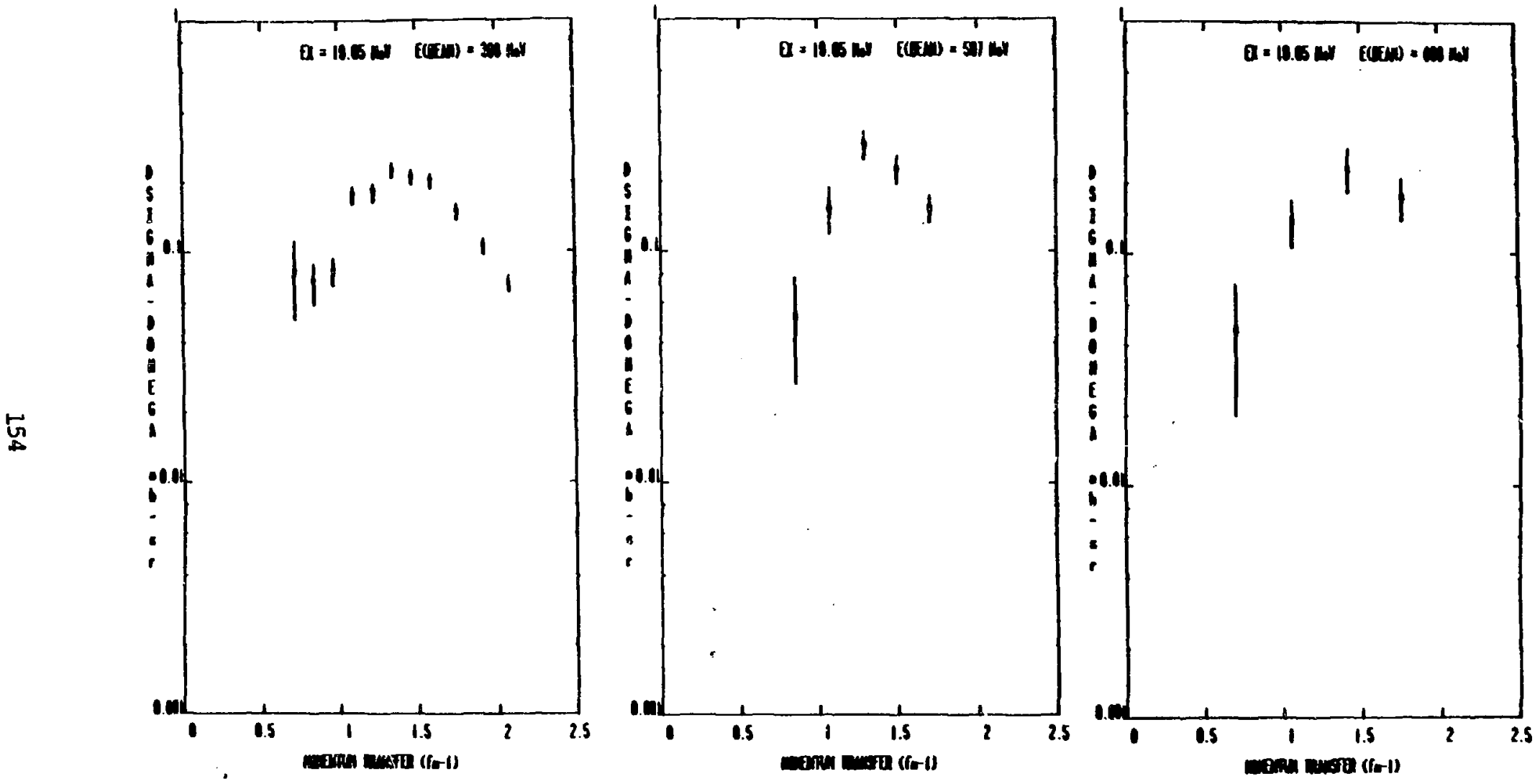

Figure $v-47$. 

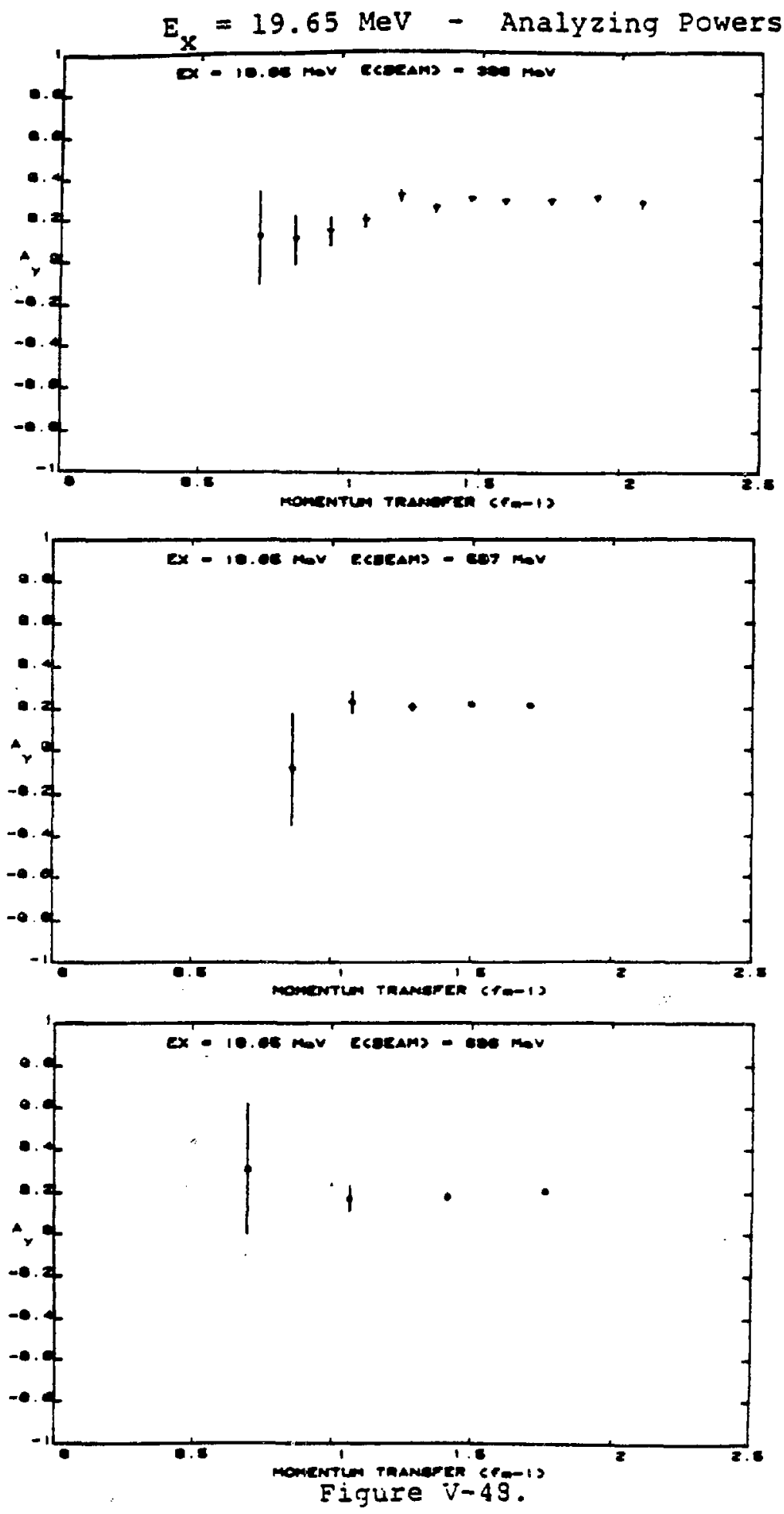
$E_{x}=19.65 \mathrm{MeV}-$ Comparative Observables
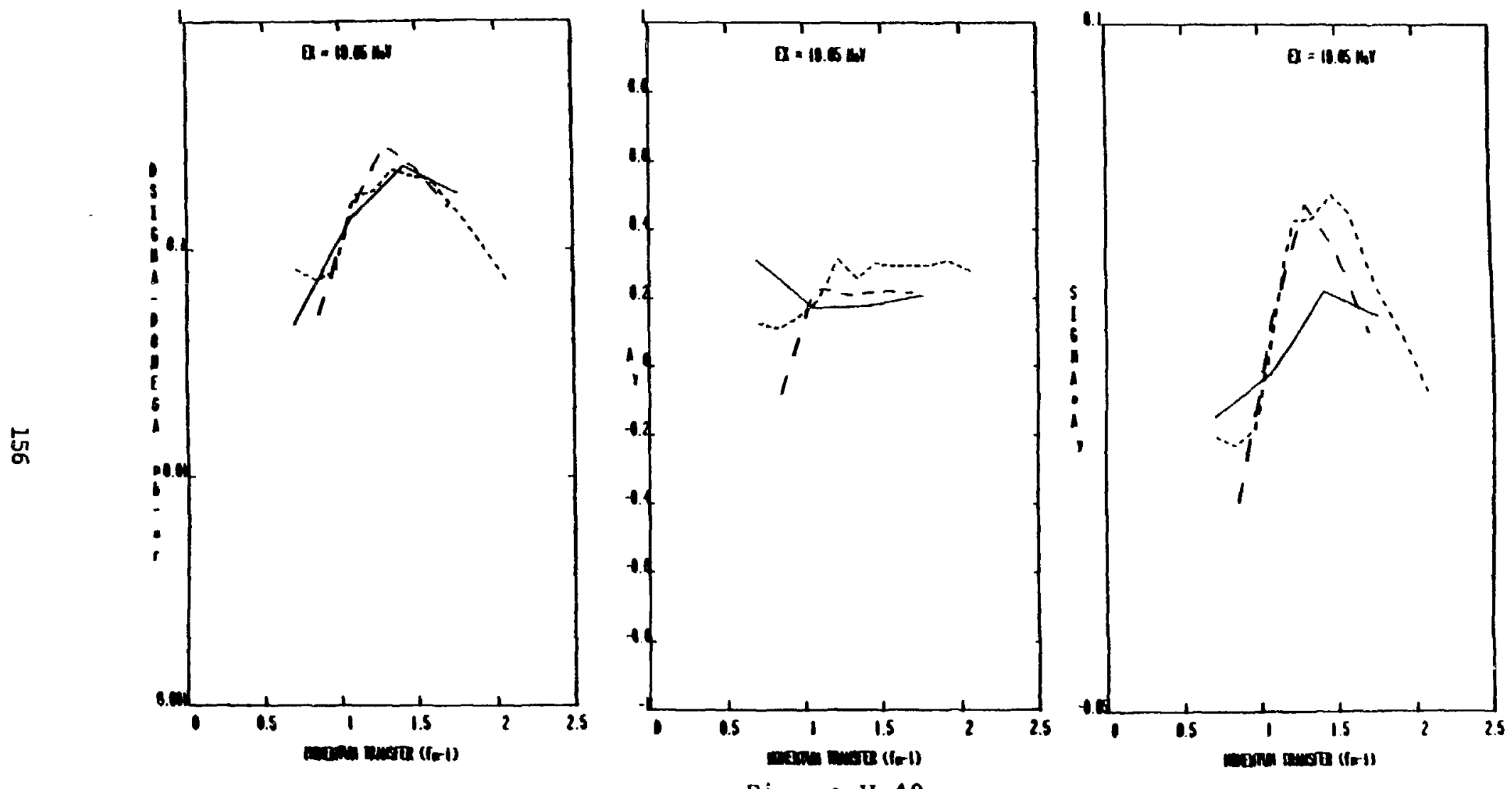

Figure V-49. 
Figure $V-47$ show a distinct maximum for $q-1.4 \mathrm{fm}^{-1}$. The shape of the data is consistent with a spin assignment of 3 or 4. As has become common for states in this exciation energy region, the differentiai cross section show aimost no dependence on the incident proton energy, thus suggesting a state of unnatural parity, or an lsovector state of natural parity. The maximum value attalned by the differential cross section is of the order of $0.25 \mathrm{mb} / \mathrm{sr}$.

The analyzing power does not exhiblt much structure, being near zero for $0.8 \mathrm{fm}^{-1} \leq q \leq 1.1 \mathrm{fm}^{-1}$. For $q>1.1$ $\mathrm{fm}^{-1} \mathrm{~A}_{\mathrm{y}}$ is consistentiy positive, and about 0.2 . The absence of significant structure in the anaiyzing power data does not aijow any further conclustons concerning the 1 sospin nature of the transition.

The product of the differentiai cross section and $A$ shows a reasonabie constancy as a function of $E_{p}$, and its structure is dominated by the structure of the cross section as $A_{y}$ is almost constant. Further information on the quantum number assignments for this state requires the resuits of the DWIA computations presented in the next chapter.

(e) The state at $\varepsilon_{x}=20.60 \mathrm{MeV}$

The data for this state are presented in Figures V50 to $v-52$. The data for the differential cross section show a maximum for $q-0.9 \mathrm{fm}^{-1}$. The shape of the data is 


\section{$\mathrm{E}_{\mathrm{x}}=20.60 \mathrm{MeV}-$ Differential Cross Sections}
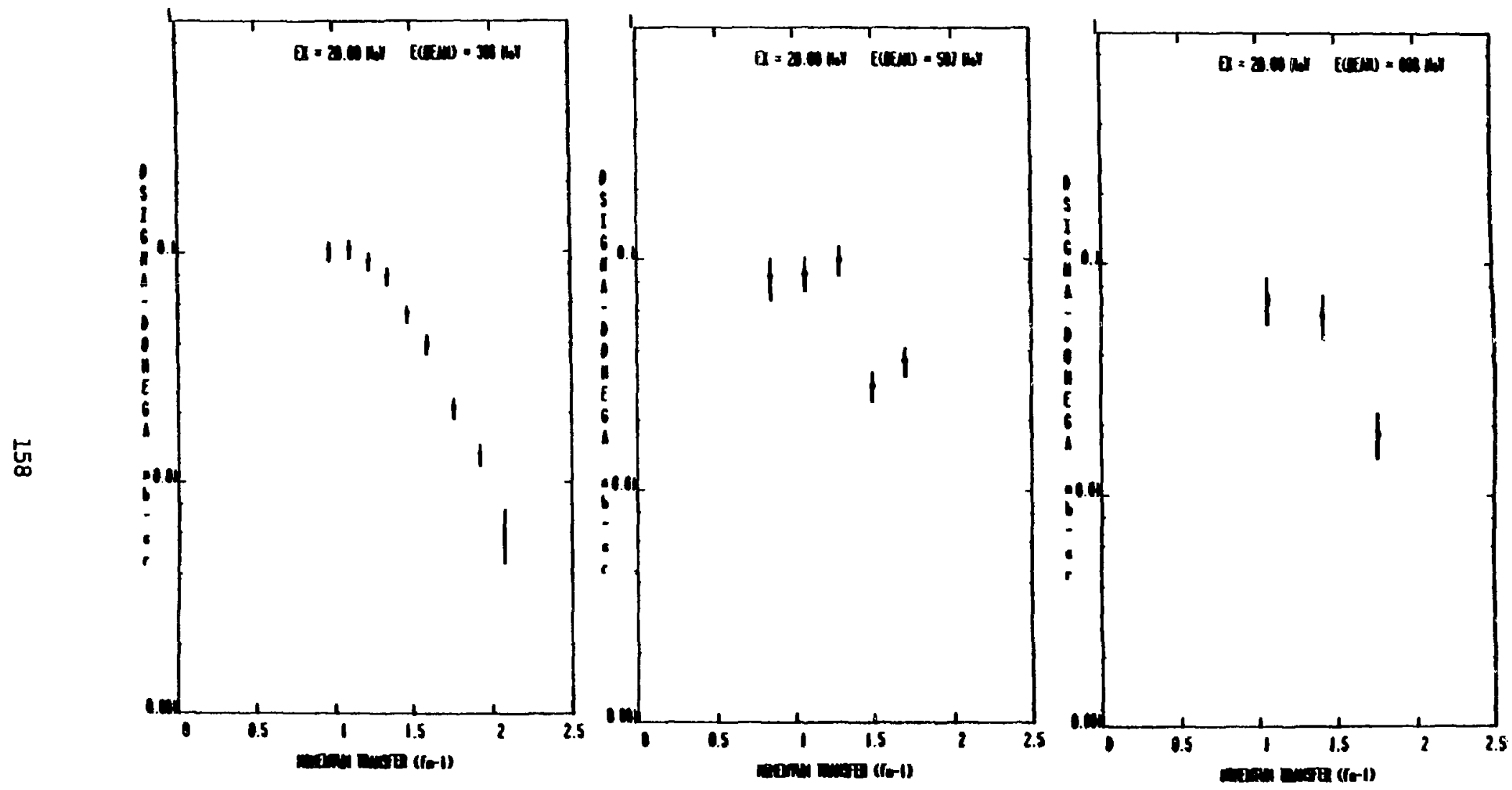

Figure V-50. 

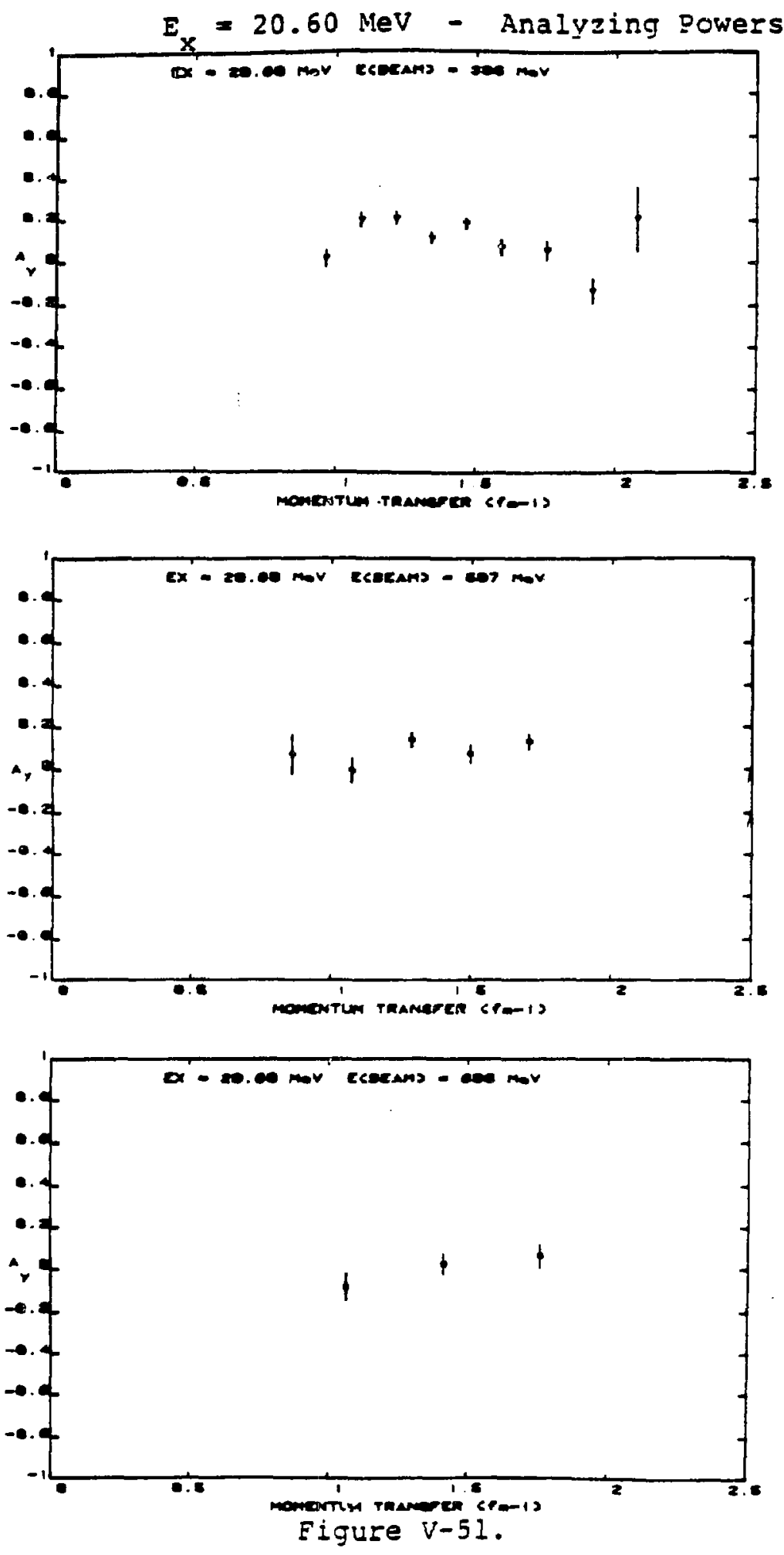


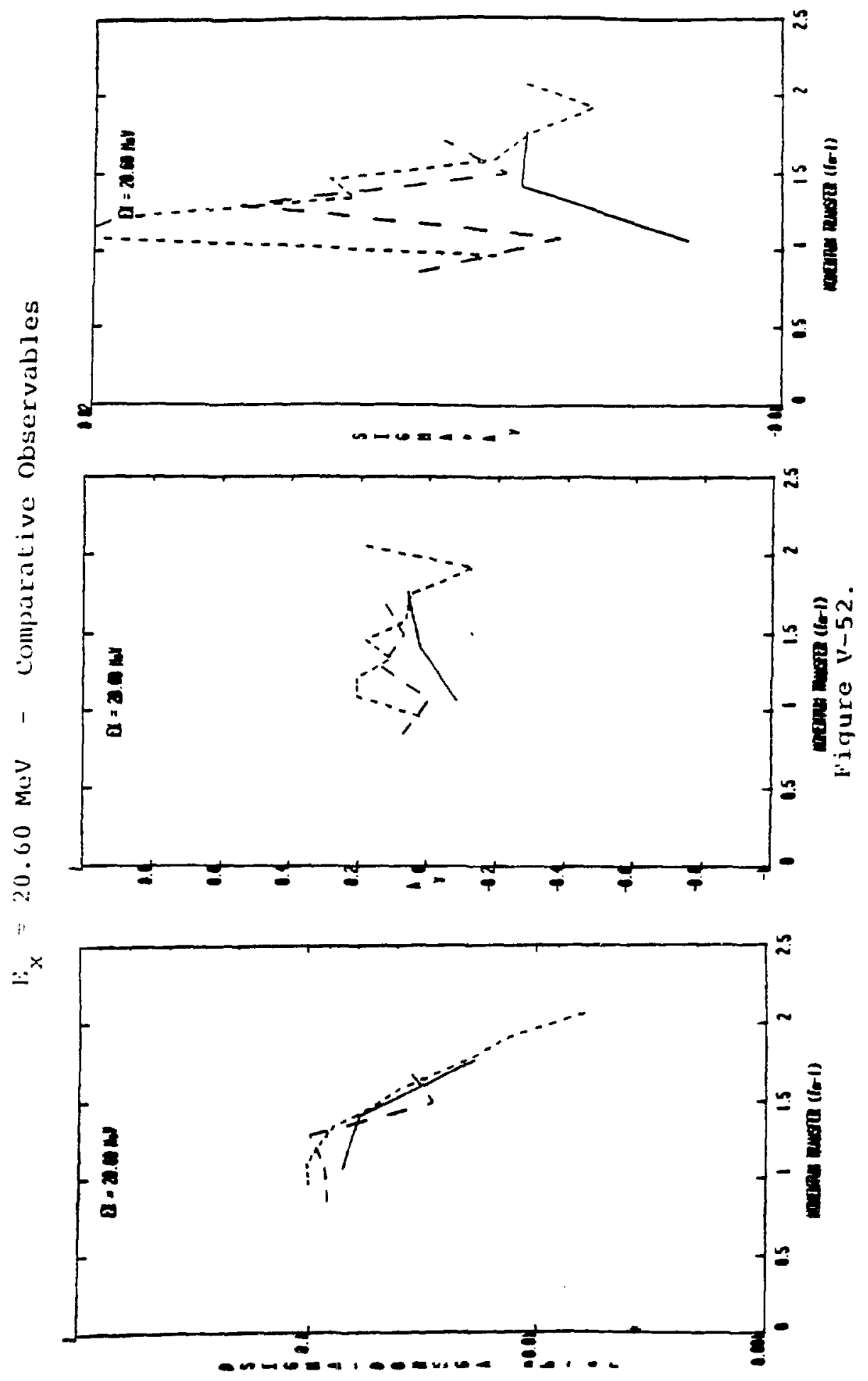


consistent with a spin of 2 or 3 , as is evidenced by comparison with the data for 1 soscalar states of natural parity. Examination of Figure V-52 shows, however, that the magnitude of the differential cross section is independent of incident beam energy, and that consequently this transition is of unnaturai partty or possibly an isovector transition of natural parity.

The analyzing power data are, in general, smali in magnitude and positive over the range of $q$ for which data were taken. Such behaviour is consistent with that observed Eor the state at $\Sigma_{x}=19.65 \mathrm{MeV}$, so it might be expected that the isospin properties of this transition could be the same as that of the $19.65 \mathrm{MeV}$ state. This deduction is of use when comparisons with DWIA calculations are made.

The structure of the product of the cross section and $A_{Y}$ provides little additional information, other than to exhibit a dependence on $q$ similar to that for the $19.65 \mathrm{MeV}$ state. The positive nature of the anaiyzing power would seem to suggest a transition of isovector nature, although this cannot be taken as strong evidence for such an assignment. 
VI. THEORETICAL INTERPRETATIONS OE THE DATA

We present here the results of theoretical caicuiations performed to aid in the interpretation of the data presented in the preceding chapter. Optical model computations for the elastic scattering data are presented first, together with a sumary of the potentlais resulting from these computations. Resuits of Distorted Wave Impuise Approxtmation caicuiations for a variety of transitions are then presented, and theit validity and consequences discussed.

A) Opticai Model Calculations - Elastle Scattering $\therefore$ 'A prerequisite for DWIA caiculations is knowledge of an average potentiai capable of describing the incident and exlt channei elastic scattering. Such optical model parameters were not readily available for the incident beam energies used in this work. The optical modei search program CUPID46) was used to determine an optimal set of potential parameters which best reproduced the measured eiastic scattering differential cross section and analyzing power at each incident beam energy.

The code accepts, as Input, data for both the differential cross section and the analyzing power, together with initial estimates for the strengths, radil, and difEusenesses of real and imaginary centrai and spin-orbit potentials. The shape of the potential weil used in the com- 
putations is the standard Woods-Saxon form, and reiativistic kinematics was used throughout.

The data for $E_{p}=398 \mathrm{MeV}$ were fitted first. A se: of previously determined parameters ${ }^{47}$ was used as an initial estimate. Both cross sections and analyzing powers were fitted simultaneously, and the full set of twelve adjustable parameters (four potentials, four radil and four difEusenesses) ware varied to minimize chi-squared. An adjustable normalization factor was held fixed at 1.0 throughoue. The best fits to the data obtained in this way are presented in Figures VI-I and VI-2.

The potentlal parameters derived in the fitting procedure outlined above are presented in Tabie VI-I, together with the resuiting normalized chi-squared vaiues indicating the overall quaitiy of the fit to the dara for the tabuiated parameters. The overall chi-squared parameter is dominatea by contributions from the analyzing power.

We note that the guaifty of the fit to the differential cross section data is in general good. Some doviations are seen for the smallest scattering angies measured, as well as in the reglon of the minimum and the second maximum. For the angular reglon $8^{\circ}$ to $16^{\circ} \mathrm{CM}$, the fit is seen to slightly underestimate the magnitude of the cross section data. The fit to the analyzing power is not as good as that to the cross section. While the minimum in $A_{y}$ is weil 


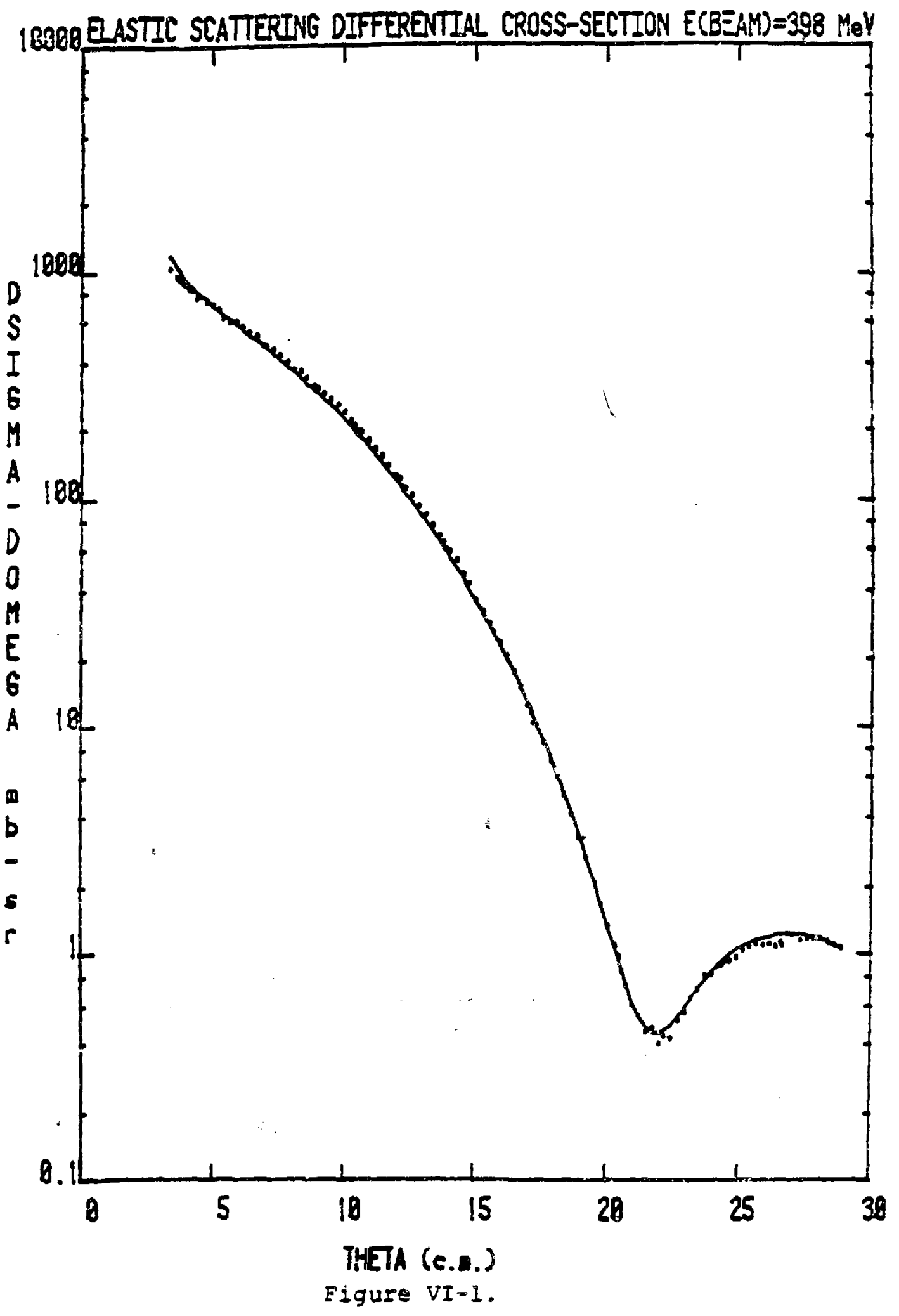




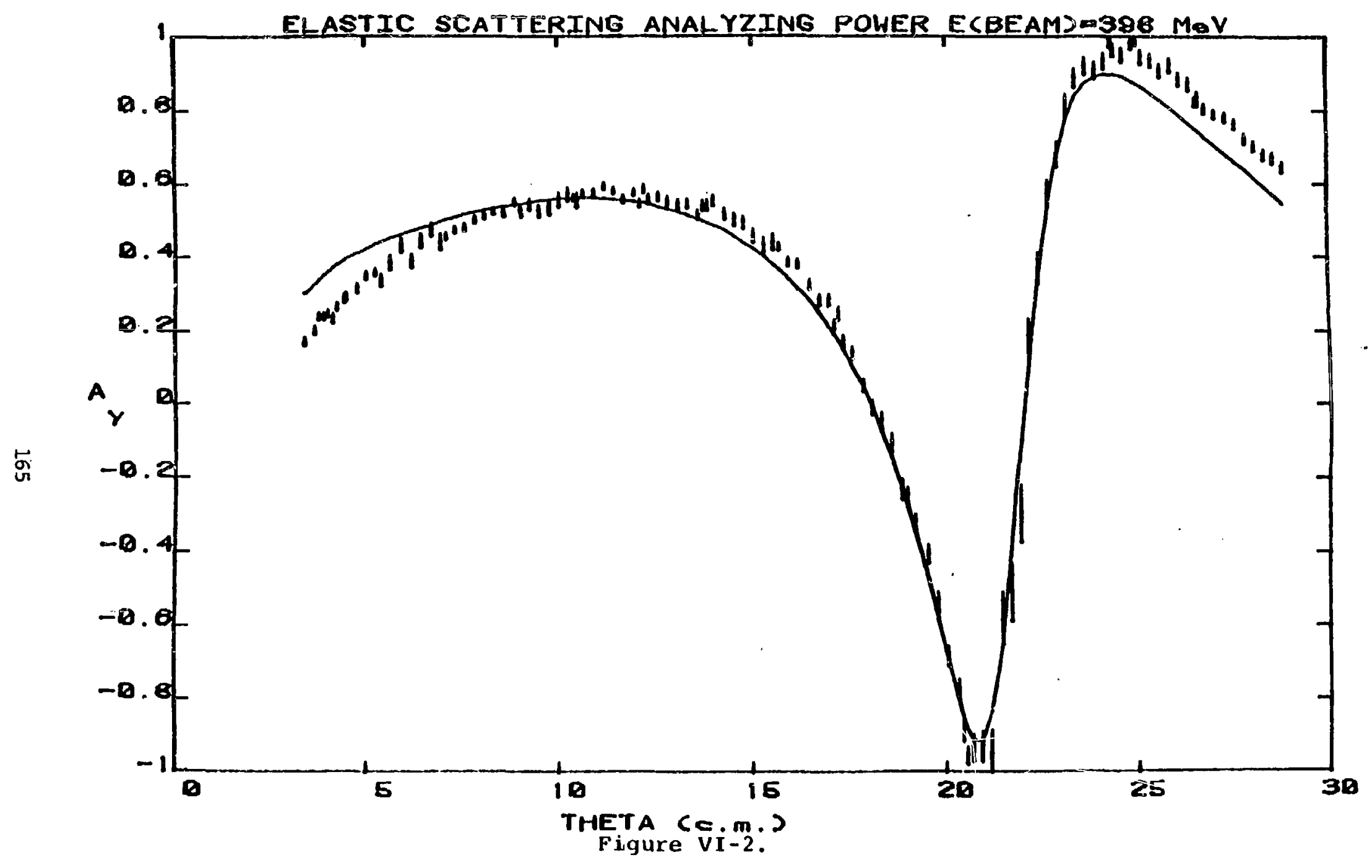


Optical Model Potential Paramaters

\begin{tabular}{|c|c|c|c|}
\hline & $400 \mathrm{MeV}$ & $600 \mathrm{MeV}$ & $700 \mathrm{MeV}$ \\
\hline $\begin{array}{l}V \\
W \\
v_{15} \\
W_{1 s} \\
w_{5}\end{array}$ & $\begin{array}{l}-2.62 \mathrm{MeV} \\
-25.9 \\
-7.88 \\
-11.3 \\
-\end{array}$ & $\begin{array}{l}1.10 \mathrm{MeV} \\
-78.8 \\
-4.89 \\
-14.3 \\
-2.37\end{array}$ & $\begin{array}{l}1.84 \mathrm{MeV} \\
-93.5 \\
-4.67 \\
-18.4 \\
-2.58\end{array}$ \\
\hline $\begin{array}{l}r_{V} \\
r_{W} \\
r_{V 1 s} \\
r_{W 1 s} \\
r_{s}\end{array}$ & $\begin{array}{l}1.25 \text { fm } \\
1.08 \\
0.995 \\
0.971 \\
-\end{array}$ & $\begin{array}{l}2.53 \mathrm{fm} \\
0.859 \\
1.01 \\
0.897 \\
1.94\end{array}$ & $\begin{array}{l}0.912 \mathrm{fm} \\
0.875 \\
0.908 \\
0.879 \\
1.59\end{array}$ \\
\hline $\begin{array}{l}a_{v} \\
a_{W} \\
a_{v 1 s} \\
a_{W 1 s} \\
a_{s}\end{array}$ & $\begin{array}{l}0.474 \mathrm{fm} \\
0.594 \\
0.496 \\
0.507 \\
-\end{array}$ & $\begin{array}{l}0.474 \text { fm } \\
0.594 \\
0.496 \\
0.507 \\
0.500\end{array}$ & $\begin{array}{l}0.474 \mathrm{fm} \\
0.594 \\
0.496 \\
0.507 \\
0.500\end{array}$ \\
\hline$x^{2}$ & 4.3 & 17.7 & 24.3 \\
\hline
\end{tabular}

Table VI-1. 
reproduced, the data for angles larger than $23^{\circ} \mathrm{CM}$ are underestimated. Furthermore the shape fo the calculated quantity differs significantly from the data from $3^{\circ}$ to $17^{\circ} \mathrm{cm}$. For the more forward angles in thle region the calculation overestimates the data, while underestimating the data for $10^{\circ}<\theta_{\mathrm{cm}}<17^{\circ}$.

The data for $E_{p}=597$ and $698 \mathrm{MeV}$ were fitted using the same procedure. For consistency, the diffuseness parameters determined in the fit to the data for $E_{p}=398 \mathrm{MeV}$ were held constant in all calculations for the two higher beam energies. Nomalization factors were also fixed at $1 . \emptyset$ to eliminate arbitrary renormalization of the fits. Reasonable fits to the data at these energies could not be obtained for searches on the standard central and spin-orbit potentials: The fits could not reproduce the depth of the minimum in the differential cross section; and also produced a much deeper oscillation in the analyzing power than is seen in the data.

It was noted that a set of optical parameters for proton scattering from ${ }^{12} \mathrm{C}$ at $E_{p}=8 \emptyset \emptyset \mathrm{MeV}^{47)}$ contained an imaginary surface absorptive term, the form of which is the first derivative of the Woods-Saxon shape. This term was included in further searches and was found to considerably inprove the quality of the fits obtained. The diffuseness Earameter for this term was held fixed at $9.5 \mathrm{fm}$. Fits to the data for $E_{p}=597$ and $698 \mathrm{MeV}$ so obtained are shown in 
Eigures VI -3 to VI -5

The fit to the cross, section data for $E_{p}=597 \mathrm{MeV}$ is excelient. The Elt to the cross section data for $E_{p}=698$ MeV is not as good, underestimating the data at forward angies and not reproducing the minimum as weil. The fits to the anajyzing power are not as good as that for $\varepsilon_{p}=398$ Mev. The mintmun is reasonabiy well reproduced in both cases. The sharp drop after the rise from the mintmum is not weil reproduced, and the differences in shape up to tine drop off to the minimum neted for $E_{p}=398 \mathrm{MeV}$ are seen to worsen as the beam energy increases. The data are overestimated at the most forward angles measured, and are badly underestimated for larger angles, convergence finaily being reached in the region of the dip to the minimum.

It should be pointed out that the simpie phenomenoiogical approach adopted in the analysis presented here is not necessarily the most fundamental analysis possibie, but it is very useful for DWBA cajcuiations. Giauber theory and the theory of kerman, McManus and Thaiez may provide a better understanding of the bahaviour of the phenomenologicai potentials derived in this analysis. The fitting procedure was difected primarily to optimizing the fit to the tza, and consequently zather ioose physlcal constralnts nz imposed on the parameters used in the search: The po$=y_{1}: a_{\text {i }}$ strengths were found to be reasonable, as were the at for the diffuseness of the woods-saxon reils, 


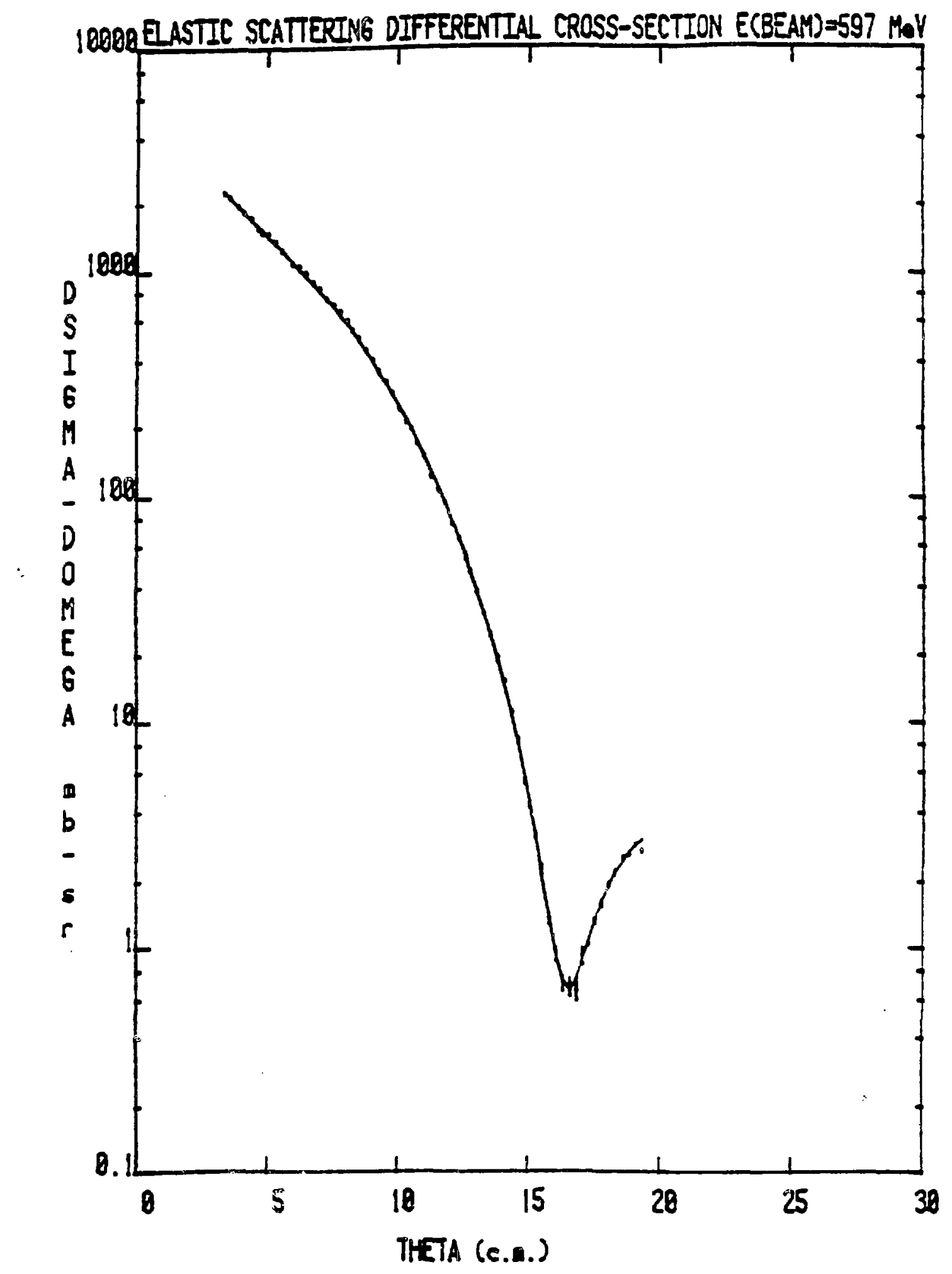

Figure VI-3. 


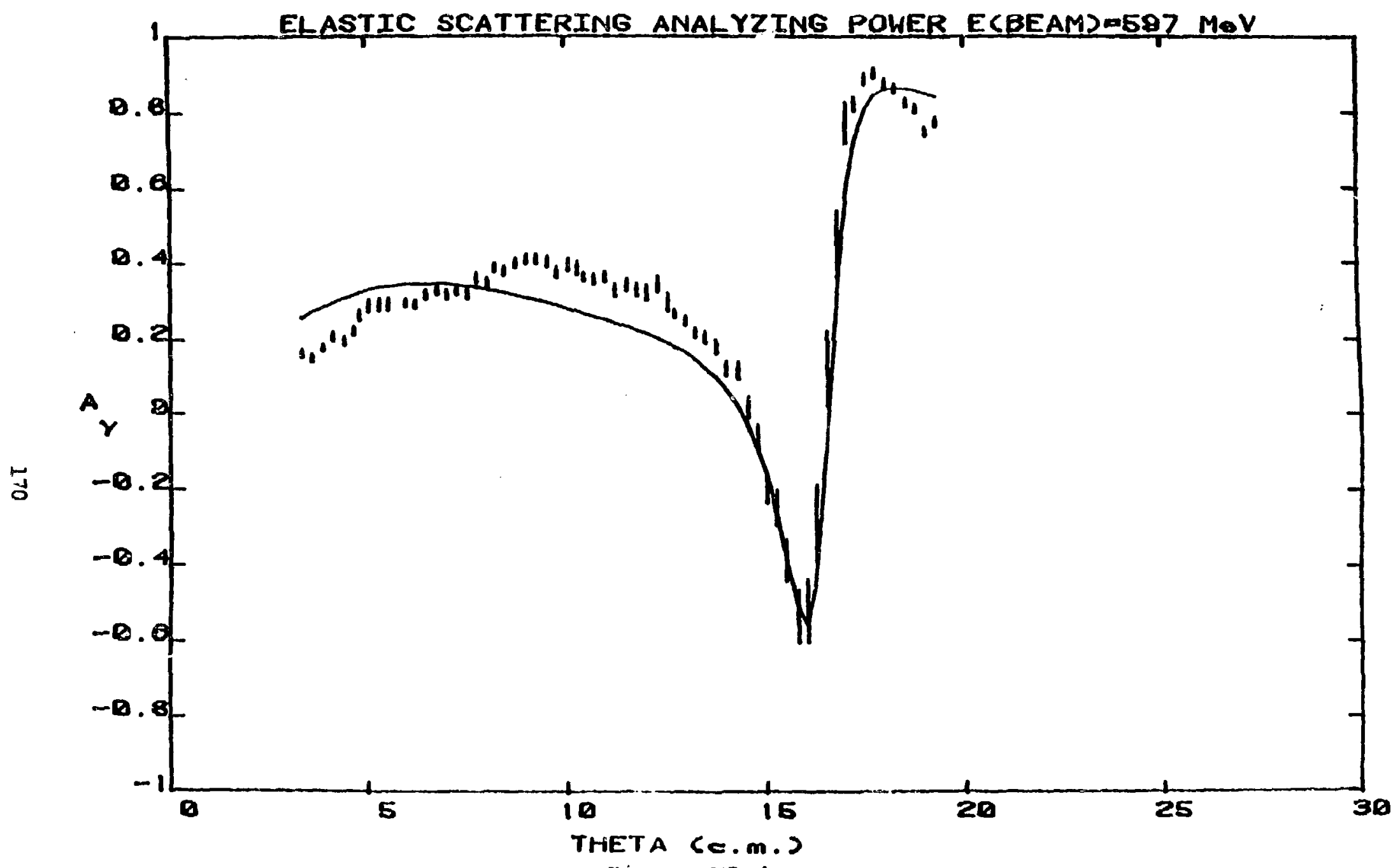

Pirure VI-4. 


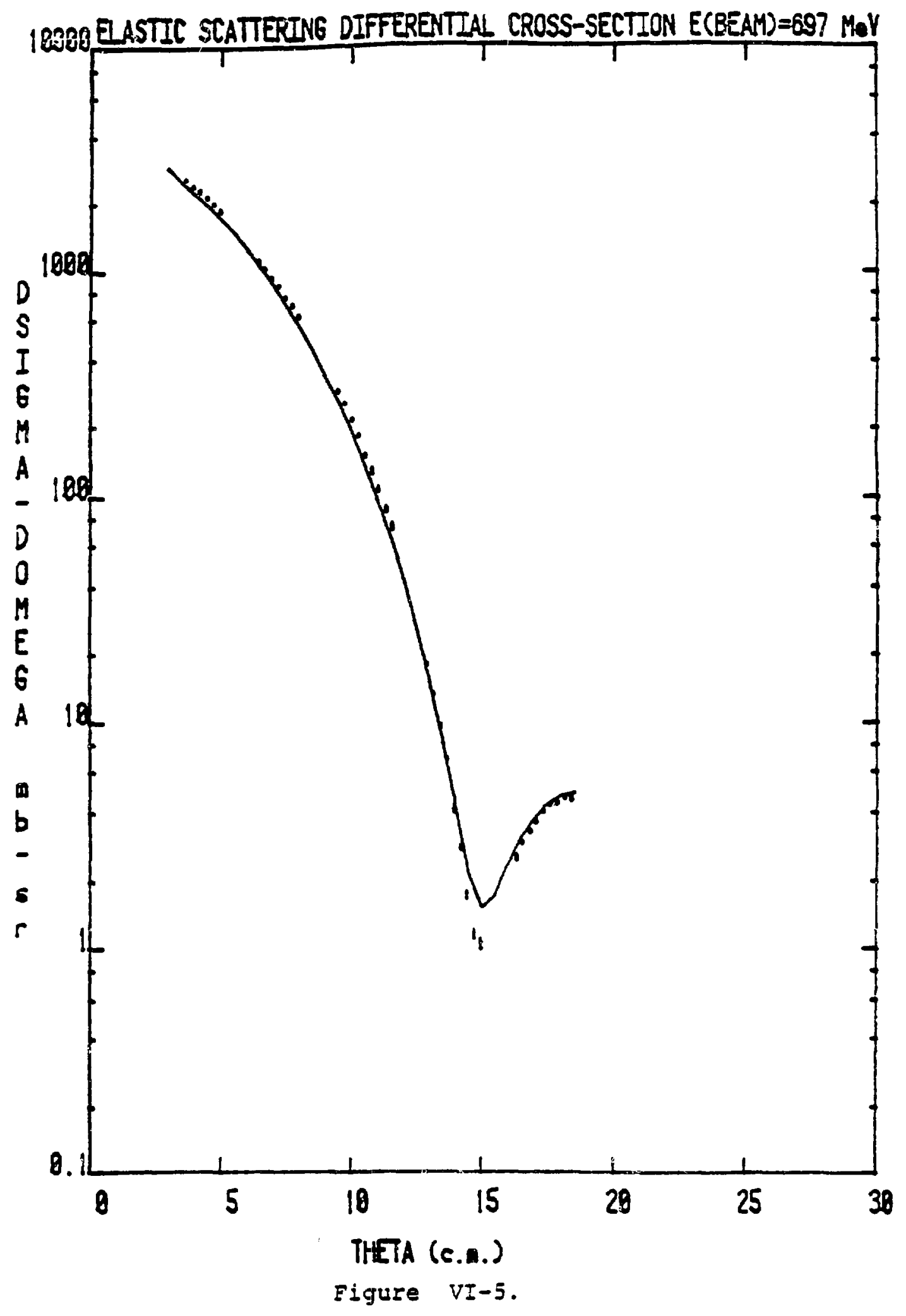




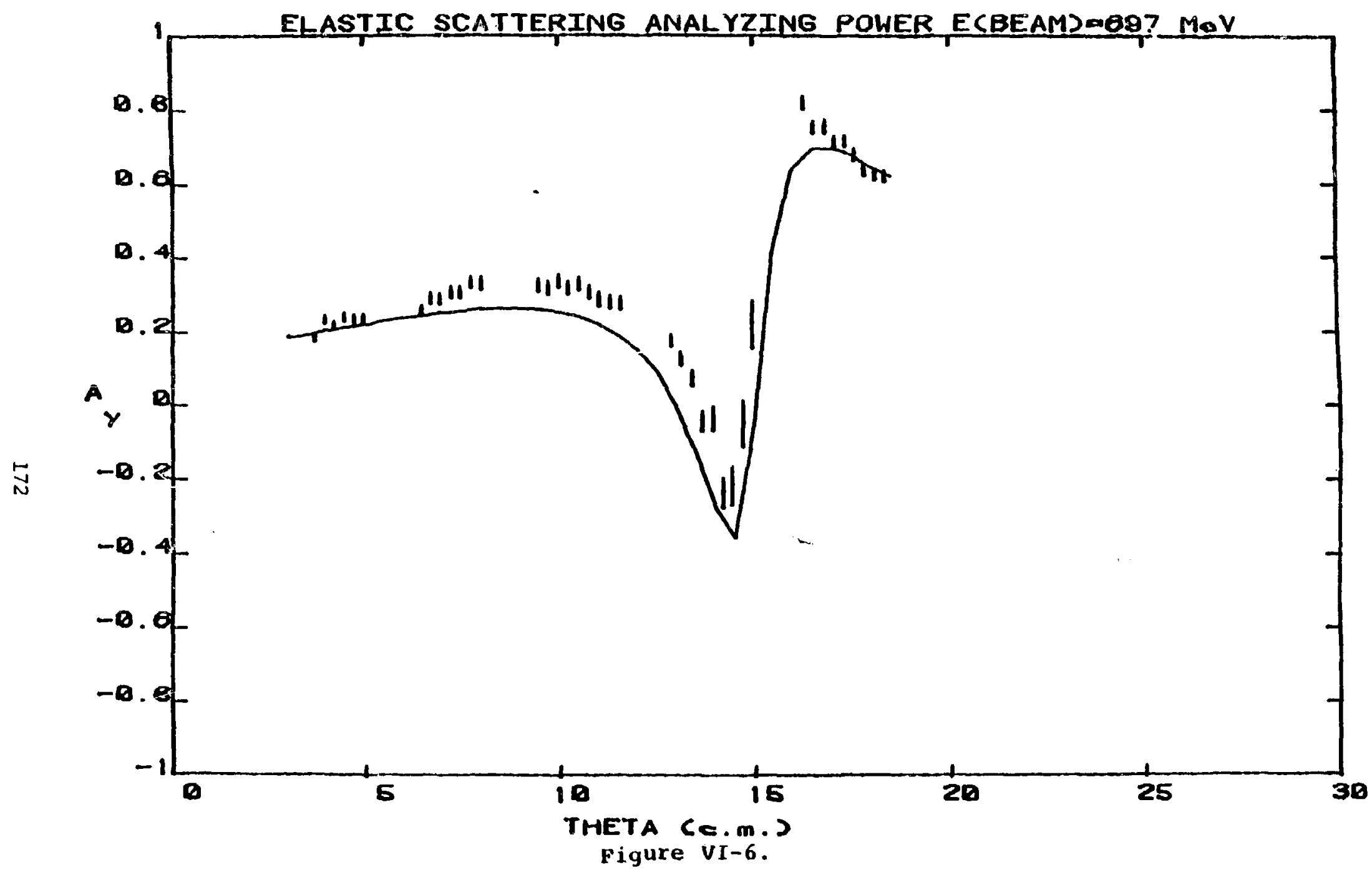


aithough the centrai imaginary strengths for 597 and 693 MeV were greater than that for $800 \mathrm{MeV}^{47}$ ). OE nore concern were the radius parameters of the weits, especiaily that of the reai central and surface imaginary terms. The real centraj radius exhibited wide variation from beam energy to beam energy, the vaiue of $2.53 \mathrm{fm}$ for $E_{p}=597$ Mev being rather unphysical; the surface imaginary radius was of the order of $1.7 \mathrm{fm}$. It is reasonabie to expect the real central radius to be representative of the physical size of the nucieus when muitiplied by $A^{(1 / 3)}$. The adjusted radius for $E_{p}=597 \mathrm{MeV}$ is then too large by a factor of two. Nevertheiess, attempts to constrain this parameter to a more reasonable value such as $1.2 \mathrm{fm}$ whlie adjusting the potential strength to maintain the volume integraj of the real centrai term at a constant vaiue were unsuccessful. Such a procedure caused a significant deterioration in the quality of the fit to the differentiai cross section. This is surprising since the stzength of this potentiai is so smail. We aiso note that the centrai imaginary radii for 597 and $698 \mathrm{MeV}$ were smalier than that for $800 \mathrm{MeV}$ by about 7 . This results in about a 228 decrease in volume which is consistent with the fact that the potentlals are stronger. Since these parameters were to be used solely as entrance and exit average potentials for the DWIA computations for ineiastic transitions, it was decided to retain the optimaj Elt parameters, and to run test DWIA calculations for a mora physicaliy realistic potential. Independent elimination of 
the imaginary surface term and the reai cencrai term was Eound to have no signiflcant effect on the resuits of the DWIA calculations. The ceibabibity of the normailization of the data indicates that misdetermination of the imaginary central term is aiso unlikeiy.

It is interesting to note that while the reai spinorbit potential is always attractive (negative in the CUPID convention), the reai central potential changes from being attractive at $\varepsilon_{p}=398 \mathrm{MeV}$ to repuistue for $E_{p}=597$ and 698 MeV. This is consistent with the fact that this term is repuisive at $800 \mathrm{MeV}$ incldent proton energy, and aiso with the observations noted in Chapter II. The largest variation In strength is seen for the imaginary central term, which increases by aimost a factor of four from $E_{p}=398$ MeV to $E_{p}$ = $698 \mathrm{MeV}$. The imaginary spin-orblt potentiai increases in strength by about a factor of 1.6 , while the reai spln-orbit potentiai decreases in magnitude by a factor of 1.7. The absoiute vaiue is then about constant. Note that this is also appioximateiy true of the $t_{0}^{i s}$ term in the Love-franey interaction.

B) Distorted Wave Inpuise Approximation Caiculations

The microscople DWIA calculations were performed using a modified verston of the code DWBA7048), known as DW8149). Since it 1 s expected that the impulse approximation is valid for incident beam energies greater than 400 
Mev50), the use of this approach for study of the Inelastic transitions was deemed appropriate. Furthermore, reactions were assumed to be single- step, and multipie scattering corrections were assumed to be adequately described by the distorted waves. Knock-on exchange calculations, which are of importance for some transitions, were performed exactiy, and relativistic kinematics was used throughout. The optical potentials used have been discussed in the preceding section of this chapter.

Harmonic oscillator radial wavefunctions wero used in all computations. The lp shell transition densities of Cohen and Rurath ${ }^{(7)}$ were used to describe states of positive parity. States of negative parity were described by the lp-ld, lp-ls and Ip-2s sheil transition densities of MiIlener ${ }^{18)}$. The harmonic osciliator length parameters for given transttions were chosen to match prominent maxima of the longltudinal and transverse form factors measured in inelastic electron scattering. Where such measurements do not exist, a length parameter of $1.681 \mathrm{fm}$ consistent with elastic scattering has been adopted. The transition densities used in the DWIA caiculations, together with osciliator length parameters appropriate to each transition and corrected for centre-of-mass effects as described by comfort ${ }^{6)}$, are given in Tabie VI-2. The effective interactions of Love and Franey ${ }^{3)}$ approprlate to nueleon-nueleus coli1sions at different energies were used throughout. The de- 
Reduced Matrix Elements

\begin{tabular}{|c|c|c|c|c|c|c|c|c|c|c|c|c|c|}
\hline$J^{\pi} ; T$ & $b(\mathrm{fm})$ & $p_{1} s_{1}$ & $p_{3} s_{1}$ & ${ }^{o}{ }_{1} p_{1}$ & $d_{3} p_{1}$ & $d_{5} p_{1}$ & ${ }^{a}{ }_{1} p_{3}$ & $d_{3} p_{3}$ & $d_{5} p_{3}$ & ${ }^{p}{ }_{1} p_{1}$ & $\mathbf{p}_{1} \mathbf{p}_{3}$ & $p_{3} p_{1}$ & $p_{3} p_{3}$ \\
\hline $1^{+} ; 0$ & 1.572 & & & & & & & & & -.0434 & .7188 & .3544 & .0137 \\
\hline $2^{+} ; 0$ & 1.681 & & & & & & & & & & $.7594-$ & $-.5031-$ & -.3100 \\
\hline $1^{+} ; 1$ & 1.866 & & & & & & & & & -.0581 & $-.6901-$ & $-.3394-$ & -.0764 \\
\hline $2^{+} ; 1$ & 1.570 & & & & & & & & & & .6801 & -.1232 & .0608 \\
\hline $2^{+} ; 10$ & 21.570 & & & & & & & & & & .4871 & -.0131 & .1327 \\
\hline $1^{-} ; 0$ & 1.681 & .0543 & -.0436 & .2701 & .0999 & & .6306 & -.1219 & .3195 & & & & \\
\hline $2^{-} ; 0$ & " & & -.0199 & & $-.0710-$ & -.1216 & $5-.3956$ & .1639 & -.3875 & & & & \\
\hline $2^{-} ; 0$ & $"$ & & .0107 & & -.0144 & .0924 & .2783 & .1189 & -.6988 & & & & \\
\hline $2^{-} ; 0$ & $"$ & & -.0440 & & .1160 & .1309 & -.5969 & -.1459 & -.0594 & & & & \\
\hline $3^{-} ; 0$ & $"$ & & & & & .2654 & & -.3145 & .5099 & & & & \\
\hline $4^{-} ; 0$ & $"$ & & & & & & & & .3390 & & & & \\
\hline $1^{-} ; 1$ & $"$ & -.0842 & .0235 & .1073 & .0031 & & -.7172 & 2.1199 &.- .2773 & & & & \\
\hline $1^{-} ; 1$ & $"$ & -.0283 & $-.0216-$ & -.5280 & .0021 & & -.0947 & -.0150 & -.0652 & & & & \\
\hline $2^{-} ; 1$ & " & & .0133 & & -.0332 & .0047 & 7.7079 & -.0050 & .3474 & & & & \\
\hline $2^{-} ; 1$ & $"$ & & -.0074 & & -.0678 & -.2973 & $3-.3213$ & $3-.0904$ & .5521 & & & & \\
\hline $3^{-} ; 1$ & $"$ & & & & & -.0384 & & -.1322 & $2-.7510$ & & & & \\
\hline $4^{-} ; 1$ & $"$ & & & & & & & & -.8069 & & & & \\
\hline
\end{tabular}

Table VI-2. 
taijs of the force have been summatized in Chapter II of this work. The $425 \mathrm{MeV}$ interaction was used for calculations to be compared with the 398 MeV data, while the 650 MeV interaction was used for calcuiations to be compared with the 597 and $698 \mathrm{MeV}$ data. On occaston the $515 \mathrm{MeV}$ force was used for comparisons with the $597 \mathrm{MeV}$ data, as was the 800 MeV force for comparisons wlth the 698 MeV data. Aij caicuiatlons were performed trcluding exchange. The resuits are presented in the form of six sets of curves for each transtion. These curves are getierated by sequential addition of the resuits for reai and imaginary components of the force with approprlate phases. A key which tiay be used in the interpretation of the varlous curves is given in Figure VI-7. The symbols $C$, LS and $T$ denote the contral, spin-orb:t and tensor components of the force, while the symuss $R$ and $I$ denote real and inaginary parts.

In crder to provide a broader setting for both the data and results of the computations, we discuss for each transition not only the results of our work, but those obtained by other authors at other incldent proton energies. It is hoped that such a systematis study wiil provide a broader understanding of the transitions investigated. Where applicable, results of studies using probes other than protons are discussed, Comparlson with electron scattering is very useful. Even if the transition densities are not weil determined, useful information concerning the nucieon- 
Key for Curves in Figures VI-8 to VI-56

Comparisons with Data

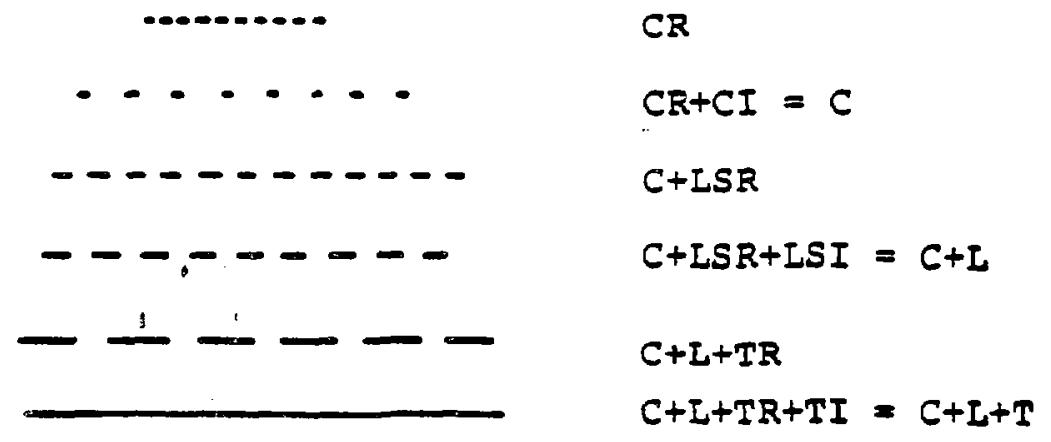

Comparison of Calculations at cifferent Energies $\Sigma_{p} \quad$ Force $398 \mathrm{MeV}(425 \mathrm{MeV})$ $597 \mathrm{MeV}(515 \mathrm{MeV})$

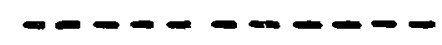
$597 \mathrm{MeV}(650 \mathrm{MeV})$ $698 \mathrm{MeV}(650 \mathrm{MeV})$ $698 \mathrm{MeV}(800 \mathrm{MeV})$

Figure VI-7. 
nucleon interaction and reaction dynamics may be extracted by comparing nomalization factors necessary to bring ineIastic eiectron and proton scattering caicuiations into agreement with the data. If these renormaitzation factors are comparable, then it is approprlate to say that the description of the ( $\left.P, P^{\prime}\right)$ interaction and reacticin dynamies is reasonable and the difficulties lie with the transition densities.

1) Isoscalat states of natural partity

(d) The $2_{I}^{+} ; 0$ state at $\varepsilon_{x}=4.44 \mathrm{MeV}$

The dominant configuration for the excltation of this state is $\left(P_{1 / 2} P_{3 / 2}^{-1}\right)$. The $\left(P_{3 / 2} P_{1 / 2}^{-1}\right)$ conflguration ytelds an almost comparable contribution of opposite phasing, and the $\left(P_{3 / 2} p_{3 / 2}^{-1}\right)$ term is half as strong as rne $\left(p_{1 / 2} p_{3 / 2}^{-1}\right)$ term and also of opposite phase. The osciilator parameter used was $1.681 \mathrm{fm}$.

The resuits of the calcuiations are presented in Figures VI-8 to VI-11. The calculations are seen to significantiy underestimate the data for all bean energies. This is not unusual for natural parity coliective states where effective charges are needed to explain $B(E L)$ values. Renormalization factors of $2.10,3.25$, and 3.74 are required to produce the agreement shown in Figure VI-11. With this 

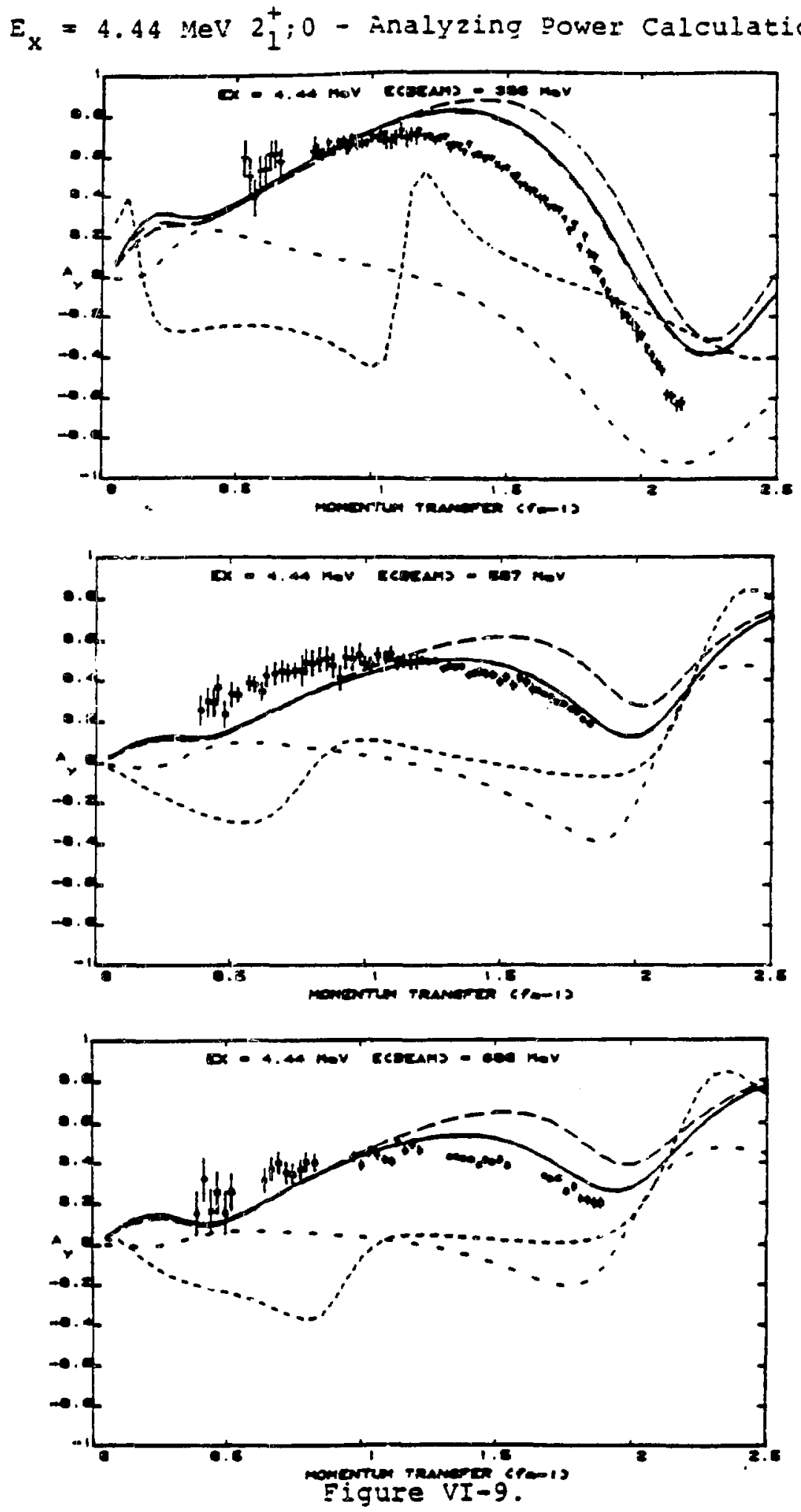
$E_{x}=4.44 M e V 2+i 0-$ Renormalized Differential Cross Sections
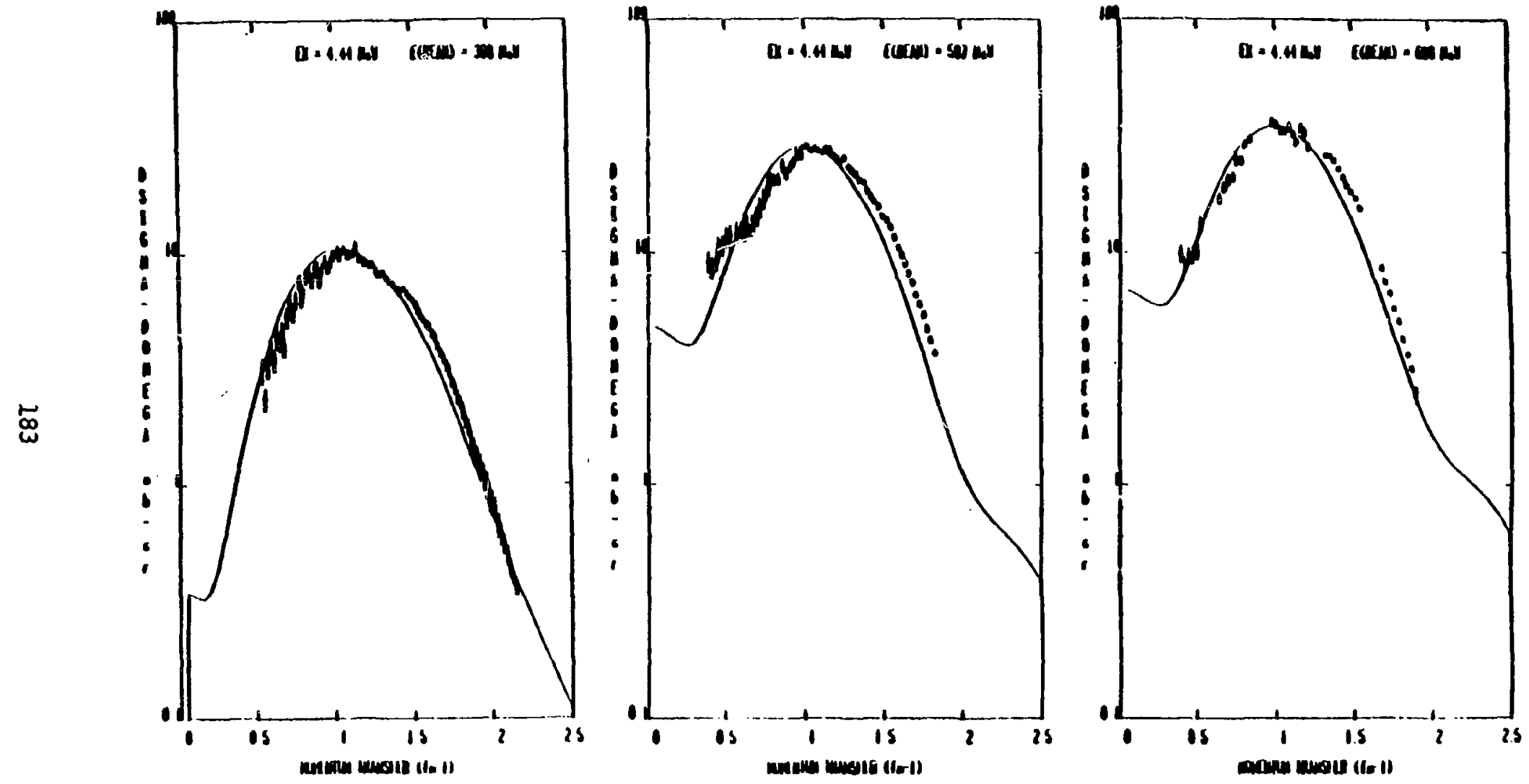

Figure VI-11. 
correction the quaity of the fit is good but not perfect. The calculated cross section overestimates the data over the reglon encompasing the rise to the maximum, while underestimating the data in the region of fall-off for $q>1 \mathrm{fm}^{-1}$. The problem of understanding the large momentun transfer data has been reported by comfort et. aj. $5-7,9)$ for incident energies from 122 MeV to 200 MeV whers iarge monentur transfer fits are much worse than shown here. Densitydependent modificatians to the interaction determined by Keily et. ai. ${ }^{51)}$ for isosealar natural partty transitions yielded considerable improvement in the shape of the differential cross sections and the absolute cross section scaie for fits to the $122 \mathrm{MeV} \operatorname{data}^{52}$. Such effects ars expected to be much smaller at our energies. From figurs VI11 It is apparent that a smali adjustment to the oseiliator parameter would give a very good fit of the renormalized calculation to the data. Coupled channels effects have been seen to contribute to enhancements of calculated cross sections for large $q$. Such lnvestigations are currentiy being conducted and will be reported eisewhere.

The renormalization factors are of considerabie fnterest. The electromagnetic excitation of this transition in inelastic electron scattering indicates that core polarization effects result in an enhancement of the pure CohenKurath results by a factor of two 53,54$)$. Aiso, the experimental $B(E 2)$ value exceed 3 the predicted vaiue by about 2.o. 
Consequently, it is reasonable to require that the results of the DWIA calculations be multiplied by 2.0 for comparison with the data. The calculation at $398 \mathrm{MeV}$ after renormallzation to account for such effects differs from the data only by some 108. From Table VI-3 we see that addttionaI enhancement factors of 1.55 and 1.75 are required after renormalization to bring the calculations for 597 MeV and 698 Mev into agreement with the data. Comfort et. aj. 5-9) have seen that sultably renormalfzed fits to data at 122,185 and $200 \mathrm{MeV}$ consistentiy overestimate the data by factors of 1.15 to 1.9. Haj1-Saied et. ai. ${ }^{15)}$ report reasonable agreement with the data of Blanpled at $800 \mathrm{MeV}$ after appropriate renormalization. They observe an overestimation for small angles and an underestimation for $q$ between 1 and $2 \mathrm{fm}^{-1}$, as is found in the current work. It is not ciear why the progression from overestimation at low incident energies to underestimation at $598 \mathrm{MeV}$ is broken for the 800 MeV data. Medium cosrections have not been applied to any computations for energies greater than $122 \mathrm{MeV}$, and while these corrections are vory successful at $122 \mathrm{Mev}^{52)}$, it would be interesting to see if such successes can be repeated for higher energies where deficiencies are seen to exist at large momentum transfer.

Carefui observation of the curves in Figure VI-8 show the primary dependence of the cross section on the imaginary central part of the force. This is different from 
the observations of comfort et. ai. ${ }^{9}$ ) who find that at 200 MeV the transition is dominated by the reai part of the central Interaction. Removal of the imaginary term for these lower energy calcuiations is seen to make a small difference and results in an improvement in the quality of the flt to the zross section, but results in a degradation of the fit to the analyzing power. The difference in dependence on real and lmaginary parts of the central interaction is understood in terms of the energy dependence of the real and imaginary strengths of the central optical potential described previousiy. Enhancement due to the spin-orbit component of the force is significant, especialiy at large momentum transfers, while the tensor terms play essentialiy no role. Such observations are in keeping with the sxpectations outlined at the end of Chapter II. Calculations with the $515 \mathrm{MeV}$ force required an increase of 1.4 in the renormailization factor required to reproduce the $597 \mathrm{MeV}$ data, showing poorer agreement. The calculation with the $800 \mathrm{MeV}$ force reduced the renormalization factor for the $698 \mathrm{MeV}$ data by 1.4 , resulting in better agreement with the data.

Turning to the analyzing powers, we see from Figure VI-g that the shapes of $A_{Y}$ for the different beam energles are reasonably well reproduced, but a phase difference between caiculation and data is clearly evident. Forward. angle data are generaliy underestimated, while larger angle dara are overestimated. A similar phase difference has been 
observed by Comfort et. ai. at $200 \mathrm{MeV}^{9}$, while calcuiations at 120 MeV are found to overestimate the data uniformiy ${ }^{6)}$. The microscoplc spin-orbit interaction is seen to be primarily responsible for reproducing the shape of the anaiyzing powers. The mismatch between data and calculation for the analyzing power alscussed here does, not mirror that seen for the elastic analyzing power.

It is appropriate to consider the roie played by spin-orbit distortions in the incoming and outgoing channeis. Computations were performed with the spin-orbit optical potential terms set to zero. The centrai force yields zero anaiyzing power, but the microscopic spin-orbit force was found to reasonaily reproduce the shape of Ay for $q<1$ $\mathrm{fm}^{-1}$. The analyzing power so calculated was in generai larger than that computed with distortions, and the structure seen between 2 and $2.5 \mathrm{fm}^{-1}$ was cemoved. The calculated cross section was reduced by about $12 \%$, resulting in a comparable increase in the renormalization factor. Spin-orbit distortion effects are therefore seen to piay a relatively minor roie for low momentum transfers, but are signiflcant for $q>$. . . f $\mathrm{Em}^{-1}$.

The effect of inciuding knock-on exchange in the caicuiation is to stgniftcantly reduce the calculated cross sections, and to enhance the analyzing powers. The interference of the direct and exchange terms in the centrai imaginary calculation results in a reduction of the direct 
cross section by a factor of almost 3.5, while enhancing the anaiyzing power computed in the spin-orbit terms by a factor of 2 .

The qualltatlve features of $\sigma * A_{y}$ are reproduced by the calculations. However, the $698 \mathrm{MeV}$ result is enhanced above that jor $597 \mathrm{MeV}$, contrary to the behaviour of the data.

(b) The $3 ; ; 0$ state at $E_{x}=9.64 \mathrm{MeV}$

The dominant configuration for the excitation of this state is $\left(d_{5 / 2} p_{3 / 2}^{-1}\right)$, with slgnifleant contributions from $\left(d_{5 / 2} P_{1 / 2}^{-1}\right)$ with the same phasing and $\left(d_{3 / 2} P_{1 / 2}^{-1}\right)$ with opposite phasing. The osciliator parameter used was 1.681 fm.

The results of the calculations are presented in Figures VI-12 to VI-15. As was the case for the $2_{1}^{+} ; 0$ state, the calculations significantiy underestimate the data. Renormalization factors of $1.85,2.93$ and 3.60 are required to produce the good agreements shown in Figure VI-15. Some over- estimation by the calculations for low $q$ after renormalization is soen for 597 and $698 \mathrm{MeV}$, but agreement for $398 \mathrm{MeV}$ is excelient. Table VI-3 shows that the enhancement factors for 597 and $698 \mathrm{MeV}$ relative to $398 \mathrm{MeV}$ are 1.58 and 1.62 respectively, in reasonabie agreement with those determined for the 2 io state. Comfort et. al. Elnd that ifteie renormalization is required for this transition for $E_{p}=208$ 
$E_{x}=9.64 \mathrm{MeV} 3_{1}^{-} ; 0$ - Differential Cross Section Calculations
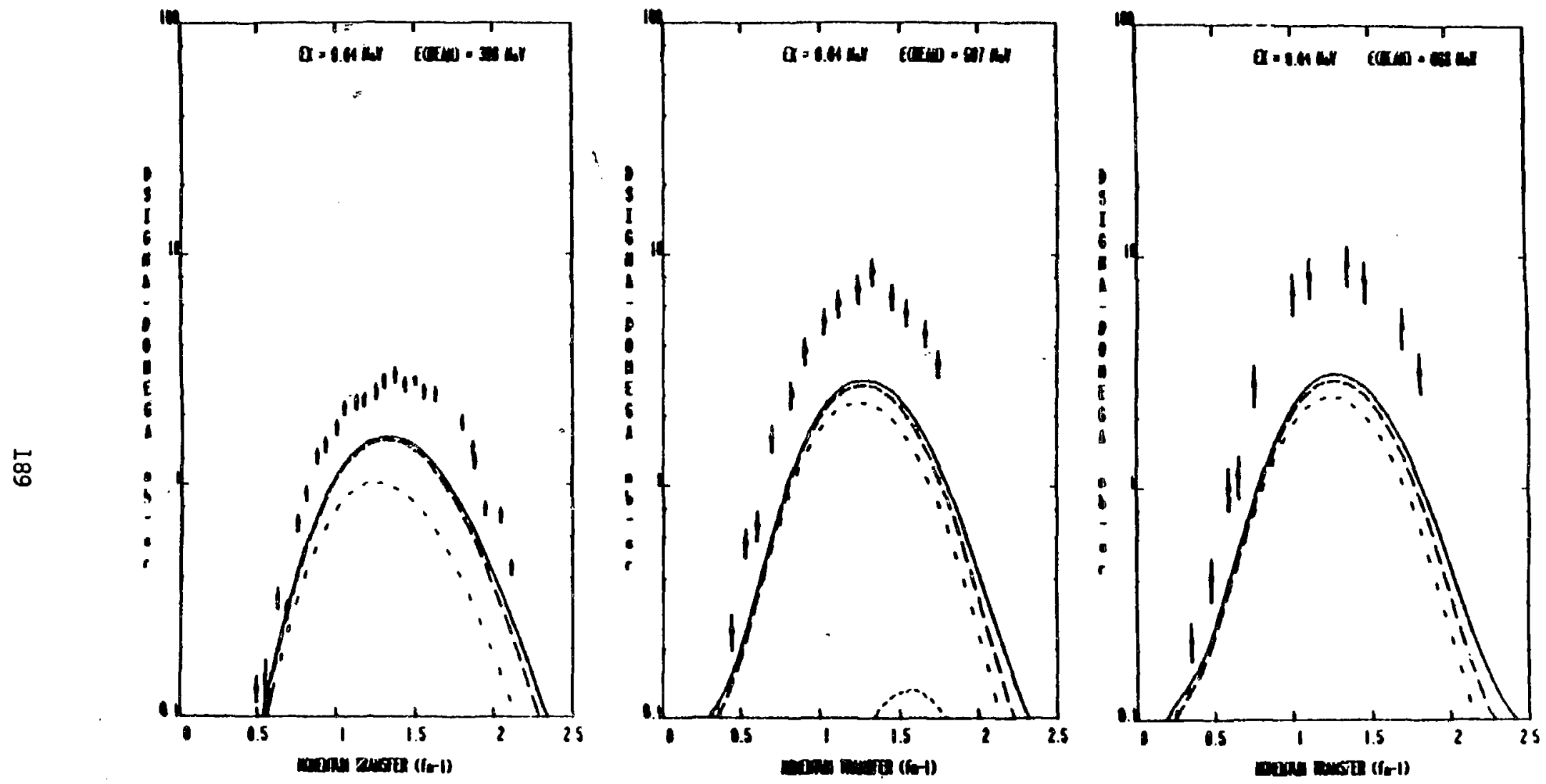

Figure VI-12. 
$E_{X}=9.64 \mathrm{MeV} \mathrm{3}{ }_{1} ; 0$ - Analyzing Power Calculations
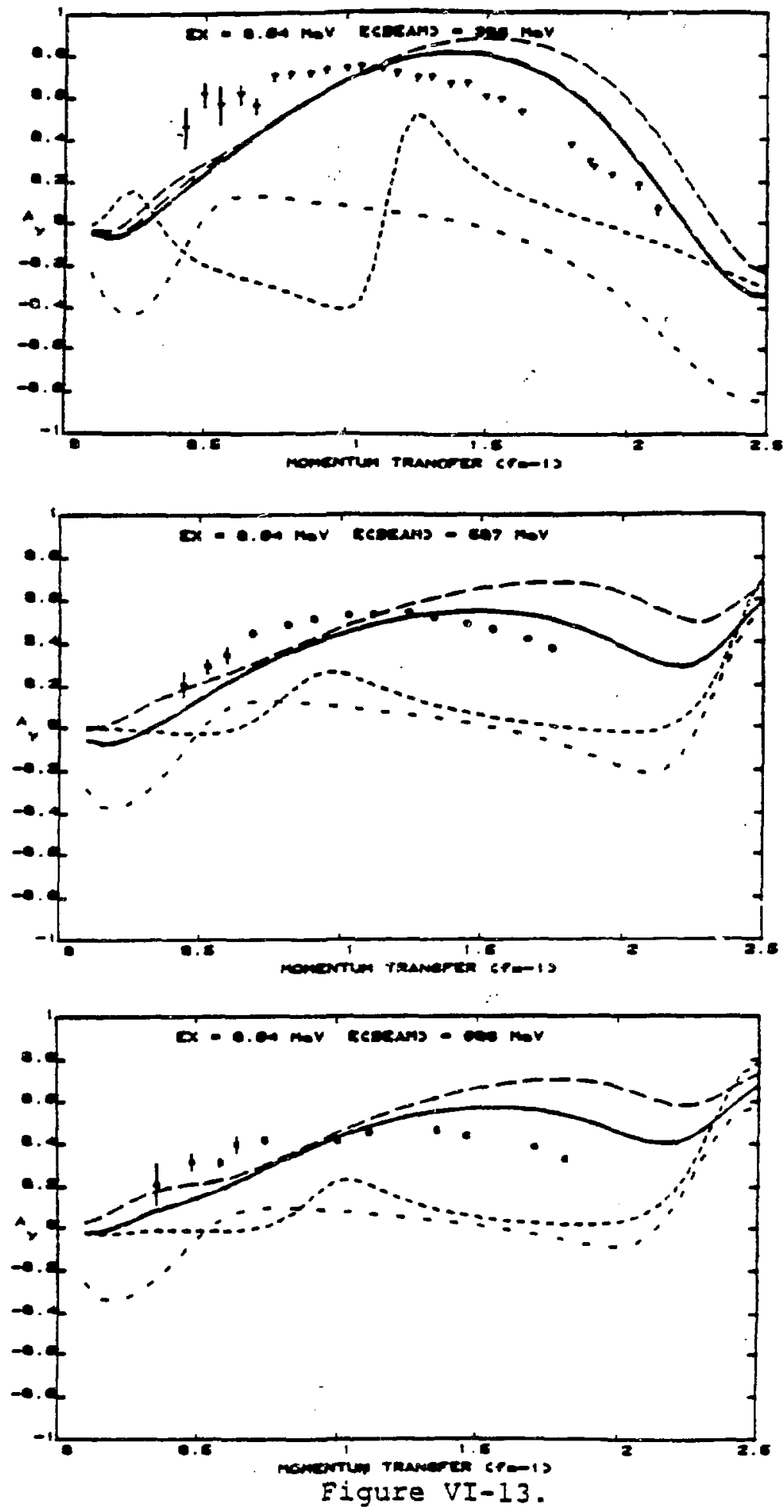
$E_{x}=9.64 \mathrm{MeV} 3_{1}^{-} ; 0$ - Comparative Calculations for Observables
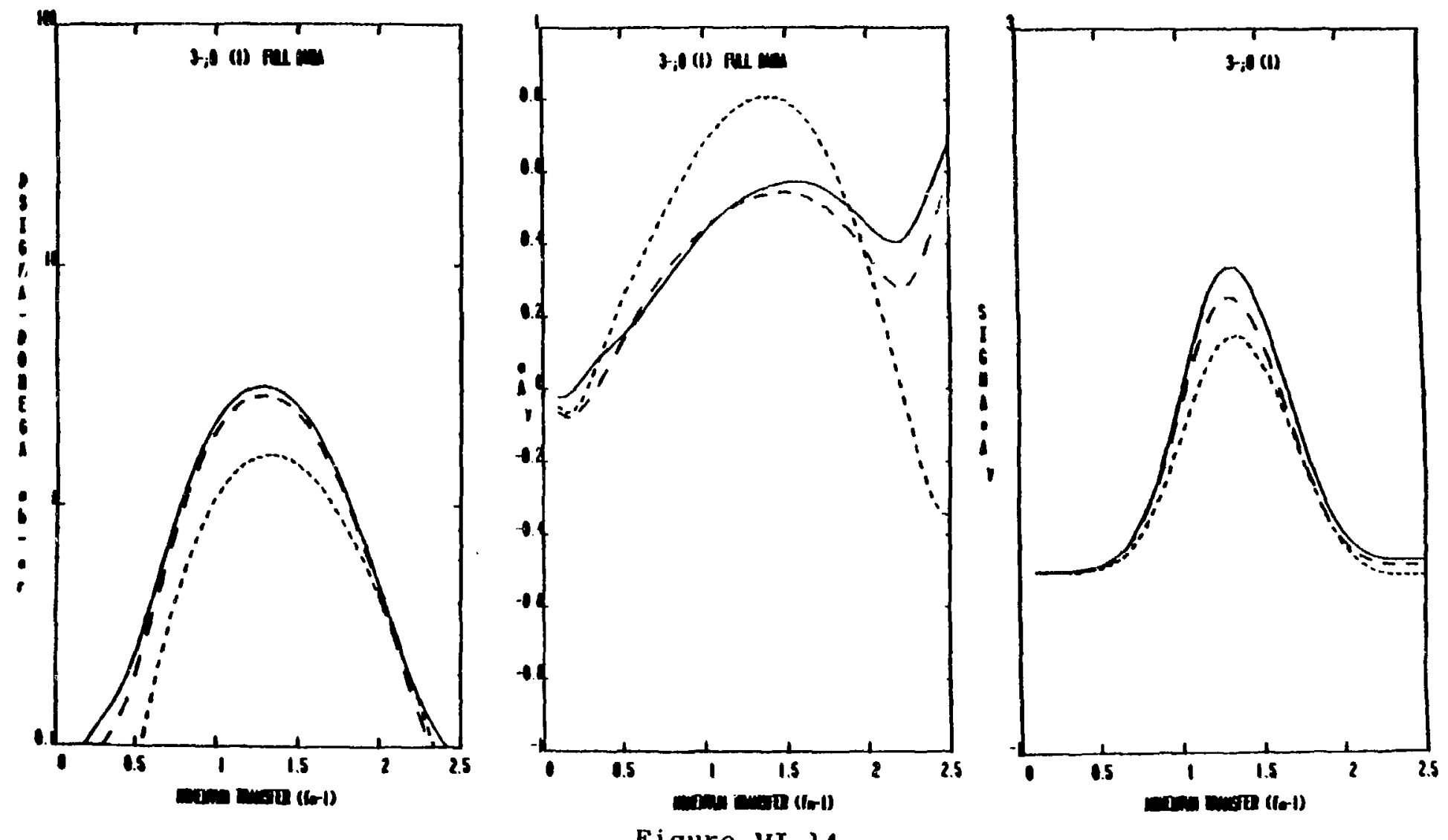

Figure VI-14. 
$E_{x}=9.64 \mathrm{MeV} 3 \overline{1} ; 0$ - Renormalized Differential Cross Sections
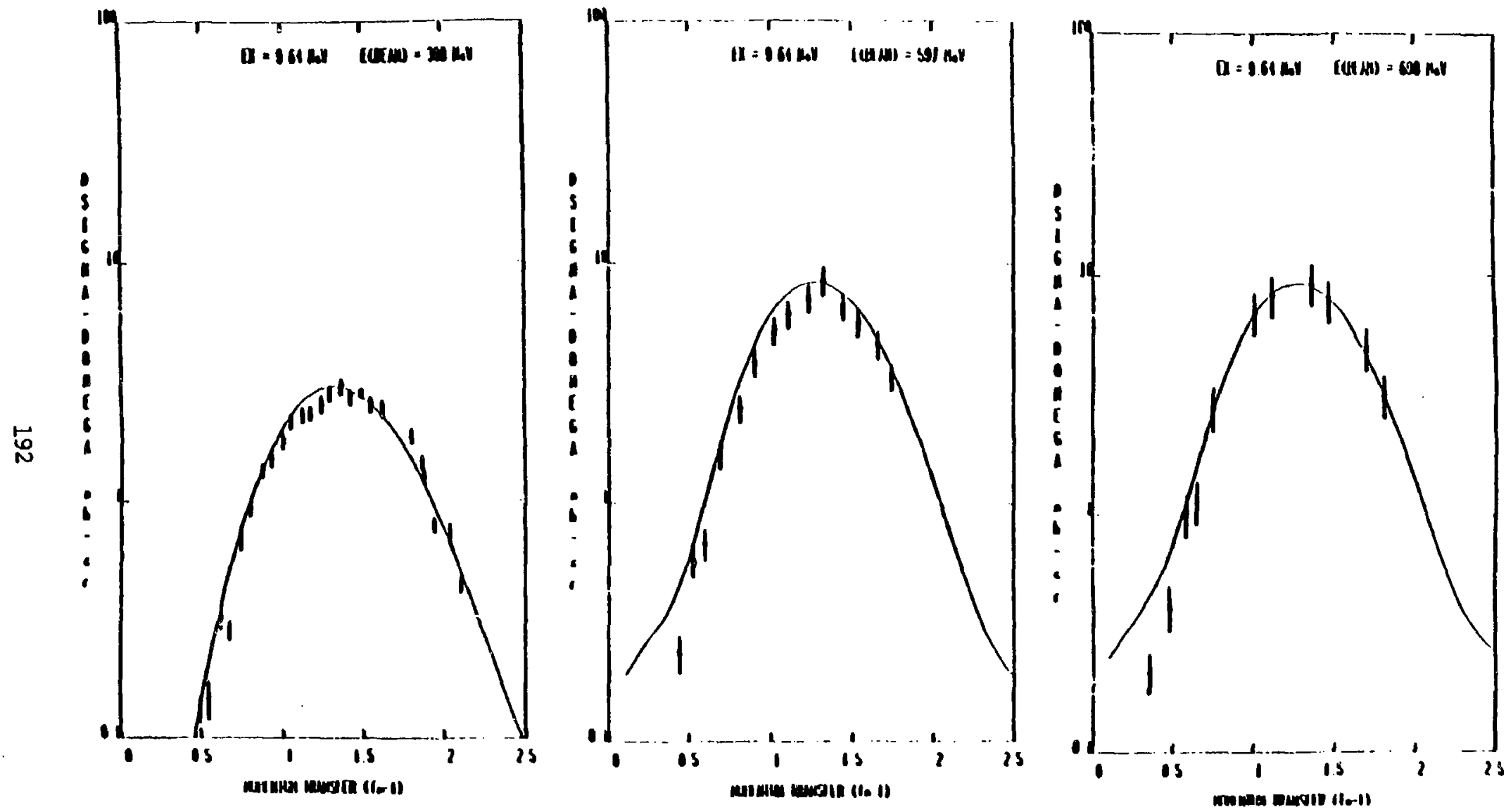

Figure VI-15. 
$\mathrm{MeV}^{91}$.

The dominant longitudinal eiectron scattering form Eactor is well. reproduced when renozmalized by a factor of two out to a momentum transfer of about $1.7 \mathrm{fm}^{-1}$, and the weaker transverse form factor is underestimated by about a factor of $0.6^{9,54)}$. A renormalizaton by about two of our calculations is therefore justifled, and so agreement at 398 MeV is reasonable. Comfort et. al. also found little ssnst.tuity to the $S=1$ amplitudes in the wavefunctions ${ }^{9}$ ).

Study of the curves in figure VI-12 show the primary contributions to the cross section to come from the central imaginary and real spin-orblt terms in the interaction, the spin-orblt term providing enhancement primarily in the high $q$ region and at $398 \mathrm{MeV}$. The relative enhancement due to this term is seen to decrease for increasing incident ener9y. The strong dependence on the imaginary part of the interaction is again opposite to that observed by Comfort et. al. who find the primary contribution to come from the real central term ${ }^{9}$. Medium corrections have not been considered for this transition, and coupled channels effects are currenty under investigation.

The analyzing powers, as seen in Flgure VI-13, are reasonably well reproduced in shape, but extribit a problem in phasing similar to that discussed in the previous section. Forward angle data are underestimated while large 
momentum transfer data are overestimated. Again the domInan contribution to this observable is the real spln-orbit term in the interaction. A slight phase difference in the sense observed in this work is found to exist for the data at $200 \mathrm{MeV}^{91}$.

Removing spin-orbit distortion effects in the optical potential results in effects almost 1dentical to those reported in the prevlous section. knock-on exchange ampl1tudes again reduce the direct amplitude cross sections by a factor of 3.2, and in this instance the analyzing powers are also enhanced by the exchange effects. The qualitative features of the energy dependence of the calculared crose sections are congistent with the energy depandence of the measured cross sections. The shape of the product $\sigma{ }^{*} A_{y}$ is consistent with the data, but the difference in Ay yieids a greater difference between the calculated values for 597 and $698 \mathrm{MeV}$ than is seen for the ata.

(c) The $1_{1}^{-}$;0 state at $E_{X}=10.84 \mathrm{MeV}$

The dominant configuration in the transition density is $\left(2 s_{12}{ }^{1 p} \overline{3} \frac{1}{2}\right)$ with additional signifleant contributions from the $\left(d_{5 / 2} p_{3 / 2}^{-1}\right)$ and $\left(2 s_{1 / 2} 1 p_{1 / 2}^{-1}\right)$ terms, which are of the same phase as the dominant component. Small contributions Erom a varlety of other configurations are present, and may be found in Table VI-2. The oselilator parameter used was $1.681 \mathrm{fm}$. 
The resuits of the calcuiations are presented in Figures VI-16 to VI-19. The fit to the cross section data for an incident energy of $398 \mathrm{MeV}$ is remarkabiy good, while the data for $597 \mathrm{MeV}$ are underestimated by a factor of 1.83, somewhat larger than the $2^{+}$and $3^{-}$states. The fact that the shape of the data is so well fitted is especialiy interesting in view of the fact that it is very similar to the shape of the $3^{-}$data. A collective model would thus give a very poor fit. The paucity of data for 698 Mev precludes any statement concersing the relationship between daca and theory.

We note once more the strong contributions from the imaginary central component of the interaction, as well as the enhancement due to the spin-orbit term. It is of interest to note that the spin-orblt enhancement is now almost uniform as a function of $q$, rather than simply being most significant for large momentum transfer as observed for the two states discussed previously. It is surprising that the caiculated cross sectlons do not exhibit a clear systematic increasing trond as a function of incident projectile energy as seen in Figure VI-18. Indeed, the values for the diffarential cross section at the peak are almost ldentical for all beam energies. Some structure is seen at both very small and large momentum transfers.

The calculated analyzing powers exhibit some of the same qualitative features as those for the 4.44 and $9.64 \mathrm{MeV}$ 
$E_{x}=10.84 \mathrm{MeV} 1_{1}^{-} ; 0$ - Differential Cross Section Calculations
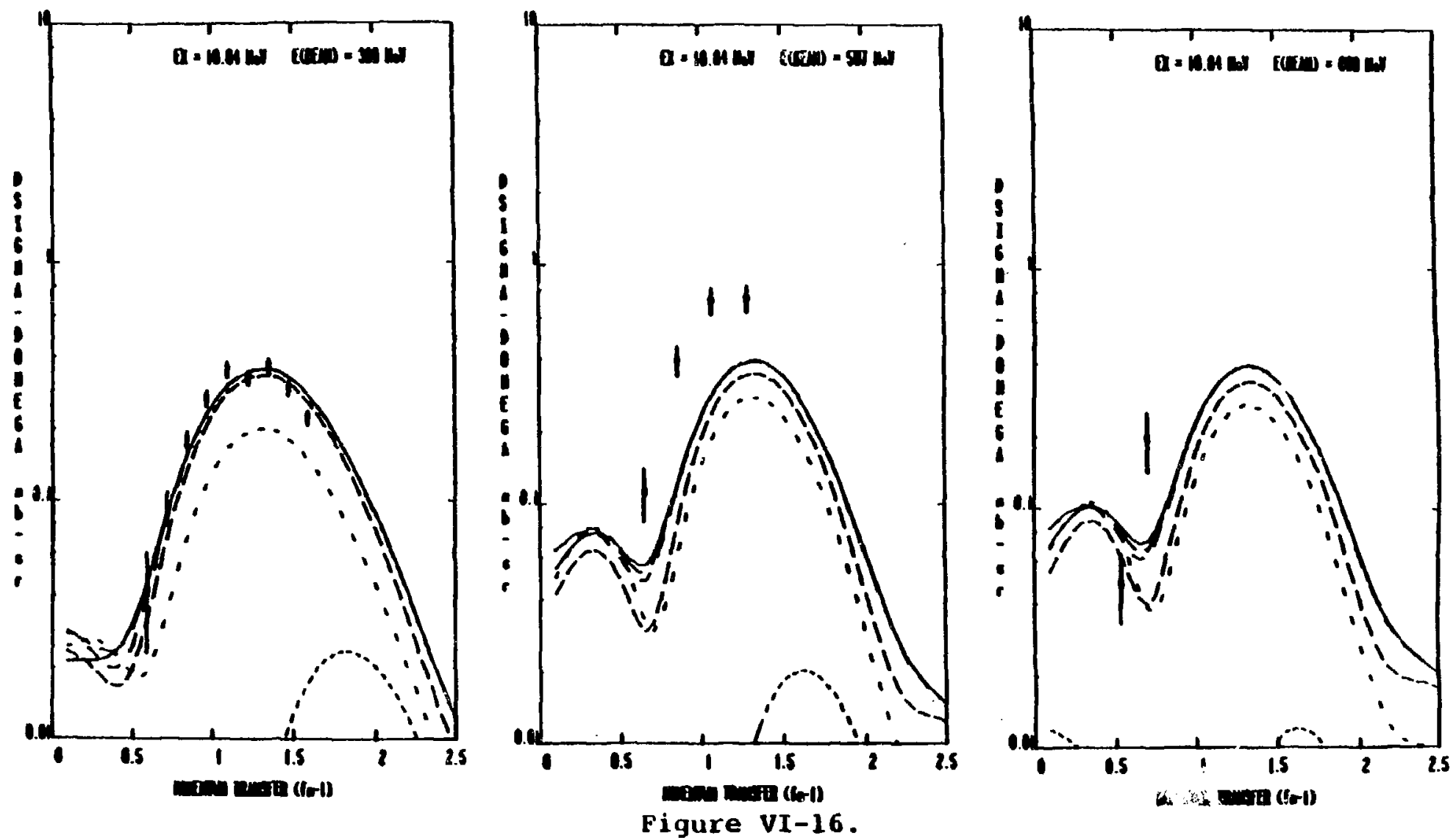

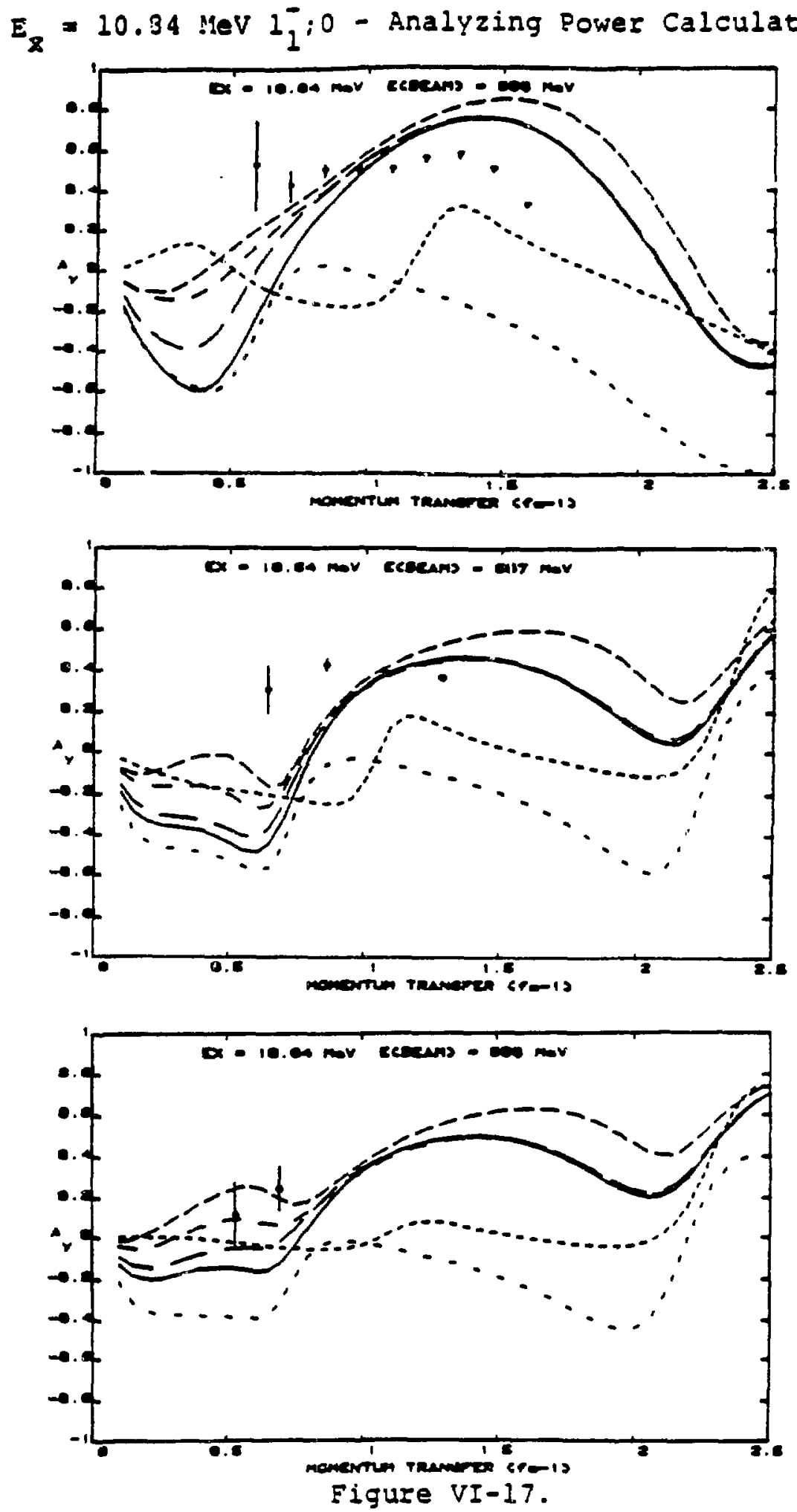
$E_{x}=10.84 \mathrm{MeV} 1_{1}^{-} ; 0$ - Comparative Calculations for Observables
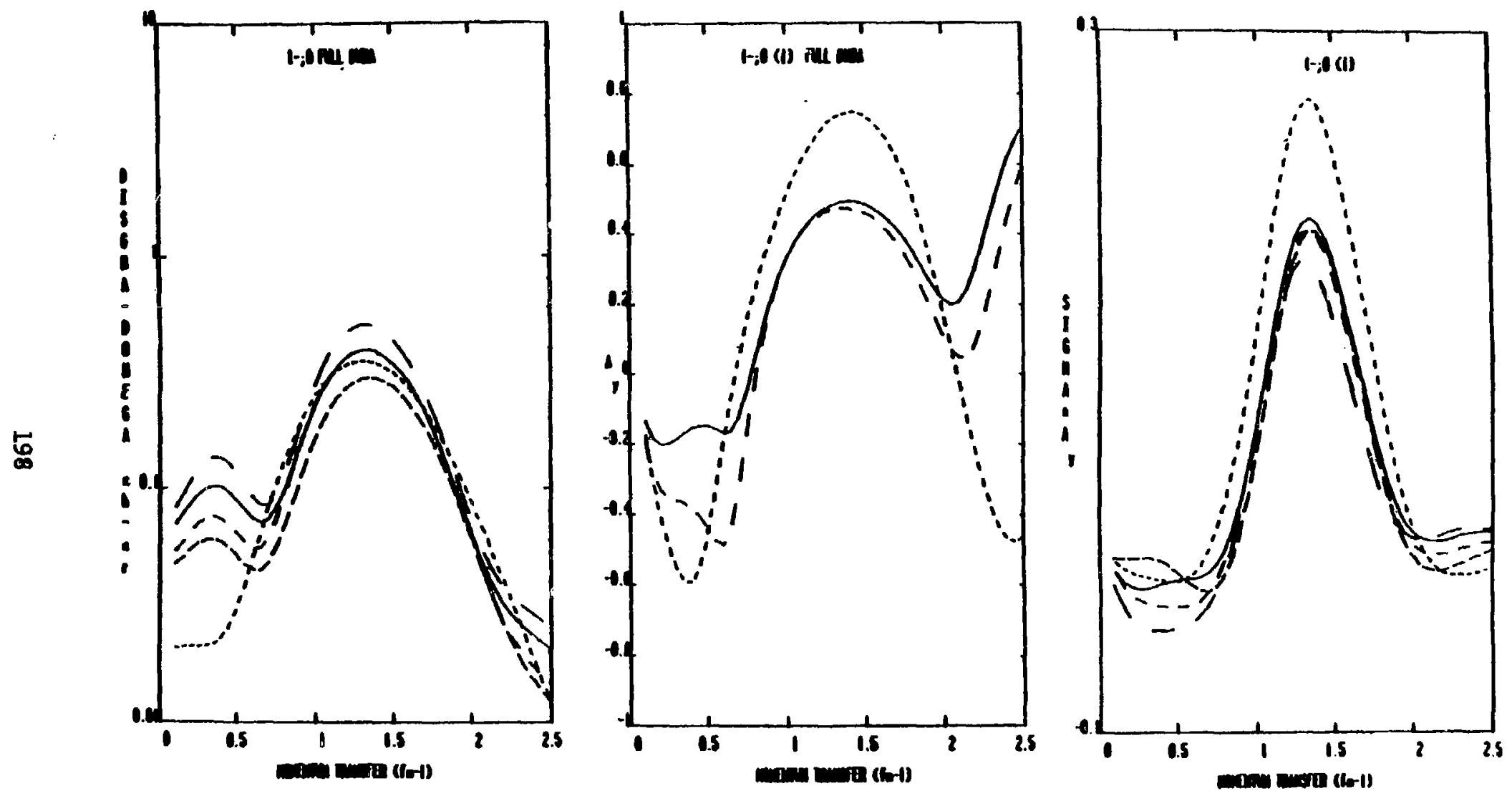

Figure VI-18. 


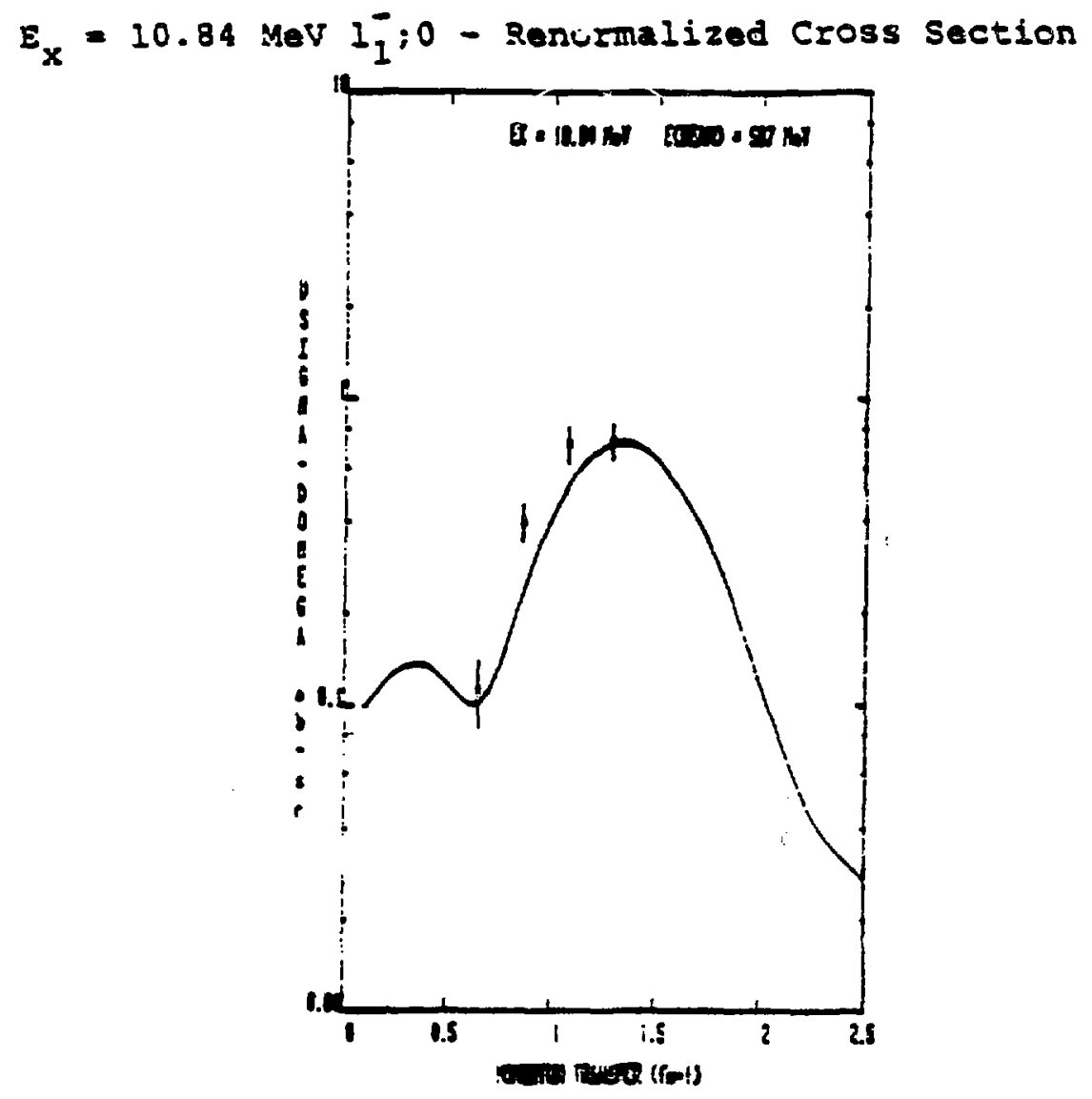

Sigure VI-19. 
states whlch have been discussed previousiy. There are some notabie differences, however, particularly in the low momentum transfer reglon from 0.0 to $0.6 \mathrm{fm}^{-1}$. Whilo the $2_{1}^{+}, 0$ and $3 \frac{-0}{1} 0$ states exhibit littie structure in this region, the cajcuiations for the $1_{1}^{-} ; 0$ state show pronounced negative minima. The depth of the minimum decreases with increasing projectile energy and at $597 \mathrm{MeV}$ two small mintma are seen to emerge. In addition, the tersor term is seen to play a signifleant role in this region, while intermediate and high monentun transfer data are dominated by the spin-orblt component of the interaction as for other isoscalar natural parity states. However, the small cross sectlon at low $q$ precludes observation of this effect.

The product of the differential cross section and anaiyzing power, while exhiblting the sharp peak characteristic of the natural parity lsoscalar transltions, shows an unusual trend. Whereas the $2_{1}^{+} ; 0$ and $3_{1}^{-} ; 0$ states show an increase in this quantity as a function of energy, this state shows a decrease, largeiy due to the sharp decrease in Ay from an incident energy of $398 \mathrm{MeV}$ to $597 \mathrm{MeV}$, glven that the differantial cross section is roughiy constant. In this context it is unfortunate that Ilttie data was extracted at 597 and 698 MeV, but as was seen in Figure V-18 indications are that the experimentaliy observed energy dependence of this quantity is not consistent with the calculations. Experiments at other energios have not reported data for this 
cransition, not have studies of this nueleus with other probes.

The substantive difference between this transition and the $2_{1}^{+} ; 0$ and $3_{1}^{-} ; 0$ transitions may be found in the transition densities. While the preceding two transitions involve particle-hole configurations exclusiveiy within the lowest (1) peincipal quantum number harmonic osciliator shell, the excitation of the $I_{j} ; 0$ trangition $1 \mathrm{~s}$ dominated by conflgurations requifing the promotion of a particle from the lowest to the second (2) shell. The $2 s_{1 / 2}$ harmonic ascillator wave function then necessarily has a node at about $I=2 \mathrm{fm}^{-1}$ and is negative for values of $\mathrm{I}$ smaller than this because the DWBI code requires the radial wave function to be positive at infinity. This then can result in differing sensitivities of the spin-orbit and tensor components of the interaction to the transition densities. Additional data for this transition would be most useful in examining this phenomenon.

Once again, littie dependence is seen on the distortion terms in the optical potentiais; the low momentum trangfer dependence beling relatively independent of these effecis. Knock-on exchange results in a reduction in the magnitude of the direct differential cross section by factor of 3.5, consistent with the other transitions of this kind. 


\section{(d) Summary}

We have determined several systematic trends for isoscaiar states of natural payity. The excitation of these states is dominated by the imfginary centrai and reai spinorbit components of the effective interaction. The dependence on the imaglnary part of the central term is different from the observations of Comfort et. aj. at iower energies. The differential cross sections were in general underestimated by the calculations. Spin-orbit distortion effects in the optical potentials were found to fiay a ininor role for Iow momentum transfers, but contributed significantiy to a reduction in $A_{y}$ for moderate values of $q$. Rnock-on exchange effects, which wer lncluded in all cases, were found to consistentiy interfere destructively with the direct processes, resulting in a reduction in the magnitudes of the caicuiated cross sections by a consistent factor of 3.5 , independent of energy. The anaiyzing power and differential cross section for the $10.84 \mathrm{MeV}$ state were found to exhibit deviations from the systematic behaviour seen for the 4.44 and $9.64 \mathrm{MeV}$ states. These deviations are probabiy attributable to the $2 s_{1 / 2}$ transition density components.

2) Iso'vector states of natural parity The $2^{+} T=1$ state at $E_{x}=16.11 \mathrm{MeV}$ The Cohen-Kurath transition density for this state 
is dominated by the $\left(p_{1 / 2} p_{3 / 2}^{-1}\right)$ configuration, like the $1^{+}$il state at $15.12 \mathrm{MeV}$. There is an admixture of the $\left(P_{3 / 2} P_{1 / 2}^{-1}\right)$ configuration, but this $1 \mathrm{~s}$ much weaker. The $s=0$ and $s=y$ ampitrudes in the Cohen-Kurath wavefunctions are of comparable magnitude, and the relative importance of these ampirudes is then determined by the importance of the parts of the force which dominate the interaction. Reiative to this wavefunction, electron scattering data show a. suppression of the iongltudinal and transverse form factors associated with this state 53,55$)$. Consequently the $s=0$ and $s=1$ amplitudes can be renormalized to accomodate this suppression. The normalization factors used in the LS representation 56; were 0.50 and 0.84 for the $S=\emptyset$ and $S=1$ components respectively. The resulting transition density is stili dominated by the $\left(P_{1 / 2} P_{3 / 2}^{-1}\right)$ configuration, but the $\left(P_{3 / 2} P_{1 / 2}^{-1}\right.$ ) term is suppregsed, being replaced by a contribution of opposite phase from the $\left(p_{3 / 2} p_{3 / 2}^{-1}\right)$ configuration.

The excitation of this state can be mediated by the $E_{\tau^{\prime}}^{C} t_{\sigma \tau^{\prime}}^{c} t_{\tau}^{L S}$ and $t_{\tau}^{T}$ components of the interaction. The iso $?^{\prime}$ vector spin-orbit component is generaily very smail and máy be neglected. The dominance of the $s=$ or $s=1$ ainplittudes for the central component of the interaction may be determined by evaiuating the ratio $\left|\frac{t_{\sigma \tau}}{t_{\tau}}\right|^{2}$ as a function $\phi E q$ and $\varepsilon_{p}$. For the $425 \mathrm{MeV}$ force this quantity is greater than 1 and the $s=1$ amplitude is more significant for ali $q$. This is true for lower energies also, as has been pointed 
out by Eomfort et. al.9). For the $550 \mathrm{MeV}$ force, the ratio is unity for $q=0.5 \mathrm{fm}^{-1}$, but for larger $q$ it is iess than unity, and the $s=0$ amplitude is more significant.

The results of the calculations using standard CKWE are presented in Figures VI-20 to VI-22. The renermailized transition densities were used in the computiations presented in Figures VI-23 to VI-25.

The DWIA calculations affirm the dominance of tha real isovector tensor exchange part of the interaction, $t_{\tau}^{T}$, togother with contributions from the real central terms. The spln-orblt terms play almost no role. We discuss flrst the features of the fits using the standard CKWF.

For $E_{p}=398$ MeV the calculated differential cross section is seen to overestimate the data by a factor of 1.85. Similar factors for $E_{p}=597$ and $698 \mathrm{MeV}$ are 1.2 and 1.5 respectively. Rescaled plots are shown by the solid curves in Flguse VI-26. The data for the higher beam energies are well reproduced after rescaling. This is not true for $398 \mathrm{MeV}$ where the calcuiation is seen to poak at $q$ = $1.10 \mathrm{fm}^{-1}$ while the data peak at $q=0.95 \mathrm{fm}^{-1}$. This phase differance resuits in the calculation's over-estimating the data for $q>1 \mathrm{fm}^{-1}$ even after scaling. For this particular set of transition densities it is elear that the dominant effect of the 1sovector tensor part of the force is decreasing for increasing beam energles. The enhancement due to 
$E_{x}=16.11 \mathrm{MeV} 2_{1}^{+}: 1-$ Differential Cross Section Calculations (Unquenched)
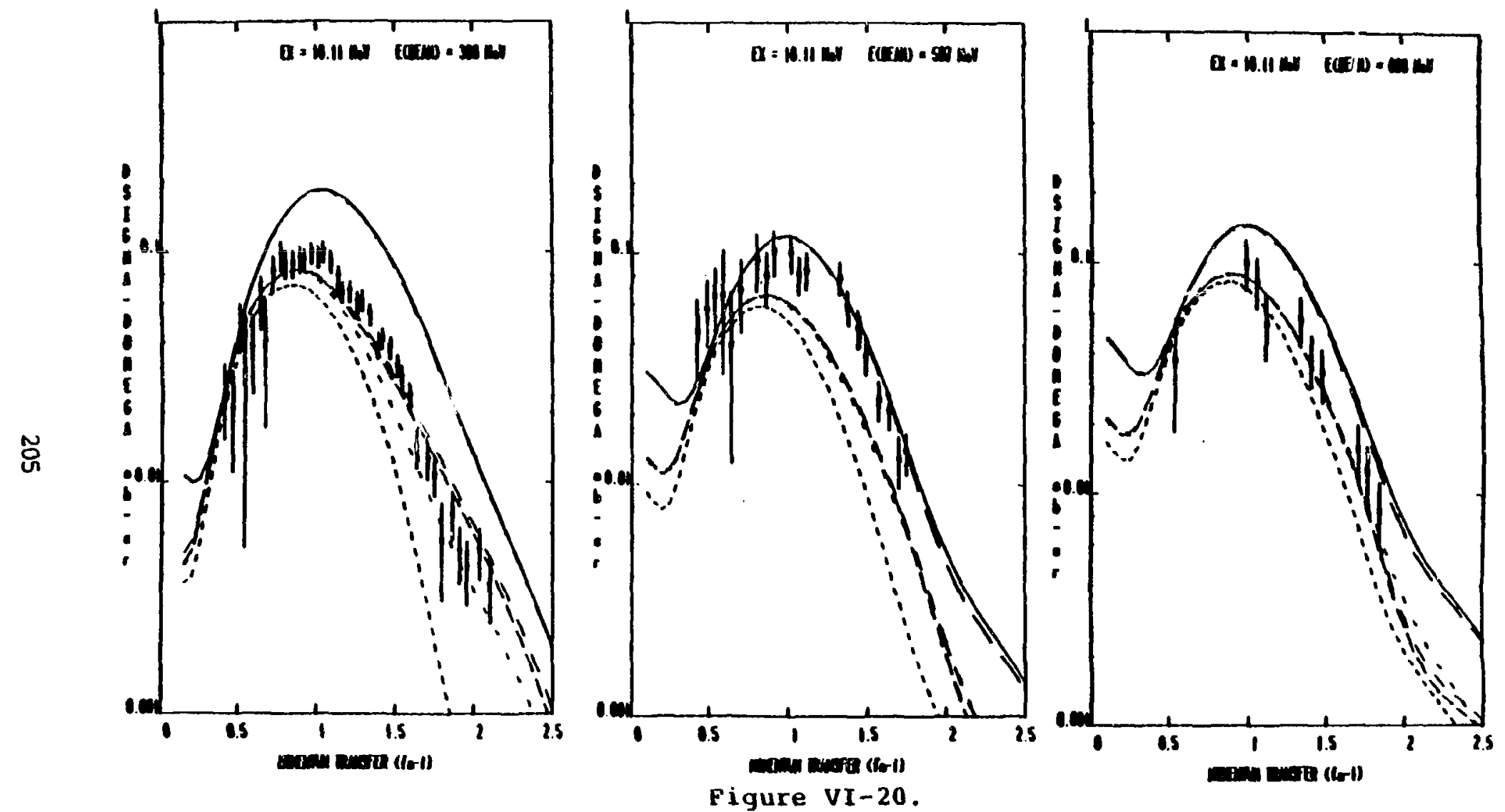
$E_{\mathbf{X}}=16.11 \mathrm{Mev} 2_{1}^{+} ; 1$ - Analyzing Power Calculations
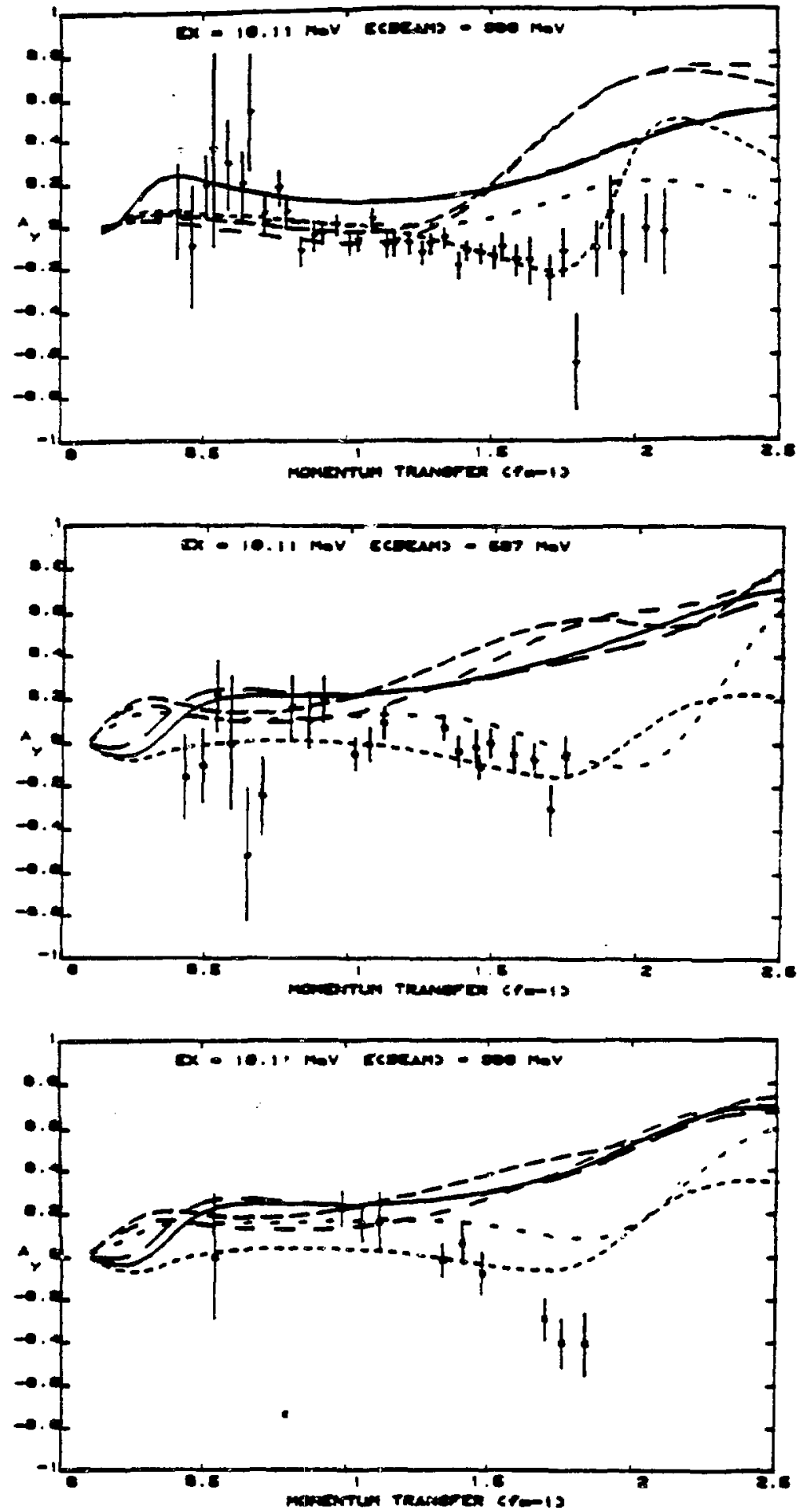

Eigure VI-21. 
$E_{x}=16.11 \mathrm{MeV} 2_{1}^{+} ; 1$ - Differential Cross Sction Calculations (Quenched)
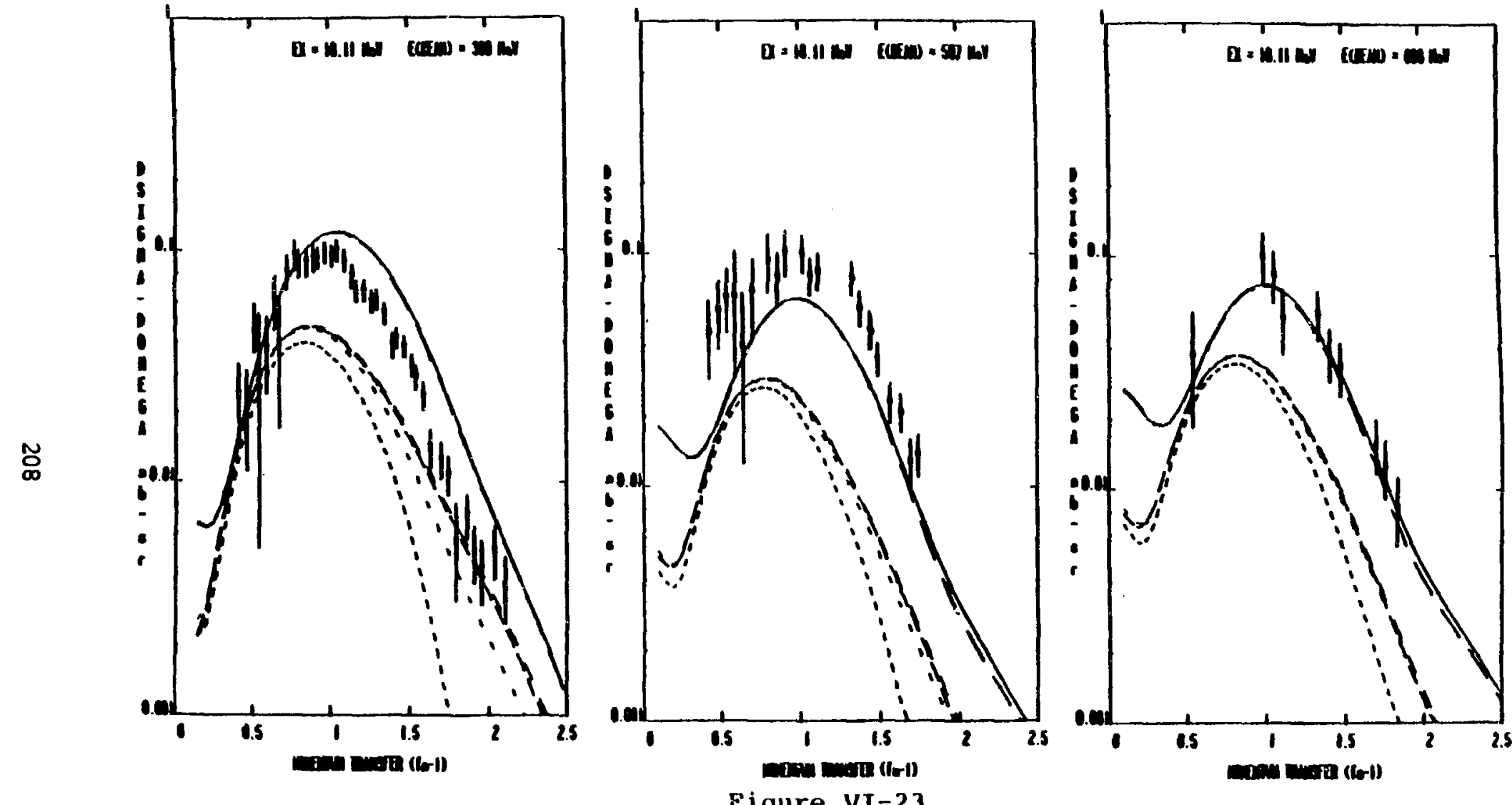

Figure VI-23. 

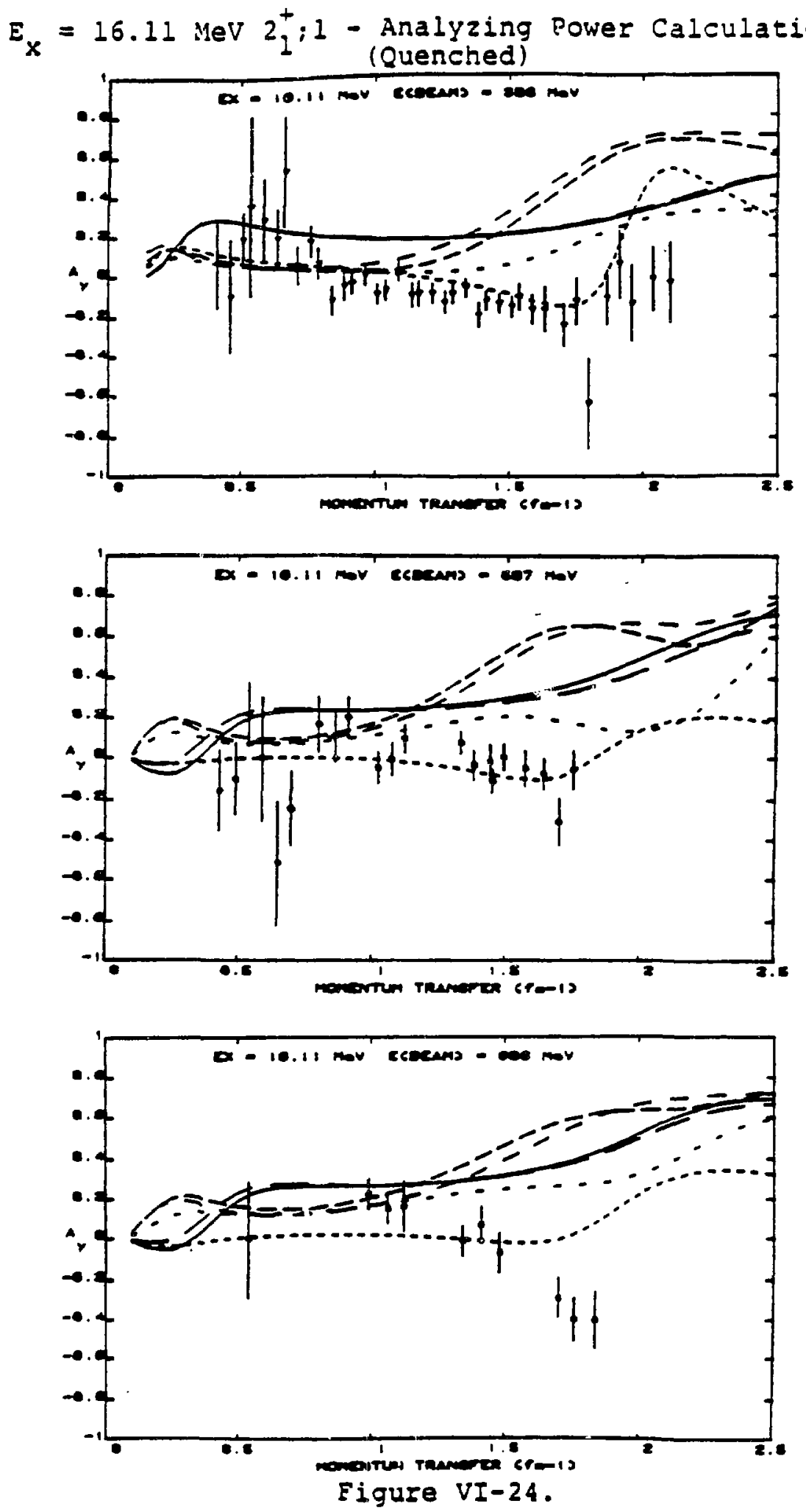
$E_{x}=16.11 \mathrm{MeV} \mathrm{2} 2_{1}^{+} 1$ - Comparative Calculations for Observables (Quenched)
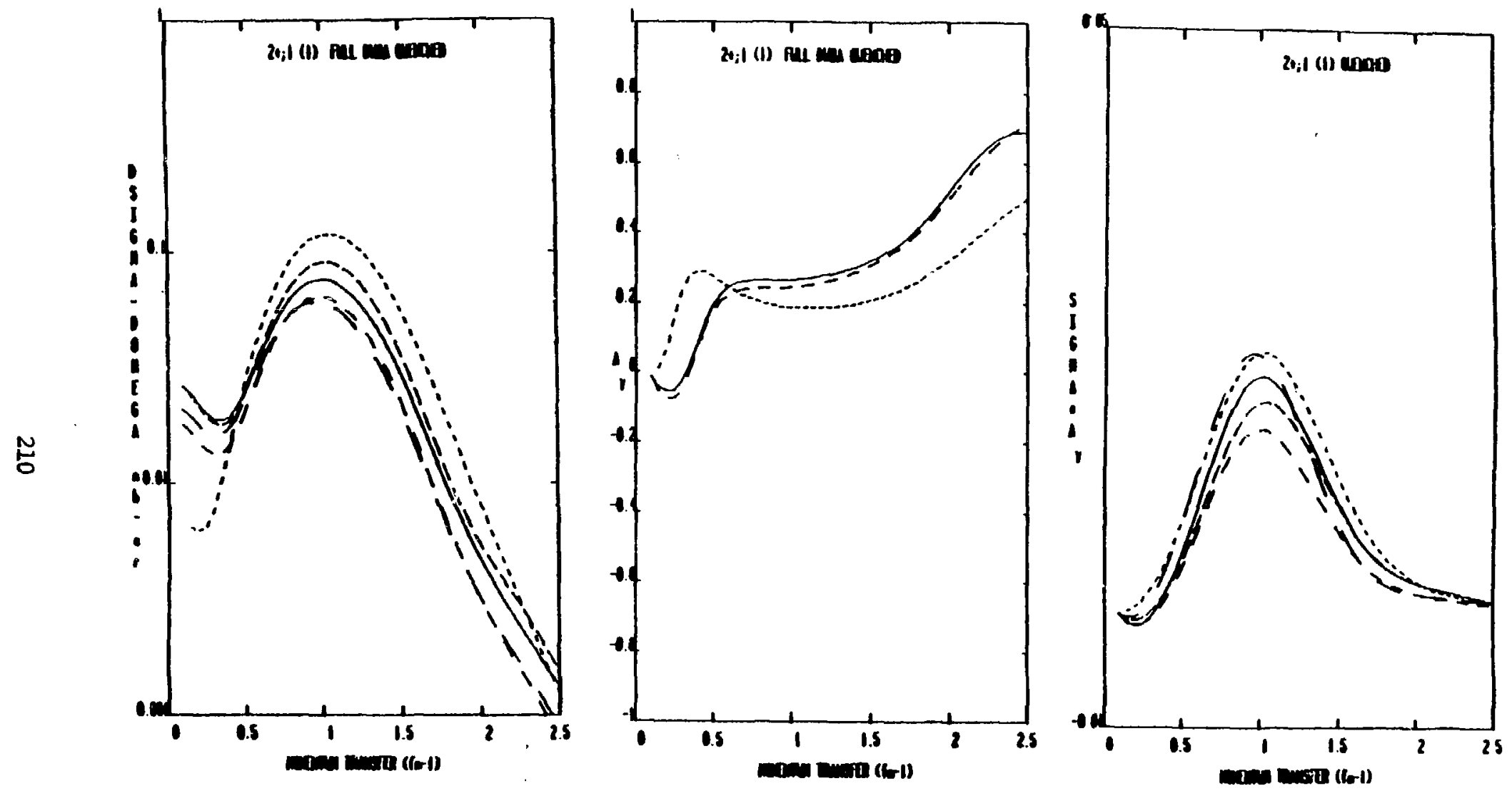

Figure VI-25. 

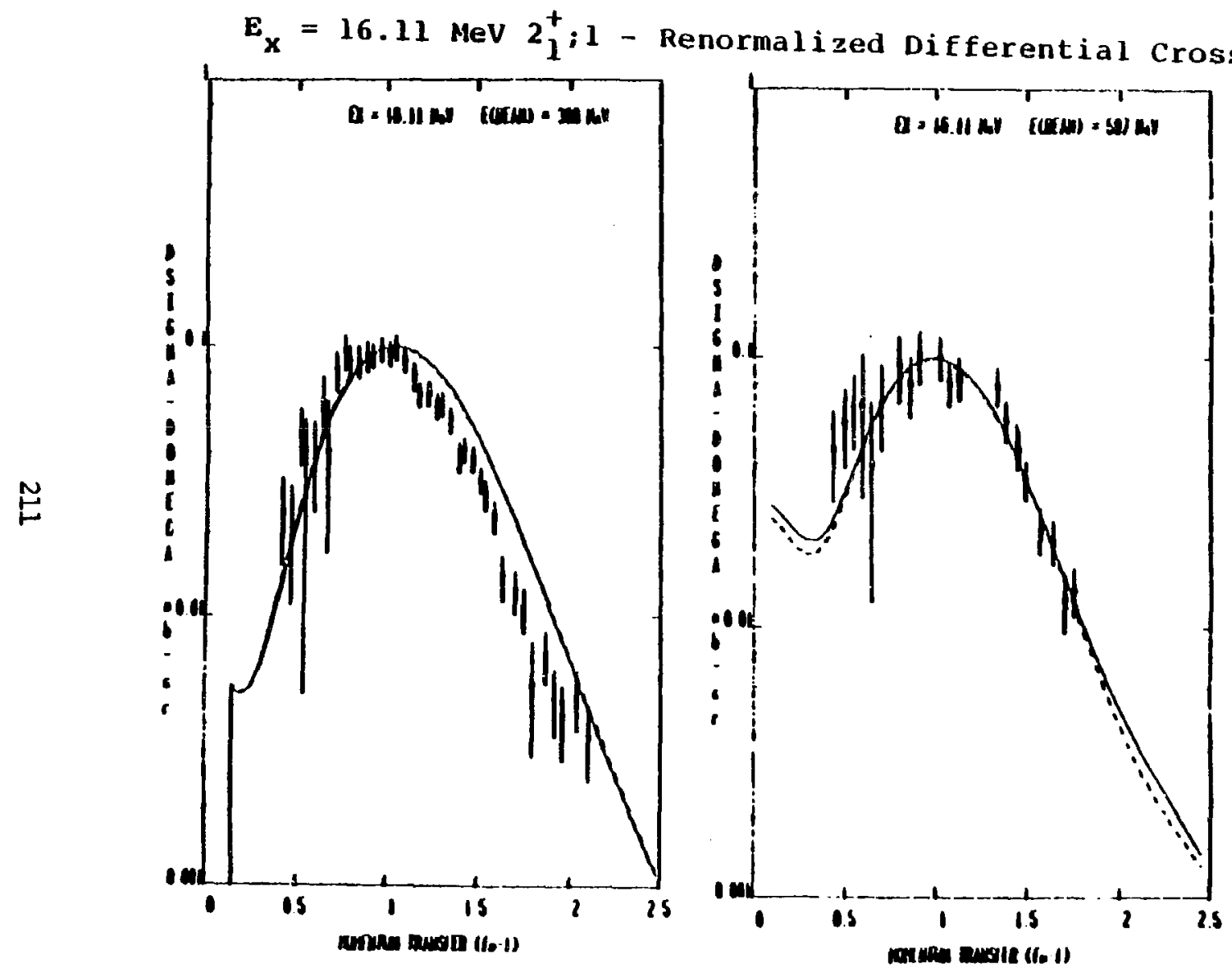

Figure VI-26.

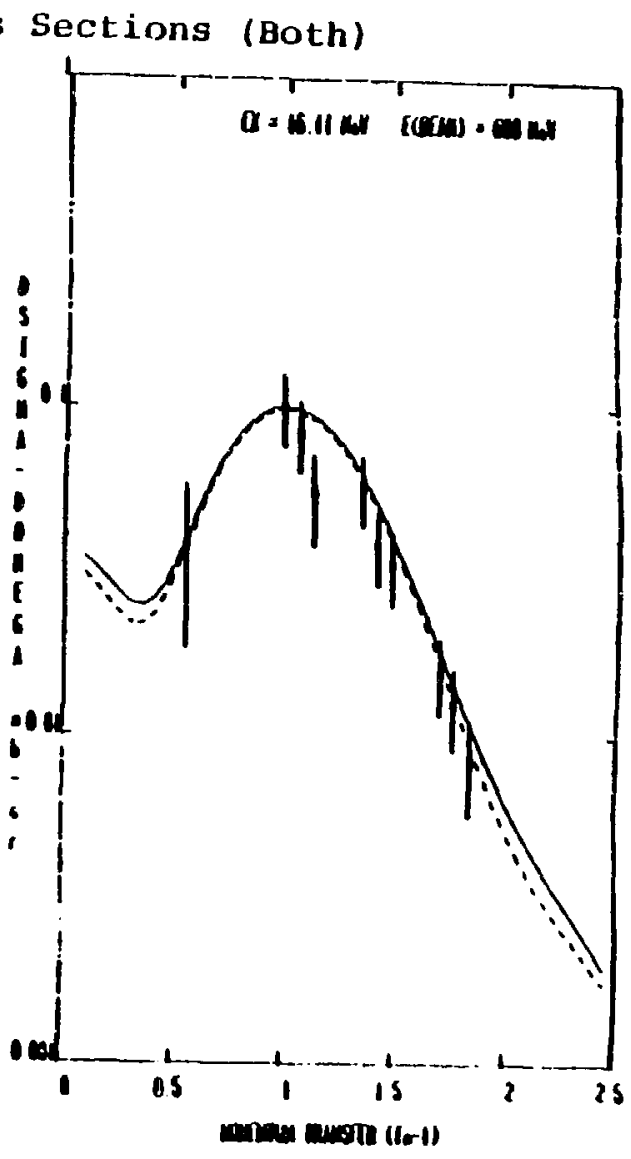


this component at $q=1.5 \mathrm{fm}^{-1}$ is reduced from 3.3 at 398 $\mathrm{MeV}$ to 2.3 at $597 \mathrm{MeV}$ to 2.1 at $698 \mathrm{MeV}$. Aiso, the centrai term shows a decrease of some 30 from $398 \mathrm{MeV}$ to $597 \mathrm{MeV}$, then rising again to a value at the maximum for 698 MeV which $1 \mathrm{~s}$ comparable to that for $398 \mathrm{MeV}$. The jow momentum transfer behaviour aiso changes signifleantiy from 398 MeV to $597 \mathrm{MeV}$. The cross section for the smallest vaiues of $q$ is enhanced relative to that for $398 \mathrm{MeV}$ by a factor of three for $597 \mathrm{MeV}$ and five for $698 \mathrm{MeV}$. Aiso, a pronounced minimum is seen at $q=0.4 \mathrm{fm}^{-1}$ for 597 and $698 \mathrm{MeV}$. Such a minimum is absent in the $398 \mathrm{MeV}$ calculation, as well as in the Iower energy calculations of Comfort et. ai. ${ }^{91}$. Examtnation of the $q$ and $E_{p}$ dependence of $t_{\tau}^{T}$ as determined by Love and Franey shows the shape of the low momentum transfer region to be largely determined by this part of the force.

Renormalization of the transtion densities results in oniy minor differences in the overali shape of the calcuiated cross sections, as is seen from the dashed ines in Figure VI-26. Some small differences for $q \leq 0.5 \mathrm{fm}^{-1}$ and $q$ $\geq 1.5 \mathrm{fm}^{-1}$ are seen; the renormalized transition densities yielded slightly lower values than the standard CXWF. Renormalization provides a very good fit to the data for $E_{p}$. $698 \mathrm{MeV}$, an improved fit to the data for $E_{p}=398 \mathrm{MeV}$. and a poorer fit to the data for $E_{p}=597 \mathrm{MeV}$. The calculation continues to overestimate the 398 MeV data, but now only by a fector of 1.2. This renormalization factor is consistent 
with those noted by comfort et. aj.9) and Haji-saied et. al. 15$)$ and with that required for electron scattering ${ }^{53}$. The 597 MeV data are now underestimated by a factor of 1.6 .

The enhancement factors for the $t_{\tau}^{\mathbf{T}}$ term at $q=1.5$ $\mathrm{fm}^{-1}$ are $4.0,3.7$ and 3.2 for the energies 398,597 and 698 MeV respectively. The central term retalns the same behaviour as seen without transition density renormailzation. At the smaliest values of $q$, the $t_{\tau}^{T}$ term contributes an enhancement in the cross section relative to that at 398 MeV of a factor of 2.8 for $597 \mathrm{MeV}$ and 4.3 for $698 \mathrm{MeV}$. It is also evident that the contribution of $t_{\tau}^{T}$ to the cross section for sinail $q$ is rore significant by a factor of two than for the standard CKWE.

In summary, the renormalization of the transition censities results in an overali reduction in the strength of the cross section. Eurthermore, the contribution from the isovector tensor term is enhanced for al beam energies, particulariy for the $650 \mathrm{MaV}$ force.

The Elts to the analyzing power data are poor at Iarge momentum transfer. No significant difference is observed between standard and renormalized transition density calculations for $A_{Y}$. Whereas the measured anaiyzing powers become negative fot $g>1.4 \mathrm{fm}^{-1}$, the calculations give monotonicaliy increasing positive values from $\mathrm{g}>\mathrm{I}_{\mathrm{fm}}^{-1}$. Some modicum of agreement is seen for small g, but calcula- 
Elon and data cleariy diverge for large $q$. The source of the divergence is not readily obvious because of complicated interferences which oceur between contributions from various parts of the force.

Analyzing power data for this transition have been reported by comfort et. aI. Eor energies of $122^{7}$ ) and 2009) Mev. The dato at these energies are different in structure from the data reported here, being in general positive but showing some strueture for iarge momentum transfer (2-2.5 $\mathrm{fm}^{-1}$ ). DWIA caiculations at these energies give resuits not compieteiy consistent with the data, but quite dissimilar to those reported here, the Ay becoming negative in the large 9 region.

It is remarkabie that this state should show a very small dependence on incident beam energy as compared with the $2_{l}^{+}: 0$ state. The calculations presented here, together with those of Comfort and gaj1-saled, show a slight decrease in magnitude as incident energy increases, consistent with the data. It should also be pointed out that the data at $398 \mathrm{MeV}$ ace conststent with those of Escudle et. al. at 402 Mev. The dominance of the isovector tensor term makes it difficult to examine the $q$ and $E_{p}$ dependence of the central isovector terms, but the central contribution to the fuli cross section is roughly consistent with the dependences predicted by Love and Franay. 
The Eits to the cross section data out to about 2 $\mathrm{Em}^{-1}$ suggests that the lsovector tensor and centrai components of the interaction, and their energy dependence, appear to be correct over this range of momentum transfer to within a factor of about 1.2. Furthermore, the renormalization of the calculation based on electron scattering data appears to be correct.

The serious difficulties with the analyzing power caicuiations are not easily understood. Knock-on exchange terms are found to reduce the cross sections as has been noted for isoscaiar natural parity states, but the effect is somewhat reduced from a factor of 3.5 to about 2 .

3) Isoscaiar states of unnatural parity

The $1^{+} T=0$ state at $E_{X}=12.71 \mathrm{MeV}$

The dominant transition densities associated with this state are $\left(p_{1 / 2} p_{3 / 2}^{-1}\right)$ and $\left(p_{3 / 2} p_{1 / 2}^{-1}\right)$, the former being twice as strong as the latter. Smali contributions from the reartangement configurations within the $p_{1 / 2}$ and $p_{3 / 2}$ subshells are also present, and these were included in the calculations. The harmonic osciliator parameter used in the computations was $1.572 \mathrm{fm}$.

The comparison of the results of the DWIA caiculations with the experimental data are presented in Figures 
$\because \mp-27$ and VI-28. The fuil curves for a variety of forces at different energles are presented in figure VI-29.

An excelient fit to the differential cross section data for $q$ up to $1.4 \mathrm{fm}^{-1}$ is obtained for $E_{p}=398 \mathrm{MeV}$. For q $>1.4 \mathrm{fm}^{-1}$, the fuil caiculation is seen to underestimate the data, and alse to show less structure than is evident in the data. We note the constancy of the cross section projected back to $q=0$ at this beam energy. The fit to the cross section data for $\varepsilon_{p}=597 \mathrm{MeV}$ is poor. A serlous discrepancy occurs for smaid vaiues of $q$ where the calculated curve underestimates the measured cross section, and fails off as q goes to zero, contrary to what is observed for $E_{p}=398 \mathrm{MeV}$. For $0.8 \mathrm{fin}^{-1}<q<1.2 \mathrm{fat}^{-1}$ agreement with the data is reasonabie, but as for the 398 MeV data the caiculation underestimates the data for $q>1.2 \mathrm{fm}^{-1}$. A more pronounced structure in the caiculation for the $597 \mathrm{MeV}$ case is observed in the 1.6 to $1.8 \mathrm{fm}^{-1}$ region of $q$, which appears to better reproduce the shape of the data in this region. The fit to the cross section data for $E_{p}=698 \mathrm{MeV}$ is seen to exhlbit some of the same characteristics as that for the $597 \mathrm{MeV}$ data. Agreement for smail $q$ is poor, the calcuiation also falling off as g tends to zero. Agreement in the intermediate range of $q$ is good, and while the data for $q>1.4 \mathrm{fm}^{-1}$ are again underestimated, agreement is much closer than for the lower energies. The shape in the iarge momentum bansfer region is reasonabiy reproduced, partiy 

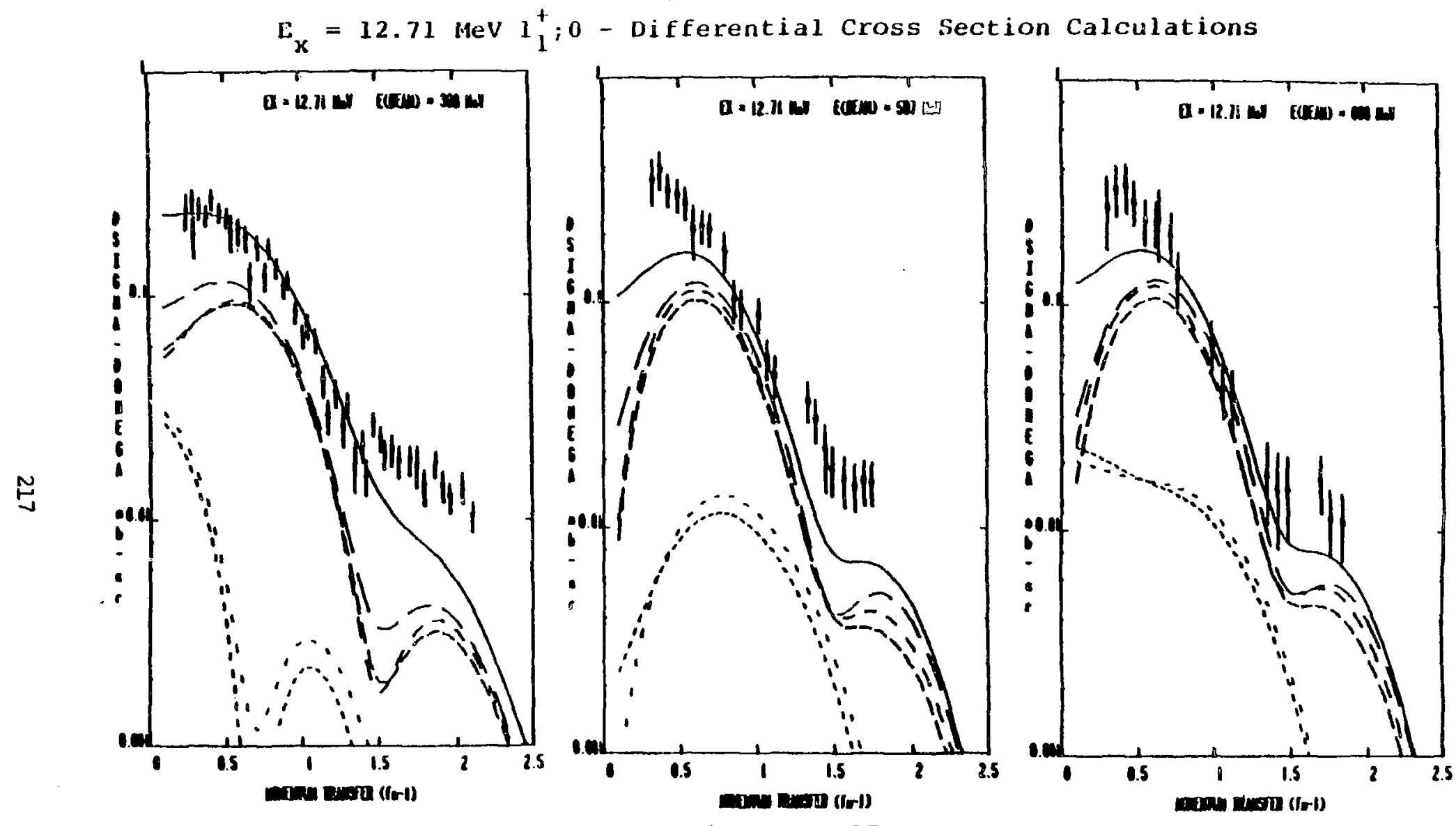

Figure VI-27. 

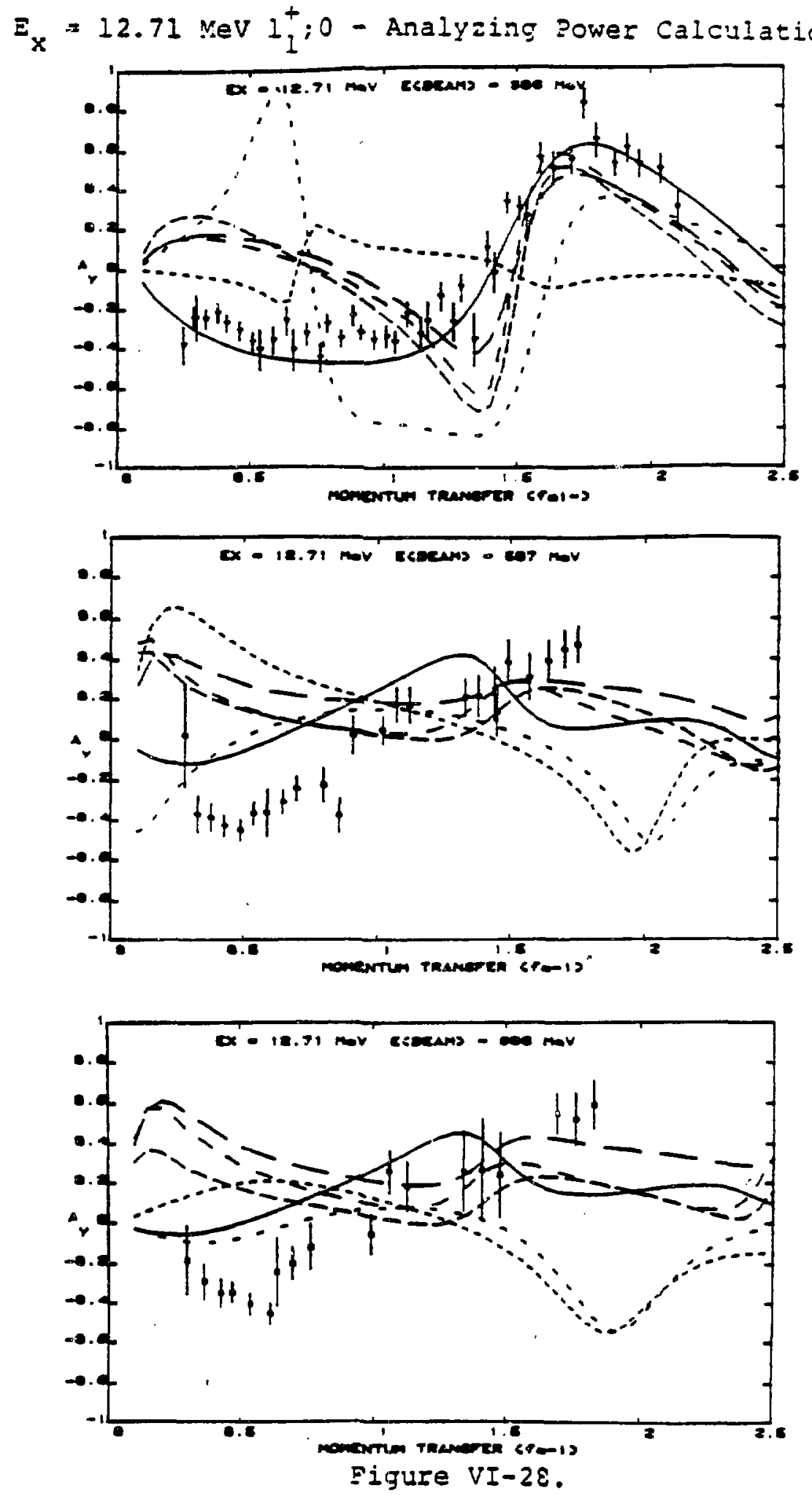
$\mathrm{E}_{\mathrm{x}}=12.71 \mathrm{MeV} \mathrm{1}_{2}^{+} ; 0$ - Comparative Calculations for Observables
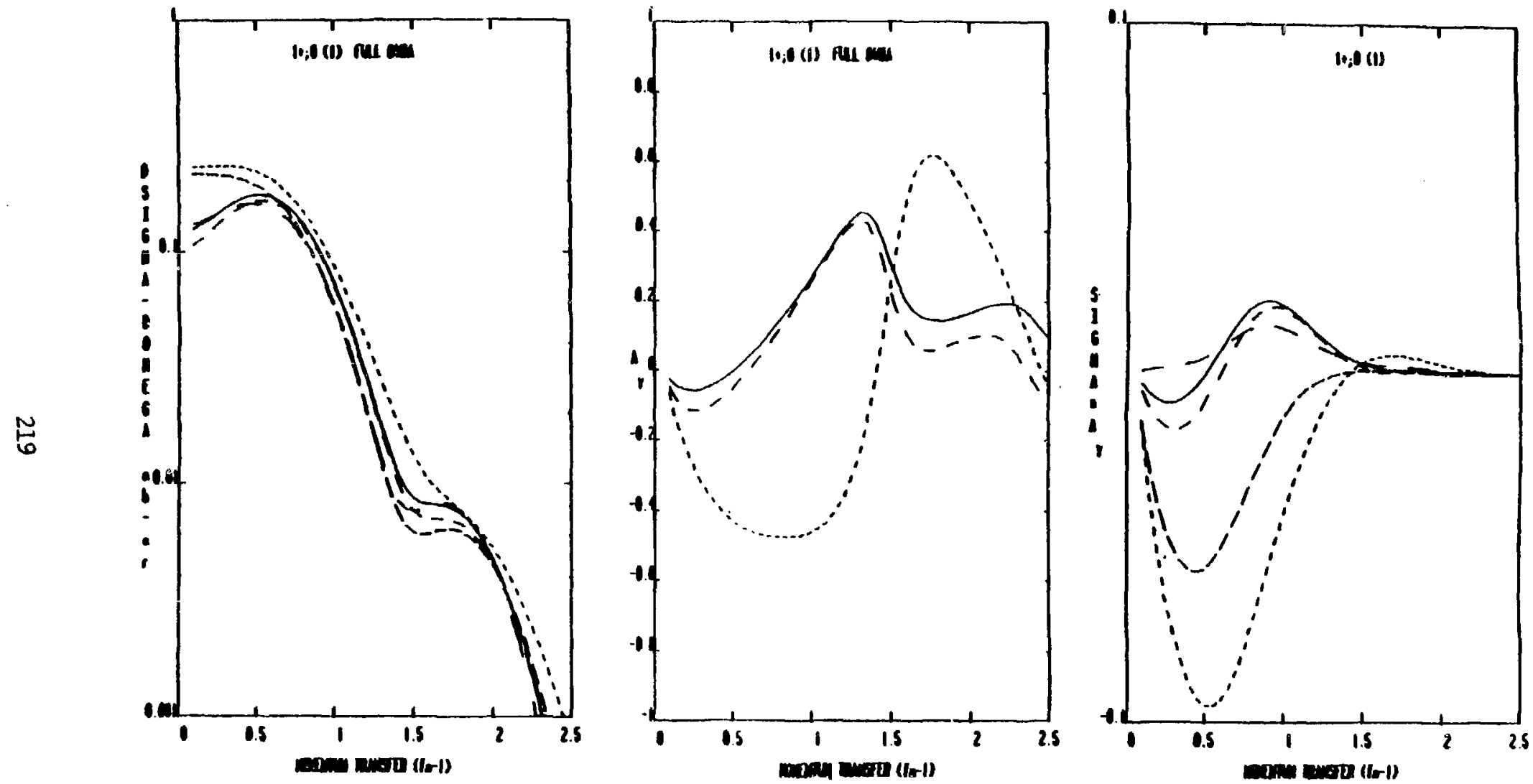

Figure VI-29. 
vecause error bars are iarge.

Comparison of the calculations with the analyzing power data show. a remartable degree of surcess for $E_{p}=398$ MeV at all momentum transfers. Unfortunately, agreement for the higher beam energies is not as good. The higher energy calculations do not exhiblt the same distinctive negative values for low $q$ seen in the data, and a clear difference in phase between calculations and data is apparent. The phase difference between the calculations for the three different beam energies is most clearly evident in Figure VI-29.

The caicuiations show that the dominant wechanim for the excitation of this state is the imaginary tensor exchange part of the force, especially at iow q and $398 \mathrm{MeV}$. The spin-filp part of the central force is weak, while the reai isoscaiar spin-orblt component plays a significant role. of interest is the change in shape for low $q$ of the caiculated cross section in going from the $425 \mathrm{MeV}$ force to the 650 MeV force, as weIl as the marked difference in shape for $A_{y}$ yleided by these two descriptions of the force. The tensor exchange contribution is decreasing as a function of energy in the intermediate momentum transfer reglon, but is roughly constant for the smallest yalues of $q$, and does not explain the observed dffferences. It appears that a reduction in the spin-orblt strength in the low q region resuits in the reduction of the forward angle calculated cross section for the $650 \mathrm{MeV}$ force. Since both the 597 and $698 \mathrm{MeV}$ 
caiculations were done with the 650 Mev force, it is reasonabie to suspect a deflciency in the force at this energy to explain the observed behaviour.

To further investigate this probiem, computations were performed using the $597 \mathrm{MeV}$ optical potentlals with the $515 \mathrm{MeV}$ force to attempt to reproduce the $597 \mathrm{MeV}$ data, and using the $698 \mathrm{MeV}$ optical potentials and the 800 MeV force to attempt to reproduce the $698 \mathrm{MeV}$ data. The $800 \mathrm{MeV}$ force showed the same behaviour as the $650 \mathrm{MeV}$ force, and produced no signjficant improvement. The 515 MeV force, however, generated a smaij momentum transfer dependerice in the observables which fit the data better, and aiso were more consistent with the results at $398 \mathrm{MeV}$. This Iorce has about the same spin-orblt strength as the $425 \mathrm{MeV}$ force, but less tensor strength.

We recail that the $597 \mathrm{MeV}$ optical potential was somewhat suspect. Several caicuiations were performed to deduce the sensitivity of the low momentum transfer dependence of the calculations to various terms in the optical potential. Removing either the real central of surface imaginary terms resulted in no signiflcant change in the cross sections. The calculated analyzing powers were also insensitive to these changes. The effects of removing the spinorbit distortions from the entrance and exit channeis at both 597 and 698 Mev are minimal. A small change in the overald slope of the cross section is obseryed, and the 
anaizying powers showed little change.

This Insensitivity of the calcuiated observables to the opeical parameters and spin-orbit distortions indicates that the poor fits to the data, especially at low q, are an artifact of the force rather than adjustable parameters. The difficulty seems to ils with the real isovector component of the spin-orblt force, espectaliy for $q<0.5 \mathrm{fm}^{-1}$. The fact that this discrepancy oceurs for both the 597 and $698 \mathrm{MeV}$ data when both are described by the $650 \mathrm{MeV}$ force is also an indication. This is more obvious when the $515 \mathrm{MeV}$ force removes a substantial part of the discrepancy when used to describe the $597 \mathrm{MeV}$ data. The weak nogative analyzing power for low $q$ calculated from the 650 MeV forse is a further indication of a deficiency in the description of the spin-orblt force at this energy.

Extensive studies of this transition have aiso been made by several authors at a variety of incident proton energies, as well as with other probes. Escudie et. al. measured the diffarential cross section for this trangition at $E_{p}=402 \mathrm{MeV}$, and while forward angle agreement with the data presented here is reasonable, the $402 \mathrm{MeV}$ data drop glightly more rapidly for $\mathrm{q}>1.5 \mathrm{fm}^{-1}$ than the $398 \mathrm{MeV}$ data. The source of this difference remains unclear. Comfort et. ai. have reported differential cross section data for $E_{p}=122,185$ and $200 \mathrm{MeV}$, and analyzing power data for $E_{p}=122$ and 200 Mev5-7,9). haji-Saled et. al have report- 
ed data for $\Sigma_{p}=800$ MeV togetier with DWIA caicuiations ${ }^{15)}$. What is striking about these daca is the significan degree of Insenstefvity of both the magnitude and shape of the cross section as a function of incident particie energy. There is some energy dependence for the smailest momentum trarisfers, the cross section risting gradualiy in this region as the incident energy rises. The shoulder at $q=1.4 \mathrm{fm}^{-1}$ is ciearly evident in all the data, and appears to retain its shape over the entire energy range.

Agreement with calculations, though, exhibits a definite energy dependence. Fits to the differential cross sections for $E_{p} \leq 200$ MeV badiy overestimate the data for the smaliest momentum transfers, while underestimating at iarger momentum transfers $5-7,9)$. At 800 MeV there is a sight underestimation of the data at smaijest angles, agreement at intermediate angies and underestimation for large angies ${ }^{(5)}$. The calcuiated cross sections are aimost Invarlant as a function of energy with the exception of those involving the $650 \mathrm{MeV}$ force and $597 \mathrm{MeV}$ opticai potential. The slight energy dependence of the data for low $q$ consequentiy results in better fits to the data in the 400 to $800 \mathrm{MeV}$ range; at large q the data and the calculations are about the same as a function of energy.

Electron scattering is not of much assistance in understanding the behavlour of this state. There is a large degree of interference and consequent canceliation between 
spin and orbitai contributions, and aiso high sensitivity to isovector admixtures. While smali isospin admixing has been proposed 57,58$)$, the eftects on $\left(p, p^{\prime}\right)$ scattering are negligibie. The Cohen-kurath wave functions are found to underestimate the measured transverse form-factors by about a Eactor of four 531 .

The analyzing powers reported by comfort et. al. are in marked disagreement with calcuiations at $122 \mathrm{Mev}^{7}$ ) while agreement is much improved at $200 \mathrm{MeV}^{9}$ ). The calculations yield strongly negative values for low q, crossing to positive values in the $1 \mathrm{fm}^{-1}$ region as we have seen for the caiculations performed in this work. The data at $122 \mathrm{MeV}$ are not negative for Iow $q$, but this disagreement is an exception to the systematies observed from $200 \mathrm{MeV}$ to $700 \mathrm{MeV}$.

As has been mentioned, the knock-on exchange terms are essential for the description of the tensor term. Interference between direct and exchange amplitudes resuits in a reduction in the differential cross section by a factor of 2 from the direct calculation only.

4) Isovector states of unnatural parity.

(a) The $1^{+} T=1$ state at $E_{X}=15.11 \mathrm{MeV}$

The dominant transition densities are $\left(p_{1 / 2} p_{3 / 2}^{-1}\right)$ and $\left(P_{3 / 2} P_{1 / 2}^{-1}\right)$, the ratio of the sirengths being $2: 1$. Smali contributions from rearrangement conflgurations within the 
$P_{1 / 2}$ and $P_{3 / 2}$ subsheis are also present, and were included in the calculations. The harmonic osciliator parameter used for this state was $1.866 \mathrm{fm}$, the Iargest for any state and required to flt the electron scattering form Eactor ${ }^{54}$ ).

This transition is primarily mediated at small q by the central spin-isospin filp part of the nucleon-nueleon amplitude, which is dominated by one pion exchange. The tensur isovector term $t^{T}$ plays a dominant role for larger momentum transfers. The Cohen-kurath transition densities used in the computations are belisved to be rellable for $q \leq$ $1 \mathrm{fm}^{-1}$, but not for values of $q$ iarger than this 53,54$)$. The Cohen-kurath wave functions are successfui in reproducing the shape of the inelastic eiectron scattering transverse form- factor out to about $1.5 \mathrm{fm}^{-1}$, but fali to reproduce a second maximum near $q=2 \mathrm{fm}^{-1}$. The quaitity of the fit to the data for $q \leq 1.0 \mathrm{fm}^{-1}$ wouid then furndsh information concerning the vaildity of the force, assuming the transition density in this region to be well-defined.

Comparison of the calculations with the data are presented in Figures VI-30 and VI-3I, and the calculations are compared with each other in Figure VI-32.

The DWIA calculations show the forward angle cross section to be dominated by the reai somponent of $t_{\sigma \tau}^{c}$, while the real isovector tensor term plays an important role in enhancing the cross section for $q>0.5 \mathrm{fm}^{-1}$. The spin- 
$E_{x}=15.11 \mathrm{MeV} 1_{1}^{+} ; 1$ - Differential Cross Section Calculations
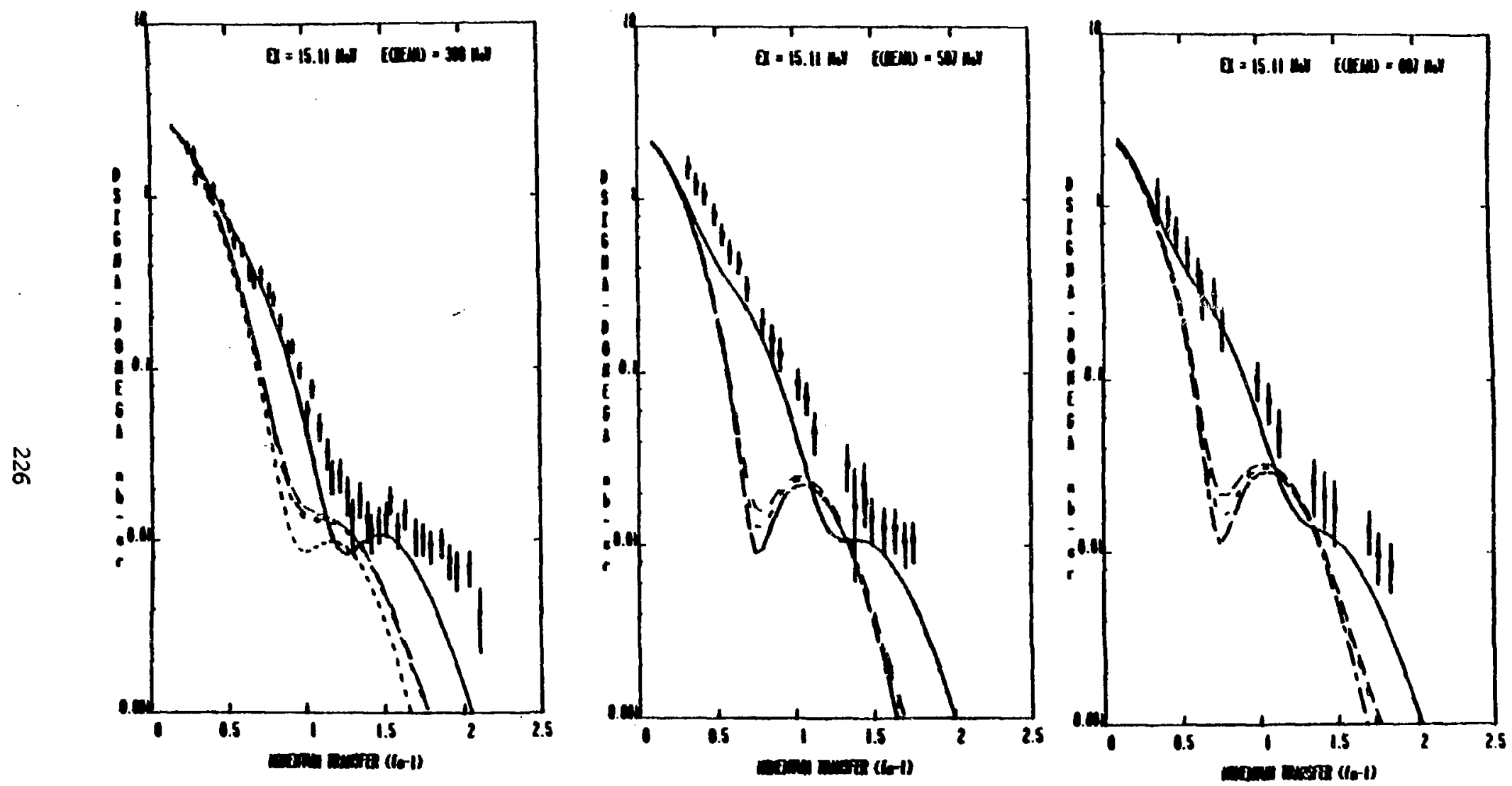

Figure VI-30. 


$$
E_{x}=15.11 \mathrm{MeV} I_{1}^{+} ; 1 \text { - Analyzing Power Calculations }
$$
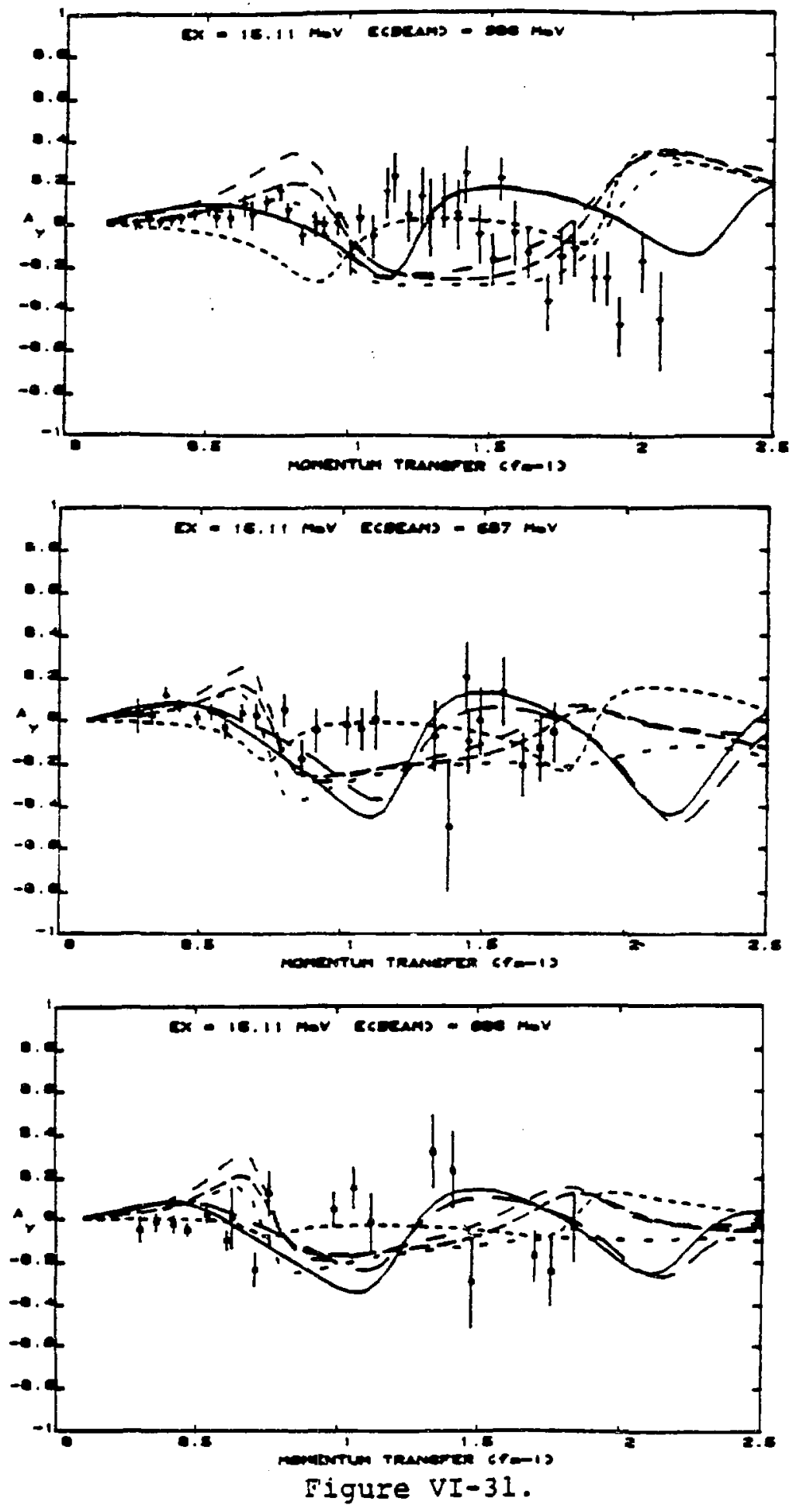

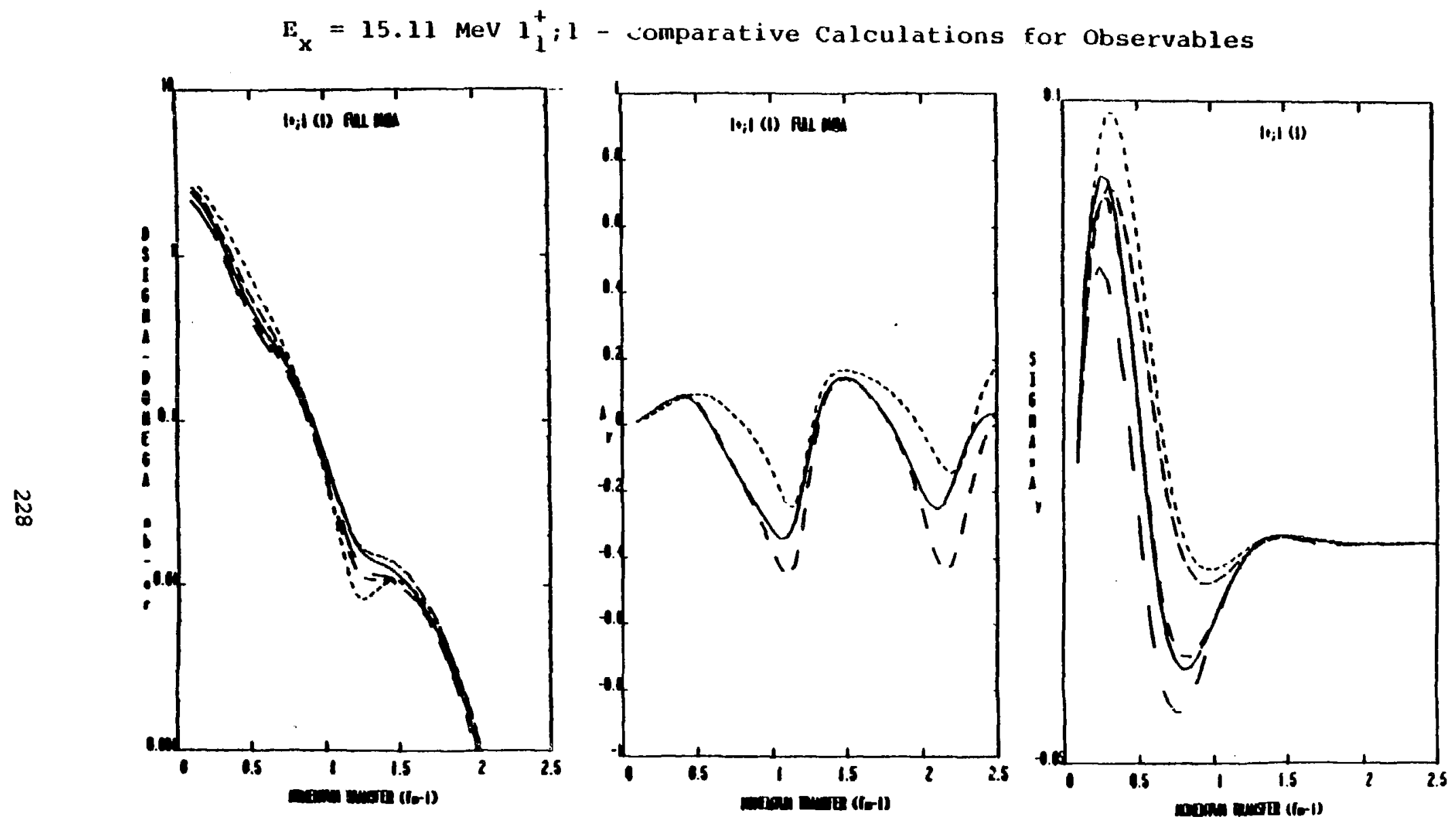

Figure VI-32. 
orbit components are seen to have an aimos: negiigibie efsect. The fit to the cross section din for $E_{p}=398 \mathrm{MeV}$ up to $q-0.6 \mathrm{fm}^{-1}$ is exceijent. Adspement is reasonabie for $E_{p}=698 \mathrm{MeV}$ over the same region of $q$, while the fit to the data at $E_{p}=597 \mathrm{MeV} 1 \mathrm{~s}$ poor in tisis range. The calcuiation for this iatter beam energy underestimates the data by factor of abous 1.6 .

The calculations for the analyzing powers show a consistency in shape and phase as a function of lncident particle energy. This is in contrast to the more significant energy dependence seen for the isoscalar $1^{+}$state discussed previously. The analyzing power for $q>0.5 \mathrm{fm}^{-1}$ appears to be strongiy infiuenced by the tensor exchange component of the interaction. In general the smail g data are reasonabiy fit by the calculation. The shapes of the data and the calcuiations are consistent, but a definite phase difference between data and caiculation at each beam energy is seen, although the relative phases of the calculations are constant. The first minimum in the caiculated anaiyzing power occurg at a larger value of $q$ than in the data, becoming progressively worse as the bean energy increases. The location of the minimum is colnctdent with the location of the dip in the calculated cross section.

Thls transition, because of the reliability of the Cohen-kurath wave functions for low $q$, provides a good test of the components of the effective interaction in this re- 
gion. Since we find exceilent agreement for the iow momentum transfer region at $398 \mathrm{MeV}$ and reasonabie agreement at 697 MeV, the spin-isospin Elip component of the interaction describing this transition appear to be weil-defined. The discrepency at $597 \mathrm{MeV}$ may to some extent be removed by use of the 515 MeV parameterization which gives a sightiy larger cross section than the $650 \mathrm{MeV}$ force at this energy.

This particular state has been the subject of much recent interest in the search for evidence of precritical phenomena related to pion condensation in the nucleus. CaIcuiations using the Landau Eermi-liquid theory by Tok 1 and Weise and others predict a significant peak (about a factor of three) in the differential cross section for $q-1.6-2.0$ $f^{-12(b-23)}$. No evidence for such an enhancement is seen in the data, and this, together with the fact that the shape of the data is reasonably reproduced by a standard DWIA calcuiation, ailow us to conciude that such evidence for precittcal phenomena is not seen for this state at these beam energies. The discrapancies seen here between theory and experiment are comparable to those observed for other states. specificaliy the 12.71 MeV $1^{+}$; 0 state Eor whlch no precritical phenomera should exist. This conciuston is compatible with similar observations made by comfort et. aI $6,9,52)$ and Hajt-saeld et. al. ${ }^{14}$ ) at lower and higher beam energies.

The disagreements botween calculation and experiment in the high momentum region have proved to be rather in- 
tractabie. Comfort 52 has studied this transition at lower energles in great detail, and conciuded that reasonable variations of available parameters could not account for the observed discrepancies. Similar difficulties have been noted at $800 \mathrm{MeV}$ by Haji-saied et. aj. ${ }^{14)}$. Comfort has nored that for lower energies removal of the [LSJ] = [111] ampl1tude from the transition density, or alternatively the use of the empifical ampiftudes of Dubach and Haxton 59), resulted in some lmprovements. Thts constant problem, though, does not yet have a satisfactory explanation. The calculatlons presented here do not, unfortunately, ald in the resoiution of this difficulty.

The analyzing powers at lower energles show much more structure than $1 \mathrm{~s}$ seen in the results of this work. The caiculations at $122 \mathrm{MeV}$ reasonably reproduce the forward angie data, but exhiblt much the same phase difficulties for iarger angies as seen in this work. The situation at 200 MeV is much worse, the calculation and data being compietely out of phase. This situation may be remedied to a certain extent by the removal of the [111] ampititude.

The effects of spin-orbit distortions on this transition are rather interesting. While having ilttie effect on the low momentum transfer cross section and analyzing power, removal of the spin-orblt distortions causes a significant deepening of the mintmum in the region of the shoulder in the differential cross section at $q=1.2 \mathrm{fm}^{-1}$. Also, 
the first minimum in the analyzing power, corresponding to the cross section minimum, is much sharper in the absence of such distortione. The distortions are thus essential to smooth out the caicuiation in this region, thus bringing the resuits into retter gualitative agreement with the data.

Over the momentum transfer region encompassing the data, the knock-on exchange terms are seen to reduce the direct differential cross sections by an almost uniform factor of two. While such a reduction is generally favourable for low momentun transfers, it possibiy contributes to deficlencles at higher values of $q$.

We finaliy note the remarkable independence of the calculated differential cross sections as a function of ineldent particle energy. Although small deviations are seen for low $q$ and also in the region of the shouider, it is ciear that the $t_{\sigma}^{c}$ and $t_{T}^{T}$ terms which mediate the excitation of this state shouid be approximately constant as a function of $E_{p}$ for different values of $q$. The figures presented in Chapter II attest to the validity of this observation.

(b) The $2^{-} T=1$ state at $\varepsilon_{X}=16.58 \mathrm{MeV}$

The transition density for this excltation is doninated by the $\left(2 s_{1 / 2} 1 p_{3 / 2}^{-1}\right)$ configuration with a significant contribution from the $\left(d_{5} / 2 P^{-\frac{1}{3}} / 2\right)$ configuration. Several other configurations are present but are negligioly small. The osciliacor parameter used in the computations was 1.681 
En, which is the same as used by comfort et. aj.6,9) but iarger than that of 1.55 used by taji-saeid et. al. ${ }^{15}$ ).

The comparisons of the caicuiations with data are presented in Figures VI-33, 34 and 36 , while the caicuiations for varlous energies are presented together in Figure $V I-35$

We note that the differential cross sections, while being reasonably well-réproduced in shape are badly overestimated by the calculations. Renormalization factors of $3.33,0.50$ and 0.57 are required for 398,597 and $698 \mathrm{MeV}$ respectively to generate the Eits is Elgure VI-36. Comfort et. ai. report factors of 0.2 and 0.25 needed to renormalize the caiculations at 122 and $280 \mathrm{MeV}^{6,9)}$. Hajt-saeld et. al. require no scaling to Eit the data at $800 \mathrm{MeV}^{15}$ ), and so the trend is ciear. The measured $z=0 s s$ sections ace aimost independent of energy, while the caicuiated cross sections are monotonicaijy decreasing as the beam energy increases, E1naijy converging with the data at $800 \mathrm{MeV}$. As is the case for the 15.11 MeV state, this transition is mediated by the isovector tensor component of the effective interaction. While it is clear Erom the Elts to the cross section data that the real tensor component dominates, it is interesting to note the relative strength of these components vis-a-vis that observed for the 15.11 MeV state. Whereas the central term dominated almost completely except for large momentum transfer in the case of the $15.11 \mathrm{MeV}$ state, we find here 


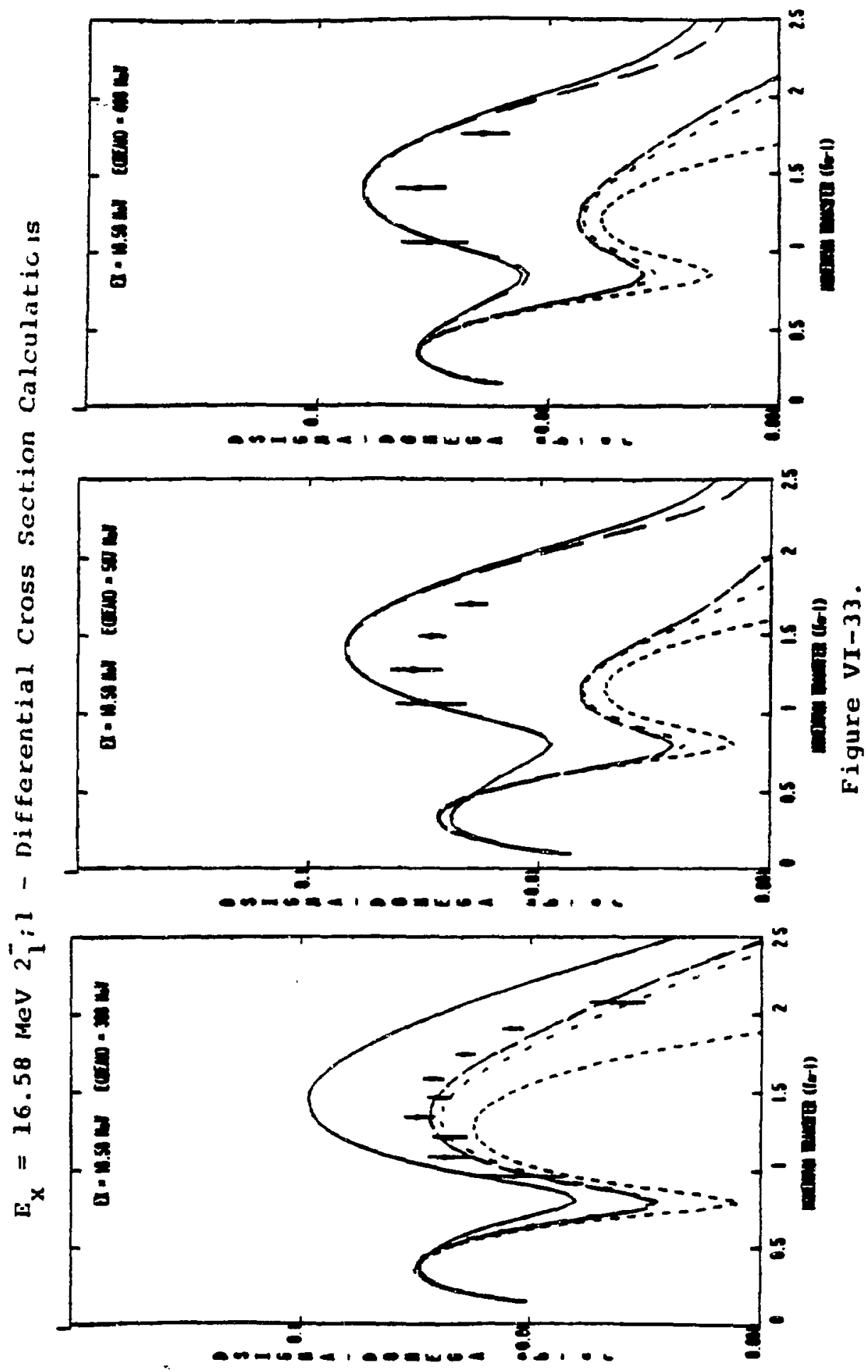




$$
E_{X}=16.58 \mathrm{MeV} 2-; 1 \text { - Analyzing power Calculations }
$$
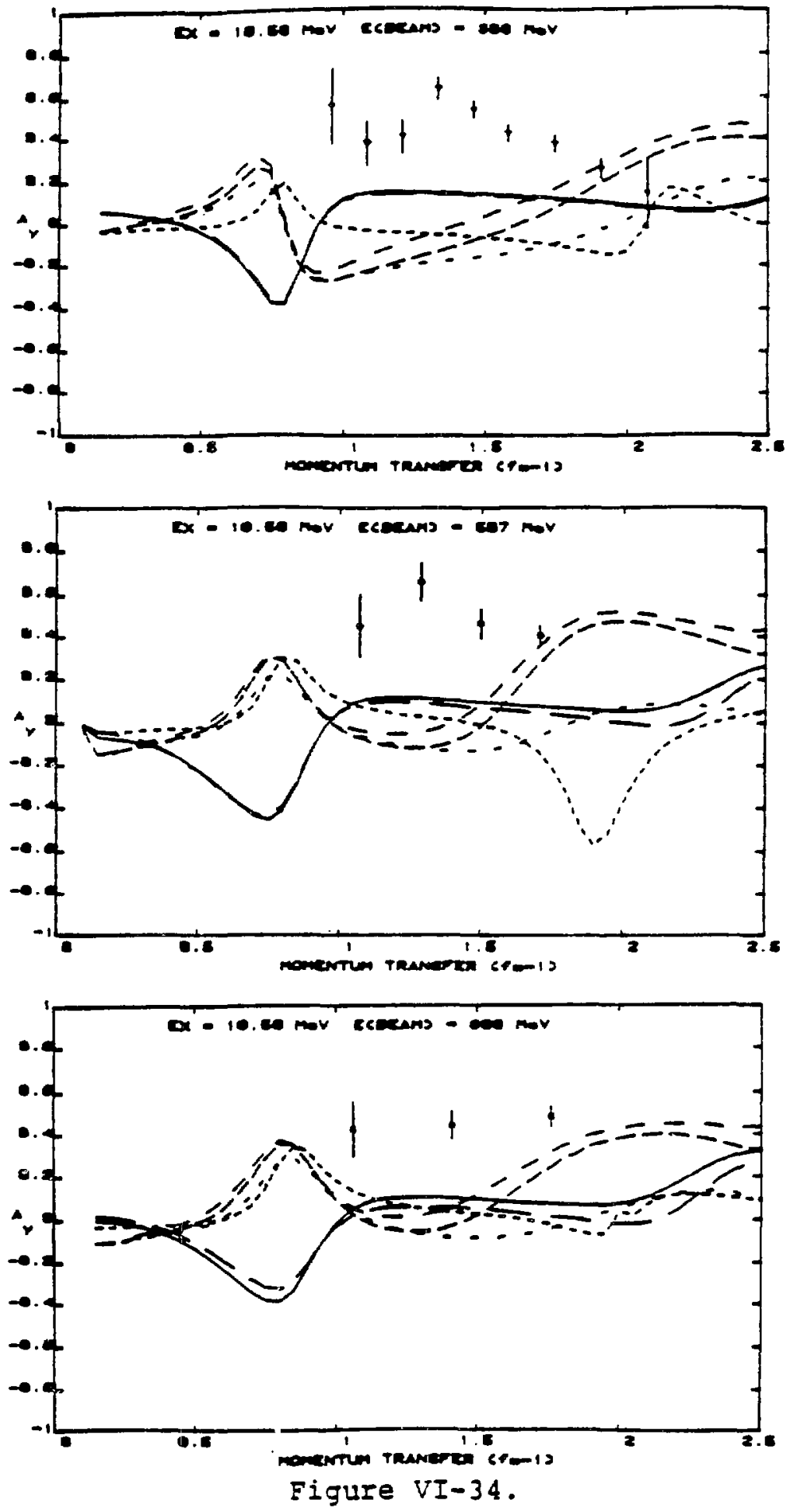

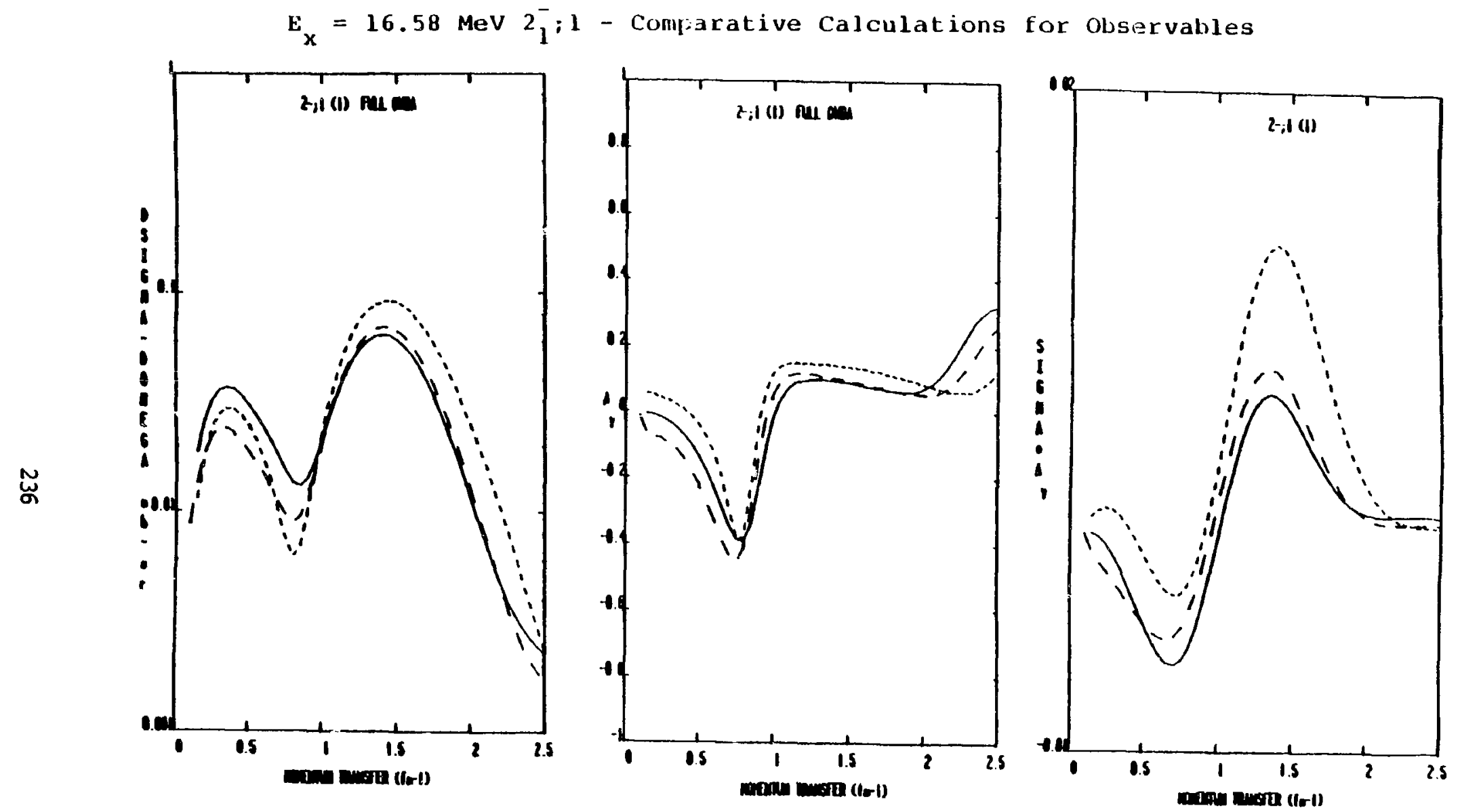

Figure VI-35. 
$E_{x}=16.58 \mathrm{MeV} 2_{1}^{-} ; 1$ - Renormalized Differential Cross Sections
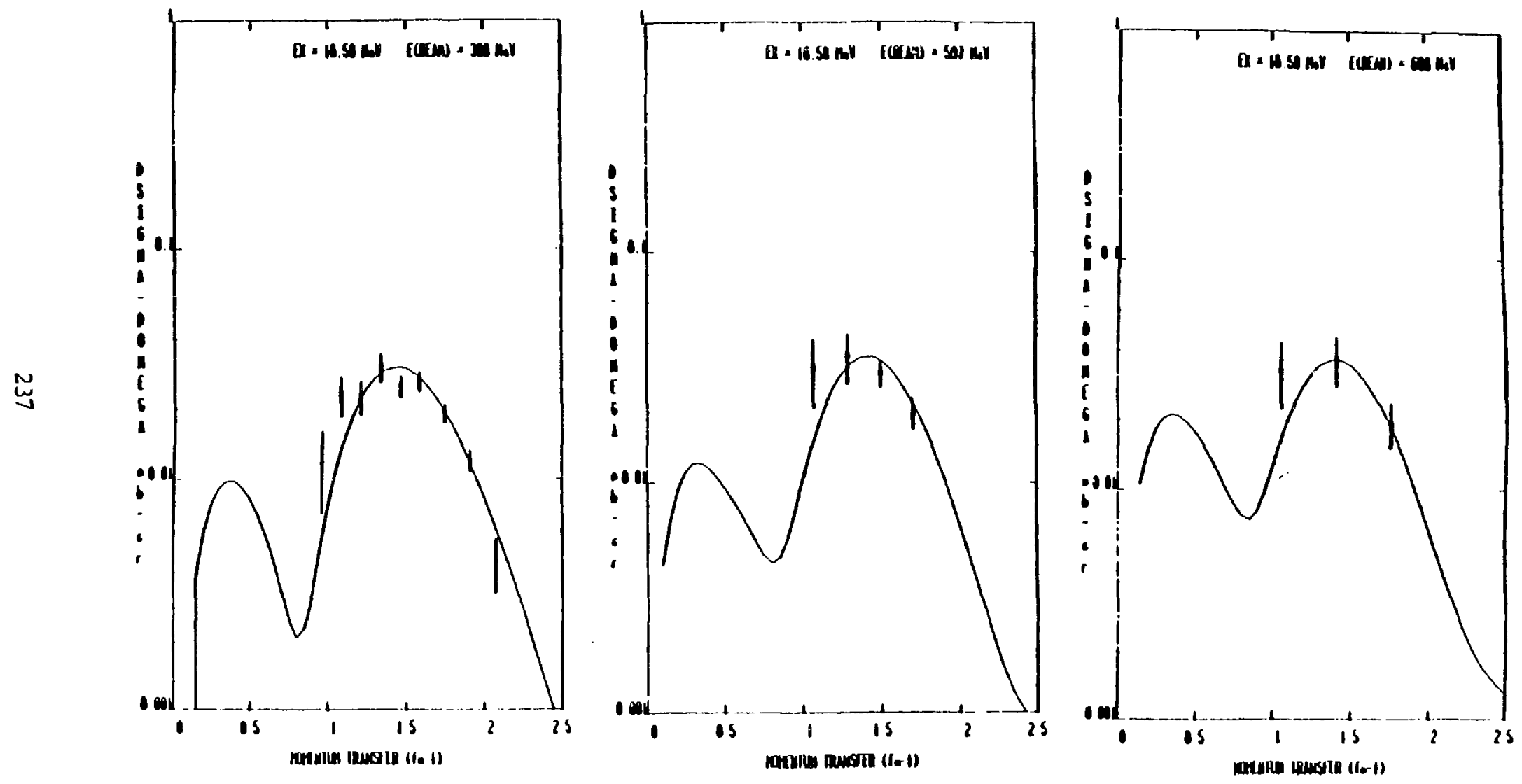

Figure VI-36. 
that the contributions from the tensor term, especlaily the real direct tensor component, are much more significant. Furthermore, while the first maximum is always dominated by the real central term, the second maximum is dominated by the tensor term as was observed for the $15.11 \mathrm{MeV}$ state. Aiso, the 650 MeV force gives a much reduced contribution from the reai central term to the second maximum, and requires iess renormalization to reproduce the data. We note that the $800 \mathrm{MeV}$ data, while requiring no renormalization, show an offset in the maximum which is attributable to the different oscillator parameter used at that energy. Our osclilator parameter would glve a much improved flt to the data.

The structure of the cross section is the result of a sensitive canceliation of the large p-s ampitude with the $p-d$ amplitude at smali momentum transfers, and $L=1$ transfer to the nucleus dominates. From the jocation of the principai maximum, it might be expected that $L=3$ transfer wouid be important, but comfort ${ }^{9}$ ) has shown that this is not the case. In comparing renormaiization factors dertved for $\left(D, p^{\prime}\right)$ scattering with shose for (e,e') scattering53,54) we find that electron scattering requices a much smali renormaitization factor of 0.88 , much more consistent with that for $800 \mathrm{MeV}$ rather than those obtained in this work.

The shape of the calculated analyzing powers are in good agreement with similar cabculations at $122^{7}$ ) and 200 
$\left.M e v^{9}\right)$, and is somewhat reminiscent of that for the $1^{+}$state at $15.11 \mathrm{MeV}$. The data are not fitted at ail, although the shape is consistent. The interference minimum is caused by the direct tensor term, which is generaily responsibie for the shape.

It is diffleult to believe that the deviations from the data are an artifact of the force, since the dominant components in this transition do a reasonabie job os describing the excitation of the 15.11 Mev state. Curiously, the shape at high q here is iell degcribed while that of the 15.11 MeV state is not. Also, the data are overestimated while the 15.11 MeV data are underestimated. Rather, the difficulty may lie with the wave function and the need for a very delicate balance between the two primary configurations contributing to the transition density.

The effects of distortions were not investigated for this transition, and the contributions from knock-on exchange were found to be smali.

5) States for $E_{x}>18.0 \mathrm{MeV}$

(a) The state at $\Sigma_{x}=18.30 \mathrm{MeV}$

In Chapter $v$, it was postuiated that the excitation of this state was isoscalar. Calcuiations were performed for the first three $2^{-} ; 0$ states dertved by Millener, and also for the first and second $1^{-} ; 0$ state. The $1_{1}^{-} ; 0,2 ; 0$ 
and $2 ; 0$ states did not reproduce the shape of the differential cross section at all, showing peaking at larger momentum transfers reminiscent of the structure of the $2^{-} ; 1$ ralcuiations for the $16.58 \mathrm{MeV}$ state. A good flt to the data was found for the $2_{2}^{-} ; 0$ siate, which is dominated by the $\left(d_{5 / 2}, p_{3 / 2}^{-1}\right)$ configuration with non-negilgible admixtures of the $\left(2 s_{1 / 2}, 1 p_{3 / 2}^{-1}\right)$ and $\left(d_{3 / 2} p_{3 / 2}^{-1}\right)$ conflgurations. Th1s is in contrast to the other $2^{-} ; 0$ states and the $2_{1}^{-} ; 1$ state which are primarily dominated by the $\left(2 s_{1 / 2}-p_{3 / 2}^{-1}\right)$ configuration.

The fits to the data are presented in Figures VI-37 and VI-38, while the comparative plots of the caicuiations are shown in Figure VI-39.

We note that the low $q$ dependence, including the $\max : \pi \mathrm{um}$ at $q=0.6 \mathrm{fm}^{-1}$, is well reproduced, but overestimated by a factor of about 1.7 to 2 for all three lncldent particle energies. The principal component of the effective interaction responsible for the excitation of this state is seen to be the real spin-orbit term, primarily through the direct Interaction. Enhancements due to the tensor terms, especialiy at 597 and $698 \mathrm{MeV}$, are signifleant. Knock-on exchange processes provide some small canceliations resulting in an overall geduction of the cross section from the dicet calcuiations.

We note from Flgure VI-39 that the calcuiated cross sections are reasonabiy invarlant with energy except in the 

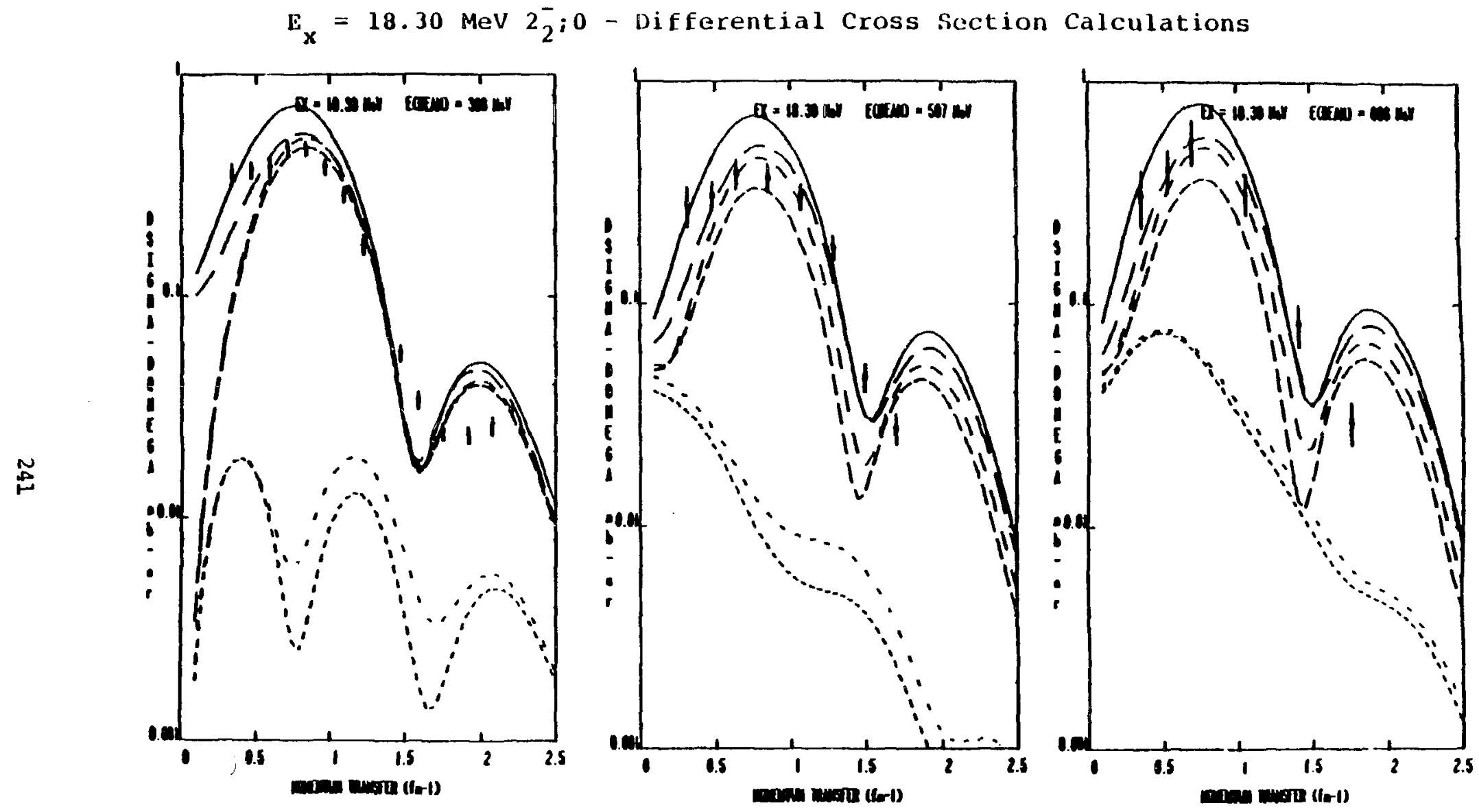

Figure VI-37. 
$E_{X}=18.30 \mathrm{MeV} \mathrm{2;} ; 0$ - Analyzing Power Calculations
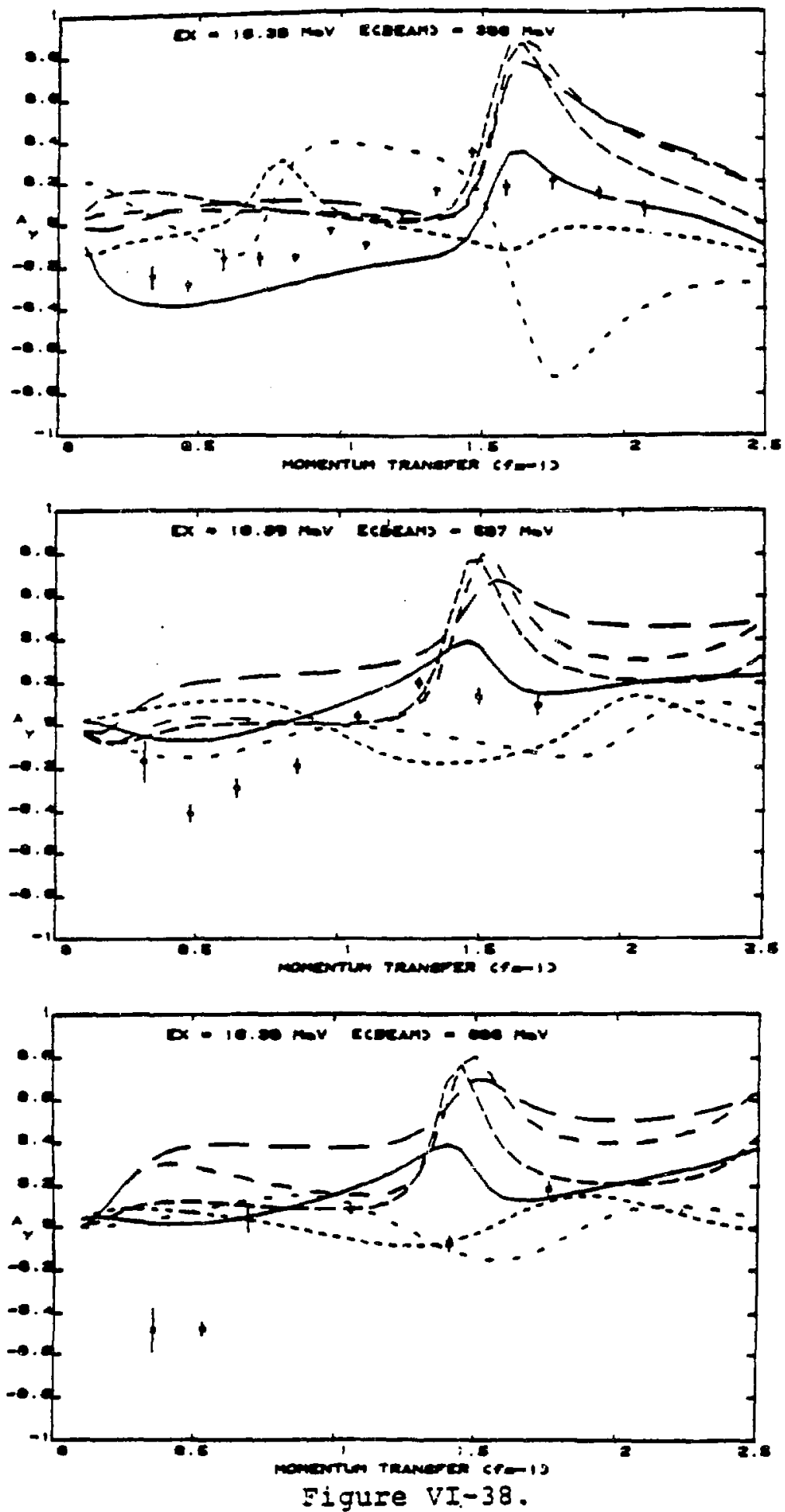

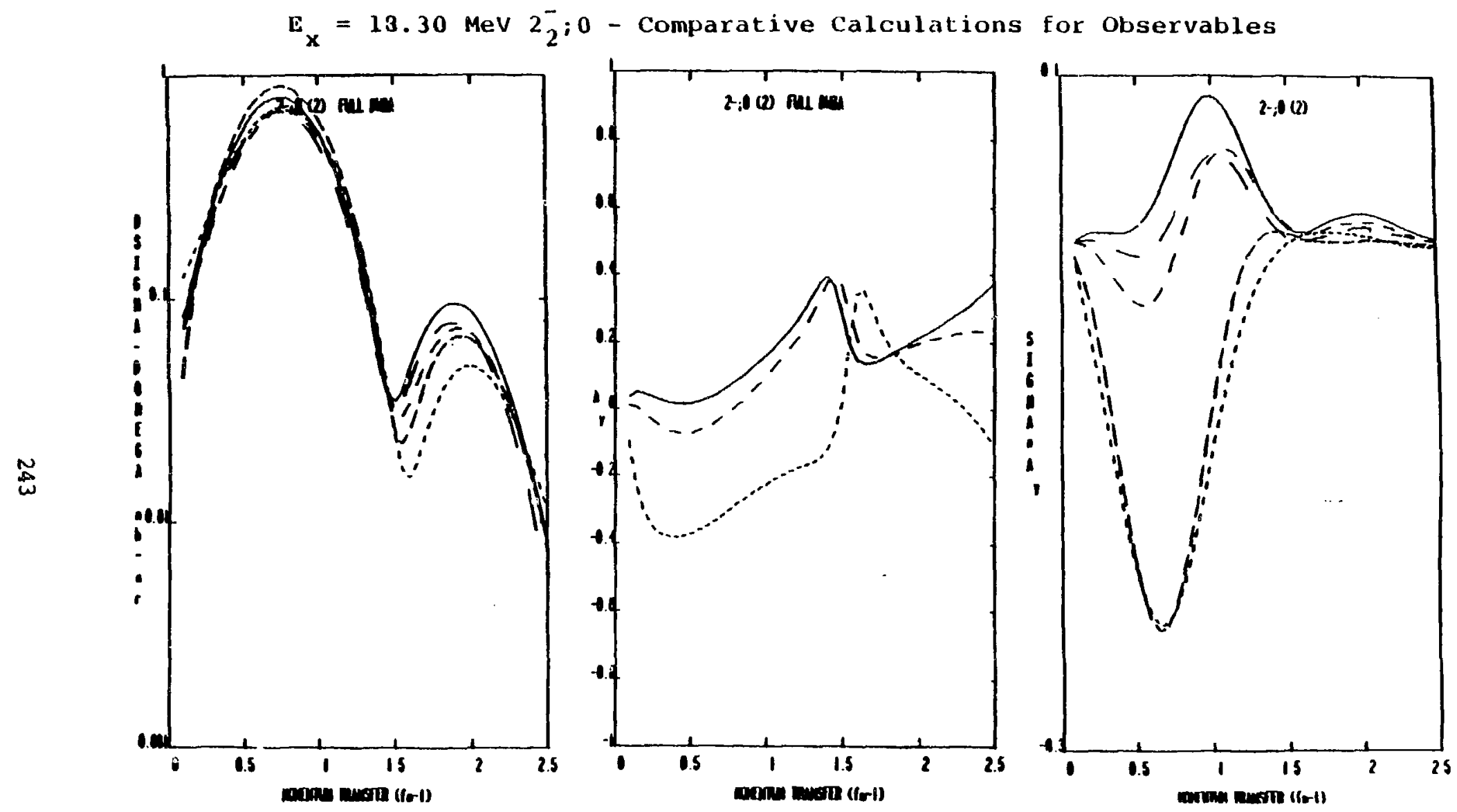

Figure VI-39. 
region of the second maximum. Such behaviour is characteristlc of the unnatural parity nature of the transition, and is also consistent with the energy independence of the data.

The Elts to the analyzing powers show the characteristic negative values for low momentum transfer which we expect from the isoscaiar nature of the excltation. The shape of the data for $E_{p}=398 \mathrm{MeV}$ is very weli reproduced, if somewhat off in magnitude. The fits for the higher beam energies are not as good, showing the same deflciencles as are seen for the $1^{+} ; 0$ state at 12.71 MeV. The tensor term again seems to be important in the generation of the shape of the calculated analyzing power. It should be kept in mind that interference effects make specific ldentification of dominant terms difficult. Signiflcant deviations from beam energy to beam energy are seen for higher momentum transfers. The data and calculations presented here are in excelient agreement with the data obtalned by Comfore et. al. at $200 \mathrm{MeV}$ for a state at $E_{X}=18.40 \mathrm{MeV}^{9}$ ).

Several states are thought to exist near this excltation energy. Karp ${ }^{27}$ ) has shown that a $3^{-}$; 1 state domInates proton-transfer reactions to this excitation region, while Buenerd et. al. 60$)$ found weak evidence for a $2^{+}$; 0 and $3^{-} ; 1$ state $1 n^{\prime}\left(\alpha, \alpha^{\prime}\right)$ and $\left(p, p^{\prime}\right)$ scattering. The sheil-model caicuiations of Cohen and Kurath, Mlilener and Miliener and kurath 61 ) aIso predict many levels near this exeitation. 
Comfort et. al. have performed calculations for the third $2^{+} ; 0$ Cohen-kurath wave Eunction. the lowest $3^{-}$il Milienerkurath wave function and the second $2^{-} ; 1$ Miliener-kurath wave Eunction'). None os these transitions were found to well reproduce elther the differential cross sections or the analyzing powers. The $2^{-} ; 1$ was found to be reasonable in shape but overestimated the cross section by a Eactor of 10. It shouid be noted that at 200 MeV $A_{Y}$ is not as discriminating concerning the isospin character of a transition as it is in this work. Comfort has since affirmed that the 2 ; wave Eunction used in this work yields an improved flt to the data at $200 \mathrm{MeV}^{62}$ ).

As was mentioned in Chapter I, $180^{\circ}$ electron inelastic scattering selects primarily spin- and lsospln-fidp transitions, and can trus be expected to be insensitive to states with $T=0$. Flanz et. al. 24) have studied this regton in such seattering and observe no strength between 18 and $19 \mathrm{MeV}$ of excitation, consistent with the absence of $T=$ 1 states in this region.

In conclusion, on the basis of the systematics of the energy independence of the differential eross section, the shape of the aralyzing power and the quality of the DWIA Eits, together with the evidence Erom electron scattering, we propose that this transition is an unnatural partty isoscalar transition of spin and partey $2^{-}$. We cannot, however, rule out the existence of other weakly exclted states in 
chis same region that are difficult to observe.

(b) The states at $E_{x}=19.28 \mathrm{MeV}$ and $19.65 \mathrm{MeV}$

These states are part of the complex set of overiapping states in the $19 \mathrm{MeV}$ excitation region, and are very cifficult to interpret. Inelastic pion scattering with both $\pi^{+}$and $\pi^{-}$probes provides strong evidence for the existence of a strongiy isospin-mixed pair of $4^{-}$states at excitation energies of 19.25 and $19.65 \mathrm{MeV}^{25,26)}$. The state at 19.25 Mev, which is thought to be dowinantly $T=0$ is strongiy excited in ineiastic $\pi^{+}$scattering, while that at $19.65 \mathrm{MeV}$ which is thought to be dominantiy $T=1$ is strongly excited in ineiastic $\pi^{-}$scattering,

Inelastic election scattering at $180^{\circ}$ provides Euther infornation which seems oniy to cloud the issue. Fianz et. ai. 24) ciaim to see three states in this region, at $E_{X}=19.25,19.50$ and 19.65 Mev. The interpretation of these states is as follows. The $19.65 \mathrm{MeV}$ state form- factor is well-reproduced by a 4 il $l$ transition density, while the weak 19.5 MeV state is consistent with a $4_{1}^{-} ; 0$ wave function. The form factor for the 19.28 MeV state is best described by a combination of $2_{2}^{-} ; 1$ and $1_{2}^{-} ; 1$ strength at low and high momentum transfer respectiveiy. It is possibie to observe $T=0$ strength in electron backscattering if appreciabie isospin mixing is present. It is clear that the ineiastic pion and ejectron scattering resuits are not entirely 
compatible.

How then might the ineiastic proton seattering data presented here be interpreted? We have used the spin and isospin assignments of both $\left(\pi, \pi^{\prime}\right)$ and $\left(e, e^{\prime}\right)$ scattering as a guide, and computed the expected differential cross sections and anaiyzing powers for $4_{1}^{-} ; \theta, 4-i, 2_{2}^{-} ; 1, I_{1}^{-} ; 1$ anci $1_{2}^{-} ; 1$ shell-model states derived by Miliener. Several conciustons may be drawn. The anguiar aistribution of the $2^{-} ; 1$ state is quite incompatible with the data, and is consequentiy discarded. The $4_{1}^{-} ; 0$ state is found to reasonabiy reproduce the magnitude of the differential cross section for the $19.28 \mathrm{MeV}$ state, requiring a untform downward renormaitization of about 1.7, while the shape of the $19.65 \mathrm{MeV}$ state was reasonably reproduced by the $4_{1}^{-} ; 1$ caicuiation. The transition densities for these two states are pure $\left(d_{5 / 2} \mathrm{p}_{3 / 2}^{-1}\right)$ configurations. On the other hand, both the $1_{j}^{-} ; 1$ and $1-i j$ states have a second maximum at $q=1.5 \mathrm{fm}^{-1}$ which only underestimate the data for the 19.28 MeV state by factors of two to three. The strength for both these states that is predicted at $q=0.6 \mathrm{fm}^{-1}$ is not seen in the data; 1f it is there, it is coneivabie that it is masked by the strong excitation of the $19.49 \mathrm{MeV}$ state, especialiy constdering the width of the $19.28 \mathrm{MeV}$ state. The $1_{2}^{-}$il state is possibly more likely, based on It's much reduced forward angie strength with respect to the flist $1^{-} ; 1$ state.

The calculations for the $4^{-}$states are presented in 
Figures VI-40 to VI-45, while those for the seconi 1 ; 1 state are shown in Figures VI-46,47. The caicuiated $4^{-} ; 1$ cross sections for the $19.65 \mathrm{MeV}$ state require renormajization by factors of $3.33,0.6$ and 0.4 to reproduce the magnitudes of the data for incident energles of 398,597 and 698 Mev respectiveiy.

We note that the excttation of the $4_{1}^{-} ; 0$ stato is dominated by the reai direct part of the spin-orbit interaction, with some small enhancement due to the tensor exchange terms. This is in keeping with the effects observed for the $2^{-} ; 0$ state at $18.30 \mathrm{MeV}$ discussed previousiy. The excitation of the 19.65 Mev state is dominated by the reel direct tensor term with some "gh momentum transfer enhancements due to the spin-oroit and central terms. Rnock-on exchange effects are lound to be smail. The large contributions from the tensor term is in keeping with the effects seen for the $2^{-}$il stace at $16.58 \mathrm{Mev}$.

The fits to the anaiyzing power are very reasonabie for the $19.65 \mathrm{MeV}$ state. The snape is weil-reproduced but the magnitude is generally underestimated. The structure of this observabie is influenced by the tensor component of the force. The $4_{1}^{-}$; fits to the analyzing power data for the 19.28 MeV state are legs successful, but the relative scarclty of data points makes Interpretation diffeult. The negative character is reproduced for the $398 \mathrm{MeV}$ and $597 \mathrm{MeV}$ data, but not for the $698 \mathrm{MeV}$ data. The calculations show 
$E_{x}=19.28 \mathrm{MeV} \overline{4}_{1} ; 0-D i f f \in r e n t i a l$ Cross Section Calculations
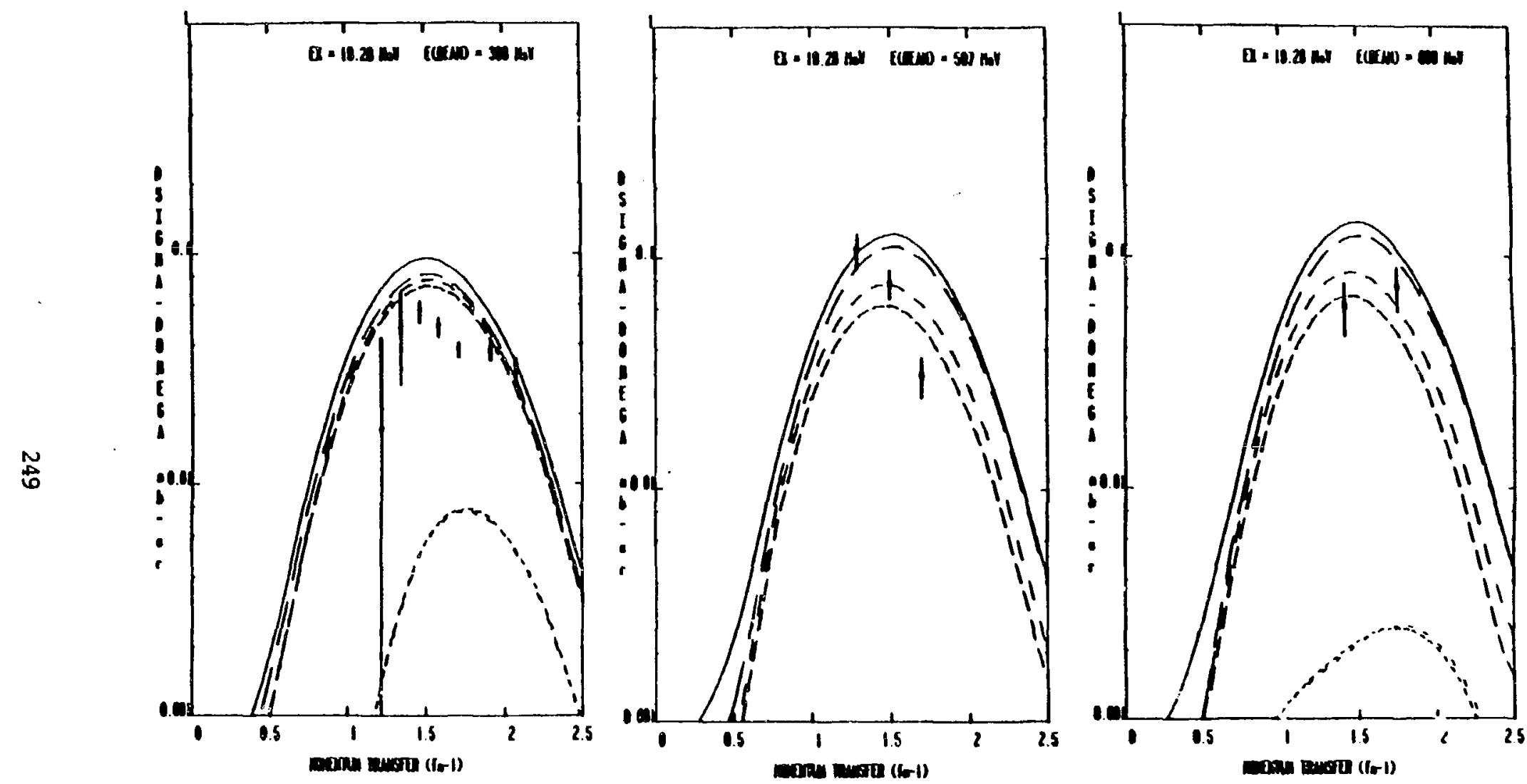

Figure VI-40. 
$E_{x}=19.28 \mathrm{MeV} 4_{1}^{-} ; 0$ - Analyzing Power Calculations
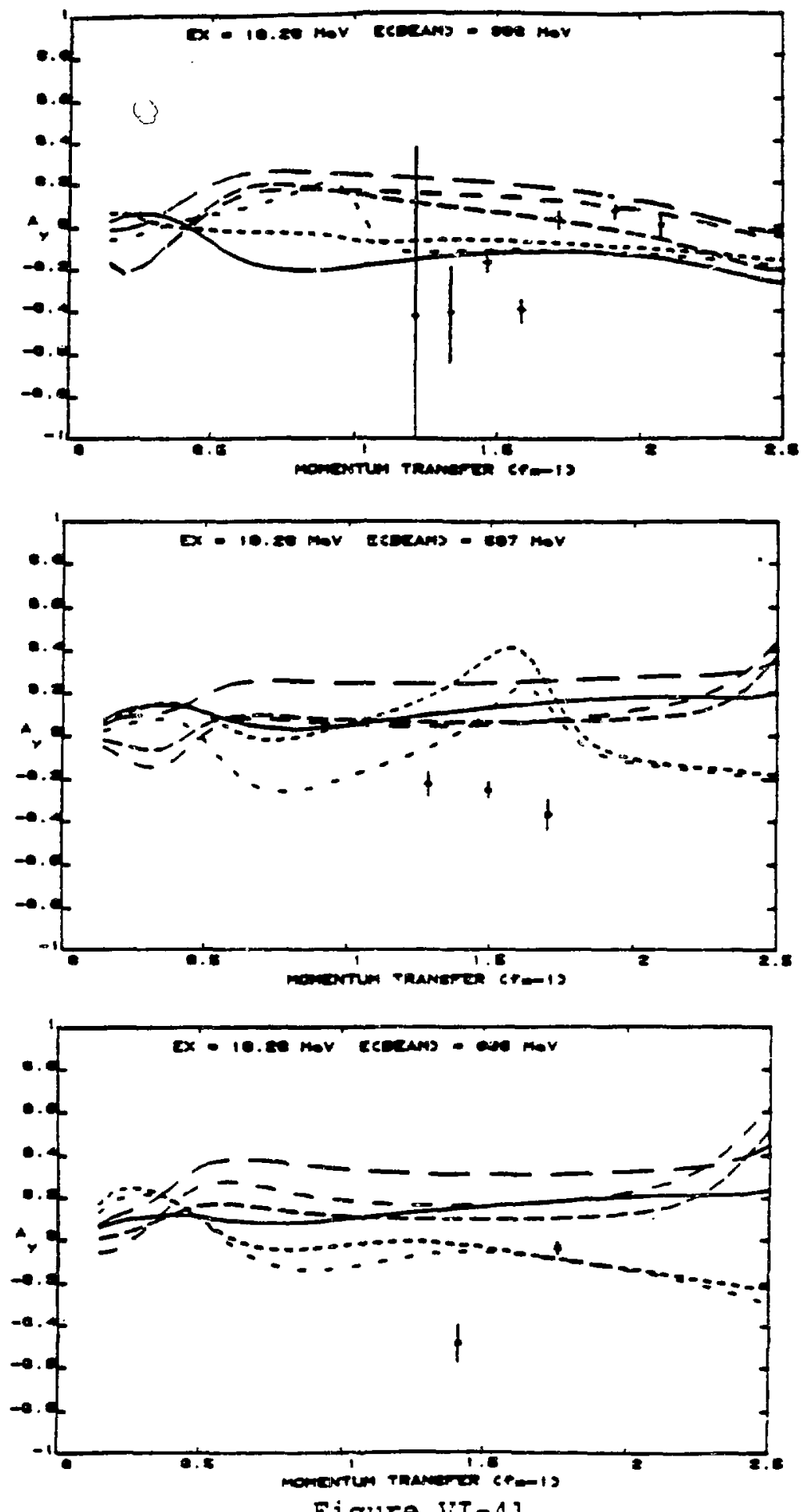

Figure VI-4l. 

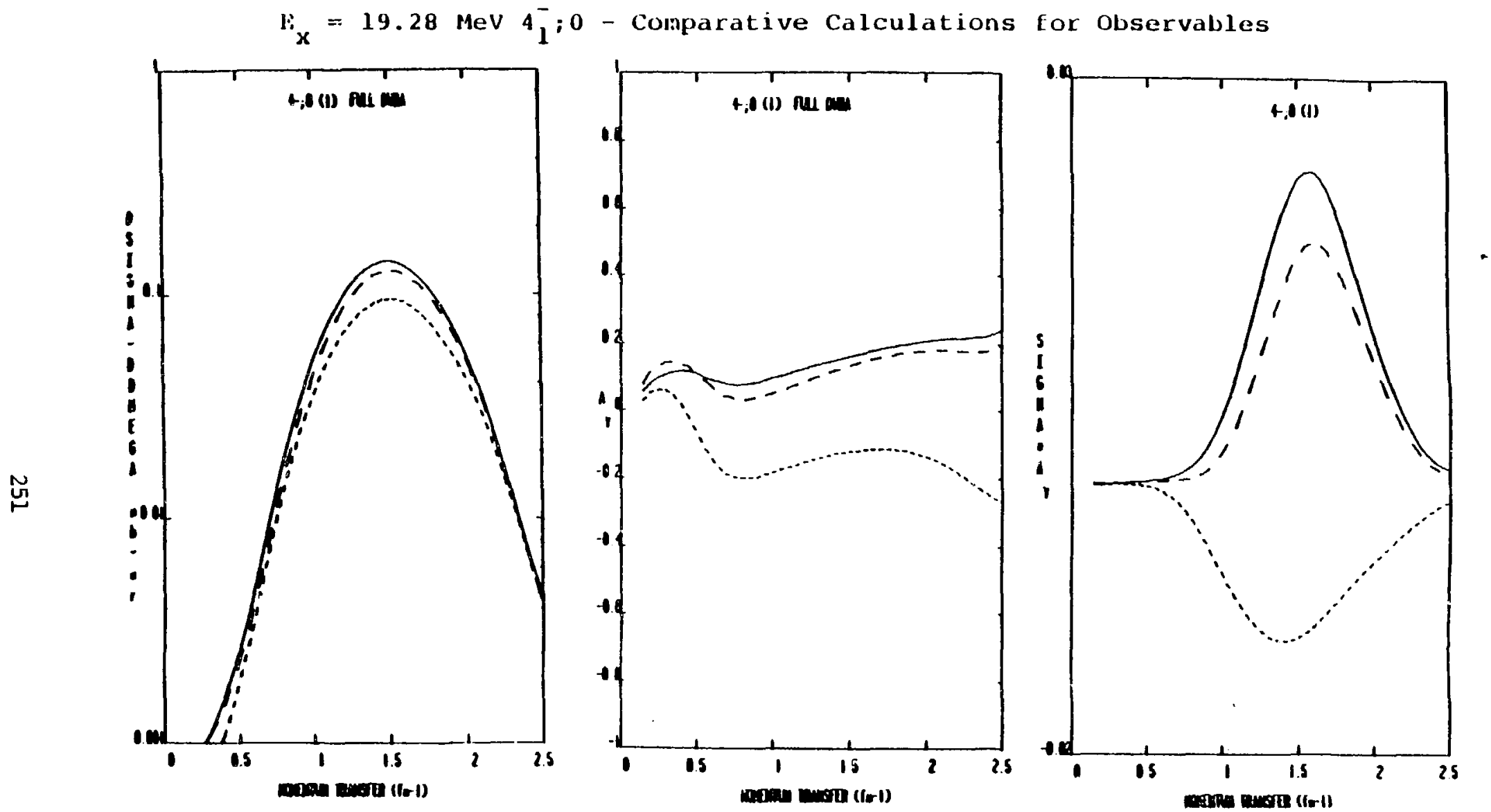

Figure VI-42. 
$E_{x}=19.65 \mathrm{MeV} 4_{1}{ }^{i}$ - Differential Cross Section Calculations
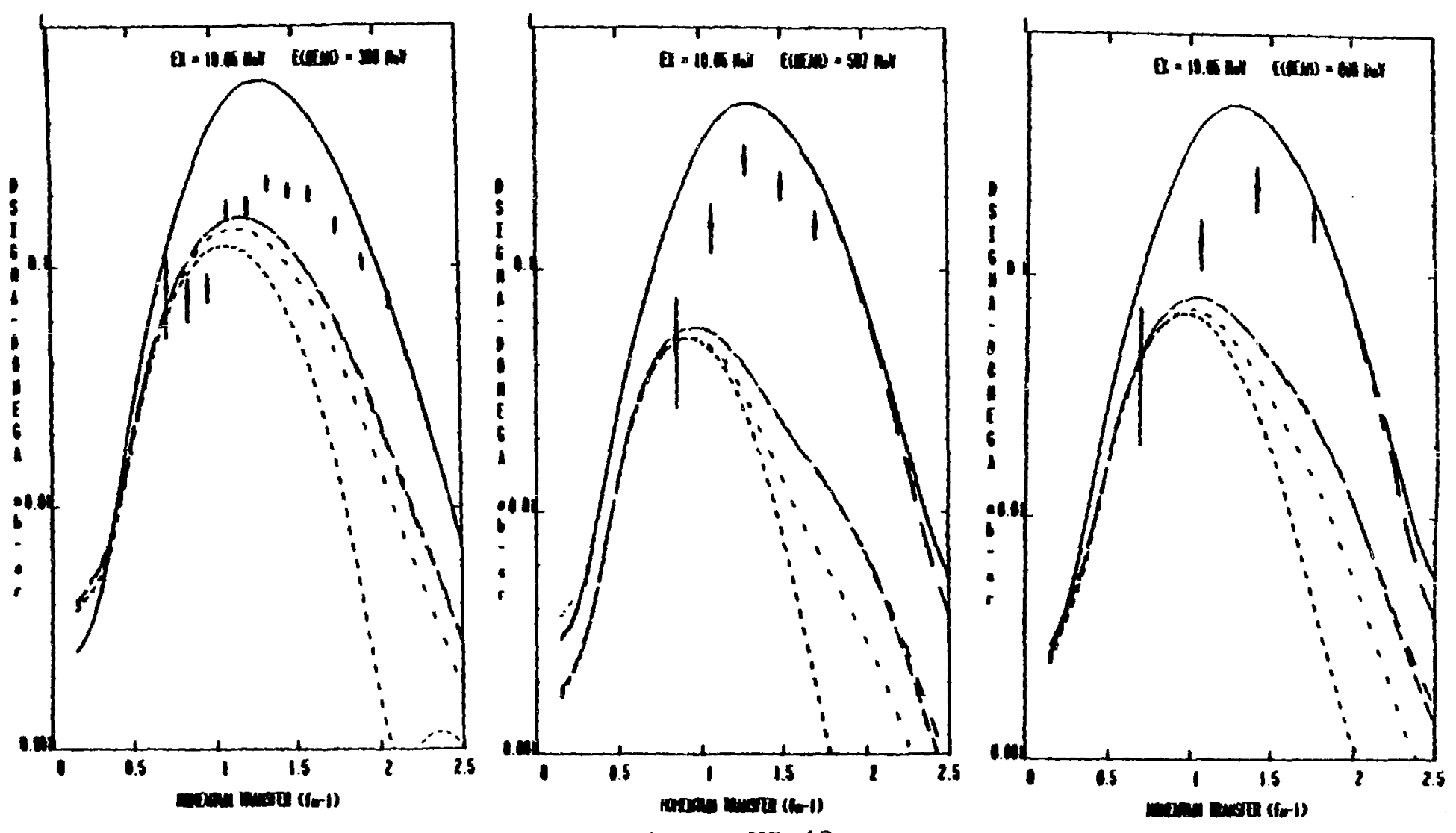

Fi qure VI-43. 
$E_{x}=19.65$ MeV 4j:1 - Analyzing Power Calculations
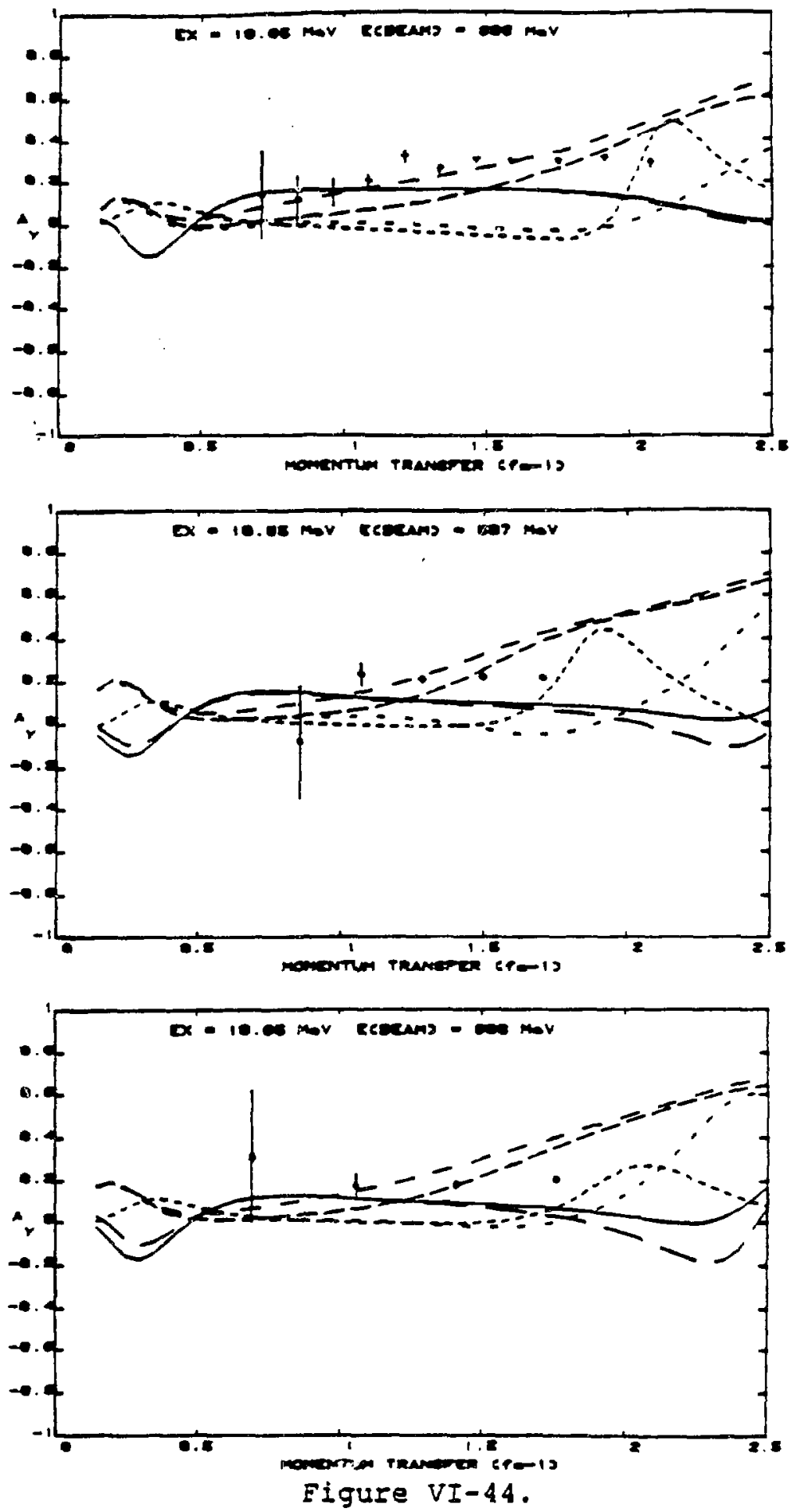
$\mathrm{E}_{\mathrm{x}}=19.65 \mathrm{MeV} 4_{1}^{-} ; 1$ - Comparative Calculations for observables
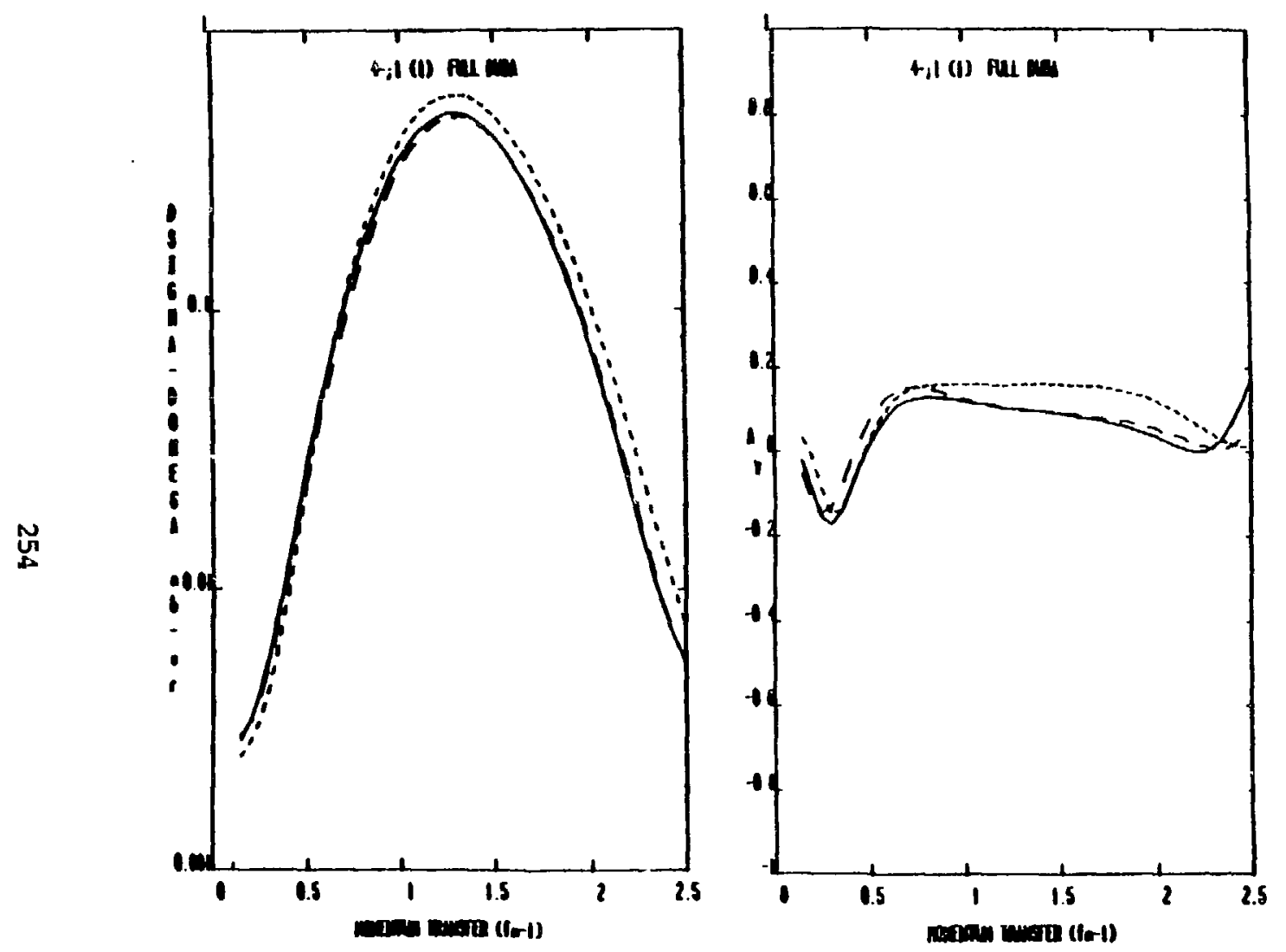

Figure VI-45.

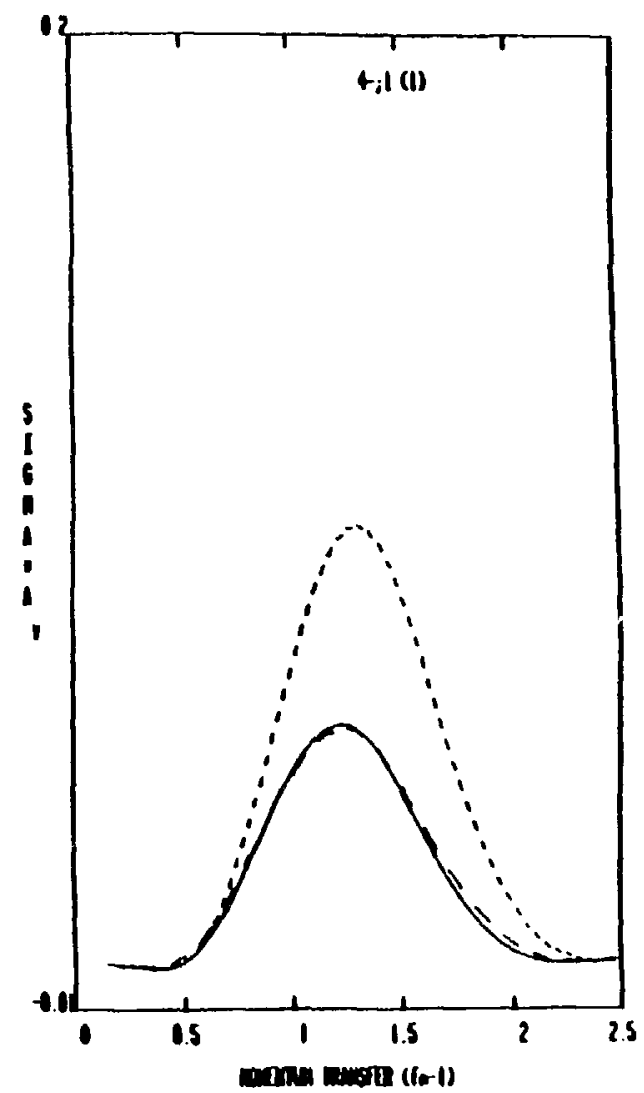


$E_{x}=19.28 \mathrm{MeV} 1_{2}^{-} ; 1$ - Differential Cross Section Calculations
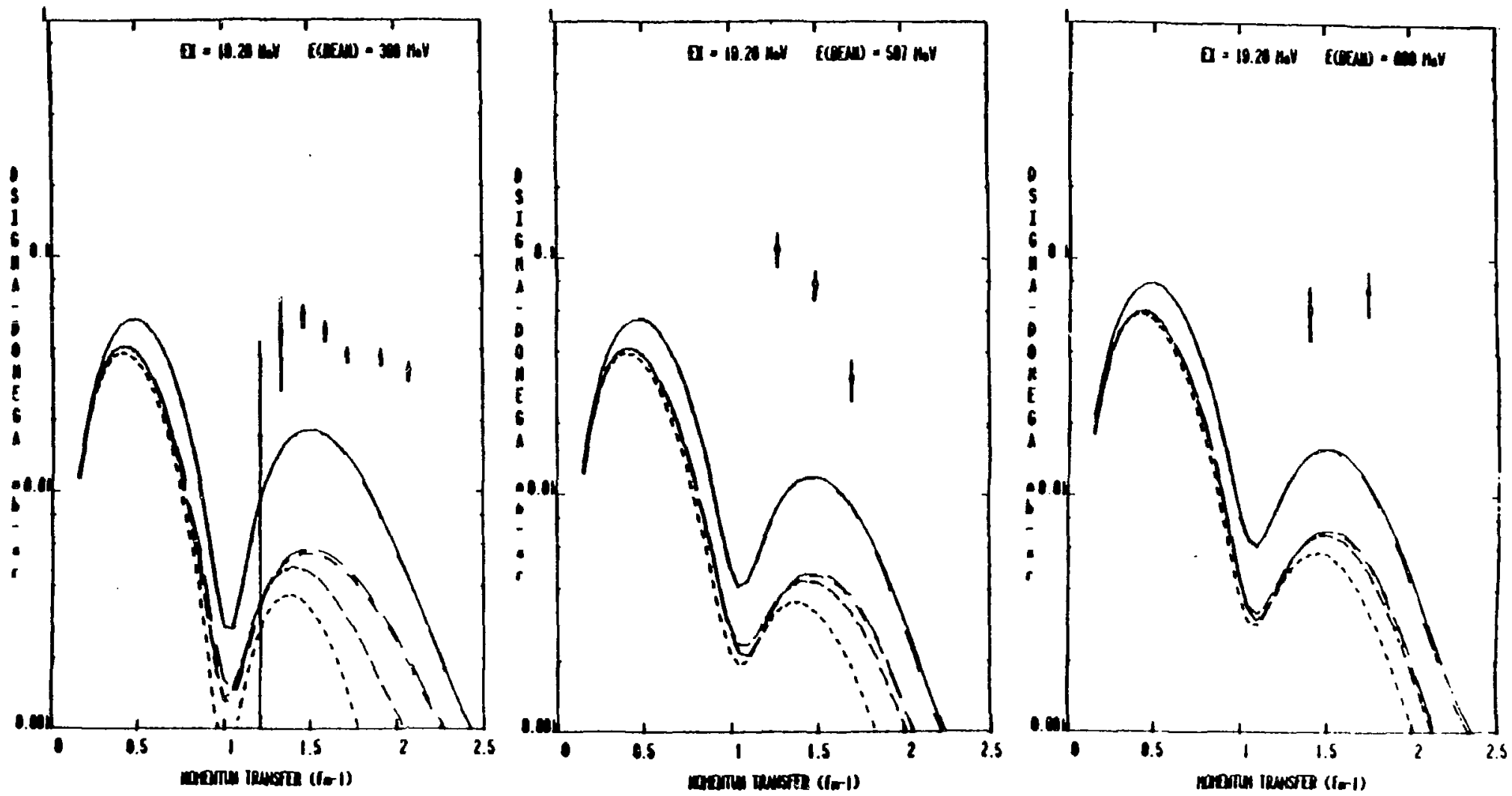

Fiqure VI-4仑. 

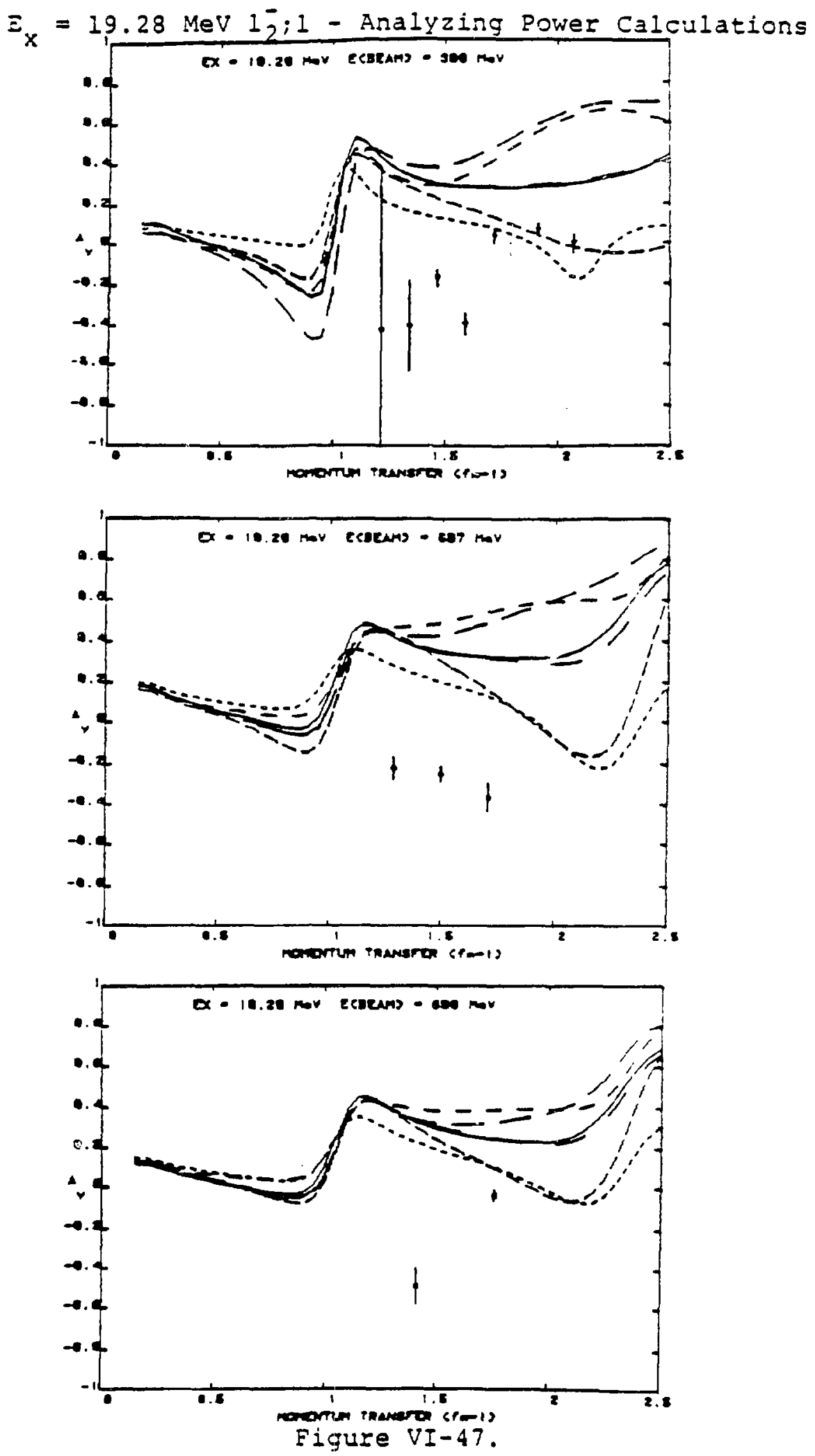
iess structure than other isoscalar unnatural parity transitions, especially at low $q$. In contrast, the $I^{-}$il states predict strong positive analyzing powers in the region where the daca exist, so the $4^{-} ; 0$ computations are in somewhat better agreement with the data.

In summary, we find the 19.28 MeV state to be best represented by a $4^{-} ; 0$ eransition and the $19.65 \mathrm{MeV}$ stare to be best represented by a $4^{-}$; l transition, more in keeping with the results of inelastic pior: scattering than ineiastic electron scattering. It must be stressed, though, that those identiflcations should not be interpreted as absolute. The possibility of the existence of isovector $I^{-}$strength cannot be discounted. More data, especially Ay for the two higher beam energies for $E_{x}=19.28 \mathrm{MeV}$, are required for better comparisons and understanding.

(c) The state at $E_{x}=19.40 \mathrm{MeV}$

This state, which is strongly excited at smaij momentum transfers has been observed in inelastic pion scattering. Although no state of this excitation energy has been seen in electron scattering, we will show that it appears consistent with the low g strength of the $19.28 \mathrm{MeV}$ state observed by Flanz et. al. 24). The inelastic plon scattering data aro strongiy suggestive of lsospin mixing between states observed at 18.4 and $19.4 \mathrm{MeV}$. An assignnent of $2^{-}$for spin and parity was made, and this then suggested 
that the $19.4 \mathrm{MeV}$ state observed in this work might be a $2^{-}$ ; 1 state.

Consequentiy, the calculation for such a transition using Miliener's second $2^{-}$; 1 sheli-model state was made, and the results are presented in Figures VI-48 to VI-50. The dominant configurations for this transition are $\left(d_{5 / 2} p_{3 / 2}^{-1}\right)$ and $\left(d_{5 / 2} p_{1 / 2}^{-1}\right)$ of opposite phasing, together with a contributions from the (2s $1 / 2 p_{3 / 2}^{-1}$ ) configuration. The $1_{1}^{-} ;$calcuiations are shown in Elgures VI-5I to VI-53.

The differential cross section is weij-reproduced in shape, but is overestimated by a factor of 3 for the $2^{-} ; 1$ state. This is reasonable, however, because MiIIener ${ }^{63}$ has shown. that in order to reproduce the transverse electron scattering form-factor for this excitatior to the 19.28 MeV state observed in eiectron scattering, a reduction by a factor of 3 must be applied to the theoretical form-factor. such renormaijzation yieids aimost perfect fits to the data reported here. On the other hand, if the transition is possibiy a $1^{-}$il state, then a minimum renormaiization by a factor of three upwards is required, while no low g strength is seen in electron scattering.

We note that, in keeping with the lower $2^{-}$il state and other isovector unnatural partiy states, the transition is dominated by the real direct tensor term. The lijil state also shows this dependence. Exchange effects result 
$E_{x}=19.40 \mathrm{HeV} 2_{2}^{-} i l-D i f f e r e n t i a l$ Cross Section Calculations
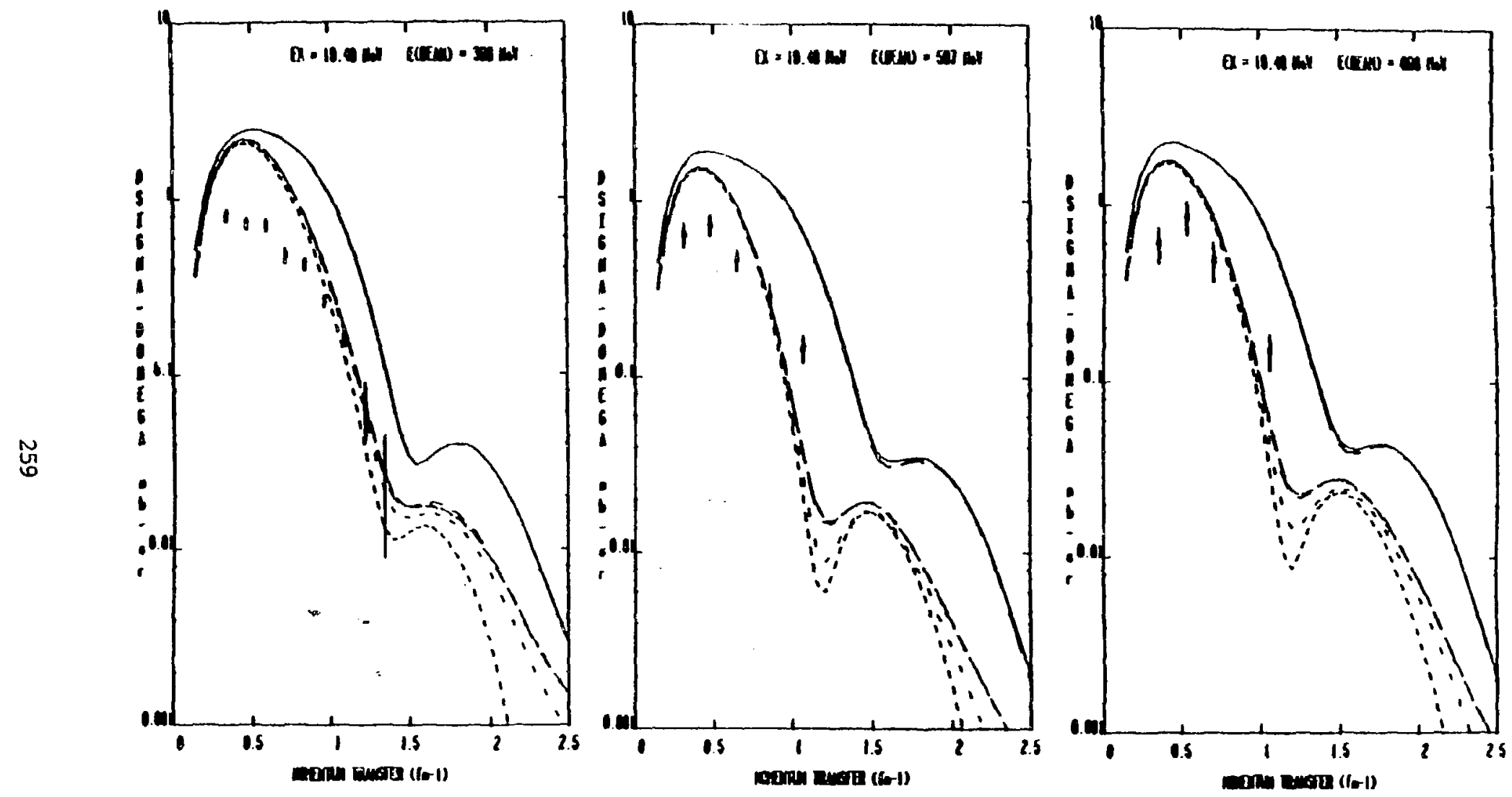

Figure VI-48. 
$\vec{z}_{\mathrm{x}}=19.40 \mathrm{MeV} 22^{-} ; 1$ - Analyzing Dower Calculations
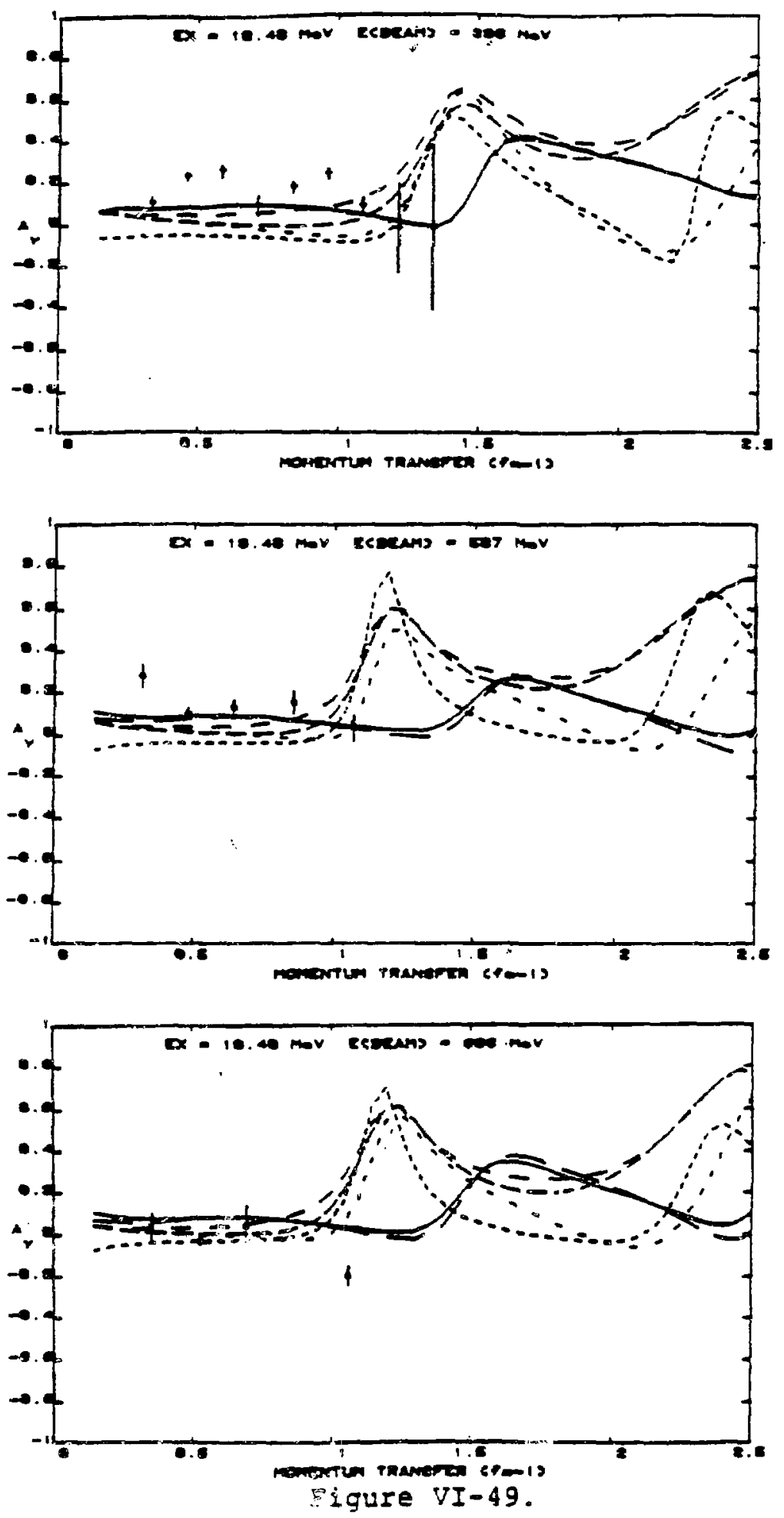


$$
\mathrm{E}_{\mathrm{x}}=19.40 \mathrm{MeV} \mathrm{l}_{1}^{-} ; 1 \text { - Differential cross Section Calculations }
$$
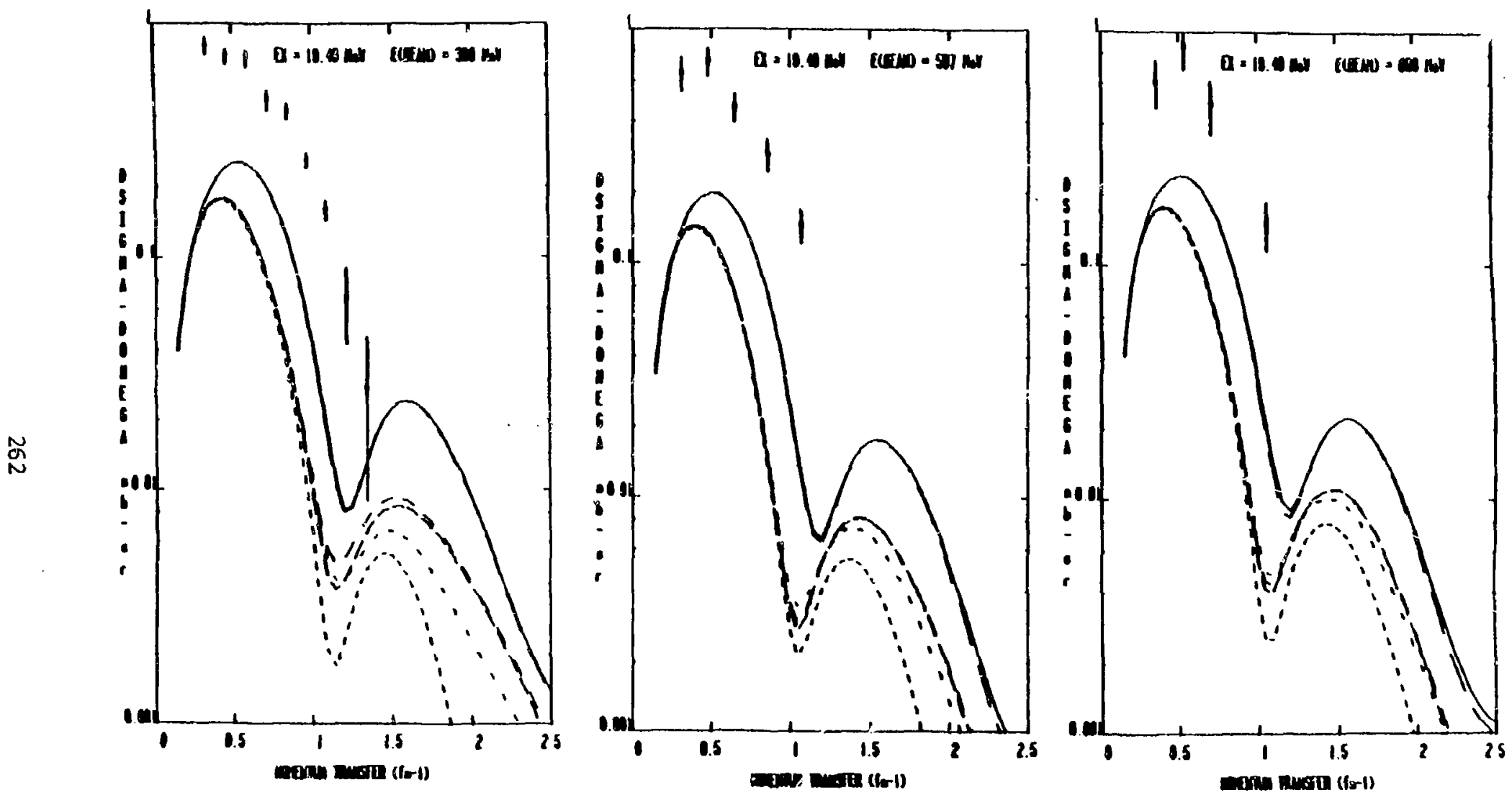

Figure VI-51. 

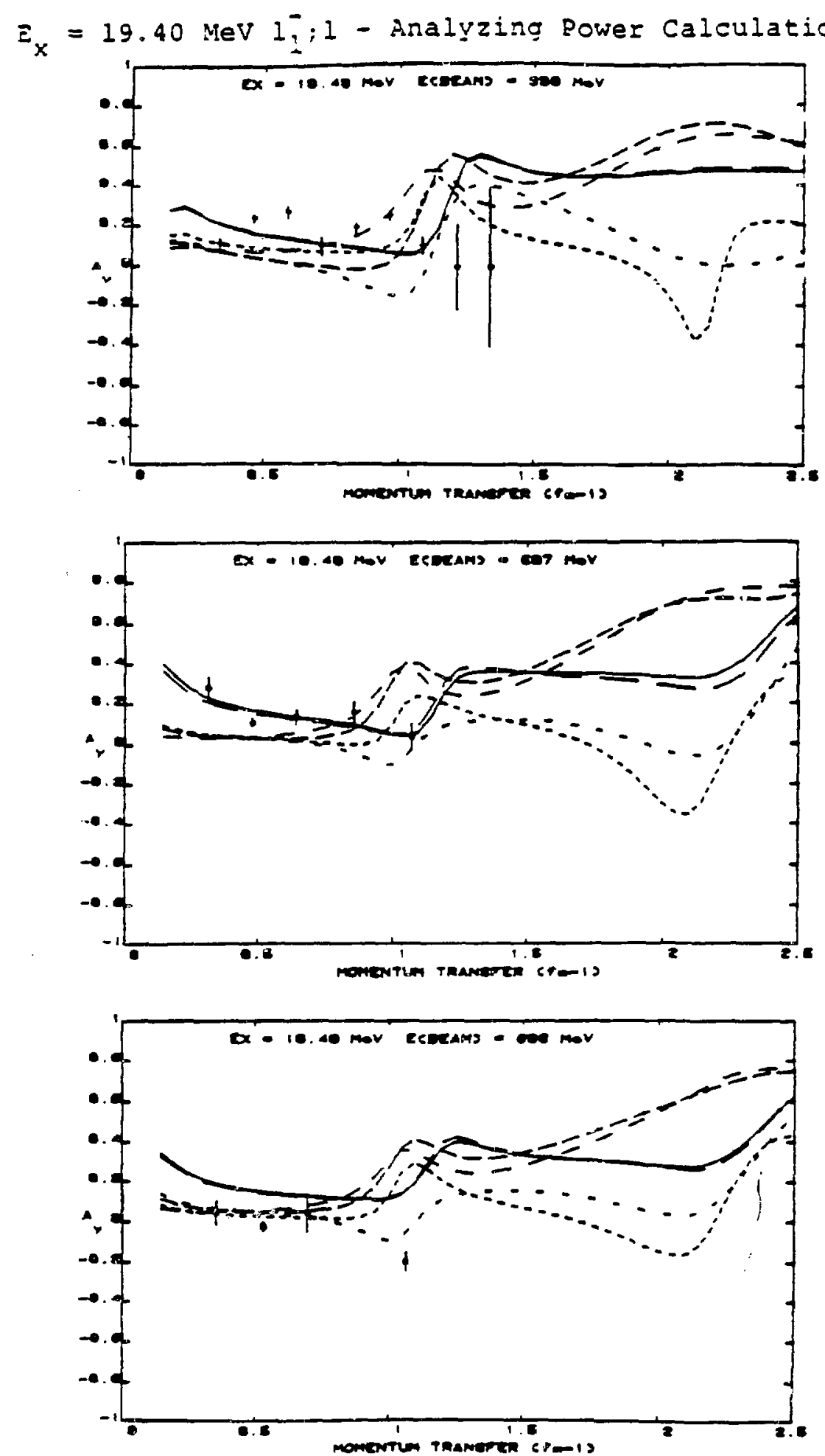

Figuze VI-52. 

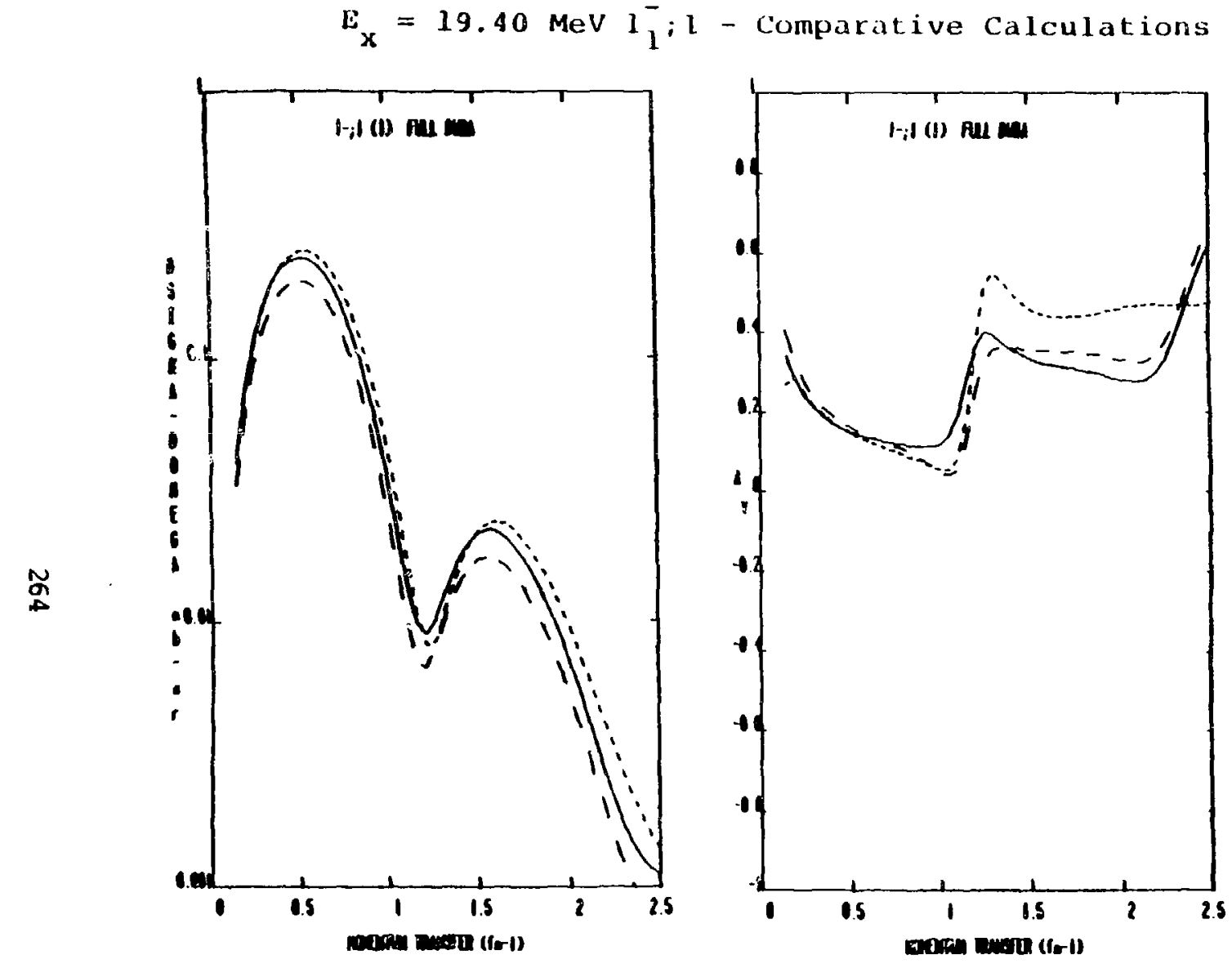

for Observables

Figure VI-53.

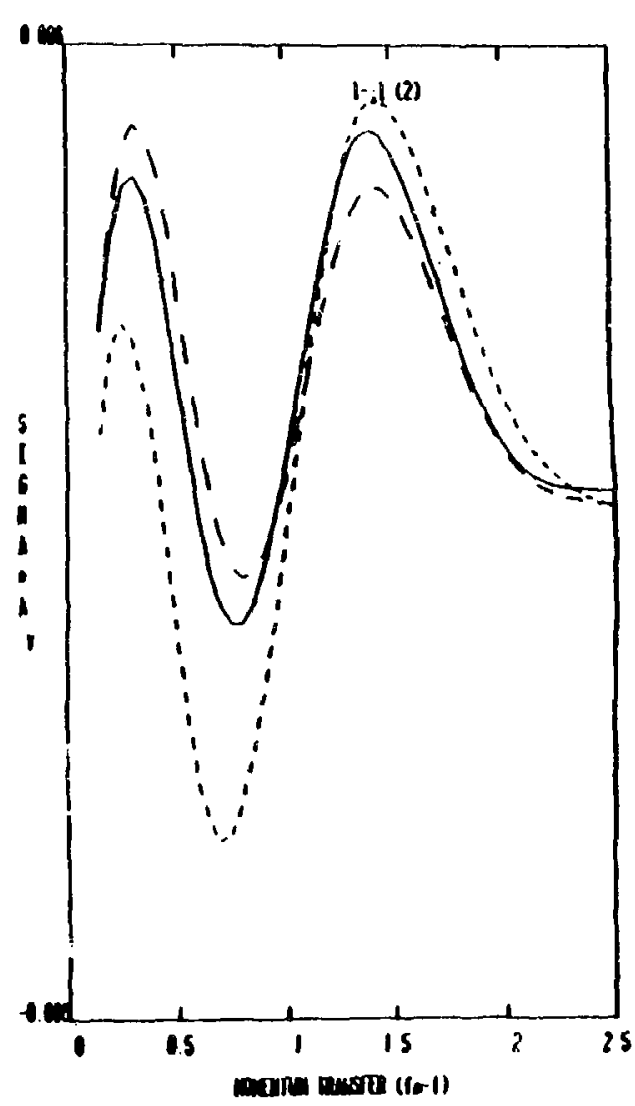


in some destructive interference and consequently a smali reduction in the cross section as caicuiated by pureiy direct processes. Since the data do not extend beyond about $1 \mathrm{fm}^{-1}$ it was not possible to investigate the effects of the contribution of the $I^{-}$; I state at large momentum transfer. It would be interesting to do this to verify the electron scattering results, but the rapid decrease of the cross section in this region makes such experiments very difficuit.

The anaiyzing power data are reasonabiy reproduced by the $2^{-} ; 1$ calculations. The structure of the calcuiations is influenced by the tensor component of the interaction, as we have grown to expect. The dependence of the analyzing power on momentum transfer is consistent with other unnarural parity isnvector states, especialiy the $1^{+} ; 1$ itates at $E_{x}$ $=15.11 \mathrm{MeV}$. However, the data are better reproduced by the $I_{I}$ il analyzing power so discrimination on the basis of $A_{y}$ is difficuit. The energy independence of the differential cross section is further evidence for the isovector character of the state.

Returning to the question ot isospin mixing, some questions become immediately obvious. Morris ${ }^{26}$, has shown that an off-diagonal mixing strength of 350 (40) keV is present for the $2^{-}$states at 18.4 and $19.4 \mathrm{MeV}$ in the inelastic pion spectra. This is almost three times the mixing strength of the $1^{+}$states in ${ }^{12} \mathrm{C}$. Such mixing would then seem to indicate that the $T=\theta$ state should be seen in 
eiectron backscattering because of the appreciable isospin mixing, just as the $4^{-} ; 0$ is observed in $\left(e, e^{\prime}\right)$ scattering, aibeit weakly. However, as has been pointed out previousiy, no strength is seen in the inelastle electron scattering spectrum at this excitation energy. Some strength is seen at $18.80 \mathrm{MeV}$, and Buenerd 64 ) hes reported a state at this energy in $45 \mathrm{MeV}\left(p, p^{\prime}\right)$ scattering, which is not saen at an incident proton energy of $155 \mathrm{MeV}$. These data aiso show significant strength at 18.35 , 19.40 and $19.65 \mathrm{MeV}$, consistent with what is seen in this work. Unfortunateiy, $\left(p, p^{\prime}\right)$ scattering is insensitive to the isospin mixiag, and hence cannot serve as a tool for resolving this apparent discrepancy, which is nevertheless interesting.

In summary, then, a very tentative assignment of spln, parity and isospin of $2^{-}: 1$ can be made for the state at $E_{x}=19.40$ MeV, consistent with plor scattering, but not consistent with the excitation energy.at which $2^{-}$strength is seen in electron scattering. In order to better discriminate between the natural (1-) and unnaturai (2-) partty states in question, spin-Elip probabilities should be measured, but such measurements are especialiy difficuit considering the broad nature and the excitation region of these states.

(d) The state at $E_{X}=20.6 \mathrm{MeV}$

This state has been observed by Comfort et. al. and 
Buenerd et. al. In iower energy ( $\left.p, p^{\prime}\right)$ scattering. There is much conflfcting evidence for spin, parlty and isospin assignments near this excltation energy. Deuteron stripping near this excitation energy indlcates a state of $3^{+} ; 1$, while analogue states in $12_{B}$ indicate the presence of $a 3^{-i}$ states. Buenerd et. al have observed strength in (P, $\left.P^{\prime}\right)$ scattering at 45 and $155 \mathrm{Mev}$, but not in $\left(\alpha, \alpha^{\prime}\right)$ scattering at $60 \mathrm{MeV}$. Reasonable Elts to the $\left(p, p^{\prime}\right)$ data were obtalned under the assumption that the state was $3^{-}$; 1 . Deuteron plek-up reactions indicate the presence of unnatural parity excitations as do the presence of strength for $\left(d, d^{\prime}\right)$ and absence for $\left(\alpha, \alpha^{\circ}\right)$ reactions. The cross section and anaiyzing power data of Comfort et. al. at $200 \mathrm{MeV}$ are in agreement with those reported in this work. The independence of the data with energy are consistent with all the possibilitles presented above.

We have calculated the observables for the iowest $3^{-}$il transition of Miliener, the dominant configuration being $\left(d_{5 / 2} p_{3 / 2}^{-1}\right)$ with a contzibution from the $\left(a_{3 / 2} p_{3 / 2}^{-1}\right)$ conElguration. No calculations were made for a $3^{+}$il state for which we had no wavefunction, nor for any isoscalar states. The resuits of the caiculations are compared with the data in Figures VI-54 to VI-56.

Since this is possibly onjy the second natural parity isovector state studied, it is gratifying to find the o alitative features of the calculated observables to be 


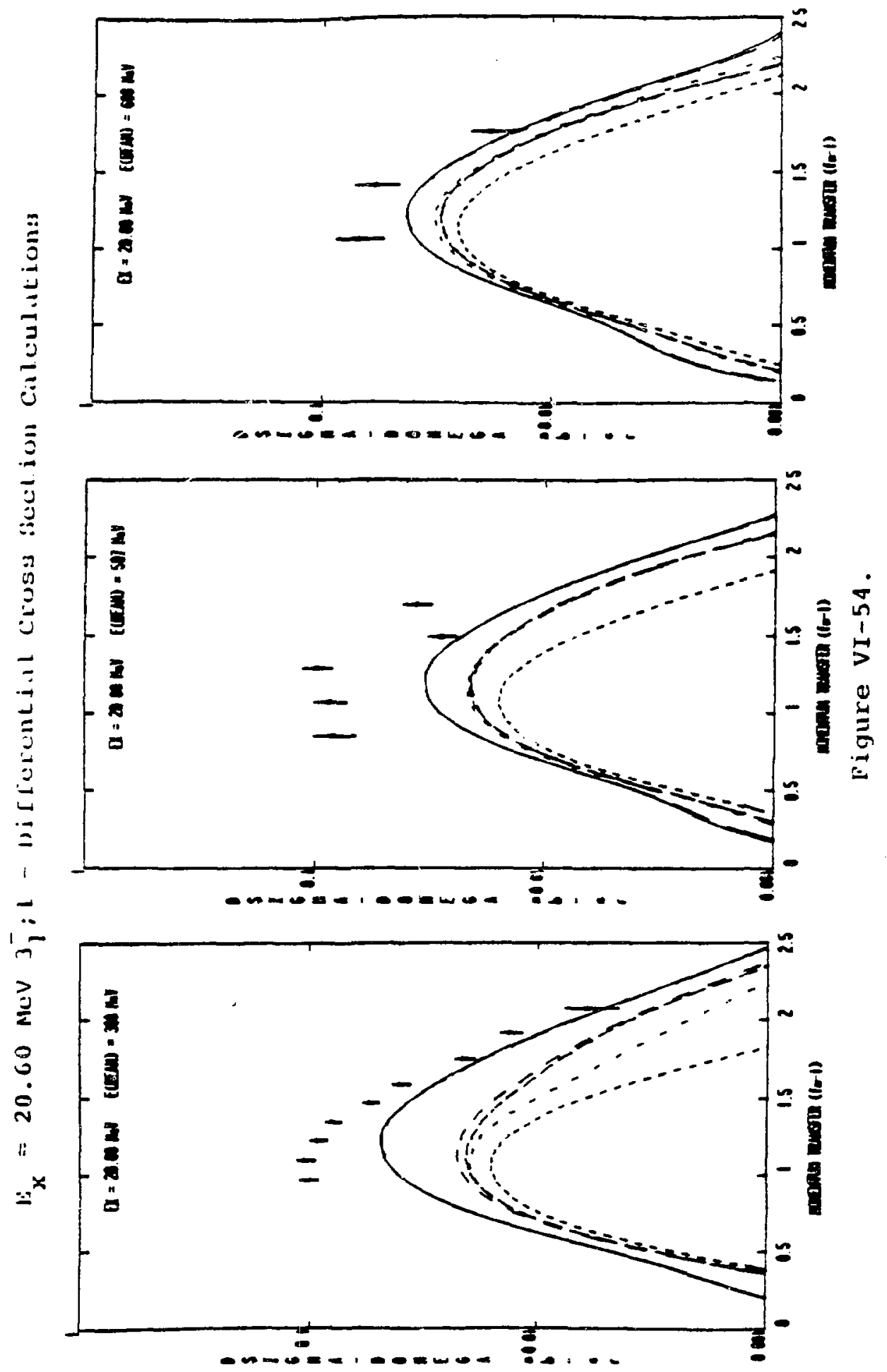


$E_{X}=20.60 \mathrm{MeV} 3_{1}^{-} ; 1$ - Analyzing Dower Calculations
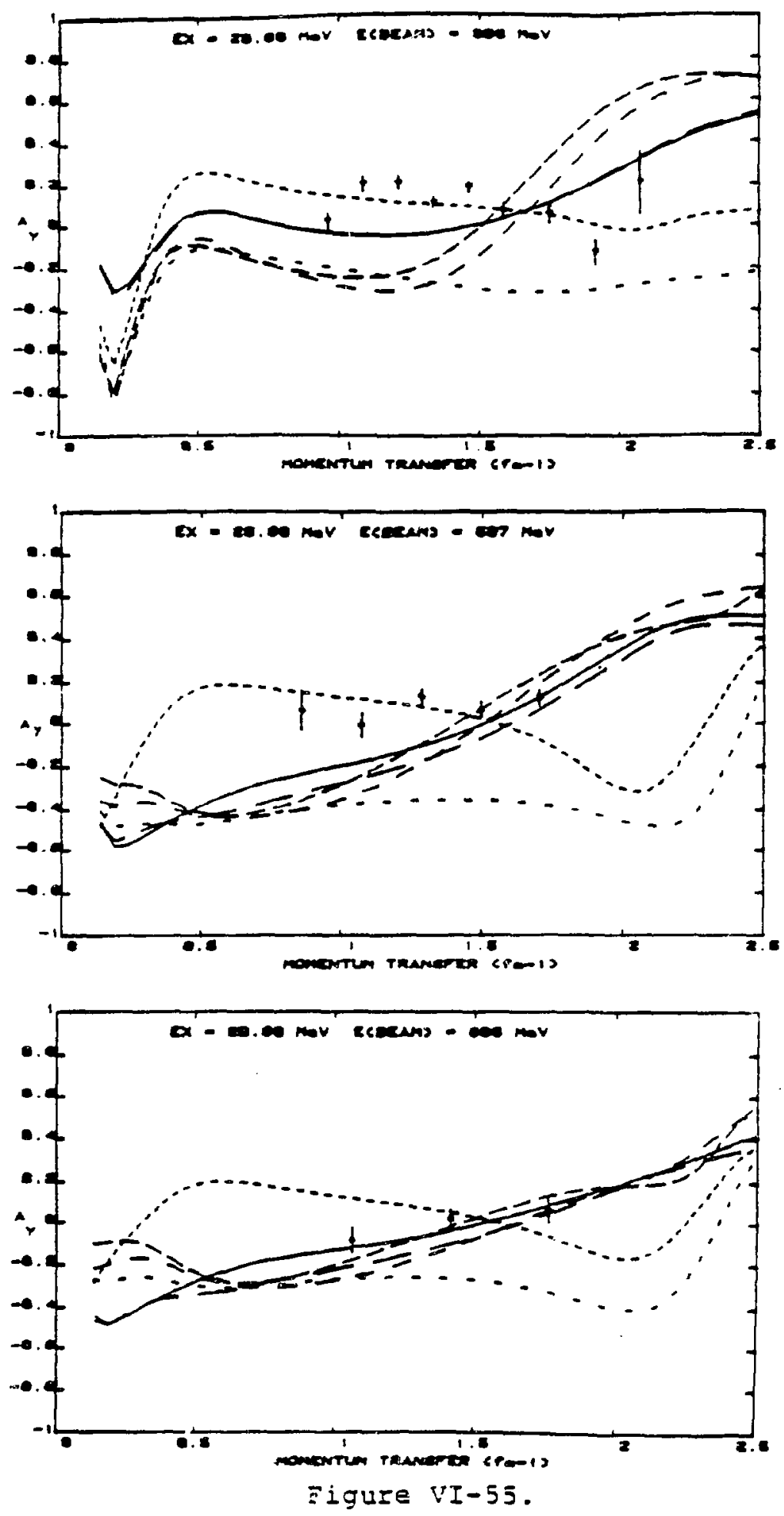
$E_{x}=20.60 \mathrm{lieV} 3_{1}^{-} ; 1$ - Comparative Calculations for Observables
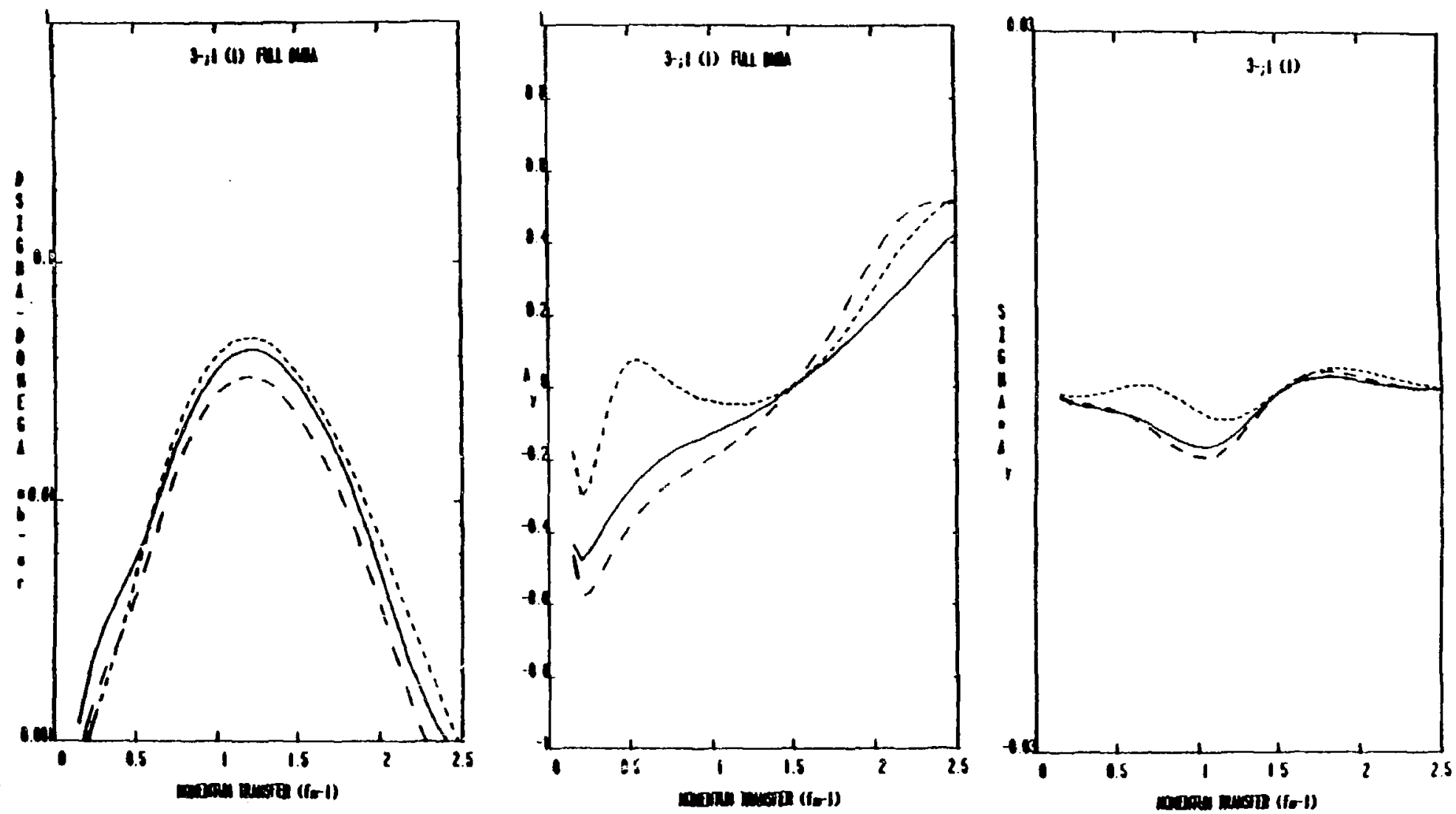

Figure VI-56. 
consistent with those for the $2^{+} ; 1$ state at 16.11 MeV. The shapes of both the differential cross section and the anaiyzing power data are reasonably well reproduced. The calculated cross sections required renormailization by a factor of about two at $398 \mathrm{MeV}$ to 1.4 at $698 \mathrm{MeV}$ to bring them into agreement with the data. The real direct central and real direct tensor terms contribute to the excitation of this state, and knock-on exchange reduces the purely direct cross sections by a factor of two, consistent with the $2^{+}$; state.

Comfort et. al. ${ }^{9)}$ assumed a pure $p_{3 / 2}$ to $d_{3 / 2}$ transition as had been done by Buenerd et. al. 60 ) In order to Eit the data at $200 \mathrm{MeV}$ the caiculated cross section had to be rescaled by a factor of 0.1. It is apparent that the assumption of such a pure configuration is not good, especialiy in the ilght of the transition densities derived by Millener. The $3^{+} ; 1$ calculation at $200 \mathrm{MeV}$ requires a renormalization of 0.6 , but does not reproduce the anaiyzing power data as well as the $3^{-} ; 1$ transition for large angles.

Therefore, based on the results of the calculations for this work and the work of others, we may conciude that: our data are consistent with an assignment of $3^{-} ; 1$ to this state, but the possibility of $3^{+} ; 1$ strength cannot be discoursted. 
Scale Factors for DWIA Computations

\begin{tabular}{|l|c|c|c|c|}
$J^{\pi} ; T$ & $E_{X}$ (MeV) & $398 \mathrm{MeV}$ & \multicolumn{1}{c}{$597 \mathrm{MeV}$} & $698 \mathrm{MeV}$ \\
\hline $2^{+} ; 0$ & 4.44 & 2.10 & $3.25(1.55)$ & $3.74(1.75)$ \\
$3^{-} ; 0$ & 9.64 & 1.85 & $2.93(1.58)$ & $3.00(1.62)$ \\
$1^{-} ; 0$ & 10.84 & - & 1.83 & - \\
$2^{+} ; 10$ & 16.11 & 0.54 & $0.83(1.54)$ & $0.67(1.24)$ \\
$2^{+} ; 1 \mathrm{NQ}$ & 16.11 & 0.83 & $1.56(1.88)$ & $1.32(1.59)$ \\
$1^{+} ; 0$ & 12.71 & 1.00 & $1.50(1.50)$ & $1.40(1.40)$ \\
$2^{-} ; 0$ & 18.30 & 1.50 & $1.60(1.05)$ & $1.50(1.00)$ \\
$4^{-} ; 0$ & 19.28 & 1.60 & $1.30(0.80)$ & $1.60(1.00)$ \\
$1^{+} ; 1$ & 15.11 & 1.00 & $1.50(1.50)$ & $1.40(1.40)$ \\
$2^{-} ; 1$ & 16.58 & 0.33 & $0.50(1.52)$ & $0.57(1.73)$ \\
$2^{-} ; 1$ & 19.40 & 0.33 & $0.33(1.00)$ & $0.33(1.00)$ \\
$4^{-} ; 1$ & 19.65 & 0.33 & $0.60(1.80)$ & $0.55(1.70)$ \\
\hline
\end{tabular}

Number in parentheses indicates scaling required to fit data after renomalizaton to $398 \mathrm{MeV}$ data.

Table VI-3. 
VII. CONCLUSIONS

Wo have studied the excitation of the ${ }^{12} \mathrm{C}$ nucleus through the medium of good resolution elasti: and lnelastic polarized protos scattering at 400, 600, and $790 \mathrm{MeV}$. The data presented in this work, togethes with other data at both lower and higher energles, provide a comprehensive excitation function for this nucleus over an incldent particle energy range of $800 \mathrm{MeV}$. This is the flrst systematle study of any nucieus over this energy range. No prevlous irelastic data exist at $700 \mathrm{MeV} ;$ the only previous data at 600 and $408 \mathrm{MeV}$ were a few studies of strong natural parity coliective states at 600 and $400 \mathrm{MeV}$ and the $1^{+}$states at 400 MeV, ail with unpolarized beam ${ }^{13,141}$.

The efflelency of the Mtcroprogramable Branch Driver capability to reject events by software, resulting in improved tapling efficlency for events of interest, was conciusively demonstrated. The increased data acquisition rate through the use of buffered electronics was most useful in compieting the data-taking in the allotted time.

A detalled study of the inelastic transitions served a variety of purposes. Systematic trends in the analyzing power data were shown to be a signature of the lsospin transfer for unnatural parity states. Speciflealiy, negative vaiues for low momentum transfers rising to posigive vaiues as q increages are strongly indicative of 1 soscaiar 
eransitions of unnaturai parity over the incident proton energy range studied in this work. This was ampiy iijustrated for the $1^{+} ; 0$ and $2^{-} ; 0$ states. Analyzing powers ciose to zero for low momentun transfer were shown to be consistent wth isovector states of both natural and unnatural parity. Spin-fI Ip measurements are nacessary to discriminate between natural and unnatural parity isovector states.

Perhaps the most interesting feature of the data themselves is the dependence on incident energy of the absoiute cross sections. The lndependence of the magnitude of the absoiute cross section as a function of energy has been shown to be indlcative of lsovector naturai partty and ail unnaturai parity transitlons. Monotonicaliy increasing cross sections are identifled with isoscalar natural parity states. The energy dependence of the naturai parity states refiects the increase in elastic scattering cross sections and the strong increase in proton-proton scattering cross section over this energy range ${ }^{31}$.

The phenomenologicai optical model was found to be reasonably aderuate in describing the elastic scattering differential cross sections. Reasonabie fits to the first minimum at 597 and $698 \mathrm{MeV}$ required the introduction of a surface term with a large radius. The fit to the 597 llev data also seems to require that the smail reai repulsive potential have an unphysicaliy iarge radius. Expected trends Eor the energy dependence of the strengths of the potentials 
were quaititativiy reproduced. Tho modei continues to show difficuity in reproducing the shape of the eiastic scattering analyzing power.

The effective nucieon-nucleon interaction of Love and Franey ${ }^{3)}$ has been examined in constderable detali. The Imaginary central scalar-isoscalar term dominates the excitation of natural partty isoscalar states. These states also require contributions from the real and imaginary isoscalar spin-orbit terms, especiajiy for large momentum transfer. Thie real isoscalar spin-orblt term aiso drives isoscaiar unnatural parity excitations for mediun so large momentum transfers... The tensor force ts the doninant component in the excttation of ali lsovector states; the exchange component being important also for isoscalar unnaturai parity states at small momentum transfer. The reai 1sovector tensor component dominates all classes of 1 sovector transitions, especiaily at large momentum transfer. The central isovector term contributes to the high momentum transfer axcltation of natural parity states, while the real spin-isospin flip central term drives the iow momentum transfer excltation of unnatural parity isovector states.

The central scalar-isoscalar term is the only one to exhibit strong energy dependence, and this is reproduced approximately by the 1 soscalar natural partity data. The less dramatic energy dependence of the tensor interaction and various weaker components of the centrai and spin-orbtt in- 
teractions responsible for other transitions show remarkable canceijations which permit the overail cross sections for these transitions to be almost energy independent whije the relative contributions of the individuai components are varying as a function of $q$ and $E_{p}$.

The anaiysis presented in Chapter VI allows us to draw some conclusions concerning how well the significant components of the interaction are determined. It must be polnted out that for most states severai components are required to furnish a satisfactory description, and this necessarily means that information about individual components is difficuit to extract.

We consider first the scalar-isoscalar central interaction $t_{0}^{c}$. The cross sections for naturai partty isoscaiar states increase at the maximum by 2.8 and 3.5 Erom 398 to $59 \overline{7}$ to $698 \mathrm{Mev}$. The magnitude of the ratio of the square of the scaiar-isoscaiar term for the 650 MeV force reiative to the $425 \mathrm{MeV}$ forca is 2.6. However, Tabie VI-2 shows that aciditional renormalizations of about 1.6 are required for calculations done with the 650 MeV force relative to the 425 MeV force to bring the caiculations into agreement with the data. Noting that inelastic electron seattering data requice an upward renormalization by a factor of two53,54), consistent with the $398 \mathrm{MeV}$ inelastlc proton scattering data, we may deduce that the strength of the scalarisoscalar term at $425 \mathrm{MeV}$ is essentlaliy correct, while that 
for $650 \mathrm{MeV}$ is too smail by a factor of 308 . This observation is consistent for ail naturai parity isoscaiar states analyzed.

Tive centrai spin- and isospin-flip term tor dominates the low momentum transfer excltation of isovector unnatural partty states. Electron scattering data have shown that the wavefunction of the 15.11 MeV state is well determined to a momentum teansfer of about $1.5 \mathrm{fm}^{-1}$ without renormalization 53,54 ). The excelient fit to the low $q$ data for this state indicates that this component is correct to within the uncertainties of the data, or 168 , at 425 Mev, but the cenormalization of 1.5 required for the 656 MeV calculations indicates that this component is 308 too low for this force. The magnitude of this term in the Love-praney interaction shows a silght decrease from 425 to $650 \mathrm{MeV}$ which cannot be determined to within the error bars on the data.

The scalar spin-orbit component is predicted to be Independent of energy and to be important for large momentum transfer. This is sien as an enhancement in the calculated cross sections for the natural parity isoscalar states at Iarge $q$, more so for the caiculations for the $650 \mathrm{MeV}$ force than those for the $425 \mathrm{MeV}$ force. ThIs term dominates the excitation of the $2^{-} ; 0$ state at $18.30 \mathrm{MeV}$, and aiso the tentatively tdenttfled $4^{-}, 0$ state at $19.28 \mathrm{MaV}$. The spin-orblt force adequately describes the data without enhancements due 
to the tensor force, aithough the evidence is somewhat weaker for the $4^{-} ; 0$ state. Since eiectron seattering renormailzation factors are not availabie, we cannot make a definite starement about the magnitude of this component, other than It may be correct to within the factor required to renormajize the calcuiations, or 30t. However, the energy independence (taking both real and imaginary parts) is cieariy evident. This term is also primarily involved in the medium momentum transfer excitation of the $1^{+} ; 0$ state at $12.71 \mathrm{MeV}$. Comparisons with the data here affirm the Independence with energy and indicate that the magnitude may be correct to within 20 in this region. The smail momentum transfer strength is considerabiy reduced for the $650 \mathrm{MeV}$ force, and significantiy worsens the overail fit to the data.

The isoscalar tensor term, $t_{0}^{T}$, contributes oniy to the smaij momentum transfer dependence of the $i^{+} ; 0$ state at 12.71 MeV, The Love-Franey force predtcts no discernible energy dependence for this term. We find the relative enhancement above the underiying spin- orbit contributions to be roughly constant for both the 425 and $650 \mathrm{MeV}$ forces. The Elt to the $398 \mathrm{MeV}$ data shows this term to be accurate to about 15 for the $425 \mathrm{MeV}$ force. The flts to the 597 and 698 MeV data indicate that this term may be no more accurate than 388, and Iow. However, caution is advised because of the previousiy atscussed problems with the spin=orblt force.

The last significant term is the isovector tensor 
component, which dominates the excltation of several states at iarge momentum transfer. The medium momentum transfer contributions to tha cross section for the 15.11 MeV state indicate a reasinable accuracy of about 26 given the validity of the wave functions in thls region of g, the strength generaliy being low wh th respect to the data. It is not posstbie to make any statement for largo momentum transfers because of structure uncertainties. The $22^{-}: 1$ state at 16.58 MeV agrees with electron scattering results which overestimate the data by a factor of. $1.5^{54}$, with the overestimation increasing monotoilically from 800 MeV to lower beam energies. For the 650 MeV fora this term is exclusively responsible for the strength, whereas the observed strength at $425 \mathrm{MeV}$ is predicted solely by the tcterm, with the tensor component serving only to overestimate the data. Renormalization consistent with the electron scattering factor of 1.5 downward indicates that the serength of this term at 650 MeV may be low by about 15*. The strong contribution of tot at 425 MeV prociudes extracting a factor at that erergy. The $2^{+} ; 1$ state at $16.11 \mathrm{MeV}$, having been renormalized to electron scattering data 53,54 ), requires an enhancement by a factor of 1.6 for agreement between the 650 MeV force and 597 MeV data. This lmpiles that the tensor part of the force being considered here is about 38 low ar 597 MeV. The $4^{-} ; 1$ state at $19.65 \mathrm{MeV}$ exhlbits trends ciosely similar to those of the $16.58 \mathrm{MeV}$ state, but the absence of an electron scattering renormadization factor precludes further 
analysis.

None of the other terms in the interaction contribute with sufficient strength to any state to warrant comment.

The preceding conclusions have centered around the magnitudes of the cross sections. The analyzing powers are particularly sensitive to interference between contributions from different parts of the force. The quality of fits to the analyzing powers varled widely, some beling very good (e.g. $12.71 \mathrm{MeV}$ state for $398 \mathrm{MeV}$ ) and some very poor (e.g. $16.11 \mathrm{MeV}$ state at large q for all energies). Statenents regarding the relative importance of different terms in the interaction are uncertain because of lack of knowledge of the phases involved, and must be treated with caution.

Fits to the anaiyzing powers which provide information about the Love-Franey force are as follows. Isocaiar natural parity states are reasonably weil reproduced to within a phase. The combination of spin-orbit distortions and the spin-orbit component of the interaction resuits in approximatoly the correct magnitude for these analyzing powers. This is somewhat surprising since the cross section evidence points strongly to a spln-orbit force that is too weak at $650 \mathrm{MeV}$. This suggests a reasonably satisfactory phase between central and spin-orblt terms. The success achieved in fitting the analyzing powers of the lsoscalar 
unnaturai partty states $1^{+} ; \theta$ and $2^{-} ; 0$ at $398 \mathrm{MeV}$ strongiy suggests that the relative phase of the spin-orbit and imaginary isoscalar tensor components is reasonabiy weil determined. The very smali anaiyzing powers measured for smail $q$ in lsovector naturai and unnaturaj partey states points to the correctness of the weak isovector spin-orblt component. The iarge monentum transfer dependence of the $4^{-}$; 1 state is aiso reasonabiy weli reproduced.

The inability of the saiculations to reproduce the 12.71 and $18.30 \mathrm{MeV}$ data for the $550 \mathrm{MeV}$ force is a strong Indication of a deficiency in the lsoscalar spin-ortit force, which supports assertions from cross section comparisons. Furthermore, the difference in sign for predicted and measured high monentum transfer analyzlng powerg for the isovector $2^{+}$state at $16.11 \mathrm{MeV}$, together with the phase probiems apparent for large q for the 15.11 MeV state Ind 1cate that the relative phase of the spin-orbit and real isovector tensor terms is not weil determined. This is also true for the $2^{-}$; 1 state at $16.58 \mathrm{MeV}$.

Overail we find the strengths of the dominant terms in the $425 \mathrm{MeV}$ force to be qualitatively and quantitatively weil determined. Such a strong statement of agreement cannot be made for the 650 MeV Eorce, where a systeratic lack of strength of the order of 20 to 30 is found for the doninant components of the interaction, esperialiy the scalarisoscalar central term and the lsoscalar spln-orblt term. 
Nevertheiass, the Love-Franey parameterization has been demonstrated to be a useful dascription for the study of inelastic proton seattering, and with further scudy and refinement may contribute significantly to the understanding of the systematics of intermediate energy proton-nueleus scattering.

The effects of knock-on exchange were found to be important. Exchange was found to reduce the calculated cross sections from purely direct values by a factor of 3.5 for isoscalar natural parity states. A reduction by a factor of 2 was noted for isovector naturai parity states as weil as for the better known unnatural parity transitions. Very smaji effects were observed for seiect states in the high excitation region.

The satisfactory performance of the Love-Franey interaction in describing the more weli known states permitted the use of calculations to aid in the determination of the spins and partites of levels in the excitation region 18 to $21 \mathrm{MeV}$. We were able, on the basls of systematics of the data and the results of the DWIA calculations, to make suggested assignments of spin, parity and isospin to states in this region as Iisted in Table VII-1. These assignments are consistent with tentative plon scattering assignments ${ }^{26}$ ) for the $2^{-}$and $4^{-}$states, but they are different from tentative ineiastic eiectron seattering assignments ${ }^{24)}$. 


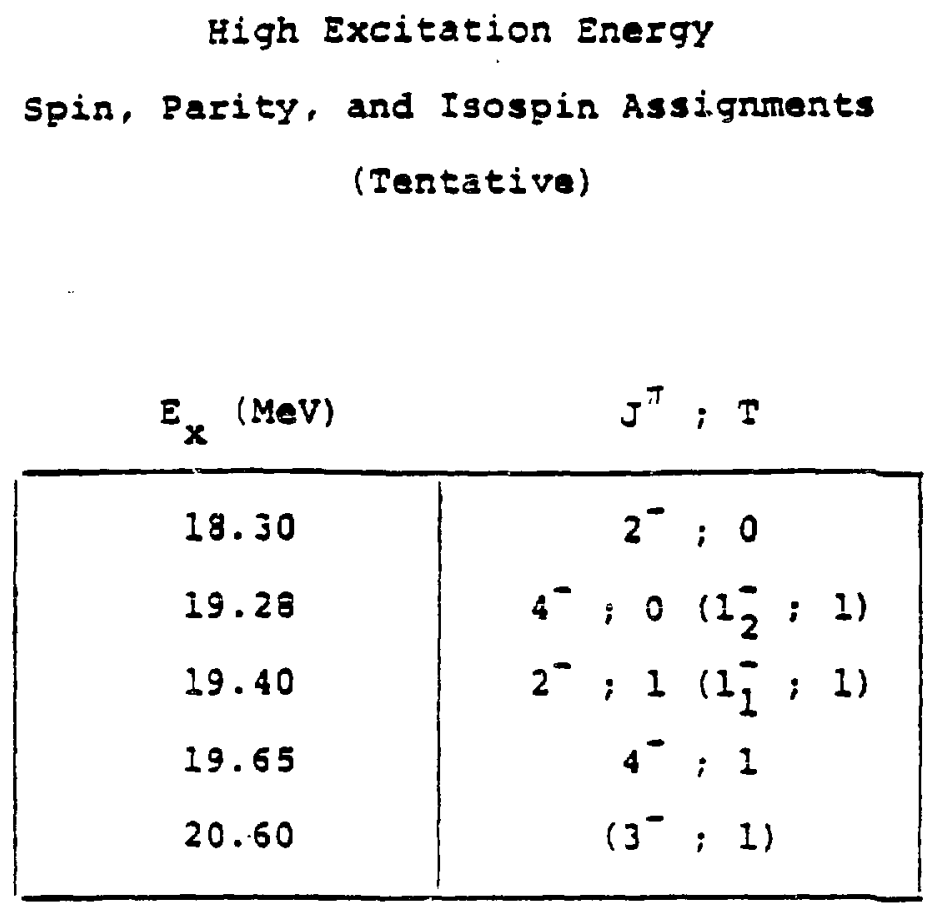

Table VII-1. 


\section{ACXNOWLEDGEMENTS}

I am deeply indebted to my advisor, professor Charles Giashausser, for his patience and encouragement during the four years I have worked with him. For the free hand he has given me. for his continuing concern for the welfare of both myself and my family and for giving me an insight into what is meaningful, I am most grateful. I wish to also thank many faculty members at Ruegers for many hour 3 of stimulating discussion, especially professors Noemie Kolier, Peter [indenfeld, Joel Shaptro and Ailen Robbins. I acknowledge professor Georges Temmer for his accompilshed ieadershtp of the Rutgers Nuciear Physics Laboratory.

I wouid ilke to thank Dr. Robert Kaita and Mr. James Hutd EOt vaiuabie iessons patientiy taught to me during my eariy days at Rutgers. I aiso acknowiedge Dr. Bo shu for doing such a fine job of maintaining the computer systems, without which this work wouid have been much more difeicuit. I owe much to professor Felix Sannes for the use of computing facilities and to Dr. Joseph comfort for aid with the code DW81, without whith the calcuiations presented in this work would not have been possible. I wish to acknowiedge the willing assistance of Jeffrey Woilinkl during the more tedious aspects of the data anaiysis and reduction.

The tanden matntenance staff at Rutgers, especiajiy Richard Leidich, must be thanked for their assistance and 
support over the years. To those who foilow, especiaily Sirish Nanda, Chuck Meitzier, Curtis Beil, Doug Baijon and Tom gright, many thanks for your assistance and friendship.

I am deepiy indebted to many at LAMPE for thels assistance and hospltality during my many sojourns in los Alamos. Thanks are due to Drs. James Amann, Rlchard Boudrte, Thomas Carey and Susan Seestrom-Morrts for many patient, helpful and often repeated discussions. I am especlaily indebted to Dr. John McClelland for his encouragement, hospitality and efforts in my hetsif.

I en very gratefui to Mrg. Ruth Bennet and especialiy Mrs. Midied Aithouse for their kindness and continual concern for my weit-being.

Last, but most important, I wish to ackrowiedge the support, patience and understanding of my wife bindi, whose fortitude during some rather difficult times over the iast two years has aiways been remarkabie. I hope that she wili reap more than adequate benefits for her faith in me. 


\section{REFERENCES}

I. R. J. Glauber, article in "Lectures in Theoretical physics, Vol. I" (Interscience, New York) 1959

2. A. K. Kerman, H. Mckanus and R. M. Thaler, Annals of Physics 8, 551 (1959)

3. W. G. Love and M. A. Eraney, Phys. Rev. C24, 1073 (1981)

4. E. Ajzonberg-Selove, Nucl. Phys. A248, I (1975)

5. J. R. Comfost et. al., Phys. Rev. C21, 2147 (1980)

6. J. R. Comfort et. al., Phys. Rev. C23, 1858 (1981)

7. J. R. Comfort et. al., Phys. Rev. C24, 1834 (195i)

8. J. R. Comfort and W. G. Love, Phys. Rev. Lett. 44. 1656 (1980)

9. J. R. Comfort, to be published.

10. D. Hasselgren, P. U. Renberg, O. Sindberg and G. Tiball, Nuel. Phys. 69, 81 (1965)

11. A. Ingemarsson, 0 . Jonsson and A. Eallgren, Nucl. Phys. A319, 377 (1979)

12. G. S. Blanpied er. al., Phys. Rev. C18, 1436 (1978)

13. J.-t. Escudié et. al, Phys. Rev. C24, 792 (1981)

14. G. Bruge et. al., Jour. de Physique 40, 635 (1979)

15. M. Haji-Saeid et. al., Phys. Rev. Lett. 45, 880 (1980)

16. M. Baji-Saeid et. 1ㅡ., Phys. Rev. C25, 3035 (1982)

17. S. Coher and D. Kurath, Nuel. Phys. 73, 1 (1965)

18. D. J. Millener, private communication.

19. J. M. Moss et. âl., Phys. Rev. Lett. 44, 1189 (1980)

20. E. E. Sapirgtein, S. V. Tolokonnikov and S. A. Fayans, JETP Lete., 25, 513 (1977)

21. S. A. Fayans, E. E. Sapizstein and S. V. Tolokonnikov, Nucl. Phys. A326, 463 (1979) and Ehys. Lett. 923, 33 (1980)

22. H. Toki and w. Weise, Phys. Rev. Lett. 42, 1034 (1979)

23. J. Delorme, M. Ericson, A. Figureay and N. Giraud, Phys. Lett. 89B, 327 (1980)

24. J. B. Flanz et. al., Workshop on Nuclear Structure with Intermediate Eñergy probes, LANL ?ublication IA-8303-C, P. 418 (1980)

25. H. A. Thieisser, Nuel. Phys. 1335, 329 (1980) 
26. C. I. Morris, Workshop on Nuclear Structure with Intermediate Energy Probes, LANL Publication LA-8303-C, p. 57 (1980)

27. B. C. Karg, Ph. D. Thesis, University of Pittsburgh, (1982) (Unpublished)

28. C. J. Batty, Nucl. Phys. 23, 562 (1961)

29. H. Palevsky et. al., Phys. Rev. Lett. 18, 1200 (1967)

30. D. F. Jackson, Nuclear Reactions (Methuen) (1970) and references therein.

31. M. H. MacGregor, M. J. Maravcsik and H. P. Stapp, Ann. Rev. of Nucl. Sci. 10, 291 (1960)

32. D. V. Bugg et. al., Phys. Rev. C21, 1004 (1980)

33. R. A. Arndt, private communication.

34. M. H. MacGregor, R. A. Arndt and R. M. Wright, Phys. Rev. 182, 1714 (1969)

35. E. Petrovich, H. McManus, V. A. Madsen and J. Atkinson, Phys. Rev. Lett. 22, 895 (1969)

36. W. G. Love, Nucl. Phys. A312, 160 (1978)

37. J. Raynal, Trieste Lectures in Nuclear Theory (1971)

38. B. Zeidman, LASI Report LA-4773-MS, Part I, (1971)

39. G. S. Blanpied, Ph. D. Thesis, LASL Thesis LA-7262-T, (1978)

40. I. G. Atencio, J. F. Amann, R. L. Boudrie and C. I. $\because o=$ is, Nucl. Inst. and Meth. 187, 381 (1981)

41. S. Shlaer, unpublished.

42. R. Ridge, unpublished.

43. S. J. Seestrom-Morris et. al., private communication.

44. C. M. Lederer and V. S. Shirley, Table of Isotopes, 7 th Ed., (Wiley) (1978)

45. R. P. Liljestrand, Ph. D. Thesis, University of Texas, (1978) and Phys. Rev. Lett. 42,363 (1979)

46. J. R. Comfort, unpublished.

47. W. G. Love, private communication.

48. R. Schaeffer and J. Raynal, unpublished.

49. J. R. Comfort and W. G. Love, unpublished.

50. I. Ray, Phys. Rev. C20, 1857 (1579)

51. J. Kelly et. al., Phys. Rev. Lett. 45, 2012 (1980)

52. J. R. Comfort et. al., Phys. Rev. C24, 1844 (1981)

53. J. B. Flanz, Phys. Rev. Lett. 41, 1642 (1978) and references therein. 
54. J. B. Flanz, Ph. D. Thesis, University of Massachusets, (1979) (Unpublished)

55. A. Friebel et. al., Nucl. Phys. A294, 129 (1978)

56. T.-S. Lee and D. Kurath, Phys. Rev. C21, 293 (1980)

57. F. G. Adelberger et. al., Phys. Rev. C15, 484 (1977) and references therein.

58. C. L. Morris et. al., Phys. Lett. 998, 387 (1981)

59. J. Dubach and W. S. Haxton, Phys. Rev. Lett. 41, 1453 (1978)

60. M. Buenerd et. al., Nucl. Phys. A236, 377 (1977)

61. D. J. Millener and D. Kurath, Nucl. Phys. A255, 377 (1978)

62. J. R. Comfort, private communication.

63. D. J. Millener, private communication.

64. M. Buenerd, Ph. D. Thesis, Grenoble, (19/5) (Unpublished) 
APPENDIX

Part I

Sample MBD Cut File used in conjunction with the Q Analyzer HRSBUF for Software Event Rejection in the Microprogramable Branch Driver

Count 10

Sum 11

Reject Outside 107,360

Part II

Sample ALITST File used in conjunction with the $Q$ Analyzer HRSBUF for replay event analysis 


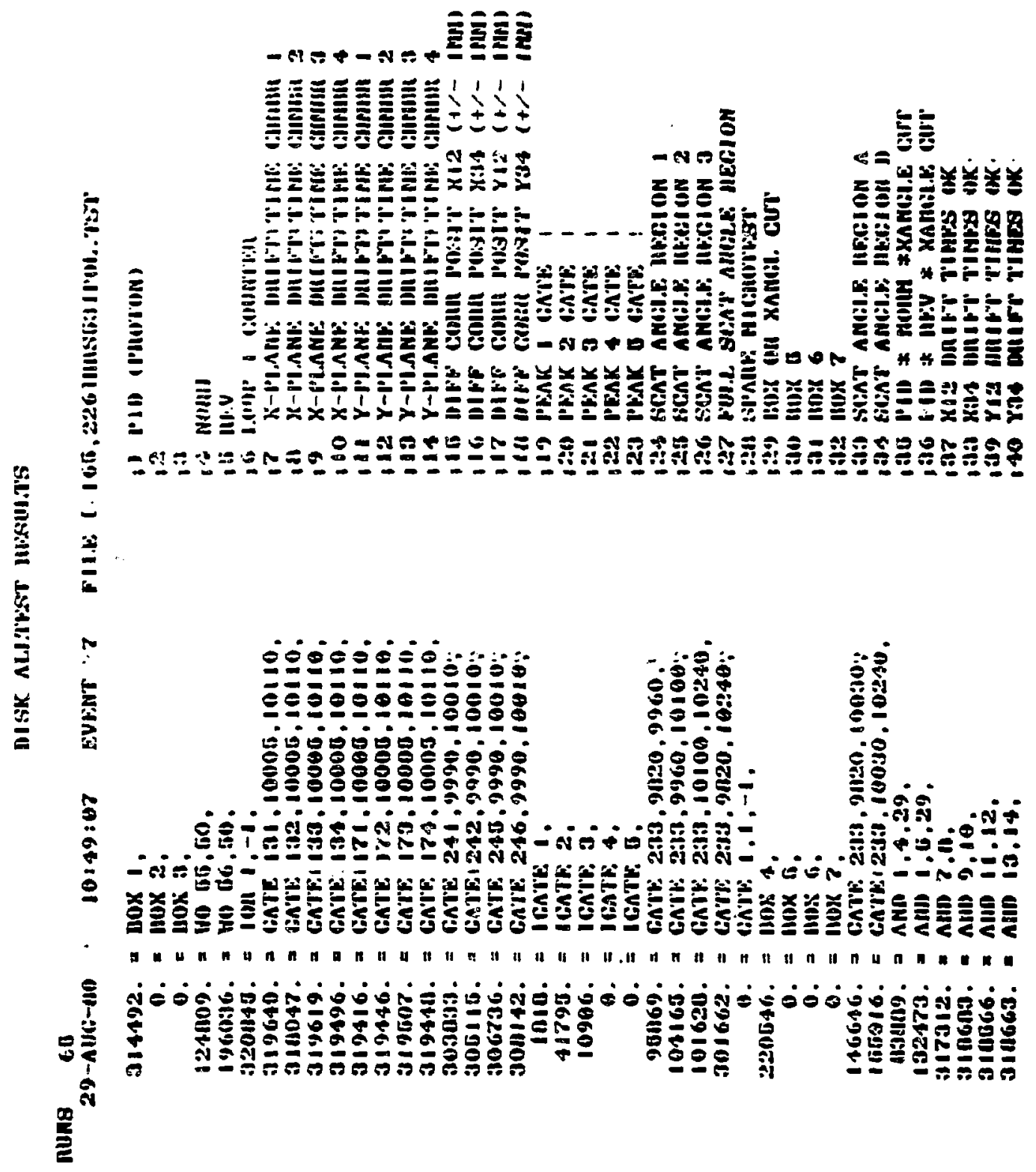



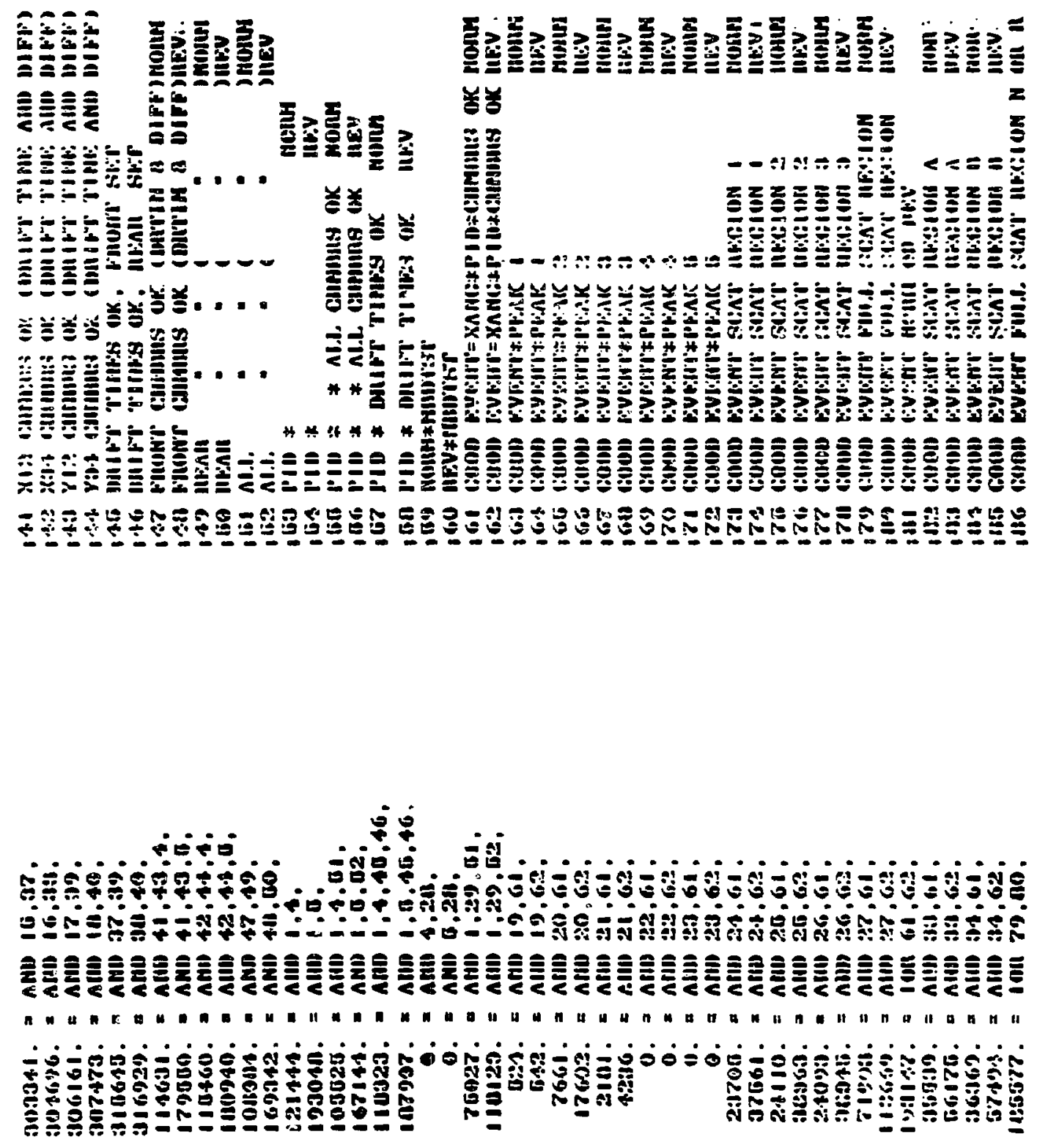
$61366 .=141173.74$.

lizt7al = INII 711.76.

G:1111. = 1011 77.711

$91714 .=1010$, Bi,

Diffil = 104 Ut, 10

$9208: 16 .=1011,-1$

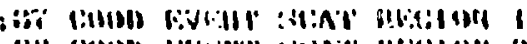

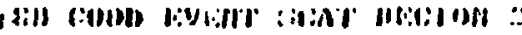

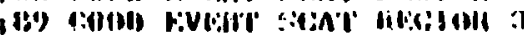

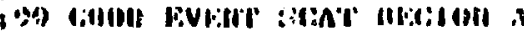

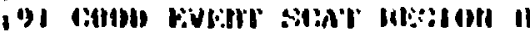

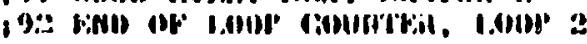

II III II

iII 11

in (HII

II III II

(III) II

box curs foll EVEurr 7

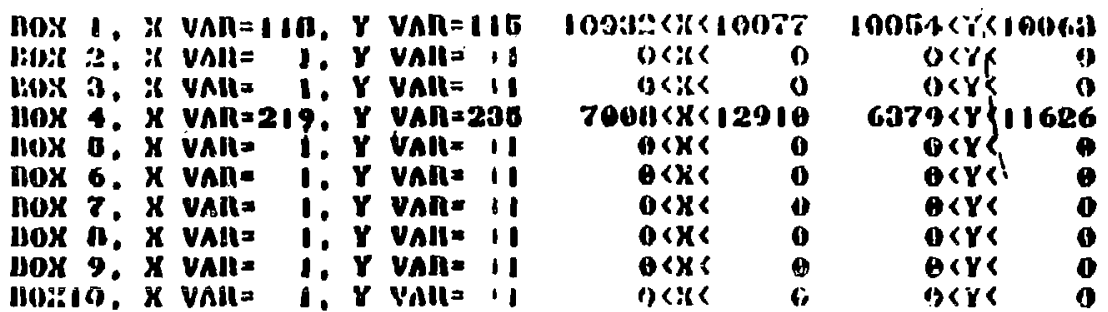

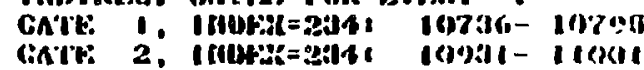

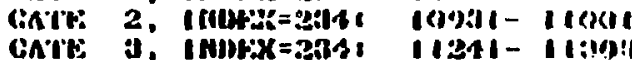


Sample Histtogram definition file used in conjunction with the $Q$ Analyzer HRSBUF for replay histogramming of the data

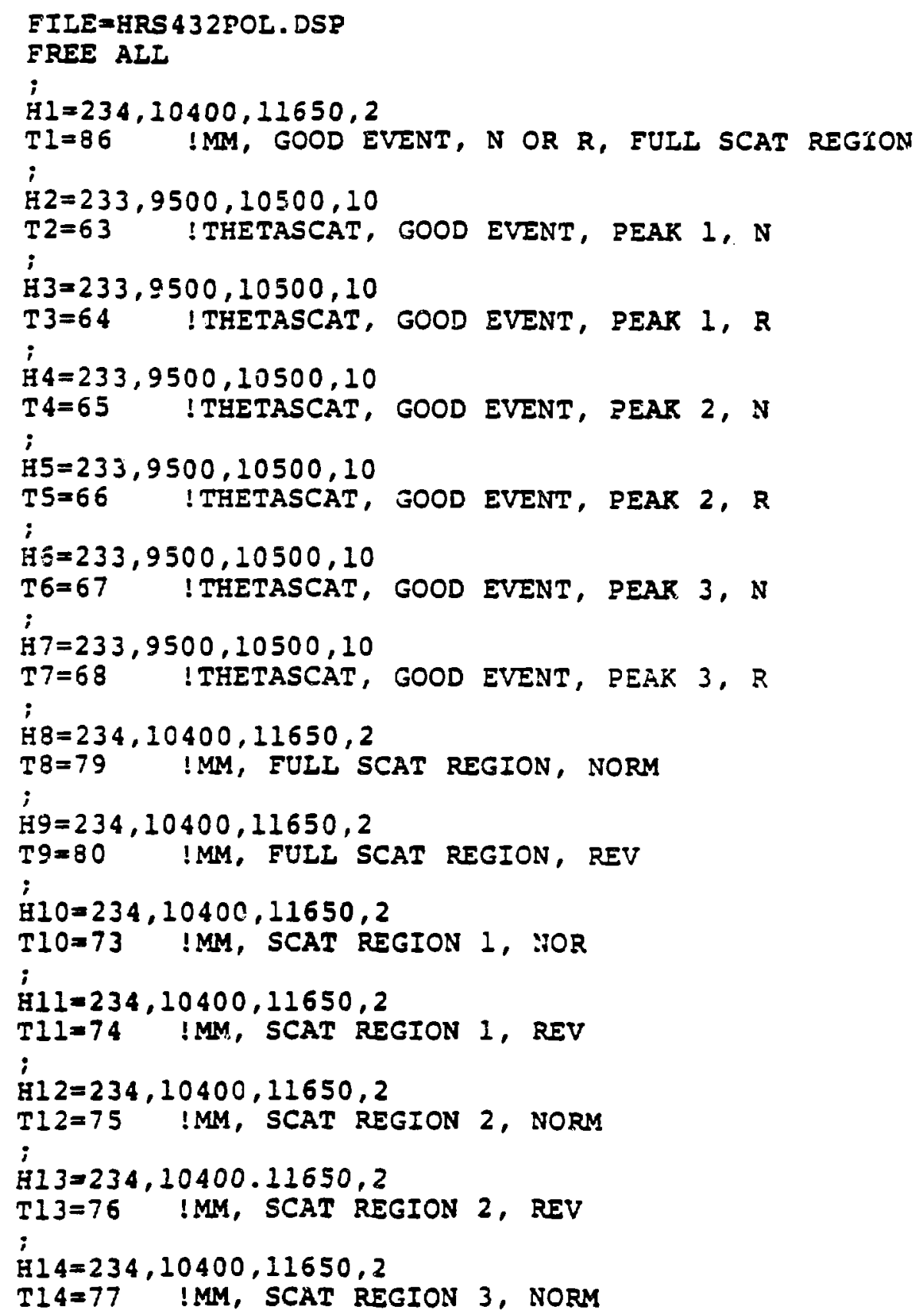




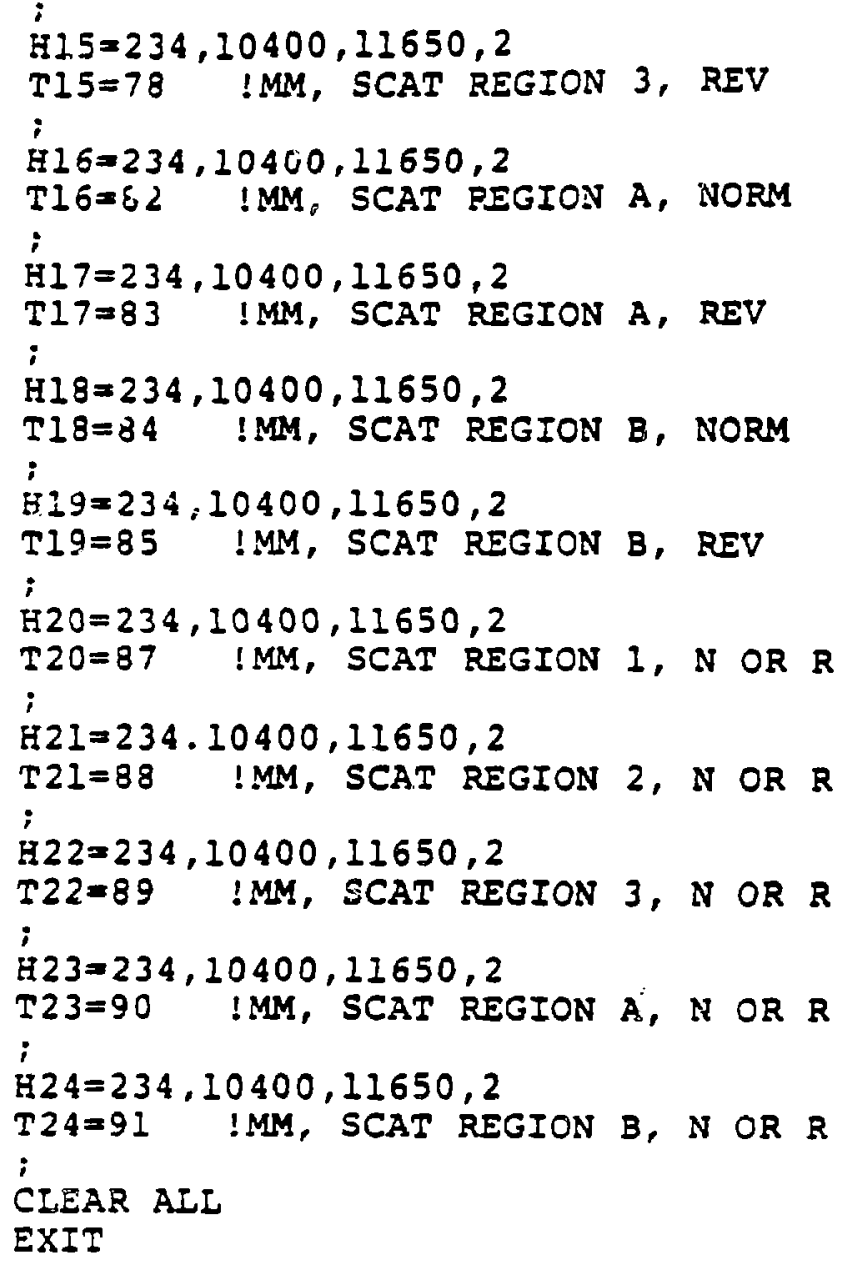

Tables of Experimental Differential Cross Sections and Analyzing Powers, Tables AI-A3. 
Experimental Argular Distributions

$$
{ }^{12} \mathrm{C}\left(\vec{p}, p^{\prime}\right)^{12} C^{*}-398 \mathrm{MeV}
$$

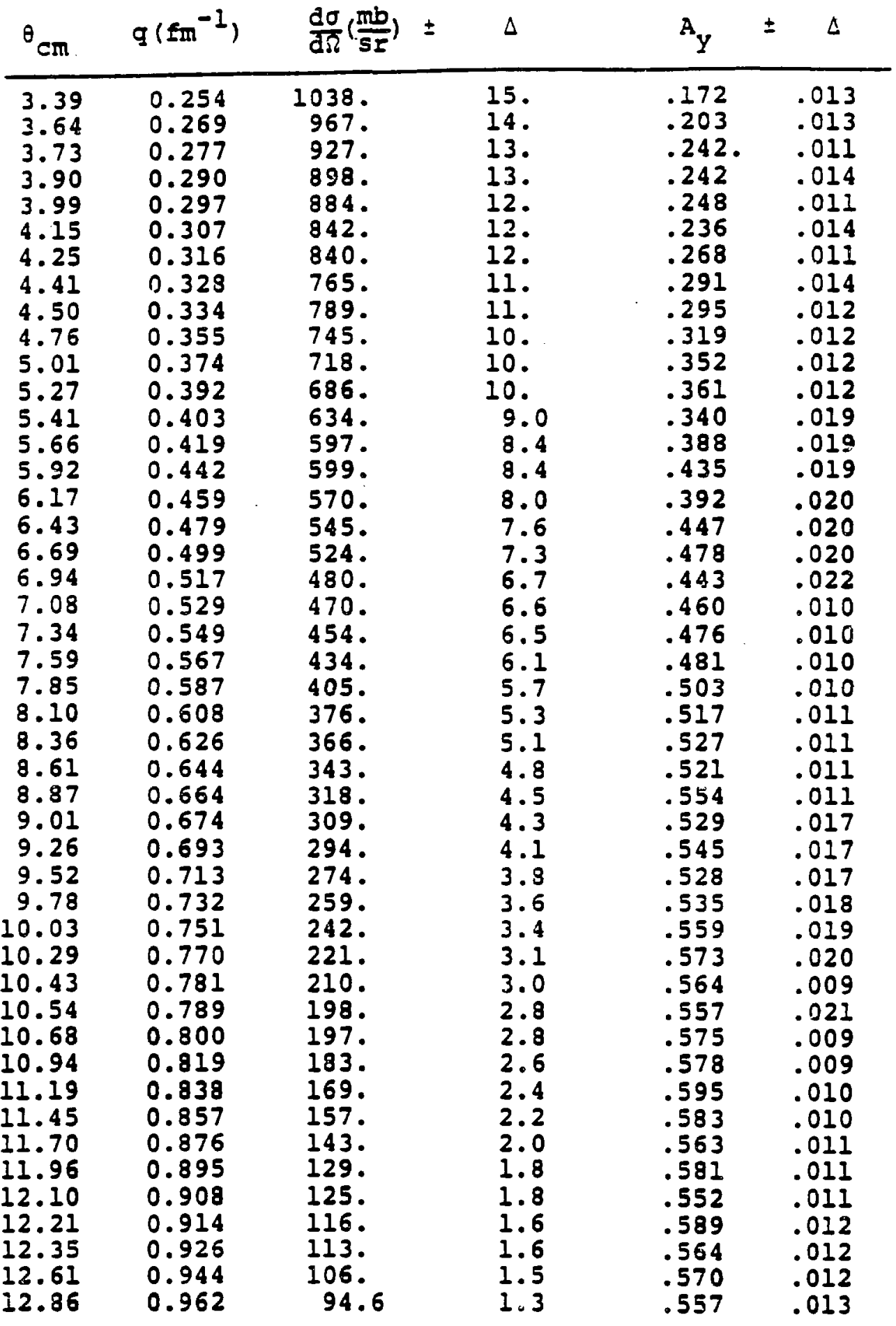




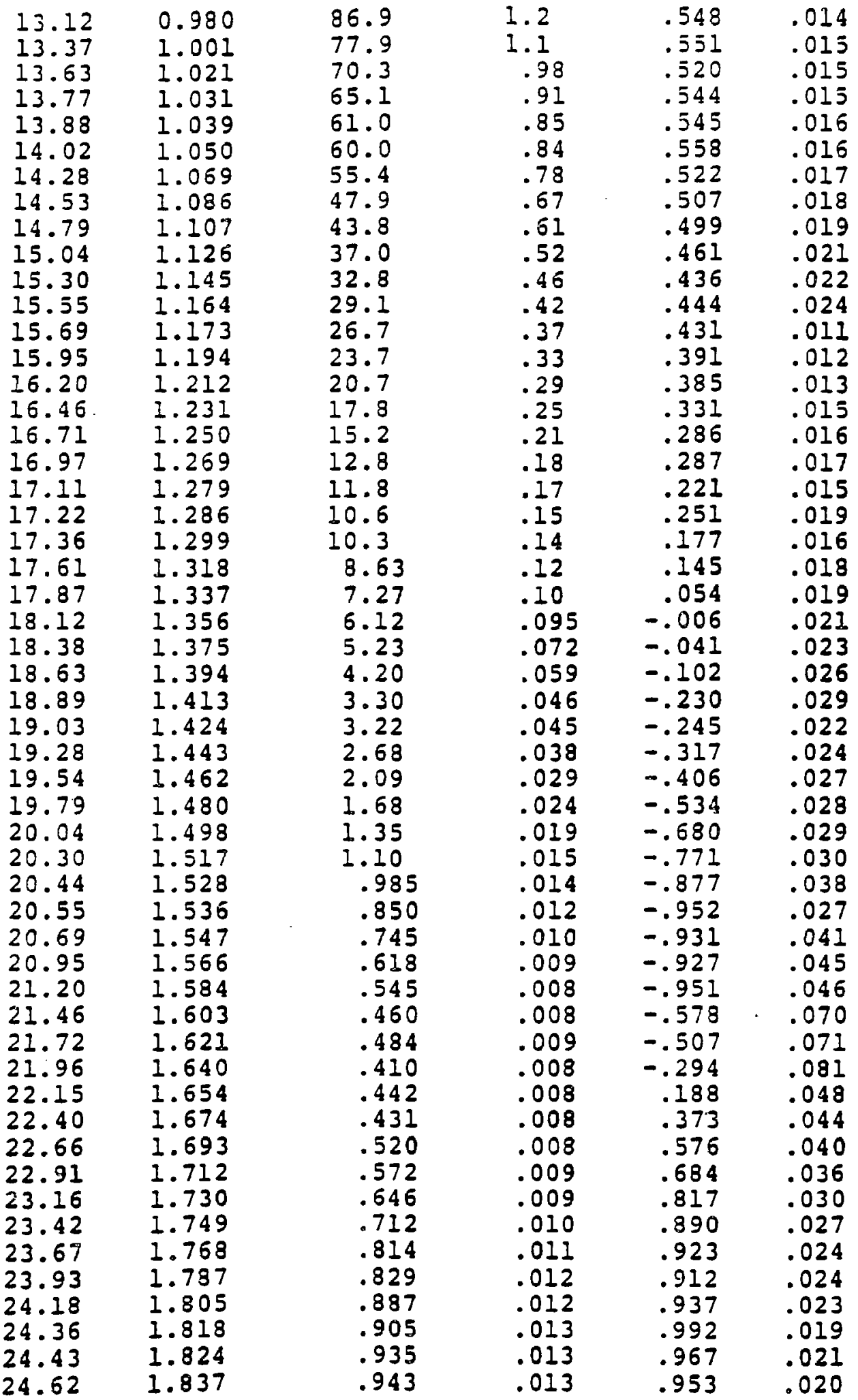




$\begin{array}{llllll}24.87 & 1.856 & .972 & .016 & .985 & .019 \\ 25.13 & 1.875 & 1.05 & .015 & .947 & .020 \\ 25.38 & 1.893 & 1.09 & .015 & .939 & .020 \\ 25.63 & 1.913 & 1.13 & .016 & .912 & .020 \\ 25.89 & 1.931 & 1.10 & .015 & .926 & .020 \\ 26.14 & 1.950 & 1.12 & .016 & .887 & .020 \\ 26.37 & 1.968 & 1.09 & .015 & .874 & .020 \\ 26.58 & 1.981 & 1.24 & .017 & .826 & .014 \\ 26.65 & 1.986 & 1.11 & .015 & .833 & .021 \\ 26.83 & 2.000 & 1.23 & .017 & .806 & .014 \\ 27.08 & 2.019 & 1.23 & .017 & .791 & .014 \\ 27.34 & 2.037 & 1.17 & .016 & .784 & .014 \\ 27.59 & 2.056 & 1.18 & .016 & .764 & .015 \\ 27.84 & 2.075 & 1.18 & .016 & .725 & .015 \\ 28.10 & 2.094 & 1.18 & .016 & .704 & .015 \\ 28.35 & 2.112 & 1.15 & .016 & .683 & .016 \\ 28.60 & 2.131 & 1.11 & .016 & .671 & .017 \\ 28.86 & 2.150 & 1.07 & .015 & .647 & .017\end{array}$

$$
2^{+}: 0-4.44 \mathrm{MeV}
$$

\begin{tabular}{|c|c|c|c|c|c|}
\hline $\begin{array}{l}7.0 .8 \\
7.34 \\
7.59 \\
7.85 \\
8.10 \\
8.36 \\
8.61 \\
8.87 \\
9.01 \\
9.26 \\
9.52 \\
9.78 \\
0.0 .3 \\
0.29 \\
0.43 \\
0.54 \\
0.68 \\
0.94 \\
1.19 \\
1.95\end{array}$ & $\begin{array}{l}0.529 \\
0.549 \\
0.567 \\
0.587 \\
0.608 \\
0.626 \\
0.644 \\
0.664 \\
0.674 \\
0.693 \\
0.713 \\
0.732 \\
0.751 \\
0.770 \\
0.781 \\
0.789 \\
0.800 \\
0.819 \\
0.838 \\
0.857 \\
0.876 \\
0.895 \\
0.908 \\
0.914 \\
0.926 \\
0.944 \\
0.962 \\
0.980 \\
1.001 \\
1.021 \\
1.031\end{array}$ & $\begin{array}{l}3.14 \\
2.36 \\
3.32 \\
3.77 \\
3.50 \\
4.39 \\
4.20 \\
4.61 \\
3.97 \\
5.35 \\
6.01 \\
5.55 \\
6.34 \\
7.53 \\
7.32 \\
6.34 \\
7.86 \\
8.04 \\
8.17 \\
7.68 \\
8.60 \\
8.61 \\
7.67 \\
8.32 \\
8.69 \\
9.22 \\
8.81 \\
9.29 \\
9.86 \\
10.2 \\
10.2\end{array}$ & $\begin{array}{l}.40 \\
.28 \\
.40 \\
.45 \\
.42 \\
.52 \\
.42 \\
.46 \\
.40 \\
.54 \\
.55 \\
.51 \\
.55 \\
.60 \\
.55 \\
.50 \\
.45 \\
.65 \\
.65 \\
.60 \\
.60 \\
.60 \\
.50 \\
.50 \\
.50 \\
.55 \\
.55 \\
.55 \\
.55 \\
.60 \\
.60\end{array}$ & $\begin{array}{l}.621 \\
.647 \\
.629 \\
.613 \\
.667 \\
.635 \\
.669 \\
.679 \\
.637 \\
.644 \\
.691 \\
.650 \\
.663 \\
.665 \\
.691 \\
.688 \\
.708\end{array}$ & $\begin{array}{l} \\
.030 \\
.050 \\
.035 \\
.029 \\
.027 \\
.027 \\
.026 \\
.026 \\
.020 \\
.026 \\
.022 \\
.018 \\
.018 \\
.018 \\
.018 \\
.017 \\
.035\end{array}$ \\
\hline
\end{tabular}




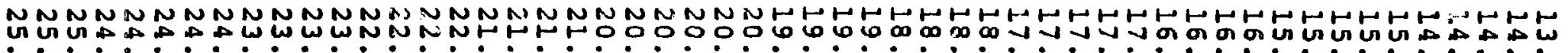
ow

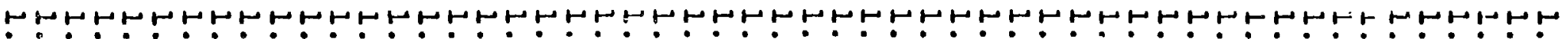
م 네

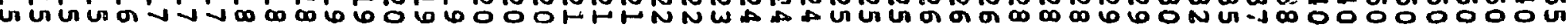

111

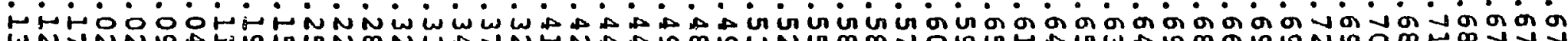

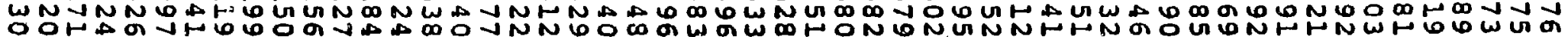

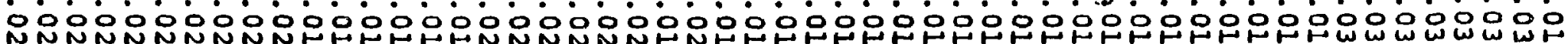

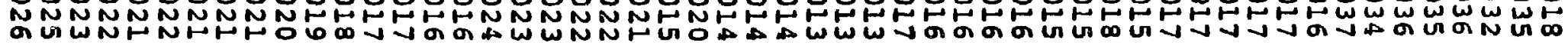




$\begin{array}{llllll}25.89 & 1.931 & 1.22 & .15 & -.183 & .027 \\ 26.14 & 1.950 & 1.09 & .14 & -.205 & .028 \\ 26.47 & 1.968 & .991 & .14 & -.238 & .029 \\ 26.58 & 1.981 & .910 & .12 & -.255 & .021 \\ 26.65 & 1.986 & .851 & .11 & -.294 & .031 \\ 26.83 & 2.000 & .833 & .10 & -.292 & .022 \\ 27.08 & 2.019 & .760 & .09 & -.352 & .022 \\ 27.34 & 2.037 & .671 & .08 & -.391 & .024 \\ 27.59 & 2.056 & .617 & .07 & -.429 & .024 \\ 27.84 & 2.075 & .557 & .07 & -.462 & .025 \\ 28.10 & 2.094 & .501 & .06 & -.581 & .026 \\ 25.35 & 2.112 & .442 & .05 & -.588 & .027 \\ 28.60 & 2.131 & .392 & .03 & -.030 & .028 \\ 28.86 & 2.150 & .362 & .02 & -.022 & .030\end{array}$

$$
0_{2}^{+}: 0-7.65 \mathrm{MeV}
$$

$\begin{array}{llllll}2.87 & 0.218 & .097 & .033 & .240 & .196 \\ 3.99 & 0.297 & .148 & .026 & .136 & .060 \\ 4.94 & 0.368 & .175 & .018 & .112 & .040 \\ 5.66 & 0.419 & .223 & .022 & .161 & .040 \\ 6.62 & 0.494 & .388 & .032 & .052 & .028 \\ 7.33 & 0.548 & .497 & .046 & .057 & .037 \\ 8.29 & 0.619 & .832 & .068 & .272 & .026 \\ 9.01 & 0.674 & .976 & .060 & .331 & .019 \\ 9.96 & 0.745 & 1.32 & .097 & .375 & .014 \\ 10.68 & 0.800 & 1.59 & .11 & .409 & .011 \\ 11.63 & 0.870 & 1.66 & .12 & .461 & .011 \\ 12.35 & 0.926 & 1.66 & .12 & .451 & .010 \\ 13.31 & 0.998 & 1.59 & .11 & .468 & .010 \\ 14.02 & 1.050 & 1.65 & .12 & .484 & .010 \\ 14.98 & 1.122 & 1.27 & .090 & .472 & .010 \\ 15.69 & 1.173 & 1.08 & .083 & .431 & .019 \\ 16.64 & 1.242 & .930 & .073 & .433 & .019 \\ 17.36 & 1.299 & .869 & .074 & .42 & .016 \\ 18.31 & 1.368 & .601 & .047 & .323 & .020 \\ 19.03 & 1.424 & .512 & .038 & .295 & .016 \\ 19.98 & 1.494 & .307 & .024 & .118 & .021 \\ 20.69 & 1.547 & .205 & .024 & .064 & .024 \\ 21.64 & 1.617 & .125 & .012 & -.180 & .038 \\ 24.02 & 1.795 & .075 & .007 & -.470 & .040 \\ 24.97 & 1.863 & .028 & .005 & -.057 & .051 \\ 25.12 & 1.875 & .021 & .003 & -.613 & .170 \\ 26.07 & 1.942 & .023 & .003 & .500 & .057 \\ 27.34 & 2.037 & .034 & .007 & .869 & .060 \\ 28.28 & 2.098 & .040 & .008 & .858 & .019\end{array}$




$$
3: 0-9.64 \mathrm{MeV}
$$

$\begin{array}{llllll}5.66 & 0.419 & .071 & .013 & .459 & .091 \\ 6.62 & 0.494 & .134 & .018 & .613 & .055 \\ 7.33 & 0.548 & .149 & .029 & .564 & .037 \\ 8.29 & 0.619 & .327 & .036 & .613 & .041 \\ 9.01 & 0.674 & .291 & .029 & .555 & .035 \\ 9.96 & 0.745 & .685 & .056 & .698 & .021 \\ 10.68 & 0.800 & .923 & .069 & .708 & .017 \\ 11.63 & 0.870 & 1.34 & .099 & .713 & .015 \\ 12.35 & 0.926 & 1.49 & .108 & .730 & .014 \\ 13.31 & 0.998 & 1.78 & .13 & .736 & .014 \\ 14.02 & 1.050 & 2.15 & .15 & .743 & .014 \\ 14.98 & 1.122 & 2.28 & .16 & .739 & .014 \\ 15.69 & 1.173 & 2.34 & .16 & .709 & .017 \\ 16.64 & 1.242 & 2.55 & .20 & .684 & .016 \\ 17.36 & 1.299 & 2.82 & .21 & .689 & .014 \\ 18.31 & 1.368 & 3.01 & .23 & .655 & .013 \\ 19.03 & 1.424 & 2.71 & .19 & .660 & .013 \\ 19.98 & 1.494 & 2.82 & .13 & .595 & .010 \\ 20.69 & 1.547 & 2.53 & .18 & .582 & .012 \\ 21.64 & 1.617 & 2.46 & .18 & .524 & .012 \\ 24.02 & 1.795 & 1.87 & .14 & .373 & .010 \\ 24.97 & 1.863 & 1.44 & .11 & .295 & .012 \\ 25.12 & 1.875 & 1.26 & .090 & .270 & .009 \\ 26.07 & 1.942 & .792 & .057 & .231 & .012 \\ 27.34 & 2.037 & .740 & .056 & .182 & .018 \\ 28.29 & 2.098 & .443 & .035 & .060 & .024\end{array}$

$$
I_{1}^{-}: 0-10.84 \mathrm{MeV}
$$

$\begin{array}{rlllll}7.81 & 0.588 & .042 & .019 & .523 & .217 \\ 9.49 & 0.714 & .095 & .014 & .422 & .072 \\ 11.16 & 0.840 & .180 & .015 & .498 & .026 \\ 12.83 & 0.965 & .267 & .020 & .500 & .015 \\ 14.50 & 1.090 & .354 & .026 & .500 & .013 \\ 16.17 & 1.215 & .326 & .024 & .552 & .013 \\ 17.84 & 1.339 & .367 & .026 & .570 & .012 \\ 19.50 & 1.464 & .295 & .021 & .500 & .010 \\ 21.17 & 1.587 & .222 & .016 & .328 & .010\end{array}$




$$
4_{1}^{+}: 0-14.08 \mathrm{MeV}
$$

$\begin{array}{llllll}14.02 & 1.050 & .039 & .015 & .74,3 & .090 \\ 14.93 & 1.122 & .054 & .014 & .590 & .080 \\ 15.69 & 1.173 & .047 & .012 & .752 & .070 \\ 16.64 & 1.242 & .062 & .010 & .849 & .075 \\ 17.36 & 1.299 & .080 & .013 & .891 & .062 \\ 18.31 & 1.358 & .101 & .011 & .800 & .069 \\ 19.03 & 1.424 & .094 & .009 & .682 & .062 \\ 19.98 & 1.494 & .110 & .011 & .626 & .058 \\ 20.69 & 1.547 & .122 & .013 & .520 & .051 \\ 21.64 & 1.617 & .122 & .013 & .596 & .048 \\ 24.02 & 1.795 & .119 & .011 & .482 & .051 \\ 24.97 & 1.863 & .101 & .010 & .377 & .042 \\ 25.12 & 1.875 & .113 & .008 & .261 & .044 \\ 26.07 & 1.942 & .100 & .010 & .178 & .039 \\ 27.34 & 2.037 & .083 & .007 & -.069 & .062 \\ 28.28 & 2.098 & .058 & .007 & -.091 & .058\end{array}$

$$
1_{1}^{+}: 0-12.71 \mathrm{MeV}
$$

$\begin{array}{llllll}3.35 & 0.252 & .241 & .043 & -.383 & .092 \\ 3.83 & 0.290 & .265 & .033 & -.245 & .060 \\ 3.99 & 0.299 & .187 & .039 & -.244 & .118 \\ 4.46 & 0.337 & .248 & .027 & -.251 & .050 \\ 10 & 0.380 & .230 & .026 & -.219 & .051 \\ 5.50 & 0.413 & .271 & .027 & -.273 & .044 \\ 6.14 & 0.463 & .236 & .025 & -.313 & .047 \\ 6.78 & 0.510 & .224 & .024 & -.369 & .048 \\ 7.17 & 0.538 & .193 & .037 & -.410 & .102 . \\ 7.81 & 0.588 & .194 & .027 & -.361 & .070 \\ 8.45 & 0.638 & .181 & .025 & -.258 & .071 \\ 8.85 & 0.665 & .118 & .022 & -.410 & .102 \\ 9.49 & 0.714 & .165 & .020 & -.328 & .056 \\ 10.12 & 0.763 & .123 & .018 & -.449 & .070 \\ 10.52 & 0.791 & .163 & .017 & -.276 & .044 \\ 11.16 & 0.840 & .132 & .014 & -.353 & .045 \\ 11.79 & 0.888 & .109 & .012 & -.236 & .050 \\ 12.19 & 0.916 & .116 & .011 & -.323 & .036 \\ 12.83 & 0.965 & .083 & .009 & -.358 & .047 \\ 13.46 & 1.010 & .066 & .008 & -.340 & .056 \\ 13.86 & 1.042 & .073 & .009 & -.368 & .055 \\ 14.50 & 1.090 & .063 & .008 & -.226 & .059 \\ 15.13 & 1.138 & .042 & .007 & -.329 & .083 \\ 15.53 & 1.166 & .029 & .007 & -.261 & .096 \\ 16.17 & 1.215 & .039 & .005 & -.136 & .068 \\ 16.80 & 1.261 & .026 & .005 & -.266 & .096 \\ 17.20 & 1.290 & .032 & .004 & -.093 & .066 \\ 17.84 & 1.339 & .018 & .004 & -.357 & .131 \\ 18.47 & 1.387 & .021 & .004 & .096 & .085\end{array}$




$\begin{array}{llllll}18.37 & 1.415 & .016 & .003 & -.023 & .095 \\ 19.50 & 1.464 & .027 & .003 & .327 & .052 \\ 20.14 & 1.520 & .023 & .003 & .299 & .060 \\ 20.53 & 1.538 & .020 & .003 & .258 & .081 \\ 21.17 & 1.587 & .020 & .003 & .549 & .074 \\ 21.80 & 1.033 & .018 & .003 & .500 & .078 \\ 22.75 & 1.704 & .019 & .003 & .539 & .067 \\ 23.38 & 1.751 & .018 & .004 & .818 & .072 \\ 24.02 & 1.798 & .014 & .003 & .638 & .080 \\ 24.97 & 1.867 & .018 & .002 & .519 & .057 \\ 25.60 & 1.914 & .014 & .002 & .600 & .067 \\ 26.23 & 1.960 & .013 & .002 & .519 & .068 \\ 27.34 & 2.037 & .014 & .002 & .495 & .074 \\ 28.28 & 1.102 & .010 & .002 & .299 & .082\end{array}$

$$
1_{1}^{+} ; 1-15.11 \mathrm{MeV}
$$

$\begin{array}{llllll}3.35 & 0.252 & 1.95 & .153 & .001 & .021 \\ 3.33 & 0.290 & 1.82 & .141 & .045 & .020 \\ 3.99 & 0.299 & 1.29 & .105 & .026 & .025 \\ 4.46 & 0.337 & 1.42 & .108 & .007 & .018 \\ 5.10 & 0.380 & 1.07 & .083 & .023 & .021 \\ 5.50 & 0.413 & 1.13 & .086 & .028 & .019 \\ 6.14 & 0.463 & .893 & .070 & .050 & .022 \\ 6.78 & 0.510 & .681 & .055 & .069 & .026 \\ 1.17 & 0.538 & .557 & .056 & .031 & .043 \\ 7.81 & 0.588 & .496 & .047 & .026 & .040 \\ 8.45 & 0.638 & .361 & .038 & .091 & .047 \\ 8.85 & 0.665 & .320 & .029 & .042 & .065 \\ 9.49 & 0.714 & .358 & .032 & .102 & .033 \\ 10.12 & 0.763 & .285 & .027 & .154 & .037 \\ 10.52 & 0.791 & .255 & .023 & .065 & .033 \\ 11.16 & 0.840 & .188 & .018 & . .054 & .039 \\ 11.79 & 0.888 & .131 & .015 & .003 & .052 \\ 12.19 & 0.916 & .135 & .013 & . .001 & .037 \\ 12.83 & 0.965 & .097 & .010 & .004 & .048 \\ 13.46 & 1.010 & .055 & .008 & -.150 & .081 \\ 13.86 & 1.042 & .076 & .009 & .028 & .060 \\ 14.50 & 1.030 & .047 & .008 & -.055 & .094 \\ 15.13 & 1.138 & .032 & .007 & .150 & .115 \\ 15.53 & 1.165 & .024 & .005 & .223 & .117 \\ 16.17 & 1.215 & .024 & .005 & .040 & .117 \\ 16.89 & 1.261 & .019 & .005 & .127 & .138 \\ 17.20 & 1.290 & .013 & .004 & .034 & .175 \\ 17.84 & 1.339 & .017 & .004 & .114 & .124 \\ 18.47 & 1.387 & .013 & .004 & .048 & .156 \\ 18.87 & 1.415 & .011 & .003 & .237 & .134 \\ 19.50 & 1.464 & .013 & .003 & . .046 & .130 \\ 20.14 & 1.520 & .014 & .003 & . .165 & .117 \\ 20.53 & 1.538 & .017 & .003 & .210 & .098\end{array}$




$\begin{array}{llllll}21.17 & 1.587 & .012 & .003 & -.044 & .144 \\ 21.80 & 1.633 & .014 & .003 & -.138 & .113 \\ 22.75 & 1.704 & .011 & .003 & -.369 & .134 \\ 23.38 & 1.751 & .010 & .002 & -.155 & .122 \\ 24.02 & 1.798 & .009 & .002 & -.115 & .124 \\ 24.97 & 1.867 & .010 & .002 & -.253 & .108 \\ 25.60 & 1.914 & .008 & .002 & -.253 & .126 \\ 26.23 & 1.960 & .007 & .002 & -.480 & .136 \\ 27.34 & 2.037 & .007 & .002 & -.173 & .138 \\ 28.28 & 2.102 & .004 & .002 & -.451 & .231\end{array}$

$$
2_{1}^{+}: 1-15.11 \mathrm{MeV}
$$

$\begin{array}{llllrl}5.50 & 0.413 & .024 & .009 & .069 & .220 \\ 6.14 & 0.463 & .021 & .010 & -.097 & .284 \\ 6.78 & 0.510 & .048 & .011 & .190 & .139 \\ 7.17 & 0.538 & .030 & .024 & .359 & .452 \\ 7.81 & 0.588 & .038 & .014 & .290 & .207 \\ 8.45 & 0.638 & .061 & .016 & .195 & .150 \\ 8.85 & 0.665 & .040 & .023 & .534 & .271 \\ 9.49 & 0.714 & .092 & .014 & .058 & .093 \\ 10.12 & 0.763 & .096 & .0 .5 & .179 & .079 \\ 10.52 & 0.791 & .088 & .012 & .068 & .074 \\ 11.16 & 0.840 & .089 & .012 & -.119 & .070 \\ 11.79 & 0.888 & .093 & .012 & -.043 & .068 \\ 12.19 & 0.916 & .098 & .010 & -.033 & .049 \\ 12.83 & 0.955 & .098 & .010 & .008 & .046 \\ 13.46 & 1.010 & .095 & .011 & -.086 & .048 \\ 1.3 .36 & 1.042 & .100 & .010 & -.068 & .048 \\ 17.30 & 1.090 & .090 & .009 & .030 & .049 \\ 15.13 & 1.138 & .077 & .007 & -.091 & .057 \\ 15.53 & 1.166 & .067 & .007 & -.084 & .059 \\ 16.17 & 1.215 & .067 & .007 & -.084 & .048 \\ 16.80 & 1.261 & .060 & .006 & -.129 & .051 \\ 17.20 & 1.290 & .061 & .006 & -.085 & .044 \\ 17.84 & 1.339 & .053 & .005 & -.053 & .046 \\ 18.47 & 1.387 & .039 & .004 & -.190 & .060 \\ 18.87 & 1.415 & .042 & .004 & -.120 & .043 \\ 19.50 & 1.464 & .038 & .004 & -.135 & .045 \\ 20.14 & 1.510 & .032 & .003 & -.151 & .051 \\ 20.53 & 1.538 & .028 & .003 & -.102 & .063 \\ 21.17 & 1.587 & .024 & .003 & -.166 & .072 \\ 21.80 & 1.633 & .014 & .002 & . .167 & .108 \\ 22.75 & 1.704 & .013 & .002 & . .246 & .103 \\ 23.38 & 1.751 & .011 & .003 & -.126 & .110 \\ 24.02 & 1.798 & .006 & .002 & -.634 & .225 \\ 24.97 & 1.867 & .007 & .002 & -.107 & .129 \\ 25.60 & 1.914 & .005 & .001 & .056 & .165 \\ 26.23 & 1.960 & .004 & .001 & -.138 & .186 \\ 27.34 & 2.037 & .005 & .001 & . .019 & .156 \\ 28.28 & 2.102 & .004 & .001 & -.034 & .197\end{array}$




$$
2 \bar{j} ; 1-16.58 \mathrm{MeV}
$$

$\begin{array}{llllll}12.83 & 0.965 & .012 & .005 & .554 & .179 \\ 14.50 & 1.090 & .023 & .005 & .372 & .102 \\ 16.17 & 1.215 & .023 & .004 & .404 & .078 \\ 17.84 & 1.339 & .031 & .004 & .634 & .049 \\ 19.50 & 1.464 & .025 & .003 & .534 & .039 \\ 21.17 & 1.587 & .027 & .003 & .420 & .040 \\ 23.38 & 1.751 & .019 & .002 & .370 & .037 \\ 25.60 & 1.914 & .012 & .001 & .252 & .045 \\ 27.81 & 2.073 & .004 & .001 & .137 & .162\end{array}$

$$
2_{2}^{-} ; 0-13.35 \mathrm{MeV}
$$

$\begin{array}{llllll}19.50 & 1.464 & .009 & .003 & -.249 & .156 \\ 21.17 & 1.587 & .014 & .003 & .045 & .090 \\ 23.38 & 1.751 & .011 & .002 & .392 & .084 \\ 25.60 & 1.914 & .010 & .002 & .167 & .074 \\ 27.82 & 2.075 & .009 & .002 & .242 & .111\end{array}$

\begin{tabular}{|c|c|c|c|c|c|}
\hline $\begin{array}{l}4.46 \\
6.14 \\
7.81 \\
9.49 \\
1.16 \\
2.83 \\
4.50 \\
6.17 \\
7.84 \\
9.50 \\
1.17 \\
3.38 \\
5.60 \\
7.82\end{array}$ & $\begin{array}{l}0.337 \\
0.463 \\
0.588 \\
0.714 \\
0.840 \\
0.965 \\
1.090 \\
1.215 \\
1.339 \\
1.464 \\
1.587 \\
1.751 \\
1.914 \\
2.075\end{array}$ & $\begin{array}{l}.354 \\
.371 \\
.378 \\
.464 \\
.463 \\
.380 \\
.288 \\
.166 \\
.106 \\
.055 \\
.034 \\
.024 \\
.024 \\
.026\end{array}$ & $\begin{array}{l}.040 \\
.031 \\
.039 \\
.040 \\
.035 \\
.028 \\
.022 \\
.013 \\
.008 \\
.005 \\
.003 \\
.002 \\
.002 \\
.002\end{array}$ & $\begin{array}{r}-.251 \\
-.287 \\
-.164 \\
-.165 \\
-.159 \\
-.036 \\
-.106 \\
.040 \\
.155 \\
.337 \\
.170 \\
.204 \\
.145 \\
.062\end{array}$ & $\begin{array}{l}.053 \\
.027 \\
.047 \\
.031 \\
.017 \\
.013 \\
.016 \\
.018 \\
.020 \\
.023 \\
.037 \\
.034 \\
.029 \\
.037\end{array}$ \\
\hline & & & -19.28 & $\mathrm{MeV}$ & \\
\hline $\begin{array}{r}.17 \\
.84\end{array}$ & $\begin{array}{l}1.215 \\
1.339 \\
1.464 \\
1.587 \\
1.715 \\
1.914 \\
2.075\end{array}$ & $\begin{array}{l}.017 \\
.047 \\
.056 \\
.048 \\
.038 \\
.037 \\
.032\end{array}$ & $\begin{array}{l}.026 \\
.020 \\
.006 \\
.005 \\
.003 \\
.003 \\
.003\end{array}$ & $\begin{array}{r}-.430 \\
-.412 \\
-.177 \\
-.401 \\
.029 \\
.068 \\
-.002\end{array}$ & $\begin{array}{l}.797 \\
.221 \\
.038 \\
.012 \\
.037 \\
.031 \\
.047\end{array}$ \\
\hline
\end{tabular}

$$
(2 \overline{3} ; 0)-18.30 \mathrm{MeV}
$$




$$
(2-i 1)-19.40 \mathrm{MeV}
$$

$\begin{array}{rlllll}4.46 & 0.337 & .807 & .069 & .104 & .029 \\ 6.14 & 0.463 & .721 & .055 & .229 & .019 \\ 7.81 & 0.488 & .713 & .061 & .257 & .030 \\ 9.49 & 0.714 & .475 & .049 & .093 & .045 \\ 11.16 & 0.840 & .422 & .034 & .180 & .025 \\ 12.83 & 0.965 & .262 & .021 & .241 & .025 \\ 14.50 & 1.090 & .159 & .015 & .090 & .038 \\ 16.17 & 1.215 & .066 & .024 & -.015 & .209 \\ 17.84 & 1.339 & .027 & .018 & -.014 & .395\end{array}$

$$
\left(4_{1}^{-} ; 1\right)-19.65 \mathrm{MeV}
$$

$\begin{array}{rlllll}9.49 & 0.714 & .081 & .030 & .125 & .221 \\ 1.1 .16 & 0.840 & .074 & .015 & .111 & .115 \\ 12.83 & 0.965 & .082 & .011 & .147 & .067 \\ 14.50 & 1.090 & .175 & .015 & .198 & .030 \\ 16.17 & 1.215 & .181 & .016 & .317 & .028 \\ 17.84 & 1.339 & .224 & .017 & .258 & .019 \\ 19.50 & 1.464 & .209 & .015 & .301 & .010 \\ 21.17 & 1.587 & .202 & .015 & .292 & .012 \\ 23.38 & 1.715 & .148 & .017 & .293 & .011 \\ 25.60 & 1.914 & .104 & .008 & .309 & .012 \\ 27.82 & 2.075 & .072 & .006 & .279 & .021\end{array}$

$$
\left(3{ }_{1}^{-} ; 1\right)-20.60 \mathrm{MeV}
$$

$\begin{array}{llllll}12.83 & 0.965 & .101 & .009 & .025 & .036 \\ 14.50 & 1.090 & .103 & .009 & .205 & .034 \\ 16.17 & 1.215 & .091 & .008 & .209 & .029 \\ 17.84 & 1.339 & .078 & .007 & .111 & .026 \\ 19.50 & 1.464 & .053 & .004 & .183 & .026 \\ 21.17 & 1.587 & .039 & .004 & .066 & .036 \\ 23.38 & 1.751 & .021 & .002 & .052 & .045 \\ 25.60 & 1.914 & .013 & .001 & -.132 & .057 \\ 27.82 & 2.075 & -.006 & .002 & .207 & .152\end{array}$


Experimental Angular Distributions

$$
{ }^{12} \mathrm{C}\left(\overrightarrow{\mathrm{p}}, \mathrm{p}^{\prime}\right){ }^{12} \mathrm{C}^{*}-597 \mathrm{MeV}
$$

$\hat{c}_{c \Omega} q\left(f m^{-1}\right) \quad \frac{d \sigma}{d \Omega}\left(\frac{m b}{s I}\right)=\Delta \quad A_{Y} \pm \Delta$

$$
0_{1}^{+}, 0-0.00 \mathrm{MeV}
$$

$\begin{array}{llllll}3.36 & 0.317 & 2259 . & 25 . & .166 & .011 \\ 3.62 & 0.344 & 2172 . & 24 . & .155 & .011 \\ 3.88 & 0.370 & 1965 . & 23 . & .181 & .011 \\ 4.14 & 0.392 & 1847 . & 22 . & .212 & .012 \\ 4.40 & 0.419 & 1730 . & 22 . & .193 & .012 \\ 4.66 & 0.442 & 1556 . & 21 . & .227 & .013 \\ 4.80 & 0.456 & 1487 . & 20 . & .269 & .016 \\ 5.06 & 0.480 & 1460 . & 18 . & .293 & .016 \\ 5.32 & 0.505 & 1350 . & 16 . & .296 & .016 \\ 5.58 & 0.530 & 1244 . & 15 . & .297 & .017 \\ 5.98 & 0.569 & 1091 . & 13 . & .298 & .011 \\ 6.24 & 0.591 & 1060 . & 13 . & .297 & .011 \\ 6.50 & 0.618 & 996 . & 13 . & .322 & .012 \\ 6.76 & 0.634 & 905 . & 12 . & .331 & .012 \\ 7.02 & 0.666 & 846 . & 12 . & .319 & .013 \\ 7.28 & 0.693 & 759 . & 12 . & .331 & .013 \\ 7.54 & 0.714 & 708 . & 11 . & .325 & .014 \\ 7.90 & 0.741 & 662 . & 10 . & .365 & .014 \\ 8.06 & 0.766 & 596 . & 10 . & .353 & .015 \\ 8.24 & 0.781 & 545 . & 6.4 & .394 & .012 \\ 8.50 & 0.805 & 503 . & 6.0 & .384 & .013 \\ 8.76 & 0.831 & 447 . & 5.6 & .403 & .014 \\ 9.02 & 0.858 & 405 . & 5.5 & .415 & .014 \\ 9.28 & 0.880 & 364 . & 5.2 & .415 & .015 \\ 9.54 & 0.906 & 321 . & 4.9 & .408 & .016 \\ 9.80 & 0.928 & 286 . & 4.6 & .380 & .017 \\ 10.06 & 0.954 & 245 . & 4.2 & .401 & .018 \\ 10.32 & 0.979 & 214 . & 4.0 & .390 & .020 \\ 10.51 & 0.997 & 199 . & 2.5 & .369 & .013 \\ 10.76 & 1.019 & 173 . & 2.3 & .360 & .014 \\ 11.02 & 1.045 & 153 . & 2.2 & .370 & .015 \\ 11.29 & 1.071 & 126 . & 2.0 & .333 & .016 \\ 11.54 & 1.094 & 110 . & 1.8 & .347 & .017 \\ 11.80 & 1.117 & 95.4 & 1.7 & .334 & .019 \\ 12.06 & 1.142 & 77.3 & 1.5 & .326 & .021 \\ 12.32 & 1.168 & 61.56 & 1.4 & .348 & .022 \\ 12.58 & 1.190 & 55.1 & 1.3 & .301 & .025 \\ 12.76 & 1.210 & 47.6 & .51 & .271 & .012 \\ 13.02 & 1.233 & 38.6 & .48 & .251 & .014 \\ 13.28 & 1.258 & 31.2 & .38 & .219 & .015\end{array}$




$\begin{array}{llllll}13.54 & 1.285 & 25.0 & .38 & .207 & .017 \\ 13.80 & 1.307 & 19.4 & .33 & .182 & .020 \\ 14.06 & 1.330 & 15.5 & .30 & .125 & .022 \\ 14.32 & 1.355 & 11.4 & .26 & .120 & .026 \\ 14.58 & 1.381 & 8.48 & .22 & .019 & .030 \\ 14.83 & 1.402 & 5.69 & .18 & -.054 & .031 \\ 15.02 & 1.423 & 4.45 & .15 & -.192 & .041 \\ 15.28 & 1.445 & 3.21 & .13 & -.244 & .048 \\ 15.54 & 1.471 & 2.28 & .11 & -.384 & .056 \\ 15.80 & 1.495 & 1.38 & .08 & -.529 & .069 \\ 16.06 & 1.519 & .951 & .069 & -.515 & .084 \\ 16.31 & 1.544 & .720 & .060 & -.285 & .101 \\ 16.57 & 1.557 & .686 & .064 & .125 & .101 \\ 16.83 & 1.593 & .660 & .064 & .454 & .090 \\ 17.09 & 1.615 & .934 & .081 & .775 & .057 \\ 17.28 & 1.635 & 1.06 & .026 & .827 & .020 \\ 17.54 & 1.659 & 1.34 & .030 & .893 & .016 \\ 17.79 & 1.683 & 1.59 & .034 & .908 & .014 \\ 18.05 & 1.705 & 1.94 & .039 & .881 & .014 \\ 18.31 & 1.731 & 2.18 & .039 & .866 & .013 \\ 18.57 & 1.756 & 2.53 & .043 & .829 & .013 \\ 18.83 & 1.779 & 2.63 & .043 & .813 & .013 \\ 19.08 & 1.800 & 2.88 & .043 & .754 & .013 \\ 19.34 & .1 .326 & 2.70 & .043 & .778 & .013\end{array}$

$$
2_{1}^{+} ; 0-4.44 \mathrm{MeV}
$$

$\begin{array}{llllll}4.14 & 0.392 & 9.16 & .96 & .255 & .067 \\ 4.40 & 0.419 & 8.68 & .92 & .297 & .068 \\ 4.66 & 0.442 & 9.28 & 1.0 & .292 & .065 \\ 4.80 & 0.456 & 10.5 & 1.1 & .368 & .060 \\ 5.06 & 0.480 & 10.7 & 1.0 & .235 & .062 \\ 5.32 & 0.505 & 11.7 & 1.1 & .333 & .054 \\ 5.58 & 0.530 & 11.9 & 1.2 & .329 & .034 \\ 5.98 & 0.569 & 12.2 & 1.2 & .387 & .035 \\ 6.24 & 0.591 & 12.4 & 1.0 & .381 & .038 \\ 6.50 & 0.618 & 12.5 & 1.4 & .345 & .042 \\ 6.76 & 0.634 & 12.9 & 1.5 & .422 & .050 \\ 7.02 & 0.666 & 13.1 & 1.4 & .437 & .052 \\ 7.28 & 0.693 & 14.4 & 1.4 & .450 & .053 \\ 7.54 & 0.714 & 15.7 & 1.6 & .442 & .042 \\ 7.80 & 0.741 & 17.1 & 1.7 & .452 & .038 \\ 8.06 & 0.766 & 19.2 & 1.8 & .450 & .076 \\ 8.24 & 0.781 & 20.6 & 1.2 & .492 & .061 \\ 8.50 & 0.805 & 20.7 & 1.2 & .487 & .061 \\ 8.76 & 0.831 & 20.8 & 1.2 & .505 & .060 \\ 9.02 & 0.858 & 23.4 & 1.3 & .512 & .052 \\ 9.28 & 0.880 & 22.1 & 1.3 & .477 & .059 \\ 9.54 & 0.906 & 23.3 & 1.3 & .414 & .059 \\ 9.80 & 0.928 & 24.5 & 1.4 & .519 & .052 \\ 10.06 & 0.954 & 26.0 & 1.4 & .510 & .054\end{array}$




$\begin{array}{ll}10.32 & 0.979 \\ 10.51 & 0.997 \\ 10.76 & 1.019 \\ 11.02 & 1.045 \\ 11.28 & 1.071 \\ 11.54 & 1.094 \\ 11.80 & 1.117 \\ 12.06 & 1.142 \\ 12.32 & 1.168 \\ 12.58 & 1.190 \\ 12.76 & 1.210 \\ 13.02 & 1.233 \\ 13.28 & 1.258 \\ 13.54 & 1.285 \\ 13.80 & 1.307 \\ 14.06 & 1.330 \\ 14.32 & 1.355 \\ 14.58 & 1.381 \\ 14.83 & 1.402 \\ 15.02 & 1.423 \\ 15.28 & 1.445 \\ 15.54 & 1.471 \\ 15.80 & 1.495 \\ 16.06 & 1.519 \\ 16.31 & 1.544 \\ 16.57 & 1.567 \\ 16.83 & 1.593 \\ 17.09 & 1.615 \\ 17.28 & 1.635 \\ 17.54 & 1.659 \\ 17.79 & 1.683 \\ 18.05 & 1.705 \\ 18.31 & 1.731 \\ 18.57 & 1.756 \\ 18.83 & 1.779 \\ 19.08 & 1.800 \\ 19.34 & 1.826 \\ & \end{array}$

$\begin{array}{llll}26.3 & 1.4 & .532 & .053 \\ 28.6 & .94 & .484 & .032 \\ 28.5 & .94 & .486 & .032 \\ 27.4 & .94 & .533 & .032 \\ 27.9 & .94 & .517 & .032 \\ 27.5 & .94 & .538 & .031 \\ 27.0 & .94 & .491 & .033 \\ 28.0 & .94 & .498 & .032 \\ 27.8 & .94 & .493 & .032 \\ 26.0 & .90 & .497 & .033 \\ 25.2 & .39 & .506 & .016 \\ 24.8 & .39 & .496 & .016 \\ 25.4 & .40 & .503 & .015 \\ 23.2 & .37 & .461 & .017 \\ 22.5 & .36 & .477 & .017 \\ 21.7 & .36 & .469 & .017 \\ 20.5 & .35 & .473 & .017 \\ 20.1 & .35 & .428 & .018 \\ 18.5 & .33 & .438 & .018 \\ 17.6 & .33 & .447 & .018 \\ 16.7 & .32 & .434 & .018 \\ 15.8 & .31 & .427 & .019 \\ 14.3 & .27 & .393 & .020 \\ 13.8 & .29 & .418 & .020 \\ 12.8 & .28 & .375 & .021 \\ 11.6 & .27 & .415 & .022 \\ 10.5 & .25 & .392 & .023 \\ 9.80 & .24 & .352 & .025 \\ 9.05 & .08 & .349 & .009 \\ 8.19 & .07 & .331 & .010 \\ 7.50 & .07 & .317 & .010 \\ 6.57 & .07 & .289 & .011 \\ 5.96 & .06 & .283 & .012 \\ 5.37 & .06 & .256 & .012 \\ 4.70 & .06 & .242 & .013 \\ 4.19 & .05 & .203 & .014 \\ 3.69 & .05 & .184 & .015\end{array}$

$\mathrm{O}_{2}^{+} ; 0-7.65 \mathrm{MeV}$

$\begin{array}{rlllll}2.91 & 0.279 & .155 & .063 & -.238 & .210 \\ 3.88 & 0.371 & .301 & .056 & -.172 & .072 \\ 4.61 & 0.443 & .458 & .065 & -.152 & .035 \\ 5.58 & 0.530 & 1.26 & .168 & .066 & .018 \\ 6.31 & 0.598 & 1.70 & .26 & .067 & .049 \\ 7.28 & 0.689 & 2.55 & .37 & .230 & .035 \\ 8.57 & 0.814 & 3.38 & .46 & .235 & .025 \\ 9.54 & 0.906 & 3.56 & .48 & .330 & .023 \\ 10.83 & 1.028 & 3.55 & .46 & .337 & .006 \\ 11.80 & 1.116 & 2.97 & .38 & .333 & .006 \\ 13.09 & 1.241 & 2.42 & .32 & .334 & .013\end{array}$




$\begin{array}{ll}14.06 & 1.330 \\ 15: 35 & 1.448 \\ 16.31 & 1.540 \\ 17.60 & 1.666 \\ 13.57 & 1.752\end{array}$

1.51

.891

.380

.157

.042

$$
3: 0-9.64 \mathrm{MeV}
$$

$\begin{array}{ll}4.61 & 0.443 \\ 5.58 & 0.530 \\ 6.31 & 0.598 \\ 7.28 & 0.689 \\ 8.57 & 0.814 \\ 9.54 & 0.906 \\ 10.83 & 1.028 \\ 11.80 & 1.116 \\ 13.09 & 1.241 \\ 14.06 & 1.330 \\ 15.35 & 1.448 \\ 16.31 & 1.540 \\ 17.60 & 1.666 \\ 18.57 & 1.752\end{array}$

$\begin{array}{rr}6.79 & 0.644 \\ 9.05 & 0.858 \\ 11.31 & 1.071 \\ 13.57 & 1.285\end{array}$

.238
.570
.678
1.61
2.49
3.85
5.17
6.16
7.14
8.49
6.62
5.61
4.58
3.38

.043
.080
.097
.21
.32
.50
.67
.80
.93
.1
.86
.73
.59
.44

208

.297

.347

.448

.486

.512

.529

.532

.541

.516

.488

.463

.418

.374

.057
.031
.033
.015
.011
.009
.007
.007
.009
.009
.009
.009
.007
.008

$1_{1}: 0-10.84 \mathrm{MeV}$

$\begin{array}{llll}.113 & .028 & .314 & .113 \\ .399 & .055 & .434 & .026 \\ .714 & .093 & .407 & .010 \\ .729 & .095 & .372 & .008\end{array}$

$\begin{array}{rlllll}4.61 & 0.443 & .087 & .032 & .016 & .149 \\ 5.58 & 0.530 & .096 & .033 & .340 & .126 \\ 6.79 & 0.644 & .107 & .022 & .425 & .091 \\ 10.83 & 1.028 & .091 & .022 & .322 & .100 \\ 11.80 & 1.116 & .109 & .021 & .352 & .098 \\ 13.09 & 1.241 & .180 & .026 & .701 & .106 \\ 14.06 & 1.330 & .195 & .028 & .557 & .087 \\ 15.35 & 1.448 & .221 & .031 & .419 & .079 \\ 16.31 & 1.540 & .216 & .028 & .465 & .078 \\ 17.60 & 1.666 & .259 & .021 & .324 & .059 \\ 18.57 & 1.752 & .134 & .016 & .320 & .060\end{array}$




$$
I_{1}^{+} ; 0-12.71 \mathrm{MeV}
$$

$\begin{array}{rlllll}3.88 & 0.370 & .384 & .068 & -.391 & .058 \\ 4.68 & 0.448 & . .28 & .049 & -.464 & .045 \\ 5.58 & 0.530 & .281 & .044 & -.398 & .046 \\ 6.27 & 0.597 & .225 & .046 & -.366 & .808 \\ 7.28 & 0.693 & .204 & .033 & -.311 & .049 \\ 8.56 & 0.812 & .142 & .028 & -.315 & .076 \\ 9.54 & 0.906 & .094 & .018 & -.163 & .075 \\ 10.81 & 1.026 & .079 & .012 & -.008 & .058 \\ 11.80 & 1.117 & .048 & .009 & .009 & .077 \\ 13.09 & 1.239 & .032 & .006 & .253 & .083 \\ 14.06 & 1.330 & .024 & .006 & .297 & .102 \\ 15.34 & 1.452 & .018 & .004 & .284 & .094 \\ 16.31 & 1.544 & .016 & .004 & .284 & .098 \\ 17.61 & 1.665 & .015 & .003 & .430 & .087 \\ 18.57 & 1.756 & .014 & .003 & .549 & .082\end{array}$

$$
I_{1}^{+} ; 1-15.11 \mathrm{MeV}
$$

$\begin{array}{rr}3.88 & 0.370 \\ 4.68 & 0.448 \\ 5.58 & 0.530 \\ 6.27 & 0.597 \\ 7.28 & 0.693 \\ 8.56 & 0.812 \\ 9.54 & 0.906 \\ 10.81 & 1.026 \\ 11.80 & 1.117 \\ 13.09 & 1.239 \\ 14.06 & 1.330 \\ 15.34 & 1.452 \\ 16.31 & 1.544 \\ 17.61 & 1.665 \\ 18.57 & 1.756\end{array}$

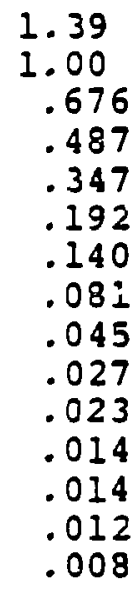

$$
.189
$$

.104

.028

$\begin{array}{ll}.00 & .134 \\ .676 & .093 \\ .487 & .071 \\ .347 & .051 \\ .192 & .032 \\ .140 & .026 \\ .081 & .015 \\ .045 & .011 \\ .027 & .008 \\ .023 & .006 \\ .014 & .004 \\ .014 & .003 \\ .012 & .003 \\ .008 & .003\end{array}$

.046

.021

$.033 \quad .027$

$-.006 \quad .039$
.046

$.046 \quad .039$

$.015 \quad .061$

$-.106$

.073

$-.014$

.112

$$
-.009
$$

.151

$-.242$

.149

$-.081$

.149

$-.134$

.126

.045

.120

$-.220$

.126

$$
2_{1}^{+}: 1-16.11 \mathrm{MeV}
$$

$\begin{array}{rr}4.68 & 0.448 \\ 5.58 & 0.530 \\ 6.27 & 0.597 \\ 7.28 & 0.693 \\ 8.56 & 0.812 \\ 9.54 & 0.906 \\ 10.81 & 1.026 \\ 11.80 & 1.117 \\ 13.09 & 1.239\end{array}$

$\begin{array}{ll}.044 & .015 \\ .056 & .016 \\ .053 & .030 \\ .061 & .021 \\ .087 & .021 \\ .091 & .019 \\ .096 & .016 \\ .082 & .014 \\ .076 & .012\end{array}$

$\begin{array}{rr}-.292 & .179 \\ .055 & .150 \\ -.272 & .299 \\ -.312 & .166 \\ .215 & .111 \\ .095 & .094 \\ -.059 & .060 \\ .133 & .058 \\ -.014 & .047\end{array}$




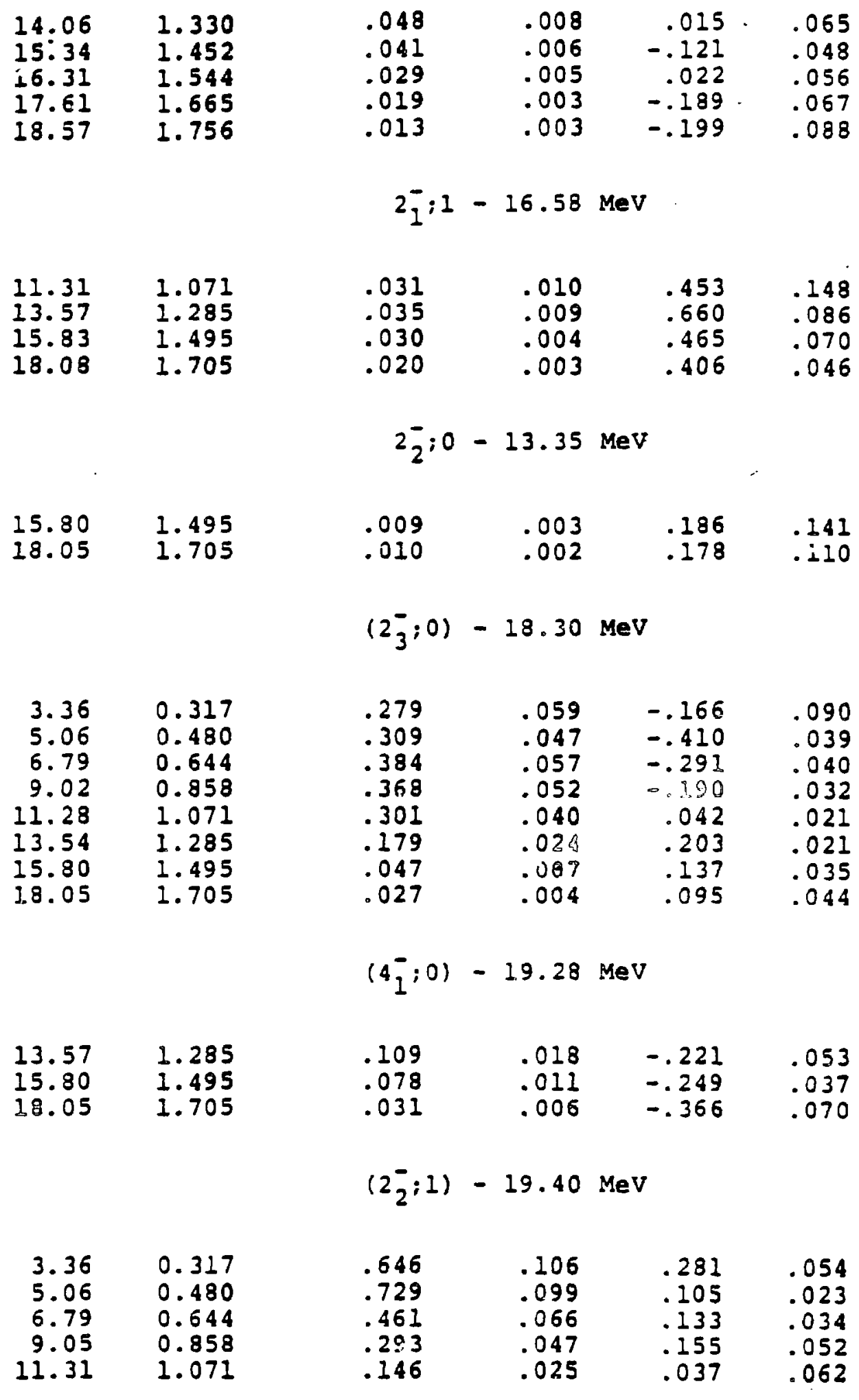


$\left(4_{i}^{-} ; 1\right)-19.65 \mathrm{MeV}$

$\begin{array}{rrrrrr}9.05 & 0.858 & .052 & .025 & -.081 & .262 \\ 11.31 & 1.071 & .152 & .034 & .235 & .049 \\ 13.57 & 1.285 & .287 & .038 & .212 & .017 \\ 15.00 & 1.495 & .225 & .030 & .224 & .012 \\ 18.05 & .1 .705 & .153 & .020 & .216 & .012 \\ & & & & & \end{array}$

$\begin{array}{rrrrrr}9.02 & 0.858 & .084 & .017 & .070 & .094 \\ 11.28 & 1.071 & .086 & .014 & -.004 & .056 \\ 13.54 & 1.285 & .099 & .014 & .138 & .035 \\ 15.80 & 1.495 & .028 & .042 & .072 & .042 \\ 18.05 & 1.705 & .036 & .052 & .0131 & .035\end{array}$


Experimental Angular Distributions

$$
{ }^{12} \mathrm{C}\left(\overrightarrow{\mathrm{p}}, \mathrm{p}^{1}\right)^{-12} \mathrm{C}^{*}-698 \mathrm{MeV}
$$

$\theta_{\mathrm{cm}} q\left(\mathrm{~m}^{-1}\right) \quad \frac{d \sigma}{d \Omega}\left(\frac{m b}{s r}\right) \pm \Delta \quad A_{Y} \pm \Delta$

$$
0_{1}^{+} ; 0-0.00 \mathrm{MeV}
$$

$\begin{array}{llllll}3.68 & 0.390 & 2623 . & 23 . & .189 & .011 \\ 3.94 & 0.419 & 2452 . & 22 . & .225 & .011 \\ 4.20 & 0.445 & 2317 . & 21 . & .223 & .012 \\ 4.47 & 0.467 & 2166 . & 21 . & .243 & .012 \\ 4.73 & 0.496 & 2015 . & 20 . & .240 & .012 \\ 4.99 & 0.521 & 1869 . & 17 . & .239 & .015 \\ 6.44 & 0.671 & 1117 . & 13 . & .262 & .015 \\ 6.70 & 0.696 & 1027 . & 12 . & .295 & .015 \\ 6.96 & 0.725 & 936 . & 12 . & .291 & .016 \\ 7.22 & 0.749 & 864 . & 11 . & .308 & .016 \\ 7.49 & 0.778 & 763 . & 10 . & .311 & .017 \\ 7.75 & 0.800 & 704 . & 10 . & .336 & .018 \\ 8.01 & 0.831 & 634 . & 9.5 & .335 & .019 \\ 9.47 & 0.978 & 294 . & 4.0 & .328 & .019 \\ 9.73 & 1.005 & 259 . & 4.0 & .321 & .018 \\ 9.99 & 1.032 & 219.0 & 3.5 & .341 & .019 \\ 10.26 & 1.061 & 186 . & 3.3 & .321 & .017 \\ 10.52 & 1.091 & 153 . & 3.0 & .332 & .016 \\ 10.78 & 1.113 & 131 . & 2.7 & .309 & .015 \\ 11.04 & 1.140 & 109 . & 2.5 & .290 & .016 \\ 11.30 & 1.168 & 89.1 & 2.3 & .284 & .016 \\ 11.56 & 1.195 & 74.4 & 2.1 & .281 & .014 \\ 12.89 & 1.330 & 18.2 & .23 & .180 & .016 \\ 13.15 & 1.357 & 13.6 & .20 & .130 & .019 \\ 13.41 & 1.382 & 9.79 & .17 & .076 & .022 \\ 13.67 & 1.412 & 7.00 & .14 & -.041 & .027 \\ 13.93 & 1.841 & 4.22 & .11 & -.033 & .034 \\ 14.19 & 1.464 & 2.83 & .09 & -.234 & .042 \\ 14.45 & 1.488 & 1.77 & .07 & . .214 & .053 \\ 14.71 & 1.518 & 1.19 & .06 & . .046 & .064 \\ 14.97 & 1.543 & 1.07 & .06 & .224 & .066 \\ 16.30 & 1.680 & 2.53 & .05 & .822 & .020 \\ 16.56 & 1.702 & 2.99 & .06 & .757 & .019 \\ 16.82 & 1.733 & 3.37 & .06 & .759 & .018 \\ 17.08 & 1.760 & 3.72 & .07 & .718 & .018 \\ 17.34 & 1.790 & 4.13 & .07 & .718 & .017 \\ 17.60 & 1.814 & 4.43 & .07 & .680 & .017 \\ 17.86 & 1.839 & 4.50 & .07 & .643 & .017 \\ 18.12 & 1.865 & 4.75 & .08 & .629 & .017 \\ 18.38 & 1.890 & 4.69 & .08 & .624 & .017\end{array}$




$$
2_{1}^{+} ; 0-4.44 \mathrm{MeV}
$$

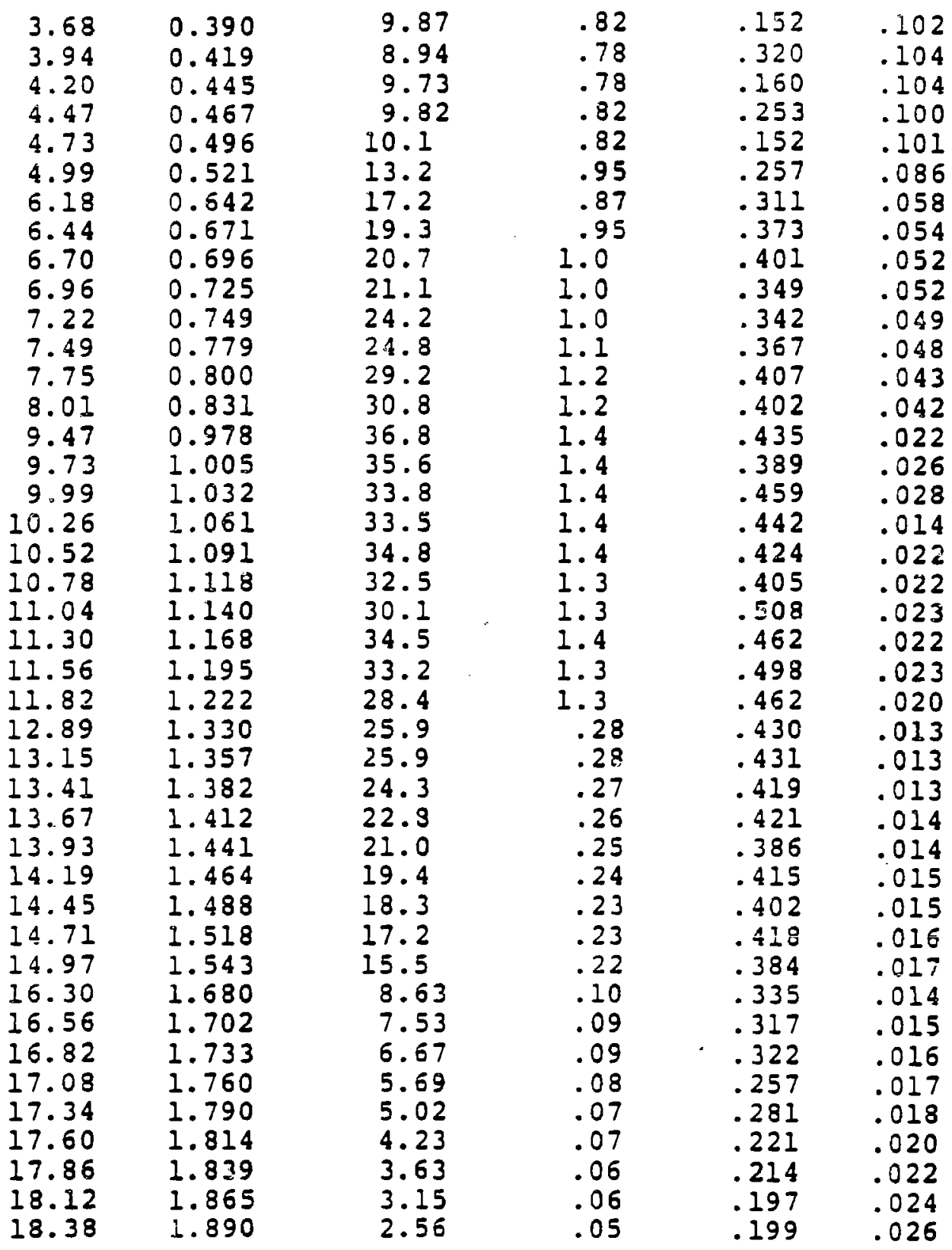




$$
0_{2}^{+} ; 0-7.65 \mathrm{MeV}
$$

$\begin{array}{rcccrr}3.42 & 0.355 & .243 & .045 & -.106 & .094 \\ 4.64 & 0.483 & .702 & .144 & -.058 & .024 \\ 5.62 & 0.586 & 1.82 & .37 & .107 & .013 \\ 6.21 & 0.645 & 1.98 & .40 & .147 & .020 \\ 7.19 & 0.747 & 3.56 & .72 & .223 & .012 \\ 9.77 & 1.006 & 4.60 & .93 & .299 & .014 \\ 10.74 & 1.117 & 3.67 & .74 & .310 & .015 \\ 13.18 & 1.358 & 1.74 & .34 & .295 & .015 \\ 14.15 & 1.463 & .764 & .16 & .247 & .022 \\ 16.59 & 1.704 & .089 & .02 & -.010 & .033 \\ 17.56 & 1.812 & .061 & .02 & .426 & .042 \\ & & 3-0.0 & & \end{array}$

$\begin{array}{rr}3.42 & 0.355 \\ 4.64 & 0.483 \\ 5.62 & 0.586 \\ 6.21 & 0.645 \\ 7.19 & 0.747 \\ 9.77 & 1.006 \\ 10.74 & 1.117 \\ 13.18 & 1.358 \\ 14.15 & 1.463 \\ 16.59 & 1.704 \\ 17.56 & 1.812\end{array}$

$\begin{array}{llll}.221 & .042 & .210 & .097 \\ .411 & .087 & .317 & .038 \\ 1.02 & .21 & .314 & .020 \\ 1.15 & .24 & .398 & .035 \\ 2.83 & .57 & .417 & .015 \\ 7.02 & 1.42 & .416 & .014 \\ 8.26 & 1.67 & .455 & .014 \\ 9.28 & 1.87 & .464 & .012 \\ 7.89 & 1.59 & .437 & .011 \\ 4.99 & 1.00 & .388 & .008 \\ 3.18 & .64 & .333 & .008\end{array}$

$1_{1}^{-} ; 0-10.84 \mathrm{MeV}$

$\begin{array}{llllll}5.13 & 0.533 & .049 & .016 & .116 & .160 \\ 6.70 & 0.696 & .197 & .054 & .108 & .108\end{array}$

$$
4_{1}^{+}: 0-14.08 \mathrm{MeV}
$$

$\begin{array}{rlllll}5.13 & 0.533 & .091 & .016 & .145 & .086 \\ 9.77 & 1.006 & .093 & .038 & .467 & .100 \\ 10.74 & 1.117 & .135 & .033 & .688 & .090 \\ 13.18 & 1.358 & .270 & .065 & .600 & .088 \\ 14.15 & 1.463 & .308 & .068 & .447 & .080 \\ 16.59 & 1.704 & .296 & .062 & .364 & .078 \\ 17.56 & 1.812 & .201 & .045 & .301 & .076\end{array}$




$$
1_{1}^{+} ; 0-12.71 \mathrm{MeV}
$$

$\begin{array}{rr}2.77 & 0.297 \\ 3.42 & 0.362 \\ 4.07 & 0.424 \\ 4.48 & 0.467 \\ 5.13 & 0.536 \\ 5.78 & 0.612 \\ 6.05 & 0.638 \\ 6.70 & 0.696 \\ 7.35 & 0.763 \\ 9.60 & 0.992 \\ 10.26 & 1.061 \\ 10.91 & 1.129 \\ 13.02 & 1.342 \\ 13.67 & 1.412 \\ 14.32 & 1.473 \\ 16.43 & 1.691 \\ 17.07 & 1.760 \\ 17.72 & 1.828\end{array}$

$\begin{array}{llll}.273 & .097 & -.185 & .173 \\ .330 & .086 & -.294 & .089 \\ .339 & .083 & -.052 & .073 \\ .290 & .066 & -.031 & .051 \\ .241 & .058 & -.410 & .055 \\ .236 & .055 & -.057 & .055 \\ .242 & .085 & -.246 & .168 \\ .206 & .051 & -.203 & .079 \\ .235 & .038 & -.120 & .112 \\ .068 & .018 & -.055 & .097 \\ .044 & .012 & .259 & .109 \\ .040 & .012 & .181 & .126 \\ .018 & .007 & .260 & .201 \\ .015 & .007 & .266 & .256 \\ .015 & .006 & .242 & .212 \\ .017 & .005 & .550 & .094 \\ .012 & .004 & .521 & .125 \\ .011 & .004 & .591 & .117\end{array}$

$$
1_{1}^{+} ; 1-15.11 \mathrm{MeV}
$$

\begin{tabular}{|c|c|c|c|c|c|}
\hline $\begin{array}{r}2.77 \\
3.42 \\
4.07 \\
4.48 \\
5.13 \\
5.78 \\
6.05 \\
6.70 \\
7.35 \\
9.60 \\
10.26 \\
10.91 \\
13.02 \\
13.67 \\
14.32 \\
16.43 \\
17.07 \\
17.72\end{array}$ & $\begin{array}{l}0.297 \\
0.362 \\
0.424 \\
3.467 \\
0.536 \\
0.612 \\
0.638 \\
0.696 \\
0.763 \\
0.992 \\
1.061 \\
1.129 \\
1.342 \\
1.412 \\
1.478 \\
1.691 \\
1.760 \\
1.828\end{array}$ & $\begin{array}{r}.930 \\
1.20 \\
.935 \\
.718 \\
.550 \\
.409 \\
.324 \\
.322 \\
.202 \\
.100 \\
.075 \\
.051 \\
.024 \\
.021 \\
.018 \\
.013 \\
.010 \\
.009\end{array}$ & $\begin{array}{l}.214 \\
.261 \\
.210 \\
.143 \\
.119 \\
.090 \\
.101 \\
.076 \\
.055 \\
.025 \\
.020 \\
.015 \\
.009 \\
.008 \\
.007 \\
.004 \\
.003 \\
.003\end{array}$ & $\begin{array}{r}-.049 \\
-.016 \\
-.025 \\
-.054 \\
.019 \\
-.102 \\
.007 \\
-.237 \\
.122 \\
.046 \\
.152 \\
-.014 \\
.323 \\
.236 \\
-.292 \\
-.172 \\
-.245 \\
-.042\end{array}$ & $\begin{array}{l}.060 \\
.037 \\
.041 \\
.027 \\
.034 \\
.043 \\
.143 \\
.075 \\
.104 \\
.083 \\
.097 \\
.136 \\
.167 \\
.177 \\
.217 \\
.116 \\
.158 \\
.152\end{array}$ \\
\hline \multicolumn{6}{|c|}{$2_{1}^{+}: 1-16.11 \mathrm{MeV}$} \\
\hline $\begin{array}{r}5.13 \\
9.60 \\
10.26\end{array}$ & $\begin{array}{l}0.536 \\
0.992 \\
1.061\end{array}$ & $\begin{array}{l}.038 \\
.100 \\
.084\end{array}$ & $\begin{array}{l}.020 \\
.025 \\
.021\end{array}$ & $\begin{array}{r}-.001 \\
.223 \\
.150\end{array}$ & $\begin{array}{l}.288 \\
.075 \\
.080\end{array}$ \\
\hline
\end{tabular}




$\begin{array}{llllrl}10.91 & 1.129 & .055 & .017 & .161 & .120 \\ 13: 02 & 1.342 & .057 & .014 & -.016 & .073 \\ 13.67 & 1.412 & .039 & .010 & .066 & .092 \\ 14.32 & 1.478 & .034 & .009 & -.073 & .098 \\ 16.43 & 1.691 & .016 & .004 & -.294 & .098 \\ 17.07 & 1.760 & .013 & .004 & -.403 & .111 \\ 17.72 & 1.828 & .009 & .003 & -.406 & .146\end{array}$

$$
2 ; 1 I-16.58 \mathrm{MeV}
$$

$$
\begin{aligned}
& \begin{array}{llllll}
10.26 & 1.061 & .033 & .010 & .424 & .127 \\
13.67 & 1.412 & .037 & .009 & .442 & .064 \\
17.07 & 1.760 & .020 & .004 & .483 & .047
\end{array} \\
& 2_{2}^{-} ; 0-13.35 \mathrm{MeV} \\
& 17.07 \quad 1.760 \\
& \begin{array}{llll}
.014 \quad .004 \quad .389 \quad .099
\end{array} \\
& (2-; 0)-18.30 \mathrm{MeV}
\end{aligned}
$$

$\begin{array}{rr}3.42 & 0.355 \\ 5.13 & 0.533 \\ 6.70 & 0.696 \\ 10.26 & 1.061 \\ 13.67 & 1.412 \\ 17.07 & 1.760\end{array}$

.309

.404

.546

.319

.082

.030

$$
.089
$$$$
-.481
$$

$$
\begin{array}{r}
.088 \\
.127
\end{array}
$$$$
.067
$$$$
-.475
$$$$
.044
$$$$
.085
$$$$
-.075
$$$$
\begin{aligned}
& .018 \\
& .007
\end{aligned}
$$$$
.186
$$

$$
\left(4_{1}^{-} ; 0\right)-19.28 \mathrm{MeV}
$$

$\begin{array}{ll}13.67 & 1.412 \\ 17.07 & 1.760\end{array}$

$$
.061
$$

.074

$$
.016
$$

$-.487$

$-.035$

.086

.025

$$
\left(2_{1}^{-} ; 1\right)-19.40 \mathrm{MeV}
$$

\footnotetext{
3.420 .355

5.13

0.533

6.70

0.696

10.26

1.061
}

.607

.139

$\begin{array}{rr}.042 & .058 \\ -.024 & .023 \\ .041 & .091 \\ -.0197 & .045\end{array}$

.869

.484

.151

.125

.035 
$\left(4_{1}^{-} ; 1\right)-19.65 \mathrm{MeV}$

$\begin{array}{rlllll}6.70 & 0.696 & .047 & .027 & .310 & .310 \\ 10.26 & 1.061 & .138 & .032 & .172 & .060 \\ 13.67 & 1.412 & .232 & .049 & .181 & .018 \\ 17.07 & 1.760 & .174 & .036 & .209 & .011 \\ & & & & & \\ & & (3-; 1) & -20.60 \mathrm{MeV} & & \\ 10.26 & 1.061 & .071 & .016 & -.083 & .062 \\ 13.67 & 1.412 & .060 & .013 & .023 & .045 \\ 17.07 & 1.760 & .019 & .004 & .062 & .057\end{array}$

